\title{
ON THE GENERIC PART OF THE COHOMOLOGY OF COMPACT UNITARY SHIMURA VARIETIES
}

\author{
ANA CARAIANI AND PETER SCHOLZE
}

\begin{abstract}
The goal of this paper is to show that the cohomology of compact unitary Shimura varieties is concentrated in the middle degree and torsion-free, after localizing at a maximal ideal of the Hecke algebra satisfying a suitable genericity assumption. Along the way, we establish various foundational results on the geometry of the Hodge-Tate period map. In particular, we compare the fibres of the Hodge-Tate period map with Igusa varieties.
\end{abstract}

\section{Contents}

1. Introduction

2. Refining the Hodge-Tate period map

2.1. Recollections on the Hodge-Tate period map 10

2.2. The $p$-adic-de Rham comparison isomorphism 13

2.3. Hodge cycles and torsors 16

3. The Newton stratification on the flag variety 23

3.1. Background on isocrystals with $G$-structure 23

3.2. The Fargues-Fontaine curve 25

3.3. The relative Fargues-Fontaine curve 29

3.4. The mixed characteristic affine Grassmannian 30

3.5. Vector bundles over $\mathcal{X}$ and the Newton stratification 33

4. The geometry of Newton strata and Igusa varieties 37

4.1. Preliminaries on $p$-divisible groups 37

4.2. Rapoport-Zink spaces of PEL type 42

4.3. A product formula 56

4.4. Étale cohomology 66

5. The cohomology of Igusa varieties 70

5.1. Setup 70

5.2. A stable trace formula 71

5.3. Base change and the twisted trace formula 75

5.4. The transfer at $p \quad 77$

5.5. Generic principal series $\quad 79$

5.6. Simple Shimura varieties 83

6. Torsion in the cohomology of unitary Shimura varieties 85

6.1. Perverse sheaves on the flag variety 85

6.2. A genericity assumption 89

6.3. Conclusion $\quad 90$

References

A.C. was partially supported by the NSF Postdoctoral Fellowship DMS-1204465 and NSF Grant DMS-1501064. 


\section{INTRODUCTION}

Let $G / \mathbb{Q}$ be a reductive group. The real group $G(\mathbb{R})$ acts on its associated symmetric domain $X=G(\mathbb{R}) / K_{\infty}$, where $K_{\infty} \subset G(\mathbb{R})$ is a maximal compact subgroup. For any congruence subgroup $\Gamma \subset G(\mathbb{Q})$, one can form the locally symmetric space

$$
X_{\Gamma}=\Gamma \backslash X
$$

We assume that $X_{\Gamma}$ is compact, and that $\Gamma$ is torsion-free. Then Matsushima's formula, Mat67, expresses the cohomology groups $H^{i}\left(X_{\Gamma}, \mathbb{C}\right)$ with complex coefficients in terms of automorphic forms $\pi$ on $G$, and the $\left(\mathfrak{g}, K_{\infty}\right)$-cohomology of their archimedean component $\pi_{\infty}$ 1] A computation of $\left(\mathfrak{g}, K_{\infty}\right)$-cohomology then shows that the part of cohomology to which tempered representations contribute is concentrated in the middle range $q_{0} \leq i \leq q_{0}+l_{0}$, cf. [BW80, Theorem III.5.1]; here $l_{0}=\operatorname{rk} G-\operatorname{rk} K_{\infty}$, and $q_{0}=\frac{1}{2}\left(\operatorname{dim} X-l_{0}\right)$.

In particular, if $l_{0}=0$, then tempered representations occur only in the middle degree $q_{0}$. This happens when the $X_{\Gamma}$ are complex algebraic varieties, e.g. when $G$ gives rise to a Shimura variety.

The motivating question of this paper is to establish a similar result for the cohomology groups $H^{i}\left(X_{\Gamma}, \mathbb{F}_{\ell}\right)$ with torsion coefficients. In this context, it is difficult to formulate the analogue of the temperedness condition, which is an analytic one. We learnt the following formulation from M. Emerton. Recall that for any system $\mathfrak{m}$ of Hecke eigenvalues appearing in $H^{i}\left(X_{\Gamma}, \mathbb{F}_{\ell}\right)$, one expects to have a mod $\ell$ Galois representation $\rho_{\mathfrak{m}}$ (with values in the Langlands dual group). One may then put the condition that $\rho_{\mathfrak{m}}$ is irreducible, and ask whether this implies that $q_{0} \leq i \leq q_{0}+l_{0}$. In particular, a result of this type for $G=G L_{n}$ (where $l_{0}>0$ ) is important for automorphy lifting theorems in the non-self dual case as in work of Calegari-Geraghty, CG, Conjecture B].

In the present paper, we deal with this question in the case where $X_{\Gamma}$ is a Shimura variety (so that $l_{0}=0$ ). More precisely, we will consider the case where $G$ is an anisotropic unitary similitude group of dimension $n$, for some CM field $F$ with totally real subfield $F^{+} \subset F$. We assume that $F$ contains an imaginary-quadratic field. Assume moreover that $G$ is associated with a division algebra over $F$, i.e., it is one of Kottwitz' simple Shimura varieties, Kot92a. 2 Our main theorem is the following.

Theorem 1.1. Let $\mathfrak{m}$ be a system of Hecke eigenvalues appearing in $H^{i}\left(X_{\Gamma}, \mathbb{F}_{\ell}\right)$. Then there is an associated Galois representation

$$
\rho_{\mathfrak{m}}: \operatorname{Gal}(\bar{F} / F) \rightarrow G L_{n}\left(\overline{\mathbb{F}}_{\ell}\right) .
$$

Assume that there is a rational prime $p$ such that $F$ is completely decomposed above $p$, and

$\rho_{\mathfrak{m}}$ is unramified and decomposed generic

at all places of $F$ above $p$. Then $i=q_{0}$ is the middle degree.

Remark 1.2. The first part of the theorem can be deduced from Sch15b, but we give a different proof in this paper. We will make use of the Hodge-Tate period

\footnotetext{
${ }^{1}$ In the non-compact case, this is still true, and a theorem of Franke, Fra98.

${ }^{2}$ We also allow the complementary case where $G$ is quasisplit at all finite places, under a small extra assumption (cf. Section [5.1), so that our main result also covers cases where nontrivial endoscopy occurs.
} 
map again, but this time in a $p$-adic context with $p \neq \ell$ (whereas Sch15b worked in the situation $p=\ell$ ). We note that this should make it possible to understand the behaviour of $\rho_{\mathfrak{m}}$ at places above $\ell$.

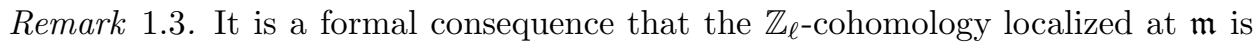
concentrated in degree $q_{0}$, and torsion-free, if the conclusion of the theorem holds true.

Remark 1.4. The condition that $\rho_{\mathfrak{m}}$ is decomposed generic is defined below. It follows from a suitable "big image" assumption. However, note that if $\rho_{\mathfrak{m}}$ is a generic sum of characters, there will still be a prime $p$ as in the theorem, so that our result also applies to many reducible representations.

Remark 1.5. We prove the result under a slightly weaker assumption depending on the precise signature of $G$. In particular, if the signature of $G$ is $(0, n)$ at all except for one infinite place, e.g. in the Harris-Taylor case, we only need the existence of one finite prime $v$ of $F$ at which $\rho_{\mathfrak{m}}$ is unramified and decomposed generic.

Remark 1.6. In the Harris-Taylor case (i.e., $G$ is of signature $(1, n-1)$ at one infinite place, and $(0, n)$ at the other places), there has been previous work on this question, notably by Shin, Shi15, restricting attention to the cohomology that is supercuspidal modulo $\ell$ at some finite prime $p$, by Emerton and Gee, EG15, making suitable assumptions on $\rho_{\mathfrak{m}}$ at $\ell$-adic places, and by Boyer, Boy15, under a condition very closely related to our condition.

Remark 1.7. Lan and Suh, LS12, prove that if the level is hyperspecial at $\ell$ and one takes cohomology with coefficients in the local system $\mathcal{L}_{\xi}$ corresponding to a suitably generic algebraic representation $\xi$ of $G$, then the whole $\ell$-adic cohomology groups $H^{i}\left(X_{\Gamma}, \mathcal{L}_{\xi}\right)$ for $i \neq q_{0}$ vanish. This behaviour cannot be expected in our situation, as at least all even cohomology groups $H^{2 i}\left(X_{\Gamma}, \mathbb{F}_{\ell}\right)$ are nonzero, so it is necessary to localize at some maximal ideal of the Hecke algebra.

Remark 1.7.1. An argument involving the Hochschild-Serre spectral sequence and Poincaré duality shows that the theorem also holds when $\mathbb{F}_{\ell}$ is replaced by a nontrivial (Hecke-equivariant) coefficient system.

Remark 1.8. Let $F$ be a $\mathrm{CM}$ field and $\Pi$ be a conjugate self-dual regular algebraic cuspidal automorphic representation of $G L_{n}\left(\mathbb{A}_{F}\right)$. Then $\Pi$ will be obtained by base change from an automorphic representation $\pi$ on a unitary group, which contributes to the cohomology of a compact unitary Shimura variety (see, for example, [HT01, Shi11, Car12]). In this situation, $\pi$ contributes only to the middle degree cohomology, and the proof relies on genericity rather than temperedness. In fact, concentration in middle degree is proved simultaneously with the RamanujanPetersson conjecture (at finite places) for $\Pi$ as above, using the template of [HT01] rather than appealing to $[\mathrm{BW} 80$. These results rely on the fact that the local components of cuspidal automorphic representations of $G L_{n}$ are generic, and follow by combining the classification of unitary generic representations of $G L_{n}$ due to Tadic (and the bounds of Jacquet-Shalika) with the Weil conjectures. While temperedness is an analytic condition, genericity can be formulated modulo $\ell$.

Let us define the critical notion of being decomposed generic.

Definition 1.9. Let $L$ be a $p$-adic field with residue field $\mathbb{F}_{q}, \ell \neq p$. An unramified representation

$$
\bar{\rho}: \operatorname{Gal}(\bar{L} / L) \rightarrow G L_{n}\left(\overline{\mathbb{F}}_{\ell}\right)
$$


is decomposed generic if the eigenvalues $\left\{\lambda_{1}, \ldots, \lambda_{n}\right\}$ of $\bar{\rho}$ (Frob), where Frob $\in$ $\operatorname{Gal}(\bar{L} / L)$ is an arithmetic Frobenius, satisfy $\lambda_{i} / \lambda_{j} \notin\{1, q\}$ for all $i \neq j$.

The main consequence of this definition is that any characteristic 0 lift of $\bar{\rho}$ is a direct sum of characters (i.e., "decomposed"), and the associated representation of $G L_{n}(L)$ under the local Langlands correspondence is a generid 3 principal series representation, cf. Lemma 6.2.2.

The rough idea. Let us now explain the idea of our proof. In very rough terms, the idea is to work at a fixed prime $p \neq \ell$, and look at the projection from the Shimura variety $S$, which is a moduli space of abelian varieties (with extra structures), to the corresponding moduli space $M$ of $p$-divisible groups (with extra structures),

$$
\pi: S \rightarrow M \text { 年 }
$$

One could then analyze the cohomology of the Shimura variety in terms of a Leray spectral sequence. Note that the fibres of $\pi$ should be a moduli space of abelian varieties with a trivialization of their $p$-divisible group, which are essentially the Igusa varieties of Man05, cf. also HT01. This means that one can compute the fibres of $R \pi_{*} \mathbb{Z}_{\ell}$ in terms of the cohomology of Igusa varieties. The alternating sum of the $\overline{\mathbb{Q}}_{\ell}$-cohomology groups has been analyzed in depth by Sug Woo Shin, [Shi09], Shi10.

An important property of the situation is that the Hecke operators away from $p$ act trivially on $M$, so the passage to the localization at $\mathfrak{m}$ can already be done on the sheaf $R \pi_{*} \mathbb{F}_{\ell}$. This makes it possible to use geometry on $M$. More specifically, in the actual setup considered below, (the localization at $\mathfrak{m}$ of) $R \pi_{*} \mathbb{F}_{\ell}$ will turn out to be perverse (up to shift), and thus is concentrated in one degree on the largest stratum where it is nonzero. In that case, (the localization at $\mathfrak{m}$ of) $R \pi_{*} \mathbb{Z}_{\ell}$ will be concentrated in one degree and flat. Thus, not much information is lost by passing to the alternating sum of the $\overline{\mathbb{Q}}_{\ell^{-}}$cohomology groups. Specifically, we will use this argument inductively to show that $\left(R \pi_{*} \mathbb{Z}_{\ell}\right)_{\mathfrak{m}}$ is trivial on all strata except the 0 -dimensional stratum, which will then give the desired bound.

Unfortunately, the moduli space $M$ of $p$-divisible groups does not really exist, or at the very least has horrible properties. This makes it hard to execute this strategy in a naive way. In April 2011, Sch11, one of us realized (in the HarrisTaylor case) that there should be a Hodge-Tate period map, which would make a

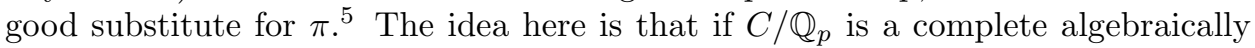
closed nonarchimedean field with ring of integers $\mathcal{O}_{C}$, then by [SW13, Theorem B], $p$-divisible groups over $\mathcal{O}_{C}$ are classified by pairs $(T, W)$, where $T$ is a finite free $\mathbb{Z}_{p}$-module, and $W \subset T \otimes_{\mathbb{Z}_{p}} C$ is a subvectorspace, the Hodge-Tate filtration. In particular, $p$-divisible groups with a trivialization of their Tate module are classified by a Grassmannian, at least on $\left(C, \mathcal{O}_{C}\right)$-valued points. Now, even if the moduli space of $p$-divisible groups is not a nice object, one can replace it by this Grassmannian, which is manifestly a nice object. It turns out that with this modification, the argument outlined above works.

The precise ideas. Let us now be more precise. We work adèlically, so for any compact open subgroup $K \subset G\left(\mathbb{A}_{f}\right)$, we have the Shimura variety $S_{K}$, which is a

\footnotetext{
${ }^{3}$ Recall that a generic representation is one which admits a Whittaker model - see, for example Section 2.3 of Kud94.

${ }^{4}$ This idea is also behind [Sch13b], and was also mentioned to one of us (P.S.) by R. Kottwitz.

${ }^{5}$ We learnt that L. Fargues had also been aware of the Hodge-Tate period map in some form.
} 
quasiprojective scheme over the reflex field $E$. For the moment, we allow an arbitrary Shimura variety. Recall that these are associated with Shimura data, which consist of a reductive group $G / \mathbb{Q}$ and a $G(\mathbb{R})$-conjugacy class $X$ of homomomorphisms $h: \operatorname{Res}_{\mathbb{C} / \mathbb{R}} \mathbb{G}_{m} \rightarrow G_{\mathbb{R}}$, subject to the usual axioms. Then

$$
S_{K}(\mathbb{C})=G(\mathbb{Q}) \backslash\left(X \times G\left(\mathbb{A}_{f}\right) / K\right) .
$$

Associated with any $h$, one has a minuscule cocharacter $\mu=\mu_{h}: \mathbb{G}_{m} \rightarrow G_{\mathbb{C}}$. The reflex field $E \subset \mathbb{C}$ is the field of definition of the conjugacy class of $\mu$. With any cocharacter $\mu$, one can associate two opposite parabolics $P_{\mu}$ and $P_{\mu}^{\text {std }}$, and there are two corresponding flag varieties $\mathrm{Fl}_{G, \mu}$ and $\mathrm{Fl}_{G, \mu}^{\text {std }}$ over $E$, parametrizing parabolic subgroups in the given conjugacy class. The association $h \mapsto \mu_{h} \mapsto P_{\mu_{h}}^{\text {std }}$ defines the (holomorphic) Borel embedding $X \hookrightarrow \mathrm{Fl}_{G, \mu}^{\text {std }}(\mathbb{C})$. There is also an antiholomorphic embedding $X \hookrightarrow \mathrm{Fl}_{G, \mu}(\mathbb{C})$ defined using $P_{\mu_{h}}$.

Fix any prime $p$, and $\mathfrak{p} \mid p$ a place of the reflex field $E$. Denote by $\mathcal{S}_{K}$ the rigidanalytic variety, or rather the adic space, corresponding to $S_{K} \otimes_{E} E_{\mathfrak{p}}$, and similarly for $\mathscr{F} \ell_{G, \mu}$. Our first main result refines the theory behind the Hodge-Tate period map from $\mathrm{Sch15b}$, which can be regarded as a $p$-adic version of the (antiholomorphic) Borel embedding.

Theorem 1.10. Assume that the Shimura datum is of Hodge type. Then for any sufficiently small compact open subgroup $K^{p} \subset G\left(\mathbb{A}_{f}^{p}\right)$, there is a perfectoid space $\mathcal{S}_{K^{p}}$ over $E_{\mathfrak{p}}$ such that

$$
\mathcal{S}_{K^{p}} \sim \lim _{K_{p}} \mathcal{S}_{K_{p} K^{p}}
$$

Moreover, there is a Hodge-Tate period map

$$
\pi_{H T}: \mathcal{S}_{K^{p}} \rightarrow \mathscr{F} \ell_{G, \mu}
$$

which agrees with the Hodge-Tate period map constructed in Sch15b for the Siegel case, and is functorial in the Shimura datum.

Moreover, we prove a result saying that all semisimple automorphic vector bundles come via pullback along $\pi_{H T}$.

The idea here is to chase Hodge tensors through all constructions, which is possible by using Deligne's results that they are absolute Hodge, Del82, (and also satisfy a compatibility under the $p$-adic comparison isomorphism, Bla94), and the results on relative $p$-adic Hodge theory of [Sch13c]. The details appear in Section 2 As stated above, one should think of $\mathscr{F} \ell_{G, \mu}$ as a (substitute for the) moduli space of $p$-divisible groups with extra structure and trivialized Tate module.

Next, we want to identify the fibres of $\pi_{H T}$ with Igusa varieties. First, we have to define a natural stratification on $\mathscr{F} \ell_{G, \mu}$, which correspondends under $\pi_{H T}$ to the Newton stratification (pulled back from the special fibre through the specialization map). Recall that the Newton strata are parametrized by the finite subset $B\left(G, \mu^{-1}\right) \subset B(G)$ of Kottwitz' set $B(G)$ of isocrystals with $G$-structure.

Theorem 1.11. Let $G$ be a reductive group over $\mathbb{Q}_{p}$, and $\mu$ a conjugacy class of minuscule cocharacters. There is a natural decomposition

$$
\mathscr{F} \ell_{G, \mu}=\bigsqcup_{b \in B\left(G, \mu^{-1}\right)} \mathscr{F} \ell_{G, \mu}^{b}
$$


into locally closed subsets $\mathscr{F}_{G, \mu}^{b}$. The union

$$
\bigsqcup_{b \preceq b^{\prime}} \mathscr{F} \ell_{G, \mu}^{b^{\prime}}
$$

is closed for all $b \in B\left(G, \mu^{-1}\right)$; in particular, $\mathscr{F}_{G, \mu}^{b}$ is open when $b$ is the basic element of $B\left(G, \mu^{-1}\right)$.

Thus, the closure relations are exactly the opposite of the closure relations of the Newton stratification on the Shimura variety 6 this change of closure relations is related to a subtle behaviour of $\pi_{H T}$ on certain higher-rank points of the adic space.

To give an idea of what the stratification looks like, we recall the example of the modular curve. In that case, the flag variety is just $\mathbb{P}^{1}$. The whole ordinary locus of the modular curve is contracted to $\mathbb{P}^{1}\left(\mathbb{Q}_{p}\right)$, and the Hodge-Tate period map just measures the position of the canonical subgroup on this locus. The supersingular locus is mapped onto Drinfeld's upper half-plane $\Omega^{2}=\mathbb{P}^{1} \backslash \mathbb{P}^{1}\left(\mathbb{Q}_{p}\right)$ in a way best understood using the isomorphism between the Lubin-Tate and Drinfeld towers. Thus, in this case the relevant stratification of $\mathbb{P}^{1}$ is simply the stratification into $\mathbb{P}^{1}\left(\mathbb{Q}_{p}\right)$ and $\Omega^{2}$. We caution the reader that in general, the strata $\mathscr{F} \ell_{G, \mu}^{b}$ are quite amorphous, and it happens that some nonempty strata have no classical points. The reason is that if $b$ is basic, $\mathscr{F} \ell_{G, \mu}^{b}$ agrees with the admissible locus in the sense of [RZ96], which does not admit a nice description, but whose classical points agree with the explicit weakly admissible locus. If $G$ is a non-split inner form of $G L_{5}$ and $\mu$ corresponds to $(1,1,0,0,0)$, one can verify that all classical points of $\mathscr{F} \ell_{G, \mu}$ are contained in the basic locus, while there are many other nonempty strata.

The proof of this theorem relies on certain recent advances in $p$-adic Hodge theory. First, to define the stratification on points, we make use of the classification of $G$-bundles on the Fargues-Fontaine curve; by a recent result of L. Fargues, Far15a, they are classified up to isomorphism by $B(G)$. Here, we construct a $G$-bundle on the Fargues-Fontaine curve by starting with the trivial $G$-bundle and modifying it at the infinite point of the Fargues-Fontaine curve. To construct the modification, we have to relate the flag variety $\mathscr{F} \ell_{G, \mu}$ to a Schubert cell in a $B_{\mathrm{dR}}^{+}$-affine Grassmannian as studied in Wei14; however, for our applications, the theory of Wei14] is not necessary.

Finally, to check the closure relations, we use recent results of Kedlaya and Liu, KL15, on the semicontinuity of the Newton polygon for families of $\varphi$-modules over the Robba ring.

Now we can relate the fibres of $\pi_{H T}$ to Igusa varieties. From now on, we assume that the Shimura variety is of PEL type (of type A or C), and compact, with good reduction at $p$. Pick any $b \in B\left(G, \mu^{-1}\right)$. Corresponding to $b$, we can find a $p$-divisible group $\mathbb{X}_{b}$ over $\overline{\mathbb{F}}_{p}$ equipped with certain extra endomorphism and polarization structures. We consider the following kind of Igusa varieties.

Proposition 1.12. There is a perfect scheme $\operatorname{Ig}^{b}$ over $\overline{\mathbb{F}}_{p}$ which parametrizes abelian varieties $A$ with extra structures, equipped with an isomorphism $\rho: A\left[p^{\infty}\right] \cong$ $\mathbb{X}_{b}$.

\footnotetext{
${ }^{6}$ We note that we do not prove that the closure of a stratum is a union of strata, so the term "closure relations" is meant in a loose sense.
} 
One can identify $\operatorname{Ig}^{b}$ with the perfection of the tower $\mathscr{I}_{\text {Mant }}^{b}=\varliminf_{m} \mathscr{I}_{\text {Mant }, m}^{b}$ of Igusa varieties constructed by Mantovan, Man05].

In particular, the étale cohomology of $\operatorname{Ig}^{b}$ agrees with the étale cohomology of Igusa varieties.

Let us also mention the following proposition. Here, $\mathscr{S}_{K}^{b} \subset \mathscr{S}_{K} \otimes \overline{\mathbb{F}}_{p}, b \in$ $B\left(G, \mu^{-1}\right)$ denotes a Newton stratum of the natural integral model $\mathscr{S}_{K}$ of the Shimura variety $S_{K}$ at hyperspecial level.

Proposition 1.13. Fix a geometric base point $\bar{x} \in \mathscr{S}_{K}^{b}$. There is a natural map

$$
\pi_{1}^{\text {proét }}\left(\mathscr{S}_{K}^{b}, \bar{x}\right) \rightarrow J_{b}\left(\mathbb{Q}_{p}\right),
$$

corresponding to a $J_{b}\left(\mathbb{Q}_{p}\right)$-torsor over $\mathscr{S}_{K}^{b}$ which above any geometric point parametrizes quasi-isogenies between $A\left[p^{\infty}\right]$ and $\mathbb{X}_{b}$ respecting the extra structures.

Remark 1.14. Here, $\pi_{1}^{\text {proét }}$ is the pro-étale fundamental group introduced in BS15a]. For normal schemes, it agrees with the usual profinite étale fundamental group of SGA1. However, Newton strata are usually not normal, and in fact the homomorphism to $J_{b}\left(\mathbb{Q}_{p}\right)$ often has noncompact image. For example, if $b$ is basic, then the image is a discrete cocompact subgroup of $J_{b}\left(\mathbb{Q}_{p}\right)$, related to the $p$-adic uniformization of the basic locus as in RZ96. Thus, the formalism of $\pi_{1}^{\text {proét }}$ is crucial for this statement.

Restricted to the leaf $\mathscr{C}_{b} \subset \mathscr{S}_{K}^{b}$ (the set of points where $A\left[p^{\infty}\right] \cong \mathbb{X}_{b}$ ), the map $\pi_{1}\left(\mathscr{C}_{b}, \bar{x}\right) \rightarrow J_{b}\left(\mathbb{Q}_{p}\right)$ takes values in a compact open subgroup of $J_{b}\left(\mathbb{Q}_{p}\right)$, and then corresponds to the tower of finite étale covers $\mathscr{I}_{\text {Mant,m }}^{b} \rightarrow \mathscr{C}_{b}$ considered by Mantovan.

There is a close relation between the fibres of $\pi_{H T}$ over points in $\mathscr{F}_{G, \mu}^{b}$ and the perfect schemes $\operatorname{Ig}^{b}$; note however that the former are of characteristic 0 while the latter are of characteristic $p$. Roughly, one is the canonical lift of the other, except for issues of higher rank points. In any case, one gets the following cohomological consequence.

Theorem 1.15. Let $\bar{x}$ be any geometric point of $\mathscr{F} \ell_{G, \mu}^{b} \subset \mathscr{F} \ell_{G, \mu}$. For any $\ell \neq p$, there is an isomorphism

$$
\left(R \pi_{H T *} \mathbb{Z} / \ell^{n} \mathbb{Z}\right)_{\bar{x}} \cong R \Gamma\left(\operatorname{Ig}^{b}, \mathbb{Z} / \ell^{n} \mathbb{Z}\right)
$$

compatible with the Hecke action of $G\left(\mathbb{A}_{f}^{p}\right)$.

We recall that the alternating sum of the $\overline{\mathbb{Q}}_{\ell}$-cohomology of Igusa varieties has been computed by Sug Woo Shin, Shi09, Shi10. His results are presented in Section 5 and combined with the (twisted) trace formula.

The final ingredient necessary for the argument as outlined above is that $R \pi_{H T *} \mathbb{F}_{\ell}$ is perverse. Obviously, $R \pi_{H T *} \mathbb{F}_{\ell}$ should be constructible with respect to the stratification

$$
\mathscr{F} \ell_{G, \mu}=\bigsqcup_{b \in B\left(G, \mu^{-1}\right)} \mathscr{F} \ell_{G, \mu}^{b} .
$$

However, as the strata are amorphous, it is technically difficult to define a notion of perverse sheaf in this setup. We content ourselves here with proving just what is necessary for us to conclude. Specifically, we will prove that the $K_{p}$-invariants of 
the nearby cycles of $R \pi_{H T *} \mathbb{F}_{\ell}$ are perverse, for any formal model of $\mathscr{F} \ell_{G, \mu}$ and sufficiently small compact open subgroup $K_{p} \subset G\left(\mathbb{Q}_{p}\right)$. Choosing these formal models correctly will then make it possible to deduce that the cohomology is concentrated in one degree on the largest stratum where it is nonzero.

Remark 1.16. Heuristically, the reason that $R \pi_{H T *} \mathbb{F}_{\ell}$ is perverse is that $\pi_{H T}$ is simultaneously affine and partially proper (i.e., satisfies the valuative criterion of properness). In classical algebraic geometry, this would mean that $\pi_{H T}$ is finite, and pushforward along finite morphisms preserves perversity. In general, partially proper implies that $R \pi_{H T *}=R \pi_{H T \text { ! }}$, so assuming that there is a Verdier duality which exchanges these two functors, one has to prove only one of the two support inequalities defining a perverse sheaf. This inequality is precisely Artin's bound on the cohomological dimension of affine morphisms.

Remark 1.17. The fact that the closure relations are reversed on the flag variety is critical to our strategy. Namely, our assumption on $\rho_{\mathfrak{m}}$ ensures that the cohomology should be "maximally ordinary", and this makes it reasonable to hope that everything comes from the $\mu$-ordinary locus. In our setup, the $\mu$-ordinary locus inside the flag variety is the closed stratum, and 0 -dimensional. In the naive moduli space of $p$-divisible groups, the $\mu$-ordinary locus would be open and dense (cf. Wed99]), and the inductive argument outlined above would stop at the first step.

Remark 1.18. Recently, L. Fargues, Far, has conjectured that to any local $L$ parameter, there is a corresponding perverse sheaf on the stack Bun ${ }_{G}$ of $G$-bundles on the Fargues-Fontaine curve, thus realizing the local Langlands correspondence as a geometric Langlands correspondence on the Fargues-Fontaine curve. We conjecture that the perverse sheaves $R \pi_{H T *} \overline{\mathbb{Q}}_{\ell}$ on $\mathscr{F} \ell_{G, \mu}$ are related to these conjectural perverse sheaves on $\operatorname{Bun}_{G}$ via pullback along the natural map $\mathscr{F} \ell_{G, \mu} \rightarrow \mathrm{Bun}_{G}$, by some form of local-global compatibility. In the Harris-Taylor case, one can be more explicit, and this was the subject of Sch11].

Acknowledgments. First, we wish to thank J.-F. Dat for many discussions on the "geometrization" of the results of Sch13b using perverse sheaves on the moduli space of $p$-divisible groups. The rough strategy of this argument was first explained during a workshop on Barbados in May 2014, and we want to thank the organizers for the chance to present these ideas there. Moreover, we want to thank F. Calegari, L. Fargues, K. Kedlaya and S. W. Shin for many helpful discussions, and C.-L. Chai for sending us a preliminary version of his work with F. Oort on the "internal Hom $p$-divisible group". Part of this work was completed while both authors attended the special program on "New geometric methods in number theory" at MSRI in Fall 2014; we thank the institute and the organizers for the excellent working atmosphere. During that time, P. Scholze held a Chancellor's Professorship at UC Berkeley. This work was done while P. Scholze was a Clay Research Fellow.

Notation and conventions. A nonarchimedean field $K$ is a topological field whose topology is induced by a continuous rank 1 valuation (which is necessarily uniquely determined, up to equivalence). We denote by $\mathcal{O}_{K} \subset K$ the subring of powerbounded elements, which is the set of element of absolute value $\leq 1$ under the rank 1 valuation. If, in the context of adic spaces, $K$ is equipped with a higher rank valuation, we denote by $K^{+} \subset \mathcal{O}_{K}$ the open and bounded valuation subring of elements which are $\leq 1$ for this higher rank valuation. 
We have tried our best to make our signs internally consistent, although the reader may often feel the presence of unnecessarily many minus signs. As regards slopes, we observe the following. We use covariant Dieudonné theory. Usually, this sends $\mathbb{Q}_{p} / \mathbb{Z}_{p}$ to $\left(\mathbb{Z}_{p}, F=p\right)$ and $\mu_{p^{\infty}}$ to $\left(\mathbb{Z}_{p}, F=1\right)$; this is, however, not compatible with passage to higher tensors. The underlying reason is that in the duality between covariant and contravariant Dieudonné theory, there is an extra Tate twist; for this reason, we divide the usual Frobenius by $p$, which gets rid of this Tate twist. Thus, the covariant Dieudonné module for $\mu_{p \infty}$ is $\left(\mathbb{Z}_{p}, F=p^{-1}\right)$ in our setup, and one sees that the Frobenius operator does not preserve the lattice; in general, the associated Dieudonné module will have slopes in $[-1,0]$. However, in the passage from isocrystals to vector bundles on the Fargues-Fontaine curve, the isocrystal $\left(\mathbb{Q}_{p}, F=p^{-1}\right)$ is sent to the ample line bundle $\mathcal{O}(1)$, so the slope changes sign once more, and in the end the usual slope of a $p$-divisible group agrees with the slope of the associated vector bundle on the Fargues-Fontaine curve. We feel that any confusion about signs on this part of the story is inherent to the mathematics involved.

As regards cocharacters (and associated filtrations), we have adopted what we think is the standard definition of the cocharacter $\mu=\mu_{h}$ corresponding to a Shimura datum $\{h\}$; for example, in the case of the modular curve, $\mu(t)=\operatorname{diag}(t, 1)$ as a map $\mathbb{G}_{m} \rightarrow G L_{2}$. This has the advantage of being "positive", but the disadvantage that virtually everywhere we have to consider $\mu^{-1}$ instead; e.g., with this normalization, it is the set $B\left(G, \mu^{-1}\right)$ which parametrizes the Newton strata. We feel that on this side of the story, it might be a good idea to exchange $\mu$ by $\mu^{-1}$, but we have stuck with the standard choice. 


\section{Refining the Hodge-Tate Period maP}

In this section, we work with a general Shimura variety of Hodge type and we prove that the Hodge-Tate period map from the corresponding perfectoid Shimura variety factors through the expected flag variety.

2.1. Recollections on the Hodge-Tate period map. Let $(G, X)$ be a Shimura datum, where $X$ is a $G(\mathbb{R})$-conjugacy class of homomorphisms

$$
h: \operatorname{Res}_{\mathbb{C} / \mathbb{R}} \mathbb{G}_{m} \rightarrow G_{\mathbb{R}} .
$$

Recall that $(G, X)$ is a Shimura datum if it satisfies the following three conditions:

(1) Let $\mathfrak{g}$ denote the Lie algebra of $G(\mathbb{R})$. For any choice of $h \in X$, its composition with the adjoint action of $G(\mathbb{R})$ on $\mathfrak{g}$ determines a Hodge structure of type $(-1,1),(0,0),(1,-1)$ on $\mathfrak{g} \cdot \sqrt{7}$

(2) $h(i)$ is a Cartan involution of $G^{\text {ad }}(\mathbb{R})$;

(3) $G^{\text {ad }}$ has no factor defined over $\mathbb{Q}$ whose real points form a compact group. The second condition implies that the stabilizer of any $h$ is compact modulo its center.

A choice of cocharacter $h$ determines, via base change to $\mathbb{C}$ and restriction to the first $\mathbb{G}_{m}$ factor, a Hodge cocharacter $\mu: \mathbb{G}_{m} \rightarrow G_{\mathbb{C}}$. This allows us to define two opposite parabolic subgroups:

$$
\begin{gathered}
P_{\mu}^{\text {std }}:=\left\{g \in G \mid \lim _{t \rightarrow \infty} \operatorname{ad}(\mu(t)) g \text { exists }\right\}, \text { and } \\
P_{\mu}:=\left\{g \in G \mid \lim _{t \rightarrow 0} \operatorname{ad}(\mu(t)) g \text { exists }\right\} .
\end{gathered}
$$

The Hodge cocharacter $\mu$ defines a filtration on the category $\operatorname{Rep}_{\mathbb{C}}(G)$ of finitedimensional representations of $G$ on $\mathbb{C}$-vector spaces. Indeed, the action of $\mathbb{G}_{m}$ on $\operatorname{Rep}_{\mathbb{C}}(G)$ via $\mu$ induces a grading on $\operatorname{Rep}_{\mathbb{C}}(G)$ and we take $\operatorname{Fil}^{\bullet}(\mu)$ to be the descending filtration on $\operatorname{Rep}_{\mathbb{C}}(G)$ associated with this grading. Concretely, $\operatorname{Fil}^{p}(\mu)$ is the direct sum of all subspaces of type $\left(p^{\prime}, q^{\prime}\right)$ with $p^{\prime} \geq p$. The parabolic $P_{\mu}^{\text {std }}$ can equivalently be defined as the subgroup of $G$ stabilizing Fil $^{\bullet}(\mu)$. The opposite parabolic $P_{\mu}$ can be defined as the stabilizer of the opposite, ascending filtration Fil. $(\mu)$, where $\operatorname{Fil}_{p}(\mu)$ is the direct sum of all subspaces of type $\left(p^{\prime}, q^{\prime}\right)$ with $p^{\prime} \leq p$. Both conjugacy classes of parabolics are defined over the reflex field $E$ of the Shimura datum, which is the minimal field of definition of the conjugacy class $\{\mu\}$. Note that

$$
M_{\mu}:=\operatorname{Cent}_{G}(\mu)
$$

is the Levi component of both parabolics.

The two parabolics determine two flag varieties $\mathrm{Fl}_{G, \mu}^{\mathrm{std}}$ and $\mathrm{Fl}_{G, \mu}$ over $E$ parametrizing parabolics in the given conjugacy class. The choice of a base point $h$ allows us to identify $\mathrm{Fl}_{G, \mu}^{\mathrm{std}}(\mathbb{C}) \simeq G(\mathbb{C}) / P_{\mu}^{\text {std }}(\mathbb{C})$. There is an embedding

$$
\beta: X \hookrightarrow \mathrm{Fl}_{G, \mu}^{\mathrm{std}}(\mathbb{C})
$$

called the Borel embedding, defined by $h \mapsto \operatorname{Fil}^{\bullet}\left(\mu_{h}\right)$. It is easy to see that the Borel embedding is holomorphic. (There is also an embedding

$$
X \hookrightarrow \mathrm{Fl}_{G, \mu}(\mathbb{C}),
$$

\footnotetext{
${ }^{7}$ Here, an action of $\mathbb{C}^{*}$ on a $\mathbb{C}$-vector space is said to be of type $\left\{\left(p_{i}, q_{i}\right)\right\}$ if the vector space decomposes as a direct sum of subspaces, on which the action is through the cocharacters $z \mapsto$ $z^{-p_{i}} \bar{z}^{-q_{i}}$
} 
which is antiholomorphic, defined in the natural way from the opposite filtration Fil. $(\mu)$.)

Let $K \subset G\left(\mathbb{A}_{f}\right)$ be a compact open subgroup. Let

$$
S_{K}(\mathbb{C}):=G(\mathbb{Q}) \backslash\left(X \times G\left(\mathbb{A}_{f}\right) / K\right) .
$$

When $K$ is neat (so, when $K$ is small enough), $S_{K}(\mathbb{C})$ has the structure of an algebraic variety over $\mathbb{C}$ (by a theorem of Baily-Borel) and has a model $S_{K}$ over the reflex field $E$ Mil90.

Example 2.1.1. Let $g \geq 1$ and let

$$
(V, \psi)=\left(\mathbb{Q}^{2 g}, \psi\left(\left(a_{i}\right),\left(b_{i}\right)\right)=\sum_{i=1}^{g}\left(a_{i} b_{g+i}-a_{g+i} b_{i}\right)\right)
$$

be the split symplectic space of dimension $2 g$ over $\mathbb{Q}$. Let $\tilde{G}:=G S p(V, \psi)$. The hermitian symmetric domain $\tilde{X}$ is the Siegel double space. Fix the self-dual lattice $\Lambda=\mathbb{Z}^{2 g}$ in $V$. For every $h \in \tilde{X}$, the Hodge structure induced by $\mu_{h}$ on $V$ has type $(-1,0),(0,-1)$ and $V^{(-1,0)} / \Lambda$ is an abelian variety over $\mathbb{C}$ of dimension $g$.

For $\tilde{K} \subset \tilde{G}\left(\mathbb{A}_{f}\right)$ a neat compact open subgroup, the corresponding Shimura variety $\tilde{S}_{\tilde{K}}$ is the moduli space of principally polarized $g$-dimensional abelian varieties with level- $\tilde{K}$-structure. It has a model over the reflex field $\mathbb{Q}$. It carries a universal abelian variety $\mathcal{A}$ and a natural ample line bundle $\omega$ given by the determinant of the sheaf of invariant differentials on $\mathcal{A}$. The flag variety $\mathrm{Fl}_{\tilde{G}, \tilde{\mu}}$ parametrizes totally isotropic subspaces $W \subset V$.

We say that a Shimura datum is of Hodge type if it admits a closed embedding $(G, X) \hookrightarrow(\tilde{G}, \tilde{X})$, for some choice of Siegel data $(\tilde{G}, \tilde{X})$. A consequence of this is that the associated Shimura variety $S_{K}$ (for some neat level $K$ ) carries a universal abelian variety, which is the restriction of the universal abelian variety over $\tilde{S}_{\tilde{K}}$. One can regard $S_{K}$ as a moduli space for abelian varieties equipped with certain Hodge tensors, cf. below.

Let $(G, X)$ be a Shimura datum of Hodge type and let $(\tilde{G}, \tilde{X})$ be a choice of Siegel data, for which there exists an embedding $(G, X) \hookrightarrow(\tilde{G}, \tilde{X})$. Fixing such an embedding gives rise to closed embeddings $\mathrm{Fl}_{G, \mu}^{(\mathrm{std})} \hookrightarrow \mathrm{Fl}_{\tilde{G}, \tilde{\mu}}^{\text {(std) }}$. By [Del71, Proposition 1.15], there exists some compact open subgroup $\tilde{K} \subset \tilde{G}\left(\mathbb{A}_{f}\right)$ with $K=\tilde{K} \cap G\left(\mathbb{A}_{f}\right)$ such that there is a closed embedding of the corresponding Shimura varieties over $E$,

$$
S_{K} \hookrightarrow \tilde{S}_{\tilde{K}} \otimes_{\mathbb{Q}} E
$$

Let $p$ be a prime number. We will consider compact open subgroups of the forms $K=K^{p} \times K_{p} \subset G\left(\mathbb{A}_{f}^{p}\right) \times G\left(\mathbb{Q}_{p}\right)$, where $K^{p}$ and $K_{p}$ are compact open. Fix a place $\mathfrak{p}$ of $E$ above $p$. Let $\mathscr{F} \ell_{G, \mu}$ be the adic space associated with $\mathrm{Fl}_{G, \mu} \otimes_{E} E_{\mathfrak{p}}$. The following is part of Theorem IV.1.1 of Sch15b 8

Theorem 2.1.2. $\quad$ (1) For any sufficiently small tame level $K^{p} \subset G\left(\mathbb{A}_{f}^{p}\right)$, there exists a perfectoid space $\mathcal{S}_{K^{p}}$ over $E_{\mathfrak{p}}$, such that

$$
\mathcal{S}_{K^{p}} \sim \lim _{K_{p}}\left(S_{K^{p} K_{p}} \otimes_{E} E_{\mathfrak{p}}\right)^{\mathrm{ad}}
$$

\footnotetext{
${ }^{8}$ The setup is slightly different, but the proof works verbatim.
} 
(2) There exists a $G\left(\mathbb{Q}_{p}\right)$-equivariant Hodge-Tate period map

$$
\pi_{H T}: \mathcal{S}_{K p} \rightarrow \mathscr{F}_{\tilde{G}, \tilde{\mu}} .
$$

(3) The map $\pi_{H T}$ is equivariant with respect to the natural Hecke action of $G\left(\mathbb{A}_{f}^{p}\right)$ on the inverse system of $\mathcal{S}_{K^{p}}$ and the trivial action of $G\left(\mathbb{A}_{f}^{p}\right)$ on $\mathscr{F} \ell_{\tilde{G}, \tilde{\mu}}$.

Recall that the Hodge-Tate period map Sch15b, SW13 has the following description on points: for $A / C$ an abelian variety of dimension $g$, the Tate module of $A$ admits the Hodge-Tate decomposition:

$$
0 \rightarrow(\operatorname{Lie} A)(1) \rightarrow T_{p} A \otimes_{\mathbb{Z}_{p}} C \rightarrow\left(\text { Lie } A^{\vee}\right)^{\vee} \rightarrow 0 .
$$

A point $x \in \mathcal{S}_{G, K^{p}}\left(C, C^{+}\right)$corresponding to $A / C$ together with a symplectic isomorphism $T_{p} A \stackrel{\sim}{\rightarrow} \mathbb{Z}_{p}^{2 g}$ (and extra structures) is mapped to the point $\pi_{H T}(x) \in$ $\mathscr{F} \ell_{\tilde{G}, \tilde{\mu}}\left(C, C^{+}\right)$corresponding to the Hodge-Tate filtration $\operatorname{Lie}(A) \subset C^{2 g}$.

We note that one can think of the Hodge-Tate period map as a $p$-adic analogue of the Borel embedding. The goal of this section is to prove the following theorem.

Theorem 2.1.3. (1) The Hodge-Tate period map for $\mathcal{S}_{K^{p}}$ factors through $\mathscr{F} \ell_{G, \mu}$ and the resulting map

$$
\pi_{H T}: \mathcal{S}_{K^{p}} \rightarrow \mathscr{F} \ell_{G, \mu}
$$

is independent of the choice of embedding of Shimura data.

(2) Fix some $\mu$ in the given conjugacy class, defined over a finite extension of $E$. The tensor functor from $\operatorname{Rep} M_{\mu}$ to $G\left(\mathbb{Q}_{p}\right)$-equivariant vector bundles on $\mathcal{S}_{K^{p}}$ given as the composition

$$
\begin{aligned}
f_{p}: \operatorname{Rep} M_{\mu} \hookrightarrow \operatorname{Rep} P_{\mu} & \longrightarrow\left\{G\left(\mathbb{Q}_{p}\right) \text {-equivariant vector bundles on } \mathscr{F} \ell_{G, \mu}\right\} \\
& \stackrel{\pi_{H T}^{*}}{\longrightarrow}\left\{G\left(\mathbb{Q}_{p}\right) \text {-equivariant vector bundles on } \mathcal{S}_{K^{p}}\right\}
\end{aligned}
$$

is isomorphic to the tensor functor

$$
\begin{aligned}
f_{\infty}: \operatorname{Rep} M_{\mu} \hookrightarrow \operatorname{Rep} P_{\mu}^{\text {std }} & \longrightarrow\left\{\text { automorphic vector bundles on } S_{K}\right\} \\
& \longrightarrow\left\{G\left(\mathbb{Q}_{p}\right) \text {-equivariant vector bundles on } \mathcal{S}_{K^{p}}\right\} .
\end{aligned}
$$

The isomorphism is independent of the choice of Siegel embedding, and equivariant for the Hecke action of $G\left(\mathbb{A}_{f}^{p}\right)$.

Remark 2.1.4. One may avoid choosing $\mu$ by replacing Rep $M_{\mu}$ with the category of $G$-equivariant vector bundles on the space of cocharacters in the conjugacy class of $\mu$. Note that after fixing any $\mu$, this space identifies with $G / M_{\mu}$, and so $G$ equivariant vector bundles are identified with representations of $M_{\mu}$. We leave it as an exercise to the reader to reformulate the theorem and its proof in this more canonical language.

Let us first recall how the tensor functor $f_{\infty}$ is defined: any representation $\xi$ of $M_{\mu}$ determines a representation of $P_{\mu}^{\text {std }}$ by making the unipotent radical act trivially. Now, starting with a representation of $P_{\mu}^{\text {std }}$, we can define an automorphic vector bundle on $S_{K}$ as in Section III of [Mil90], provided that the level $K$ is sufficiently small: first, there is an equivalence of categories

$$
\xi \mapsto \mathcal{W}(\xi)
$$


between $\operatorname{Rep}_{\mathbb{C}}\left(P_{\mu}^{\text {std }}\right)$ and the category of $G_{\mathbb{C}}$-equivariant vector bundles on $\mathrm{Fl}_{G, \mu}^{\text {std }}$ (the functor in one direction is taking the stalk of the vector bundle above the point corresponding to $\mu$ ). Then restriction along the image of the Borel embedding gives a $G(\mathbb{R})$-equivariant vector bundle on $X$. Passing to the double quotient defining the Shimura variety

$$
S_{K}(\mathbb{C})=G(\mathbb{Q}) \backslash\left(X \times G\left(\mathbb{A}_{f}\right) / K\right)
$$

over $\mathbb{C}$ defines the automorphic vector bundle

$$
\mathcal{V}(\xi):=G(\mathbb{Q}) \backslash\left(\mathcal{W}(\xi) \times G\left(\mathbb{A}_{f}\right) / K\right) .
$$

The automorphic vector bundles $\mathcal{V}(\xi)$ are algebraic and, when the representation $\xi$ is defined over a finite extension $E^{\prime}$ of $E, \mathcal{V}(\xi)$ is also defined over $E^{\prime}$.

Remark 2.1.5. Proving that the automorphic vector bundles descend to the reflex field makes use of an intermediate algebraic object between $S_{K}$ and $\mathrm{Fl}_{G, \mu}^{\text {std }}$, called the standard principal bundle (see Section IV of [Mil90]), which is a $G$-torsor over $S_{K}$. See the proof of Lemma 2.3.5 for more details.

In particular, $f_{p}$ is defined in an analogous way to $f_{\infty}$, except that it uses the Hodge-Tate period map in place of the Borel embedding. The appearance of the opposite parabolic $P_{\mu}$ in this picture forces one to look only at representations inflated from the common Levi $M_{\mu}$.

2.2. The $p$-adic-de Rham comparison isomorphism. For an abelian variety over $C$, its image under the Hodge-Tate period map is determined by the HodgeTate filtration on $H_{\text {ét }}^{1}\left(A, \mathbb{Q}_{p}\right) \otimes_{\mathbb{Q}_{p}} C$. The Hodge-Tate period map as a map of adic spaces $\mathcal{S}_{K^{p}} \rightarrow \mathscr{F}_{\tilde{G}, \tilde{\mu}}$ is defined via a relative version of the Hodge-Tate filtration, which is a filtration on the local system given by the $p$-adic étale cohomology of the universal abelian variety over $\mathcal{S}_{K}$, tensored with the completed structure sheaf of the base. In fact, the Hodge-Tate filtration is defined more generally: see Section 3 of $[\mathrm{Sch12b}$ for a construction of the Hodge-Tate filtration for a proper smooth rigid-analytic variety over a geometric point.

As we will need to work with higher tensors in our analysis of Hodge type Shimura varieties, our goal in this section is to give a construction of the relative Hodge-Tate filtration in the case of a proper smooth morphism $\pi: X \rightarrow S$ of smooth adic spaces over $\operatorname{Spa}\left(K, \mathcal{O}_{K}\right)$, where $K$ is a complete discretely valued field of characteristic 0 with perfect residue field $k$ of characteristic $p$. This will be done in a way that also clarifies its relationship to the relative $p$-adic-de Rham comparison isomorphism.

The following sheaves on $X_{\text {proét }}$ are defined in Sch13c: the completed structure sheaf $\hat{\mathcal{O}}_{X}$, the tilted completed structure sheaf $\hat{\mathcal{O}}_{X^{b}}$, the relative period sheaves $\mathbb{B}_{\mathrm{dR}, X}^{+}$and $\mathbb{B}_{\mathrm{dR}, X}$ as well as the structural de Rham sheaves $\mathcal{O} \mathbb{B}_{\mathrm{dR}, X}^{+}$and $\mathcal{O} \mathbb{B}_{\mathrm{dR}, X}$. We recall some of their definitions: the tilted integral structure sheaf $\hat{\mathcal{O}}_{X^{b}}^{+}$is the (inverse) perfection of $\hat{\mathcal{O}}_{X}^{+} / p$ (i.e., the inverse limit of $\hat{\mathcal{O}}_{X}^{+} / p$ with respect to the Frobenius morphism).

Definition 2.2.1. $\quad$ (1) The relative period sheaf $\mathbb{B}_{\mathrm{dR}, X}^{+}$is the completion of $W\left(\hat{\mathcal{O}}_{X^{b}}^{+}\right)[1 / p]$ along the kernel of the natural map $\theta: W\left(\hat{\mathcal{O}}_{X^{b}}^{+}\right)[1 / p] \rightarrow \hat{\mathcal{O}}_{X}$.

(2) The relative period sheaf $\mathbb{B}_{\mathrm{dR}, X}$ is $\mathbb{B}_{\mathrm{dR}, X}^{+}\left[\xi^{-1}\right]$, where $\xi$ is any element that generates the kernel of $\theta$. 
Lemma 6.3 of [Sch13c] shows that $\xi$ exists proétale locally on $X$, is not a zero divisor and is unique up to a unit. Therefore, the sheaf $\mathbb{B}_{\mathrm{dR}, X}$ is well-defined. When $X=\operatorname{Spa}\left(C, \mathcal{O}_{C}\right)$, we recover Fontaine's period $\operatorname{ring} B_{\mathrm{dR}, C}$ from this construction. By construction, the relative period sheaf $\mathbb{B}_{\mathrm{dR}, X}$ is equipped with a natural filtration $\mathrm{Fil}^{i} \mathbb{B}_{\mathrm{dR}, X}=\xi^{i} \mathbb{B}_{\mathrm{dR}, X}^{+}$, with $\mathrm{gr}^{0} \mathbb{B}_{\mathrm{dR}, X}=\hat{\mathcal{O}}_{X}$.

Recall that $k$ is the residue field of $K$. Then $\mathcal{O}_{X} \otimes_{W(k)} W\left(\hat{\mathcal{O}}_{X^{b}}\right)$ also admits a map $\theta: \mathcal{O}_{X} \otimes_{W(k)} W\left(\hat{\mathcal{O}}_{X^{b}}\right) \rightarrow \hat{\mathcal{O}}_{X}$. Then $\mathcal{O B}_{\mathrm{dR}}^{+}$is defined as the completion of $\mathcal{O}_{X} \otimes_{W(k)} W\left(\hat{\mathcal{O}}_{X^{b}}\right)$ along $\operatorname{ker} \theta$ and $\mathcal{O B}_{\mathrm{dR}}:=\mathcal{O B}_{\mathrm{dR}}^{+}\left[\xi^{-1}\right]$ as above. The structural de Rham sheaves $\mathcal{O B}(+)$ are equipped with filtrations and connections

$$
\nabla: \mathcal{O} \mathbb{B}_{\mathrm{dR}, X}^{(+)} \rightarrow \mathcal{O B}_{\mathrm{dR}, X}^{(+)} \otimes_{\mathcal{O}_{X}} \Omega_{X}^{1} .
$$

We have an identification $\left(\mathcal{O} \mathbb{B}_{\mathrm{dR}}^{(+)}\right)^{\nabla=0}=\mathbb{B}_{\mathrm{dR}}^{(+)}$.

We now recall the relative $p$-adic-de Rham comparison isomorphism for a proper smooth morphism $\pi: X \rightarrow S$ of smooth adic spaces over $K$.

Theorem 2.2.2 ([Sch13c, Theorem 8.8]). Assume that $R^{i} \pi_{*} \mathbb{F}_{p}$ is locally free on

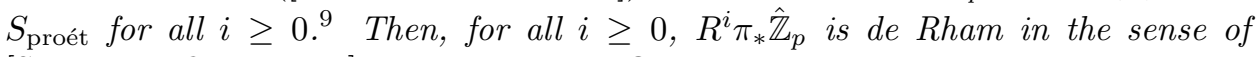
Sch13c, Definition 7.5], with associated filtered module with integrable connection given by $R^{i} \pi_{\mathrm{dR} *} \mathcal{O}_{X}$ (with its Hodge filtration, and Gauss-Manin connection). In particular, there is an isomorphism

$$
R^{i} \pi_{*} \hat{\mathbb{Z}}_{p, X} \otimes_{\hat{\mathbb{Z}}_{p, S}} \mathcal{O} \mathbb{B}_{\mathrm{dR}, S} \simeq R^{i} \pi_{\mathrm{dR} *} \mathcal{O}_{X} \otimes_{\mathcal{O}_{S}} \mathcal{O} \mathbb{B}_{\mathrm{dR}, S}
$$

of sheaves on $S_{\text {proét }}$, compatible with filtrations and connections.

Moreover, we need to recall the two different $\mathbb{B}_{\mathrm{dR}}^{+}$-local systems associated with $R^{i} \pi_{*} \hat{\mathbb{Z}}_{p}$. The first one, which is closely related to étale cohomology, is given by

$$
\mathbb{M}=R^{i} \pi_{*} \hat{\mathbb{Z}}_{p, X} \otimes_{\hat{\mathbb{Z}}_{p, S}} \mathbb{B}_{\mathrm{dR}, S}^{+} \cong R^{i} \pi_{*} \mathbb{B}_{\mathrm{dR}, X}^{+} .
$$

The other one, which is closely related to de Rham cohomology, is given by

$$
\mathbb{M}_{0}=\left(R^{i} \pi_{\mathrm{dR} *} \mathcal{O}_{X} \otimes_{\mathcal{O}_{S}} \mathcal{O} \mathbb{B}_{\mathrm{dR}, S}^{+}\right)^{\nabla=0} \text {. }
$$

Note that the definition of $\mathbb{M}_{0}$ did not make use of the Hodge filtration. The relation between these two lattices is given by the following proposition, which reformulates the condition of being associated.

Proposition 2.2.3 ([Sch13c, Proposition 7.9]). There is a canonical isomorphism

$$
\mathbb{M} \otimes_{\mathbb{B}_{\mathrm{dR}, S}^{+}} \mathbb{B}_{\mathrm{dR}, S} \cong \mathbb{M}_{0} \otimes_{\mathbb{B}_{\mathrm{dR}, S}^{+}} \mathbb{B}_{\mathrm{dR}, S}
$$

Moreover, for any $j \in \mathbb{Z}$, one has an identification

$$
\begin{aligned}
\left(\mathbb{M} \cap \mathrm{Fil}^{j} \mathbb{M}_{0}\right) /\left(\mathbb{M} \cap \mathrm{Fil}^{j+1} \mathbb{M}_{0}\right) & =\left(\mathrm{Fil}^{-j} R^{i} \pi_{\mathrm{dR} *} \mathcal{O}_{X}\right) \otimes_{\mathcal{O}_{S}} \hat{\mathcal{O}}_{S}(j) \\
\subset \operatorname{gr}^{j} \mathbb{M}_{0} & =R^{i} \pi_{\mathrm{dR} *} \mathcal{O}_{X} \otimes_{\mathcal{O}_{S}} \hat{\mathcal{O}}_{S}(j) .
\end{aligned}
$$

In particular, $\mathbb{M}_{0} \subset \mathbb{M}$.

\footnotetext{
${ }^{9}$ This condition is verified if $\pi$ is algebraizable, and has been announced in general by Gabber. Another proof will appear in a forthcoming version of [Wei14]; the idea is to use (the new version of) pro-étale descent to reduce to the case where $S$ is w-strictly local, in which case one can redo the finiteness argument over a geometric point. With $\mathbb{Q}_{p}$-coefficients, it has also been announced by Kedlaya-Liu.
} 
In particular, we get an ascending filtration on

$$
\operatorname{gr}^{0} \mathbb{M}=R^{i} \pi_{*} \hat{\mathbb{Z}}_{p, X} \otimes_{\hat{\mathbb{Z}}_{p, S}} \hat{\mathcal{O}}_{S}
$$

given by

$$
\mathrm{Fil}_{-j}\left(R^{i} \pi_{*} \hat{\mathbb{Z}}_{p, X} \otimes_{\hat{\mathbb{Z}}_{p, S}} \hat{\mathcal{O}}_{S}\right)=\left(\mathbb{M} \cap \mathrm{Fil}^{j} \mathbb{M}_{0}\right) /\left(\mathrm{Fil}^{1} \mathbb{M} \cap \mathrm{Fil}^{j} \mathbb{M}_{0}\right)
$$

Here, $\mathrm{Fil}_{-1}=0$, and $\mathrm{Fil}_{i}$ is everything. We call this filtration the relative HodgeTate filtration.

Corollary 2.2.4. For all $j \geq 0$, there are canonical isomorphisms

$$
\operatorname{gr}_{j}\left(R^{i} \pi_{*} \hat{\mathbb{Z}}_{p, X} \otimes_{\hat{\mathbb{Z}}_{p, S}} \hat{\mathcal{O}}_{S}\right) \cong\left(\operatorname{gr}^{j} R^{i} \pi_{\mathrm{dR} *} \mathcal{O}_{X}\right) \otimes_{\mathcal{O}_{S}} \hat{\mathcal{O}}_{S}(-j)
$$

Proof. This is immediate from Proposition 2.2 .3 by passing to gradeds.

In particular, one sees that

$$
\operatorname{Fil}_{0}\left(R^{i} \pi_{*} \hat{\mathbb{Z}}_{p, X} \otimes_{\hat{\mathbb{Z}}_{p, S}} \hat{\mathcal{O}}_{S}\right)=R^{i} \pi_{*} \mathcal{O}_{X} \otimes_{\mathcal{O}_{S}} \hat{\mathcal{O}}_{S}
$$

This map can be identified.

Proposition 2.2.5. The first filtration step $\mathrm{Fil}_{0}$ of the relative Hodge-Tate filtration is given by the natural map

$$
R^{i} \pi_{*} \mathcal{O}_{X} \otimes_{\mathcal{O}_{S}} \hat{\mathcal{O}}_{S} \rightarrow R^{i} \pi_{*} \hat{\mathcal{O}}_{X} \cong R^{i} \pi_{*} \hat{\mathbb{Z}}_{p, X} \otimes_{\hat{\mathbb{Z}}_{p, S}} \hat{\mathcal{O}}_{S},
$$

which is injective.

We note that in Sch15b], only the first step of the Hodge-Tate filtration was used (for $i=1$ ), and it was defined as the natural map

$$
R^{i} \pi_{*} \mathcal{O}_{X} \otimes_{\mathcal{O}_{S}} \hat{\mathcal{O}}_{S} \rightarrow R^{i} \pi_{*} \hat{\mathcal{O}}_{X}
$$

Proof. We have to identify the image of $\mathbb{M}_{0} \rightarrow \operatorname{gr}^{0} \mathbb{M}$. This can be done after $\otimes_{\mathbb{B}_{\mathrm{dR}, S}^{+}} \mathcal{O} \mathbb{B}_{\mathrm{dR}, S}^{+}$, as this operation preserves gr ${ }^{0}$. Now note that

$$
\mathbb{M}_{0} \otimes_{\mathbb{B}_{\mathrm{dR}, S}^{+}} \mathcal{O} \mathbb{B}_{\mathrm{dR}, S}^{+}=R^{i} \pi_{\mathrm{dR} *} \mathcal{O}_{X} \otimes_{\mathcal{O}_{S}} \mathcal{O} \mathbb{B}_{\mathrm{dR}, S}^{+},
$$

and

$$
\mathbb{M} \otimes_{\mathbb{B}_{\mathrm{dR}, S}^{+}} \mathcal{O} \mathbb{B}_{\mathrm{dR}, S}^{+}=R^{i} \pi_{\mathrm{dR} *} \mathcal{O} \mathbb{B}_{\mathrm{dR}, X}^{+},
$$

by the relative Poincaré lemma. The map $\mathbb{M}_{0} \rightarrow \mathbb{M}$ is induced by the natural inclusion $\mathcal{O}_{X} \rightarrow \mathcal{O B}_{\mathrm{dR}, X}^{+}$, which commutes with the natural connections.

Passing to $\operatorname{gr}^{0}$ on the side of $\mathbb{M}$ replaces the relative de Rham complex of $\mathcal{O B} \mathbb{R}_{\mathrm{dR}, X}^{+}$ with just $\hat{\mathcal{O}}_{X}$, as the differentials sit in positive degrees. We note that the composite $\mathcal{O}_{X} \rightarrow \mathcal{O} \mathbb{B}_{\mathrm{dR}, X}^{+} \rightarrow \hat{\mathcal{O}}_{X}$ is the natural inclusion, as

$$
\hat{\mathcal{O}}_{X}=\operatorname{gr}^{0} \mathcal{O} \mathbb{B}_{\mathrm{dR}, X}^{+}=\left(\mathcal{O}_{X} \otimes_{W(k)} W\left(\hat{\mathcal{O}}_{X^{b}}^{+}\right)\right) /(\operatorname{ker} \theta),
$$

using the map $\theta: \mathcal{O}_{X} \otimes_{W(k)} W\left(\hat{\mathcal{O}}_{X^{b}}^{+}\right) \rightarrow \hat{\mathcal{O}}_{X}$, which is $\mathcal{O}_{X}$-linear. It follows that the map

agrees with the map

$$
\mathbb{M}_{0} \otimes_{\mathbb{B}_{\mathrm{dR}, S}^{+}} \mathcal{O} \mathbb{B}_{\mathrm{dR}, S}^{+} \rightarrow \operatorname{gr}^{0} \mathbb{M}
$$

$$
R^{i} f_{\mathrm{dR} *} \mathcal{O}_{X} \otimes_{\mathcal{O}_{S}} \mathcal{O} \mathbb{B}_{\mathrm{dR}, S}^{+} \rightarrow R^{i} f_{*} \hat{\mathcal{O}}_{X}
$$


which projects $R^{i} f_{\mathrm{dR} *} \mathcal{O}_{X} \rightarrow R^{i} f_{*} \mathcal{O}_{X} \rightarrow R^{i} f_{*} \hat{\mathcal{O}}_{X}$, and then extends $\mathcal{O} \mathbb{B}_{\mathrm{dR}, S^{-}}^{+}$ linearly. Thus, its image is given by the image of $R^{i} f_{*} \mathcal{O}_{X} \otimes_{\mathcal{O}_{S}} \hat{\mathcal{O}}_{S} \rightarrow R^{i} f_{*} \hat{\mathcal{O}}_{X}$. By the identification of the graded pieces of the relative Hodge-Tate filtration, this map has to be injective, giving the result.

\subsection{Hodge cycles and torsors. Let}

$$
(G, X) \hookrightarrow(\tilde{G}, \tilde{X})
$$

be an embedding of Shimura data, as in the previous section, where $\tilde{G}=G S p(V, \psi)$. Let

$$
V^{\otimes}:=\bigoplus_{r, s \in \mathbb{N}} V^{\otimes r} \otimes\left(V^{\vee}\right)^{\otimes s}
$$

By Proposition 3.1 of Del82, the subgroup $G$ of $\tilde{G}$ is the pointwise stabilizer of a finite collection of tensors $\left(s_{\alpha}\right) \subset V^{\otimes}$.

As above, the embedding of Shimura data determines an embedding of Shimura varieties defined over $E$ :

$$
S_{K} \hookrightarrow \tilde{S}_{\tilde{K}} \otimes_{\mathbb{Q}} E
$$

Let $\mathcal{A}$ be the abelian scheme over $S_{K}$ obtained by pulling back the universal abelian scheme over the Siegel moduli space. Let $\pi: \mathcal{A} \rightarrow S_{K}$ be the projection. The first relative Betti homology of $\mathcal{A}$, i.e. the dual of $R^{1} \pi_{*}^{\text {an }} \mathbb{Q}$, defines a local system of $\mathbb{Q}$-vector spaces $\mathcal{V}_{B}$ on $S_{K}(\mathbb{C})$. Since the Betti cohomology of an abelian variety parametrized by $X \times G\left(\mathbb{A}_{f}\right) / K$ gets identified with $V, \mathcal{V}_{B}$ can be identified with the local system of $\mathbb{Q}$-vector spaces over $S_{K}(\mathbb{C})$ given by the $G(\mathbb{Q})$-representation $V$ and the $G(\mathbb{Q})$-torsor

$$
X \times G\left(\mathbb{A}_{f}\right) / K \rightarrow G(\mathbb{Q}) \backslash\left(X \times G\left(\mathbb{A}_{f}\right) / K\right)=S_{K}(\mathbb{C}) .
$$

Corresponding to the $G(\mathbb{Q})$-invariant tensors $\left(s_{\alpha}\right)$, we get global sections $\left(s_{\alpha, B}\right) \subset$ $\mathcal{V}_{B}^{\otimes}$. Moreover, these are Hodge tensors for the Hodge structure on Betti homology, since they are $G$-invariant, and in particular invariant under the action of any $h \in X$.

Lemma 2.3.1. The $G(\mathbb{Q})$-torsor

$$
X \times G\left(\mathbb{A}_{f}\right) / K \rightarrow G(\mathbb{Q}) \backslash\left(X \times G\left(\mathbb{A}_{f}\right) / K\right)=S_{K}(\mathbb{C})
$$

can be identified with the $G(\mathbb{Q})$-torsor sending any $U \subset S_{K}(\mathbb{C})$ to

$$
\left\{\beta: V \times\left. U \cong \mathcal{V}_{B}\right|_{U} \mid \beta\left(s_{\alpha}\right)=s_{\alpha, B}\right\} .
$$

Proof. This follows from the fact that $G \subset \mathrm{GL}(V)$ is the closed subgroup which is the stabilizer of the $s_{\alpha}$.

Now assume that $(G, X) \hookrightarrow\left(\tilde{G}^{\prime}, \tilde{X}^{\prime}\right)$ is a second symplectic embedding, where $\tilde{G}^{\prime}=G S p\left(V^{\prime}, \psi^{\prime}\right)$. Like for any representation of $G$, there is a $G$-invariant idempotent $e \in V^{\otimes}$ such that $V^{\prime}=e V^{\otimes}$. Using $e$, any $G$-invariant tensor $s_{\alpha}^{\prime} \in\left(V^{\prime}\right)^{\otimes}$ can be transferred to a $G$-invariant tensor in $V^{\otimes}$. Moreover, one also has an identification

$$
\mathcal{V}_{B}^{\prime}=e \mathcal{V}_{B}^{\otimes}
$$

compatibly with their natural Hodge structures. We will generally assume that $e$ belongs to the family $s_{\alpha}$, by adjoining it if necessary.

Let $\mathcal{V}_{\mathrm{dR}}:=\left(R^{1} \pi_{\mathrm{dR} *} \mathcal{O}_{\mathcal{A}}\right)^{\vee}$ be the first relative de Rham homology of $\mathcal{A}$. This is a vector bundle over $S_{K}$ equipped with an integrable connection $\nabla$. The base change 
to $\mathbb{C}$ can be defined directly: We have to specify an analytic vector bundle $\mathcal{V}_{\mathrm{dR}, \mathbb{C}}^{\mathrm{C}}$ over $S_{K}(\mathbb{C})$, which corresponds to the algebraic vector bundle $\mathcal{V}_{\mathrm{dR}, \mathbb{C}}$. (Here, we make use of the equivalence of categories between algebraic vector bundles equipped with a flat connection with regular singular points and analytic vector bundles equipped with a flat connection [Del70].) Then the relative de Rham comparison isomorphism over $\mathbb{C}$ gives rise to an isomorphism

$$
\mathcal{V}_{\mathrm{dR}, \mathbb{C}}^{\mathrm{an}} \cong \mathcal{V}_{B} \otimes_{\mathbb{Q}} \mathcal{O}_{S_{K}(\mathbb{C})}
$$

compatible with the connection.

In particular, the global sections $\left(s_{\alpha, B}\right) \subset \mathcal{V}_{B}^{\otimes}$ give rise to horizontal global sections $\left(s_{\alpha, \mathrm{dR}}\right) \subset\left(\mathcal{V}_{\mathrm{dR}, \mathbb{C}}^{\mathrm{an}}\right)^{\otimes}$, which are necessarily algebraic, i.e.

$$
\left(s_{\alpha, \mathrm{dR}}\right) \subset \mathcal{V}_{\mathrm{dR}, \mathbb{C}}^{\otimes} .
$$

The following lemma appears in work of Kisin Kis10, based on Deligne's result that Hodge cycles on abelian varieties are absolute Hodge, Del82.

Lemma 2.3.2. The tensors $s_{\alpha, \mathrm{dR}}$ in $\mathcal{V}_{\mathrm{dR}, \mathbb{C}}^{\otimes}$ are defined over $E$.

Proof. We sketch Kisin's proof here. We work with each connected component of $S_{K}$ individually. Let $x$ be the generic point of one such component, with function field $\kappa$ (containing $E$ ) and choose a complex embedding of its algebraic closure $\bar{\kappa} \hookrightarrow \mathbb{C}$. Let $\mathcal{A}_{x}$ be the corresponding abelian variety over $\kappa$. Let $s_{\alpha, B, x}$ be the fiber of $s_{\alpha, B}$ over $x$. Let $s_{\alpha, \mathrm{dR}, x} \in H_{\mathrm{dR}}^{1}\left(\mathcal{A}_{x}\right)^{\otimes} \otimes_{\kappa} \mathbb{C}$ be the image of $s_{\alpha, B, x}$ under the de Rham comparison isomorphism (this is also the fiber of $s_{\alpha, \mathrm{dR}}$ over $x$.) Let $s_{\alpha, p, x} \in H_{\text {ét }}^{1}\left(\mathcal{A}_{x, \bar{\kappa}}, \mathbb{Q}_{p}\right)^{\otimes}$ be the image of $s_{\alpha, B, x}$ under the comparison between Betti and $p$-adic étale cohomology.

Note that by definition $\left(s_{\alpha, \mathrm{dR}, x}, s_{\alpha, p, x}\right)$ is a Hodge cycle. By Deligne [Del82, it is an absolute Hodge cycle. This means that $s_{\alpha, \mathrm{dR}, x}$ is defined over $\bar{\kappa}$ and it remains to show that the action of $\operatorname{Gal}(\bar{\kappa} / \kappa)$ on it is trivial. For this, it is enough to check that the $\operatorname{Gal}(\bar{\kappa} / \kappa)$-action on $s_{\alpha, p, x}$ is trivial, since a Hodge cycle is determined by either its de Rham or étale component.

For this latter statement, consider the $\tilde{K}_{p}$-torsor over Siegel moduli space given by $\lim _{\tilde{K}_{p}^{\prime}} \tilde{S}_{\tilde{K}^{p} \tilde{K}_{p}^{\prime}}$, where $\tilde{K}_{p}^{\prime}$ runs over open compact subgroups of $\tilde{K}_{p}$. Fixing a $\bar{\kappa}$-point $\tilde{x}$ of this tower above $x$, the $\operatorname{Gal}(\bar{\kappa} / \kappa)$-action on $H_{\text {ét }}^{1}\left(\mathcal{A}_{x, \bar{\kappa}}, \mathbb{Q}_{p}\right)$ is induced by the map $\operatorname{Gal}(\bar{\kappa} / \kappa) \rightarrow \tilde{K}_{p}$ describing the action on $\tilde{x}$. There is an analogous $K_{p}$-torsor over $S_{K}$ defined by $\lim _{K_{p}^{\prime}} S_{K^{p}} K_{p}^{\prime}$. This fits into a commutative, $K_{p^{-}}$ equivariant diagram

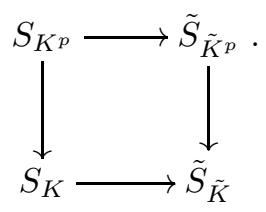

Taking for $\tilde{x}$ a lift to $S_{K^{p}}$, we see that the action of $\operatorname{Gal}(\bar{\kappa} / \kappa)$ on $H_{\text {ét }}^{1}\left(\mathcal{A}_{x, \bar{\kappa}}, \mathbb{Q}_{p}\right)$ factors through a map

$$
\operatorname{Gal}(\bar{\kappa} / \kappa) \rightarrow K_{p} \subset G\left(\mathbb{Q}_{p}\right) .
$$

Since the tensors $s_{\alpha, p, x}$ are $G\left(\mathbb{Q}_{p}\right)$-invariant, the Galois action on these tensors is trivial as well. 
Remark 2.3.3. If $(G, X) \hookrightarrow\left(\tilde{G}^{\prime}, \tilde{X}^{\prime}\right)$ is a second symplectic embedding with $\tilde{G}^{\prime}=$ $G \operatorname{Sp}\left(V^{\prime}, \psi^{\prime}\right)$, and $e \in V^{\otimes}$ is an idempotent with $V^{\prime}=e V^{\otimes}$, as above, then applying Lemma 2.3 .2 to the embedding $G \hookrightarrow G S p\left(V \oplus V^{\prime}, \psi \oplus \psi^{\prime}\right)$, one sees that the isomorphism

is defined over $E$.

$$
\mathcal{V}_{\mathrm{dR}, \mathbb{C}}^{\prime} \cong e_{\mathrm{dR}} \mathcal{V}_{\mathrm{dR}, \mathbb{C}}^{\otimes}
$$

There is also a $\mathbb{Q}_{p}$-local system $\mathcal{V}_{p}$ over $S_{K}$ defined by restricting to $S_{K}$ the first relative $p$-adic étale homology of the family $\mathcal{A}$. There are families of Hodge tensors $\left(s_{\alpha, p}\right) \subset \mathcal{V}_{p}^{\otimes}$ coming from the comparison between Betti and $p$-adic étale homology (over $\mathbb{C}$ ). By the argument in Lemma 2.3.2, the $s_{\alpha, p}$ are also defined over the reflex field $E$.

Choose a cocharacter $\mu$ in the conjugacy class $X$, which is defined over some finite extension $E^{\prime} / E$. We will base change everything to $E^{\prime}$ from now on, but drop $E^{\prime}$ from our notation. Recall that $P_{\mu}^{\text {std }}$ can be identified with the parabolic subgroup of $G$ which stabilizes the descending filtration induced by $\mu$ on a faithful representation $V$ of $G$. We can define a $P_{\mu}^{\text {std }}$-torsor $\mathcal{P}_{\mathrm{dR}}$ over $S_{K}$ as the torsor of frames on the vector bundle $\mathcal{V}_{\mathrm{dR}}$ which respect the Hodge filtration. More precisely, for any $U \subset S_{K}$, we have:

$$
\mathcal{P}_{\mathrm{d} R}(U)=\left\{\beta:\left.\mathcal{V}_{\mathrm{dR}}\right|_{U} \stackrel{\sim}{\rightarrow} V \otimes_{\mathbb{Q}} \mathcal{O}_{U} \mid \beta\left(s_{\alpha, \mathrm{dR}}\right)=s_{\alpha} \otimes 1, \beta\left(\mathrm{Fil}^{\bullet}\right)=\mathrm{Fil}_{\mu}^{\bullet}\right\},
$$

where Fil $\bullet^{\bullet}$ on $\mathcal{V}_{\mathrm{dR}}$ is the Hodge filtration and $\mathrm{Fil}_{\mu}^{\bullet}$ on $V$ is the descending filtration defined by $\mu$. The existence of one such isomorphism $\beta$ follows from the fact that the comparison between Betti and de Rham cohomology respects the Hodge filtrations and matches the Hodge cycles $s_{\alpha}$ with $s_{\alpha, \mathrm{dR}}$.

Lemma 2.3.4. The $P_{\mu}^{\mathrm{std}}$-torsor $\mathcal{P}_{\mathrm{dR}}$ over $S_{K}$ is independent of the choice of symplectic embedding $G \hookrightarrow G S p(V, \psi)$.

Proof. Considering a second symplectic embedding $G \hookrightarrow G S p\left(V^{\prime}, \psi^{\prime}\right)$, there is a $G$ invariant idempotent $e \in V^{\otimes}$ such that $V^{\prime}=e V^{\otimes}$. This determines a Hodge tensor $e_{B}$ in $\mathcal{V}_{B}^{\otimes}$, and by Lemma 2.3.2 a tensor $e_{\mathrm{dR}}$ in $\mathcal{V}_{\mathrm{dR}}^{\otimes}$. This defines an isomorphism of vector bundles $\mathcal{V}_{\mathrm{dR}}^{\prime} \simeq e_{\mathrm{dR}} \mathcal{V}_{\mathrm{dR}}^{\otimes}$ by Remark 2.3.3, which respects all the Hodge tensors $s_{\alpha, \mathrm{dR}}$ and which respects the Hodge filtration on the two vector bundles (because $e_{\mathrm{dR}}$ is a Hodge tensor). This gives a map of $P_{\mu}^{\text {std }}$-torsors $\mathcal{P}_{\mathrm{dR}} \rightarrow \mathcal{P}_{\mathrm{dR}}^{\prime}$ and any such map is an isomorphism.

From the above $P_{\mu}^{\text {std }}$-torsor $\mathcal{P}_{\mathrm{dR}}$ and from the projection $P_{\mu} \rightarrow M_{\mu}$, we get an $M_{\mu}$-torsor $\mathcal{M}_{\mathrm{dR}}$ over $S_{K}$ via pushout:

$$
\mathcal{M}_{\mathrm{dR}}=\mathcal{P}_{\mathrm{dR}} \times_{P_{\mu}^{\mathrm{std}}} M_{\mu} .
$$

Since $\mathcal{P}_{\mathrm{dR}}$ is independent of the choice of symplectic embedding, so is $\mathcal{M}_{\mathrm{dR}}$. This $M_{\mu}$-torsor corresponds to trivializing the graded pieces of the Hodge filtration on $\mathcal{V}_{\mathrm{dR}}$ individually. By the Tannakian formalism, $\mathcal{M}_{\mathrm{dR}}$ is equivalent to a functor from finite-dimensional representations of the Levi subgroup $M_{\mu}$ to vector bundles on $S_{K}$.

Lemma 2.3.5. The $M_{\mu}$-torsor $\mathcal{M}_{\mathrm{dR}}$ encodes the tensor functor

$$
f_{\infty}: \operatorname{Rep} M_{\mu} \rightarrow \text { \{automorphic vector bundles on } S_{K} \text { \} }
$$

in the statement of Theorem 2.1.3. 
Proof. By construction, the tensor functor corresponding to $\mathcal{M}_{\mathrm{dR}}$ factors through the inflation map Rep $M_{\mu} \rightarrow \operatorname{Rep} P_{\mu}^{\text {std }}$.

It remains to see that the functor corresponding to $P_{\mu}^{\text {std }}$ maps a representation of $P_{\mu}^{\text {std }}$ to the associated automorphic vector bundle on $S_{K}$. This is essentially the definition of automorphic vector bundles, as given by [Mil90. For this, note that $\mathcal{P}_{\mathrm{dR}}$ and the map $P_{\mu}^{\text {std }} \rightarrow G$ define by pushout a $G$-torsor $\mathcal{G}_{\mathrm{dR}}$ over $S_{K}$, which parametrizes frames of $\mathcal{V}_{\mathrm{dR}}$ respecting the Hodge tensors $s_{\alpha, \mathrm{dR}}$ (but not necessarily respecting the Hodge filtration). This is what Milne calls the standard principal bundle. Since it was constructed from a $P_{\mu}^{\text {std }}$-torsor, $\mathcal{G}_{\mathrm{dR}}$ is equipped with a canonical map to the flag variety $\mathrm{Fl}_{G, \mu}^{\mathrm{std}} \simeq G / P_{\mu}$. We have a diagram

$$
S_{K} \longleftarrow \mathcal{G}_{\mathrm{dR}} \longrightarrow \mathrm{Fl}_{G, \mu}^{\mathrm{std}} \text {. }
$$

Proposition 3.5 of [Mil90 proves that automorphic vector bundles are obtained by pullback from $\mathrm{Fl}_{G, \mu}$ to $\mathcal{G}_{\mathrm{dR}}$ followed by descent to $S_{K}$. We note that Theorems 4.1 and 4.3 of Mil90] show that the diagram is algebraic and has a model over the reflex field $E$.

We now work with the local system $\mathcal{V}_{p}$ determined by the relative $p$-adic étale cohomology of $\mathcal{A}$. This is a local system of $\mathbb{Q}_{p}$-vector spaces over $S_{K}$. After pulling it back to the adic space $\mathcal{S}_{K}$, we can think of it as a locally free $\hat{\mathbb{Q}}_{p}$-module on $\left(\mathcal{S}_{K}\right)_{\text {proét }}$.

Regard $P_{\mu}$ as a group object in the pro-étale site of $\mathcal{S}_{K}$ by sending $U$ to $P_{\mu}\left(\hat{\mathcal{O}}_{\mathcal{S}_{K}}(U)\right)$; we emphasize that we are using the completed structure sheaf in this definition. We can now define a $P_{\mu}$-quasitorsor $\mathscr{P}_{p}$ on the pro-étale site of $\mathcal{S}_{K}$ from the Hodge-Tate filtration on $\mathcal{V}_{p} \otimes_{\hat{\mathbb{Q}}_{p}} \hat{\mathcal{O}}_{S_{K}}$ as follows. For $U$ in $\left(\mathcal{S}_{K}\right)_{\text {proét }}$, set

$\mathscr{P}_{p}(U)=\left\{\beta:\left.\left.\mathcal{V}_{p} \otimes_{\hat{\mathbb{Q}}_{p}} \hat{\mathcal{O}}_{\mathcal{S}_{K}}\right|_{U} \stackrel{\sim}{\rightarrow} V \otimes_{\mathbb{Q}} \hat{\mathcal{O}}_{\mathcal{S}_{K}}\right|_{U} \mid \beta\left(s_{\alpha, p} \otimes 1\right)=s_{\alpha} \otimes 1, \beta\left(\operatorname{Fil}_{\bullet}\right)=\right.$ Fil $\left._{\bullet}(\mu)\right\}$,

where Fil. on $\mathcal{V}_{p} \otimes \hat{\mathcal{O}}_{S_{K}}$ is the relative Hodge-Tate filtration and Fil. $(\mu)$ is the ascending filtration determined by $\mu$ on $V$.

Lemma 2.3.6. The object $\mathscr{P}_{p}$ over $\mathcal{S}_{K}$ is a $P_{\mu}$-torsor.

Proof. Similarly to $\mathscr{P}_{p}$, one can define a $G$-quasitorsor $\mathscr{G}_{p}$ over the pro-étale site of $\mathcal{S}_{K}$, by removing the condition on filtrations. The latter is the pushout of a $G\left(\hat{\mathbb{Q}}_{p}\right)$ torsor on the pro-étale site of $\mathcal{S}_{K}$ given by looking at isomorphisms between $\mathcal{V}_{p}$ and $V \otimes \mathbb{Q}_{p} \hat{\mathbb{Q}}_{p}$ respecting all tensors. This is a torsor, since, for example, it admits a global section over the perfectoid Shimura variety $\mathcal{S}_{K^{p}}$. In order to prove that $\mathscr{P}_{p}$ is a torsor, we note that the type of the Hodge-Tate filtration on $\mathcal{V}_{p} \otimes_{\hat{\mathbb{Q}}_{p}} \hat{\mathcal{O}}_{\mathcal{S}_{K}}$ is a discrete invariant, so it is constant on each connected component of $\mathcal{S}_{K}$. Therefore, it suffices to check the statement above classical points.

Thus, let $x \in \mathcal{S}_{K}\left(L, \mathcal{O}_{L}\right)$ be a point defined over a finite extension $L$ of $E_{\mathfrak{p}}$ with completed algebraic closure $C$. We may pick a point of $\mathcal{M}_{\mathrm{dR}}(C)$ above $x$, which amounts to trivializing all Hodge cohomology groups (compatibly with the tensors). Then the Hodge-Tate decomposition reads

$$
\mathcal{V}_{p, x} \otimes_{\mathbb{Q}_{p}} C \cong \bigoplus_{j} V_{j} \otimes C(-j) \cong V \otimes C,
$$


where $V=\bigoplus_{j} V_{j}$ is the weight decomposition according to the action of $\mu$, and we are using any fixed choice of $p$-power roots of unity in $C$ in the second isomorphism. Under this isomorphism, the Hodge-Tate filtration on the left-hand side is taken to Fil. $(\mu)$, as desired.

The fact that $s_{\alpha, p}$ can be identified with $s_{\alpha}$ under the Hodge-Tate isomorphism is proved in [Bla94].

As before, this torsor is independent of the choice of symplectic embedding.

Lemma 2.3.7. The $P_{\mu}$-torsor $\mathscr{P}_{p}$ is independent of the choice of symplectic embedding.

Proof. This uses the same idea as the proof of Lemma 2.3.4. Let $(V, \psi)$ be a symplectic embedding of $G$, which defines the $P_{\mu}$-torsor $\mathscr{P}_{p}$. For another symplectic embedding $G \hookrightarrow G S p\left(V^{\prime}, \psi^{\prime}\right)$, we define a $P_{\mu}$-torsor $\mathscr{P}_{p}^{\prime}$ analogously. We can relate the two symplectic embeddings given by $(V, \psi)$ and $\left(V^{\prime}, \psi^{\prime}\right)$ via a $G$-invariant idempotent $e \in V^{\otimes}$, with $p$-adic realization $e_{p} \in \mathcal{V}_{p}^{\otimes}$. The tensor $e_{p}$ defines an isomorphism of vector bundles

$$
\mathcal{V}_{p}^{\prime} \otimes \hat{\mathcal{O}}_{\mathcal{S}_{K}} \simeq e_{p}\left(\mathcal{V}_{p}^{\otimes} \otimes \hat{\mathcal{O}}_{\mathcal{S}_{K}}\right)
$$

which matches the tensors $s_{\alpha, p}^{\prime} \in \mathcal{V}_{p}^{\prime \otimes}$ with tensors in $\mathcal{V}_{p}^{\otimes}$.

Moreover, $e_{p}$ respects the Hodge-Tate filtration on the two vector bundles. Indeed, $e_{p}$ is the image of $e_{\mathrm{dR}}$ under the $p$-adic-de Rham comparison isomorphism. At points of $S_{K}$ corresponding to abelian varieties defined over number fields, this follows from [Bla94. Since both $e_{p}$ and $e_{\mathrm{dR}}$ are horizontal sections, the result extends over all of $\mathcal{S}_{K}$ after checking it at such a point in every connected component of $\mathcal{S}_{K}$. The definition of the relative Hodge-Tate filtration in terms of the $p$-adic-de Rham comparison isomorphisms then ensures that $e_{p}$ respects the Hodge-Tate filtration, and the isomorphism induced by $e_{p}$ gives a map of $P_{\mu}$-torsors $\mathscr{P}_{p} \rightarrow \mathscr{P}_{p}^{\prime}$, which has to be an isomorphism.

The $P_{\mu}$-torsor $\mathscr{P}_{p}$ defines a $G$-torsor $\mathscr{G}_{p}$ by inflation along the map $P_{\mu} \rightarrow G$. For any $U \in\left(\mathcal{S}_{K}\right)_{\text {proét }}$,

$$
\mathscr{G}_{p}(U)=\left\{\beta:\left.\left.\mathcal{V}_{p} \otimes_{\hat{\mathbb{Q}}_{p}} \hat{\mathcal{O}}_{\mathcal{S}_{K}}\right|_{U} \stackrel{\sim}{\rightarrow} V \otimes_{\mathbb{Q}} \hat{\mathcal{O}}_{\mathcal{S}_{K}}\right|_{U} \mid \beta\left(s_{\alpha, p} \otimes 1\right)=s_{\alpha} \otimes 1\right\} .
$$

The perfectoid Shimura variety $\mathcal{S}_{K^{p}}$ can be regarded as a $K_{p}$-torsor in $\left(\mathcal{S}_{K}\right)_{\text {proét }}$. From the moduli description of $\mathcal{S}_{K^{p}}$, we see that $\mathscr{G}_{p}\left(\mathcal{S}_{K^{p}}\right)$ has a canonical section, given by the trivialization of the $p$-adic Tate module of the universal abelian variety $\mathcal{A}$ over $\mathcal{S}_{K^{p}}$, which by definition respects the tensors $\left(s_{\alpha, p}\right)$.

The map $P_{\mu} \rightarrow M_{\mu}$ defines an $M_{\mu}$-torsor $\mathscr{M}_{p}$ by pushout. This can be described as a sheaf on $\left(\mathcal{S}_{K}\right)_{\text {proét }}$ as follows:

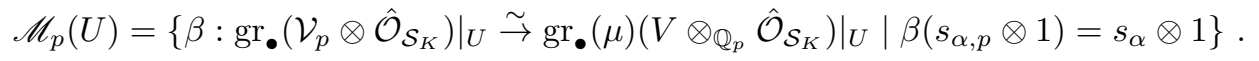

As in the complex case, the existence of $\mathscr{P}_{p}$ determines a map $\mathscr{G}_{p} \rightarrow \mathscr{F} \ell_{G, \mu}$, which is independent of the choice of symplectic embedding $G \hookrightarrow G S p(V, \psi)$ by Lemma 2.3.7. Here, we abuse notation by writing $\mathscr{F} \ell_{G, \mu}$ for the sheaf on $\left(\mathcal{S}_{K}\right)_{\text {proét }}$ sending $U$ to $\mathscr{F} \ell_{G, \mu}(U)$. This and the given section of $\mathscr{G}_{p}\left(\mathcal{S}_{K^{p}}\right)$ define an element of $\mathscr{F} \ell_{G, \mu}\left(\mathcal{S}_{K^{p}}\right)$, i.e. a map of adic spaces

$$
\pi_{H T}: \mathcal{S}_{K^{p}} \rightarrow \mathscr{F} \ell_{G, \mu} .
$$


By functoriality of this construction (for $G$ and for $\tilde{G}:=G S p(V, \psi)$ ), we have the commutative diagram of adic spaces

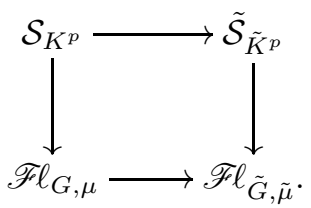

Therefore, the Hodge-Tate period map for $\mathcal{S}_{K^{p}}$ factors through this canonical map $\mathcal{S}_{K^{p}} \rightarrow \mathscr{F} \ell_{G, \mu}$. This proves the first part of Theorem 2.1.3.

The second part of Theorem 2.1.3 will follow from the next lemma and from the comparison isomorphism between de Rham and $p$-adic étale cohomology.

Lemma 2.3.8. The $M_{\mu}$-torsor $\mathscr{M}_{p}$ encodes the tensor functor

$$
f_{p}: \operatorname{Rep} M_{\mu} \rightarrow\left\{G\left(\mathbb{Q}_{p}\right) \text { - equivariant vector bundles on } \mathcal{S}_{K^{p}}\right\}
$$

in the statement of Theorem 2.1.3.

Proof. This is immediate from the definitions.

We now compare the two $M_{\mu}$-torsors, $\mathcal{M}_{\mathrm{dR}}$ and $\mathscr{M}_{p}$. For this, we first consider a $P_{\mu}^{\text {std }}$-torsor $\mathscr{P}_{\mathrm{dR}}$ over $\mathcal{S}_{K}$, which will be the sheaf on $\left(\mathcal{S}_{K}\right)_{\text {proét }}$ defined by

$$
\mathscr{P}_{\mathrm{dR}}(U)=\left\{\beta:\left.\left.\mathcal{V}_{\mathrm{dR}} \otimes_{\mathcal{O}_{\mathcal{S}_{K}}} \hat{\mathcal{O}}_{\mathcal{S}_{K}}\right|_{U} \stackrel{\sim}{\rightarrow} V \otimes_{\mathbb{Q}} \hat{\mathcal{O}}_{\mathcal{S}_{K}}\right|_{U} \mid \beta\left(s_{\alpha, \mathrm{dR}} \otimes 1\right)=s_{\alpha} \otimes 1, \beta\left(\mathrm{Fil}^{\bullet}\right)=\mathrm{Fil}^{\bullet}(\mu)\right\}
$$

where $\mathrm{Fil}^{\bullet}$ is the Hodge-de Rham filtration on $\mathcal{V}_{\mathrm{dR}}$. It is easy to see from the definitions that $\mathscr{P}_{\mathrm{dR}}$ is the pullback of $\mathcal{P}_{\mathrm{dR}}$ from $S_{K}$ (ringed with $\left.\mathcal{O}_{S_{K}}\right)$ to $\left(\mathcal{S}_{K}\right)$ proét (ringed with $\hat{\mathcal{O}}_{\mathcal{S}_{K}}$ ). We can define $\mathscr{M}_{\mathrm{dR}}$ by pushout. This is also a sheaf on $\left(\mathcal{S}_{K}\right)_{\text {proét }}$, parametrizing isomorphisms

$$
\operatorname{gr}^{\bullet}\left(\mathcal{V}_{\mathrm{dR}} \otimes \hat{\mathcal{O}}_{\mathcal{S}_{K}}\right) \stackrel{\sim}{\rightarrow} \operatorname{gr}^{\bullet}(\mu)\left(V \otimes \hat{\mathcal{O}}_{\mathcal{S}_{K}}\right)
$$

which map the tensors $s_{\alpha, \mathrm{dR}}$ to $s_{\alpha}$. Again, $\mathscr{M}_{\mathrm{dR}}$ is the pullback of $\mathcal{M}_{\mathrm{dR}}$ from $S_{K}$ to $\left(\mathcal{S}_{K}\right)_{\text {proét }}$.

Proposition 2.3.9. There is a canonical isomorphism $\mathscr{M}_{\mathrm{dR}} \cong \mathscr{M}_{p}$ of $M_{\mu}$-torsors on $\left(\mathcal{S}_{K}\right)_{\text {proét }}$, independent of the choice of symplectic embedding.

Proof. The determinant representation $G S p(V, \psi) \rightarrow \mathbb{G}_{m}$ gives rise geometrically to the Tate motive, and is independent of the choice of symplectic embedding. Using this, both torsors map to the torsor of trivializations $\hat{\mathcal{O}}_{\mathcal{S}_{K}}(1) \cong \hat{\mathcal{O}}_{\mathcal{S}_{K}}$. Now, for any $j \in \mathbb{Z}$, there is the isomorphism

$$
\operatorname{gr}^{j}\left(\mathcal{V}_{\mathrm{dR}} \otimes_{\mathcal{O}_{\mathcal{S}_{K}}} \hat{\mathcal{O}}_{\mathcal{S}_{K}}\right) \stackrel{\sim}{\rightarrow} \operatorname{gr}_{j}\left(\mathcal{V}_{p} \otimes_{\hat{\mathbb{Q}}_{p}} \hat{\mathcal{O}}_{\mathcal{S}_{K}}\right)(j)
$$

coming from the relative $p$-adic-de Rham comparison isomorphism, Corollary 2.2.4 One gets a similar comparison for $\mathcal{V}_{\mathrm{dR}}^{\otimes}$ and $\mathcal{V}_{p}^{\otimes}$, and we know by Bla94 that all tensors $s_{\alpha, \mathrm{dR}}$ resp. $s_{\alpha, p}$ are matched at points defined over number fields, and thus globally.

Using these isomorphisms as well as the trivialization $\hat{\mathcal{O}}_{\mathcal{S}_{K}}(1) \cong \hat{\mathcal{O}}_{\mathcal{S}_{K}}$, one writes down the isomorphism $\mathscr{M}_{\mathrm{dR}} \cong \mathscr{M}_{p}$. To check that it is independent of the choice of symplectic embedding, one argues as before. 
As mentioned above, Proposition 2.3.9 implies the second part of Theorem 2.1.3. once we use the Tannakian formalism in Lemmas 2.3.5 and 2.3.8 to reinterpret $\mathscr{M}_{\mathrm{dR}}$ and $\mathscr{M}_{p}$ as tensor functors

$$
\operatorname{Rep} M_{\mu} \rightarrow\left\{G\left(\mathbb{Q}_{p}\right) \text { - equivariant vector bundles on } \mathcal{S}_{K^{p}}\right\} \text {. }
$$




\section{The Newton stratification on the flag VARIETy}

We start with some motivation. Assume that the Shimura varieties $\mathcal{S}_{K}$ are of Hodge type and that $K=K^{p} K_{p} \subset G\left(\mathbb{A}_{f}\right)$ is a compact open subgroup such that $K_{p}$ is hyperspecial. This means that $G$ extends to a reductive group over $\mathbb{Z}_{p}$ and that $K_{p}=G\left(\mathbb{Z}_{p}\right)$. Then (at least if $p>2$ ) the Shimura variety $\mathcal{S}_{K}$ admits an integral model $\mathscr{S}_{K}$ by Kis10. Moreover, as in Section 1.4 of Kis, we can define a Newton stratification on the special fiber of $\mathscr{S}_{K}$, in terms of the Kottwitz set $B\left(G, \mu^{-1}\right)$ (whose definition we recall below). Pulling this stratification back along the continuous specialization map, we get a stratification on $\mathcal{S}_{K}$, which in turn can be pulled back to the perfectoid Shimura variety to get a Newton stratification $\mathcal{S}_{K^{p}}=\bigsqcup_{b \in B\left(G, \mu^{-1}\right)} \mathcal{S}_{K^{p}}^{b}$. There is a unique closed stratum, corresponding to the basic locus and a unique open stratum, corresponding to the $\mu$-ordinary locus.

Our goal in this section is to define a stratification on the flag variety

$$
\mathscr{F} \ell_{G, \mu}=\bigsqcup_{b \in B\left(G, \mu^{-1}\right)} \mathscr{F} \ell_{G, \mu}^{b},
$$

such that the following properties are satisfied:

(1) On points of rank one,

$$
\mathcal{S}_{K^{p}}^{b}=\pi_{H T}^{-1}\left(\mathscr{F} \ell_{G, \mu}^{b}\right) .
$$

(2) All $\mathscr{F} \ell_{G, \mu}^{b}$ are locally closed subspaces of the adic space $\mathscr{F} \ell_{G, \mu}$, in the topological sense.

(3) The basic stratum is open, and the $\mu$-ordinary stratum is closed.

We will define this stratification independently of the one on the Shimura variety, using relative versions of the Fargues-Fontaine curve [FF14] and a classification result for vector bundles with $G$-structure over this curve, due to Fargues, Far15a. We will reinterpret vector bundles over the curve as $\varphi$-modules over the Robba ring, à la Kedlaya-Liu [KL15], and use their results to conclude that the strata we define are locally closed. In Section 4.3 , we will see that this is compatible with the stratification pulled back from the special fiber, in the sense described above, for compact Shimura varieties of PEL type.

Throughout this section, our notation will be purely local, so fix a prime $p$ and a connected reductive group $G$ over $\mathbb{Q}_{p}$. Moreover, we fix a conjugacy class of cocharacters $\mu: \mathbb{G}_{m} \rightarrow G_{\overline{\mathbb{Q}}_{p}}$, defined over the reflex field $E / \mathbb{Q}_{p}$. Often, we will assume that $\mu$ is minuscule, meaning that in the induced action on the Lie algebra of $G$, only the weights $-1,0$ and 1 appear. However, for the moment, $\mu$ is allowed to be arbitrary.

3.1. Background on isocrystals with $G$-structure. We recall here the definition of the sets $B(G)$ and $B(G, \mu)$, originally due to Kottwitz [Kot85. We start with $B(G)$. Let $L:=W\left(\overline{\mathbb{F}}_{p}\right)[1 / p]$. Let $\sigma$ be the automorphism of $L$ induced by the $p$ th power Frobenius on $\overline{\mathbb{F}}_{p}$. There is an action of $G(L)$ on itself by $\sigma$-conjugation, defined by $g \mapsto h g \sigma(h)^{-1}$ for $g, h \in G(L)$. Then $B(G)$ is defined to be the set of $\sigma$-conjugacy classes of elements $b \in G(L)$. (We note that instead of working with $\overline{\mathbb{F}}_{p}$ here, we could work with any algebraically closed field of characteristic $p$, as Kottwitz shows that the definition is independent of this choice.)

One can reinterpret this definition in terms of isocrystals with $G$-structure. Recall the following definition. 
Definition 3.1.1. An isocrystal over $\overline{\mathbb{F}}_{p}$ is a pair $(V, \phi)$ consisting of a finitedimensional L-vector space and a $\sigma$-linear automorphism $\phi$ of $V$. The height of an isocrystal $(V, \phi)$ is the dimension of $V$ over $L$.

An isocrystal with $G$-structure is an exact tensor functor

$$
\operatorname{Rep}_{\mathbb{Q}_{p}} G \rightarrow\left\{\text { Isocrystals } / \overline{\mathbb{F}}_{p}\right\} .
$$

For $G=G L_{n} / \mathbb{Q}_{p}$, the set $B(G)$ is in bijection with the set of isomorphism classes of isocrystals of height $n$ over $\overline{\mathbb{F}}_{p}$ via $b \mapsto\left(L^{n}, b \sigma\right)$. For general $G$, this extends to a bijection between $B(G)$ and isomorphism classes of isocrystals with $G$-structure.

The Dieudonné-Manin classification shows that $B\left(G L_{n}\right)$ is in bijection with a corresponding set of Newton polygons, via the slope decomposition of the isocrystals. More precisely, any isocrystal $(V, \phi)$ over $\overline{\mathbb{F}}_{p}$ is isomorphic to a unique isocrystal of the form

$$
V \cong \bigoplus_{\lambda=s / r \in \mathbb{Q}} V_{\lambda}^{\oplus n_{\lambda}}
$$

where $\lambda=s / r$ runs through rational numbers written in primitive form with $r>0$, the $n_{\lambda}$ are nonnegative integers, almost all zero, and

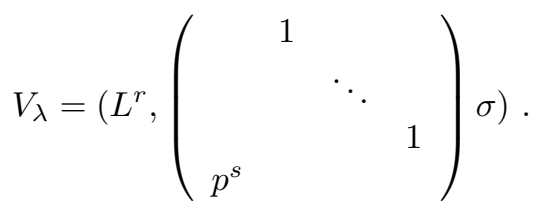

The subspaces $V_{\lambda}^{\oplus n_{\lambda}} \subset V$ are uniquely determined, and referred to as the subspace of slope $\lambda$.

For a general reductive group $G$, an element $b \in B(G)$ is determined by a version of the Newton polygon, and an additional finite datum encoded in the Kottwitz invariant. In the following, fix a splitting of $G_{\overline{\mathbb{Q}}_{p}}$ and in particular a maximal torus $T \subset G_{\overline{\mathbb{Q}}_{p}}$, and let $X_{*}(G):=X_{*}(T)$ be the corresponding cocharacter lattice, which comes with a dominant chamber.

Let us first recall the Newton map

$$
\nu: B(G) \rightarrow\left(X_{*}(G) \otimes \mathbb{Q}\right)_{\text {dom }}^{\Gamma} .
$$

Here, $\Gamma:=\operatorname{Gal}\left(\overline{\mathbb{Q}}_{p} / \mathbb{Q}_{p}\right)$ is the absolute Galois group of $\mathbb{Q}_{p}$, and $\left(X_{*}(G) \otimes \mathbb{Q}\right)_{\text {dom }}$ is the set of dominant rational cocharacters. If we let $\mathbb{D}$ be the (pro-)algebraic torus with character group $\mathbb{Q}$, the latter set can be identified with the set of conjugacy classes of $\operatorname{Hom}\left(\mathbb{D}_{\overline{\mathbb{Q}}_{p}}, G_{\overline{\mathbb{Q}}_{p}}\right)$, on which $\Gamma$ acts naturally.

To construct the Newton map, Kottwitz assigns to any $b \in G(L)$ a slope homomorphism $\nu_{b} \in \operatorname{Hom}\left(\mathbb{D}_{L}, G_{L}\right)$. In the case of $G=G L_{n}$, this gives the slope decomposition of the corresponding isocrystal; in general, it is defined by the Tannakian formalism. Changing $b$ by a $\sigma$-conjugate does not change the conjugacy class of $\nu_{b}$, and (thus) this conjugacy class is invariant under $\sigma$.

However, the Newton map is not, in general, injective. In fact, $\nu_{b}$ is trivial if and only if $b$ is in the image of the natural injection $H^{1}\left(\mathbb{Q}_{p}, G\right) \hookrightarrow B(G)$. Here, one can identify the Galois cohomology group $H^{1}\left(\mathbb{Q}_{p}, G\right)$ with the isomorphism classes of exact tensor functors

$$
\operatorname{Rep}_{\mathbb{Q}_{p}} G \rightarrow\left\{\mathbb{Q}_{p} \text {-vector spaces }\right\}
$$


Such tensor functors embed fully faithfully into the category of isocrystals with $G$ structure, via sending a $\mathbb{Q}_{p}$-vector space $W$ to $W \otimes_{\mathbb{Q}_{p}} L$ with the induced Frobenius from $L$.

For this reason, Kottwitz also constructs a map

$$
\kappa: B(G) \rightarrow \pi_{1}\left(G_{\overline{\mathbb{Q}}_{p}}\right)_{\Gamma} .
$$

For $G=G L_{n}$, this map is defined by $b \mapsto \kappa(b)=\operatorname{val}_{p}(\operatorname{det} b) \in \mathbb{Z}$. In general, there is a unique natural transformation $B() \rightarrow \pi_{1}()_{\Gamma}$ of set-valued functors on the category of connected reductive groups over $\mathbb{Q}_{p}$ with this property. (Kottwitz defines his map in terms of the center of the Langlands dual group. See Section 1.13 of RR96 for more on the definition using the algebraic fundamental group.) Again, we abbreviate $\pi_{1}(G)=\pi_{1}\left(G_{\overline{\mathbb{Q}}_{p}}\right)$. Moreover, according to Theorem 1.15 of [RR96], the natural transformation $B() \rightarrow \pi_{1}()_{\Gamma}$ fits into a commutative diagram

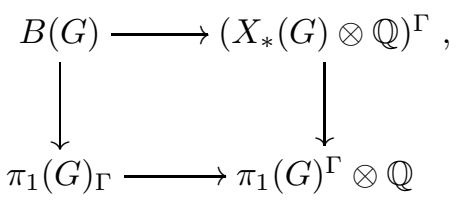

where the lower horizontal arrow is given by averaging over all Galois conjugates. Then Kottwitz proves that

$$
(\nu, \kappa): B(G) \rightarrow\left(X_{*}(G) \otimes \mathbb{Q}\right)_{\mathrm{dom}}^{\Gamma} \times \pi_{1}(G)_{\Gamma}
$$

is injective.

The set $\left(X_{*}(G) \otimes \mathbb{Q}\right)_{\text {dom }}^{\Gamma}$ admits a partial ordering. Under this ordering, we say that $\nu \preceq \nu^{\prime}$ if $\nu^{\prime}-\nu$ is a non-negative $\mathbb{Q}$-linear combination of positive coroots. This defines a partial ordering on $B(G)$, where we say $b \preceq b^{\prime}$ if $\nu_{b} \preceq \nu_{b^{\prime}}$ and $\kappa(b)=\kappa\left(b^{\prime}\right)$.

Now, recall that we have fixed a conjugacy class of cocharacters $\mu: \mathbb{G}_{m} \rightarrow G_{\overline{\mathbb{Q}}_{p}}$. The set of conjugacy classes of cocharacters of $G_{\overline{\mathbb{Q}}_{p}}$ is in bijection with the set $X_{*}(G)_{\text {dom. }}$. There is a natural map $X_{*}(G)_{\text {dom }} \rightarrow\left(X_{*}(G) \otimes \mathbb{Q}\right)_{\text {dom }}^{\Gamma}$ given by averaging over all Galois conjugates:

$$
\bar{\mu}=\frac{1}{\left[E^{\prime}: \mathbb{Q}_{p}\right]} \sum_{\gamma \in \operatorname{Gal}\left(E^{\prime} / \mathbb{Q}_{p}\right)} \gamma(\mu)
$$

for $E^{\prime}$ large enough. Let $\mu^{b}$ be the image of $\mu$ in $\pi_{1}(G)_{\Gamma}$.

Definition 3.1.2. The subset $B(G, \mu) \subset B(G)$ of $\mu$-admissible elements is the subset of elements $b$ for which $\nu_{b} \preceq \bar{\mu}$ and $\kappa(b)=\mu^{b}$.

In fact, we will really be interested in $B\left(G, \mu^{-1}\right)$, where $\mu^{-1}$ denotes a dominant representative of the inverse of $\mu$.

3.2. The Fargues-Fontaine curve. The goal of this subsection is to define the (adic) Fargues-Fontaine curve, and discuss some of its properties. For this, we start with some background on the curve as in [FF14] and Wei14, and then compare with constructions of Kedlaya and Liu [KL15.

Let $F$ be a complete algebraically closed nonarchimedean field of characteristic p, e.g. $F=\widehat{\widehat{\mathbb{F}_{p}((t))}}$. Let $\mathcal{O}_{F} \subset F$ be its ring of integers, i.e. the subring of powerbounded elements. Fix $\varpi \in F$ with $0<|\varpi|<1$; different choices will give 
rise to the same objects. First, we define the Fargues-Fontaine curve as an adic space. Let

$$
\mathcal{Y}_{(0, \infty)}=\operatorname{Spa}\left(W\left(\mathcal{O}_{F}\right), W\left(\mathcal{O}_{F}\right)\right) \backslash(p[\varpi]=0),
$$

where $W\left(\mathcal{O}_{F}\right)$ is endowed with the $(p,[\varpi])$-adic topology. As in Wei14, this space admits a natural continuous map

$$
\alpha: \mathcal{Y}_{(0, \infty)} \rightarrow(0, \infty)
$$

sending any point $x \in \mathcal{Y}_{(0, \infty)}$ to

$$
\alpha(x)=\frac{\log |[\varpi](\tilde{x})|}{\log |p(\tilde{x})|} \in(0, \infty),
$$

where $\tilde{x}$ is the maximal generalization of $x$, which corresponds to a continuous rank1-valuation on $W\left(\mathcal{O}_{F}\right)$ taking nonzero values on $[\varpi]$ and $p$. For any interval $I \subset$ $(0, \infty)$, we let $\mathcal{Y}_{I} \subset \mathcal{Y}_{(0, \infty)}$ be the interior of $\alpha^{-1}(I)$. In the following proposition, we use some terminology from [SW13].

Proposition 3.2.1. For any closed interval $I=[s, r] \subset(0, \infty)$ with $r, s \in \mathbb{Q}$, the space

$$
\mathcal{Y}_{I}=\operatorname{Spa}\left(\mathcal{R}_{F}^{[s, r]}, \mathcal{R}_{F}^{[s, r],+}\right)
$$

is a sheafy affinoid adic space, where $\mathcal{R}_{F}^{[s, r],+}$ is the p-adic completion of the integral closure of

$$
W\left(\mathcal{O}_{F}\right)\left[\frac{p}{\left[\varpi^{1 / r}\right]}, \frac{\left[\varpi^{1 / s}\right]}{p}\right]
$$

inside $W\left(\mathcal{O}_{F}\right)\left[\frac{p}{\left[\varpi^{1 / r}\right]}, \frac{\left[\varpi^{1 / s}\right]}{p}\right][1 / p]$, and $\mathcal{R}_{F}^{[s, r]}=\mathcal{R}_{F}^{[s, r],+}[1 / p]$. More precisely, $\mathcal{R}_{F}^{[s, r]}$ is preperfectoid in the sense that $\mathcal{R}_{F}^{[s, r]} \hat{\otimes}_{\mathbb{Q}_{p}} K$ is a perfectoid $K$-algebra for any perfectoid field $K / \mathbb{Q}_{p}$.

In particular, $\mathcal{Y}_{(0, \infty)}$ is an honest adic space.

Proof. The identification

$$
\mathcal{Y}_{I}=\operatorname{Spa}\left(\mathcal{R}_{F}^{[s, r]}, \mathcal{R}_{F}^{[s, r],+}\right)
$$

follows from the definitions. By [KL15, Theorem 3.7.4], it is enough to show that $\mathcal{R}_{F}^{[s, r]}$ is preperfectoid, for which cf. [KL15, Theorem 5.3.9]. One can also argue as follows. Let $K / \mathbb{Q}_{p}$ be any perfectoid field. We can consider the auxiliary space $\mathcal{Z}=\operatorname{Spa}\left(W\left(\mathcal{O}_{F}\right)[1 / p], W\left(\mathcal{O}_{F}\right)\right)$, where we endow $W\left(\mathcal{O}_{F}\right)$ with the $p$-adic topology. As on $\mathcal{Y}_{(0, \infty)}, p$ is topologically nilpotent, one gets a map $\mathcal{Y}_{(0, \infty)} \rightarrow \mathcal{Z}$, which is an open embedding, and one can thus consider $\mathcal{Y}_{I}$ as a rational subset of $\mathcal{Z}$. As the base change of $\mathcal{Z}$ to $K$ is perfectoid, or more precisely $W\left(\mathcal{O}_{F}\right) \hat{\otimes}_{\mathbb{Z}_{p}} K$ is a perfectoid $K$-algebra, and the property of being a perfectoid $K$-algebra passes to rational subsets, one finds that also $\mathcal{R}_{F}^{[s, r]} \hat{\otimes}_{\mathbb{Q}_{p}} K$ is a perfectoid $K$-algebra.

The space $\mathcal{Y}_{(0, \infty)}$ has an action of $\varphi$, defined by taking the lift of the Frobenius on $\mathcal{O}_{F}$. This $\varphi$-action is properly discontinuous, as can be seen by observing that $\alpha$ is equivariant with respect to the $\varphi$-action if one lets $\varphi$ act through multiplication by $p$ on $(0, \infty)$. Therefore, the following definition is sensible.

Definition 3.2.2. The adic Fargues-Fontaine curve is given by $\mathcal{X}_{F}=\mathcal{Y}_{(0, \infty)} / \varphi^{\mathbb{Z}}$. 
After defining the scheme version of the curve, we will discuss more precisely in which sense this is a curve.

Often, we will be in the situation where we start with a complete algebraically closed nonarchimedean field $C$ over $\mathbb{Q}_{p}$, and take $F=C^{b}$, the tilt of $C$. In that case, there is a natural map $\theta: W\left(\mathcal{O}_{F}\right) \rightarrow \mathcal{O}_{C}$, which induces a natural $\left(C, \mathcal{O}_{C}\right)$ point of $\mathcal{Y}_{(0, \infty)}$, and thus of $\mathcal{X}_{F}$, which we denote by $\infty \in \mathcal{X}_{F}\left(C, \mathcal{O}_{C}\right)$. In fact, $\infty$ is a closed point of $\mathcal{X}_{F}$ with residue field $C$. We will denote the inclusion

$$
i_{\infty}: \operatorname{Spa}\left(C, \mathcal{O}_{C}\right) \rightarrow \mathcal{X}_{F}
$$

The completed local ring of $\mathcal{X}_{F}$ at $\infty$ can be identified with the ring of periods $B_{\mathrm{dR}, C}^{+}$, which is the $\operatorname{ker} \theta$-adic completion of $W\left(\mathcal{O}_{F}\right)[1 / p]$, cf. also Definition 2.2.1 Note that $B_{\mathrm{dR}, C}^{+}$is a complete discrete valuation ring, as expected for the completed local ring of a curve.

There is a close relationship between vector bundles on $\mathcal{X}_{F}$ and isocrystals. Recall that $L$ was defined as $W\left(\overline{\mathbb{F}}_{p}\right)[1 / p]$. A choice of an embedding $\overline{\mathbb{F}}_{p} \rightarrow \mathcal{O}_{F}$ gives a structure map $\mathcal{Y}_{(0, \infty)} \rightarrow \operatorname{Spa}\left(L, \mathcal{O}_{L}\right)$. If $\left(V, \varphi_{V}\right)$ is an isocrystal, one can thus pull it back to a vector bundle on $\mathcal{Y}_{(0, \infty)}$ with a $\varphi$-linear automorphism; by descent, this gives a vector bundle on $\mathcal{X}_{F}$. We denote the resulting functor by $V \mapsto \mathcal{E}(V)$.

Theorem 3.2.3 ([FF14]). The above composition of functors induces a bijection between isomorphism classes of isocrystals, and isomorphism classes of vector bundles on $\mathcal{X}_{F}$.

Remark 3.2.4. In fact, Fargues-Fontaine prove this result for the scheme version of their curve, which we introduce below. However, by a GAGA result proved in [KL15] and [Far15b], this is equivalent to the stated result for the adic curve.

It is important to note that this functor from isocrystals to vector bundles is not an equivalence of categories; there are nonzero maps between vector bundles of different slope, in general.

To define a scheme version of the curve, we define a natural line bundle $\mathcal{O}_{\mathcal{X}_{F}}(1)$ on $\mathcal{X}_{F}$, which we regard as ample.

Definition 3.2.5. For any $d \in \mathbb{Z}$, let $\mathcal{O}_{\mathcal{X}_{F}}(d)$ be the line bundle corresponding to the isocrystal $\left(L, p^{-d} \sigma\right)$.

Remark 3.2.6. This construction induces a map $\mathbb{Z} \rightarrow$ Pic $\mathcal{X}_{F}$. It follows from Theorem 3.2 .3 that this is an isomorphism. Using this identification, one can define the degree of any vector bundle on $\mathcal{X}_{F}$ by looking at the determinant line bundle. This gives rise to a notion of slopes of vector bundles, and a Harder-Narasimhan filtration. We warn the reader that if an isocrystal $V$ is sent to the vector bundle $\mathcal{E}(V)$, then the slopes of $V$ and $\mathcal{E}(V)$ differ by a sign.

Now we define a scheme

$$
X_{F}=\operatorname{Proj}\left(\oplus_{d \geq 0} H^{0}\left(\mathcal{X}_{F}, \mathcal{O}_{\mathcal{X}_{F}}(d)\right)\right) .
$$

There is a natural map of locally ringed topological spaces $\mathcal{X}_{F} \rightarrow X_{F}$. In particular, there is a natural functor from vector bundles on $X_{F}$ to vector bundles on $\mathcal{X}_{F}$. This functor is an equivalence of categories, cf. KL15] and Far15b. The following theorem summarizes some of the properties of $X_{F}$.

Theorem 3.2.7 ([FF14]). The scheme $X_{F}$ is a regular, noetherian scheme of Krull dimension 1 with field of constants $\mathbb{Q}_{p}$. All residue fields of $X_{F}$ at closed points 
are algebraically closed complete extensions $C$ of $\mathbb{Q}_{p}$ with $C^{b} \cong F$. For any closed point $x \in X_{F}, X_{F} \backslash\{x\}$ is the spectrum of a principal ideal domain.

Fargues, Far15a, has recently extended the classification of vector bundles to a classification of $G$-bundles for any reductive group $G$ over $\mathbb{Q}_{p}$. As it is technically easiest for us to do so, we define $G$-bundles on $X_{F}$ (or $\mathcal{X}_{F}$ ) using the Tannakian perspective.

Definition 3.2.8. A G-bundle on $X_{F}\left(\right.$ or $\left.\mathcal{X}_{F}\right)$ is an exact tensor functor

$$
\operatorname{Rep}_{\mathbb{Q}_{p}} G \rightarrow \operatorname{Bun}_{X_{F}} \cong \operatorname{Bun}_{\mathcal{X}_{F}} .
$$

Using the functor from isocrystals over $\overline{\mathbb{F}}_{p}$ to vector bundles on the FarguesFontaine curve, we get a natural functor from isocrystals with $G$-structure to $G$ bundles on $X_{F}$. We denote this functor by $b \mapsto \mathcal{E}_{b}$.

Theorem 3.2.9 (Far15a $)$. The functor from isocrystals with $G$-structure to $G$ bundles on $X_{F}$ induces a bijection on isomorphism classes.

In other words, any $G$-bundle on $X_{F}$ is isomorphic to $\mathcal{E}_{b}$ for a unique $b \in B(G)$.

Next, we discuss the relationship between vector bundles on the Fargues-Fontaine curve and $\varphi$-modules over the Robba ring. The Robba ring is the ring of functions defined on a small unspecified annulus $\mathcal{Y}_{(0, r)}$ :

Definition 3.2.10. The Robba ring is the direct limit

$$
\tilde{\mathcal{R}}_{F}=\underset{r}{\lim } H^{0}\left(\mathcal{Y}_{(0, r]}, \mathcal{O}_{\mathcal{Y}_{(0, r]}}\right) \text {. }
$$

One can make this more explicit, cf. [KL15, Definition 4.2.2]. The space of global sections $\tilde{\mathcal{R}}_{F}^{r}=H^{0}\left(\mathcal{Y}_{(0, r]}, \mathcal{O}_{\mathcal{Y}}\right)$ can be identified with the inverse limit of the Banach algebras $\tilde{\mathcal{R}}_{F}^{[s, r]}$ as $s$ runs over $(0, r]$, and thus acquires a structure of Fréchet algebra. Let

$$
W\left(\mathcal{O}_{F}\right)\left\langle\frac{p}{[\varpi]^{1 / r}}\right\rangle=\left\{\sum_{n \geq 0}\left[c_{n}\right] p^{n} \mid c_{n} \in \varpi^{-n / r} \mathcal{O}_{F}, c_{n} \varpi^{n / r} \rightarrow 0\right\} .
$$

Then $\tilde{\mathcal{R}}_{F}^{r}$ can also be described as the Fréchet completion of

$$
W\left(\mathcal{O}_{F}\right)\left\langle\frac{p}{[\varpi]^{1 / r}}\right\rangle\left[\frac{1}{p}\right]=\left\{\sum_{n>-\infty}\left[c_{n}\right] p^{n} \mid c_{n} \in F, c_{n} \varpi^{n / r} \rightarrow 0\right\}
$$

along the norms $\max _{n}\left\{\left|c_{n} \varpi^{n / s}\right|\right\}$ for $s \in(0, r]$. When $r^{\prime}<r$, there is a natural inclusion map $\tilde{\mathcal{R}}_{F}^{r} \hookrightarrow \tilde{\mathcal{R}}_{F}^{r^{\prime}}$ coming from restriction of global sections. The $\varphi$-action on $\mathcal{Y}_{(0, \infty)}$ sends $\mathcal{Y}_{[s, r]}$ isomorphically to $\mathcal{Y}_{[p s, p r]}$ and $\mathcal{Y}_{(0, r]}$ isomorphically to $\mathcal{Y}_{(0, p r]}$. Therefore, $\varphi$ induces isomorphisms $\tilde{\mathcal{R}}_{F}^{[s, r]} \stackrel{\sim}{\rightarrow} \tilde{\mathcal{R}}_{F}^{[s / p, r / p]}$ and $\tilde{\mathcal{R}}_{F}^{r} \stackrel{\sim}{\rightarrow} \tilde{\mathcal{R}}_{F}^{r / p}$, and thus an automorphism of $\tilde{\mathcal{R}}_{F}$.

We note that the Robba ring is the ring of functions defined on some small punctured disc of unspecified radius around the point $\operatorname{Spa}\left(F, \mathcal{O}_{F}\right)$ of $\operatorname{Spa}\left(W\left(\mathcal{O}_{F}\right), W\left(\mathcal{O}_{F}\right)\right)$.

Definition 3.2.11. A $\varphi$-module over $\tilde{\mathcal{R}}_{F}$ is a finite projective $\tilde{\mathcal{R}}_{F}$-module $M$ equipped with a $\varphi$-linear automorphism.

Remark 3.2.12. As $\tilde{\mathcal{R}}_{F}$ is a Bézout domain, cf. [KL15, Lemma 4.2.6], any $\varphi$-module $M$ is finite free as $\tilde{\mathcal{R}}_{F}$-module. 
Theorem 3.2.13 ([KL15, Theorem 6.3.12]). There is an equivalence of categories $\left\{\right.$ Vector bundles on $\left.\mathcal{X}_{F}\right\} \simeq\left\{\varphi\right.$-modules over $\left.\tilde{\mathcal{R}}_{F}\right\}$.

The proof is based on the observation that any $\varphi$-module over $\tilde{\mathcal{R}}_{F}$ is defined over $\tilde{\mathcal{R}}_{F}^{r}$ for $r$ small enough. This can be turned into a $\varphi$-module over $\mathcal{Y}_{(0, r]}$, and then be spread to a $\varphi$-module over all of $\mathcal{Y}_{(0, \infty)}$ via pullback under Frobenius. By descent, this gives a vector bundle over $\mathcal{X}_{F}$.

3.3. The relative Fargues-Fontaine curve. In this subsection, we extend the constructions to the relative setting. Here, our basic input will be a perfectoid affinoid algebra $\left(R, R^{+}\right)$of characteristic $p 10$ Let $\varpi$ be a pseudouniformizer of $R$. Define

$$
\mathcal{Y}_{(0, \infty)}\left(R, R^{+}\right)=\operatorname{Spa}\left(W\left(R^{+}\right), W\left(R^{+}\right)\right) \backslash(p[\varpi]=0) .
$$

Many constructions carry over to this relative situation. In particular, there is still a continuous map

$$
\alpha: \mathcal{Y}_{(0, \infty)}\left(R, R^{+}\right) \rightarrow(0, \infty)
$$

defined in the same way. Again, we let $\mathcal{Y}_{I}\left(R, R^{+}\right) \subset \mathcal{Y}_{(0, \infty)}\left(R, R^{+}\right)$denote the interior of the preimage $\alpha^{-1}(I)$, for any interval $I \subset(0, \infty)$. Proposition 3.2.1 extends to the relative setting.

Proposition 3.3.1. For any closed interval $I=[s, r] \subset(0, \infty)$ with $r, s \in \mathbb{Q}$, the space

$$
\mathcal{Y}_{I}\left(R, R^{+}\right)=\operatorname{Spa}\left(\mathcal{R}_{R}^{[s, r]}, \mathcal{R}_{R, R^{+}}^{[s, r],+}\right)
$$

is a sheafy affinoid adic space, where $\mathcal{R}_{R, R^{+}}^{[s, r],+}$ is the p-adic completion of the integral closure of

$$
W\left(R^{+}\right)\left[\frac{p}{\left[\varpi^{1 / r}\right]}, \frac{\left[\varpi^{1 / s}\right]}{p}\right]
$$

inside $W\left(R^{+}\right)\left[\frac{p}{\left[\varpi^{1 / r}\right]}, \frac{\left[\varpi^{1 / s}\right]}{p}\right][1 / p]$, and $\mathcal{R}_{R}^{[s, r]}=\mathcal{R}_{R, R^{+}}^{[s, r],+}[1 / p]$ 11] More precisely, $\mathcal{R}_{R}^{[s, r]}$ is preperfectoid in the sense that $\mathcal{R}_{R}^{[s, r]} \hat{\otimes}_{\mathbb{Q}_{p}} K$ is a perfectoid $K$-algebra for any perfectoid field $K / \mathbb{Q}_{p}$.

In particular, $\mathcal{Y}_{(0, \infty)}\left(R, R^{+}\right)=\bigcup_{I} \mathcal{Y}_{I}\left(R, R^{+}\right)$is an honest adic space.

Proof. The same arguments as for Proposition 3.2.1 apply.

Again, there is a totally discontinuous action $\varphi$ of Frobenius.

Definition 3.3.2. The relative Fargues-Fontaine curve $\mathcal{X}\left(R, R^{+}\right)$is the quotient $\mathcal{Y}_{(0, \infty)}\left(R, R^{+}\right) / \varphi^{\mathbb{Z}}$.

As before, there is a line bundle $\mathcal{O}_{\mathcal{X}\left(R, R^{+}\right)}(d)$ for any $d \in \mathbb{Z}$, and one can form the scheme

$$
X(R)=\operatorname{Proj}\left(\oplus_{d \geq 0} H^{0}\left(\mathcal{X}\left(R, R^{+}\right), \mathcal{O}_{\mathcal{X}\left(R, R^{+}\right)}(d)\right)\right) 12
$$

This comes with a map of locally ringed topological spaces $\mathcal{X}\left(R, R^{+}\right) \rightarrow X(R)$, and one has a relative GAGA result.

\footnotetext{
${ }^{10}$ We will not fix a perfectoid base field inside $R$, although one can always find one.

${ }^{11}$ One can check that $\mathcal{R}_{R}^{[s, r]}$ depends only on $R$, and not on $R^{+}$.

${ }^{12}$ As notation suggests, this does not depend on $R^{+}$.
} 
Theorem 3.3.3 ([KL15, Theorem 8.7.7]). The pullback functor from vector bundles on $X(R)$ to vector bundles on $\mathcal{X}\left(R, R^{+}\right)$is an equivalence of categories.

Moreover, we can define $\tilde{\mathcal{R}}_{R}^{r}$ as the inverse limit of the Banach algebras $\tilde{\mathcal{R}}_{R}^{[s, r]}$ as $s$ runs over $(0, r]$ and the relative Robba ring $\tilde{\mathcal{R}}_{R}$ as the direct limit of the Fréchet algebras $\tilde{\mathcal{R}}_{R}^{r}$ over $r>0$. Again, a $\varphi$-module over $\tilde{\mathcal{R}}_{R}$ is a finite projective $\tilde{\mathcal{R}}_{R}$-module $M$ equipped with a $\varphi$-linear automorphism.

Theorem 3.3.4 (KL15, Theorems 6.3.12, 8.7.7]). There is an equivalence of categories

$$
\left\{\text { Vector bundles on } \mathcal{X}\left(R, R^{+}\right)\right\} \simeq\left\{\varphi \text {-modules over } \tilde{\mathcal{R}}_{R}\right\} \text {. }
$$

3.4. The mixed characteristic affine Grassmannian. Our goal in this section is to construct an isomorphism between the flag variety $\mathscr{F} \ell_{G, \mu}$ and the Schubert cell corresponding to $\mu$ in the $B_{\mathrm{dR}}^{+}$-Grassmannian for $G$, assuming that $\mu$ is minuscule. This is an analogue of a classical statement about the usual affine Grassmannian.

Throughout this section, $G$ is a connected reductive group over $\mathbb{Q}_{p}$. First, we define the version of the affine Grassmannian that we will consider. Let $\left(R, R^{+}\right)$ a perfectoid affinoid algebra over $\mathbb{Q}_{p}$, in the sense of [KL15, Definition 3.6.1] 13 One has the surjective map $\theta: W\left(R^{b+}\right) \rightarrow R^{+}$, whose kernel is generated by a non-zerodivisor $\xi \in W\left(R^{+}\right)$. Then $\mathbb{B}_{\mathrm{dR}, R}^{+}$is defined as the $\xi$-adic completion of $W\left(R^{b+}\right)[1 / p]$, and $\mathbb{B}_{\mathrm{dR}, R}=\mathbb{B}_{\mathrm{dR}, R}^{+}\left[\xi^{-1}\right]$. We note that, as notation suggests, these rings are independent of the choice of $R^{+}$.

Definition 3.4.1. Let $\mathrm{Gr}_{G}^{B_{\mathrm{dR}}^{+}}$be the functor associating to any perfectoid affinoid $\mathbb{Q}_{p}$-algebra $\left(R, R^{+}\right)$the set of $G$-torsors over $\mathrm{Spec} \mathbb{B}_{\mathrm{dR}, R}^{+}$trivialized over Spec $\mathbb{B}_{\mathrm{dR}, R}$, up to isomorphism.

We refer to Wei14 for a more thorough discussion of this object, in the case $G=G L_{n}$.

If $\left(R, R^{+}\right)=\left(K, K^{+}\right)$where $K$ is a perfectoid field, then $\mathbb{B}_{\mathrm{dR}, K}^{+}$is a complete discrete valuation ring, abstractly isomorphic to $K[[\xi]]$. In that case, one sees that

$$
\operatorname{Gr}_{G}^{B_{\mathrm{dR}}^{+}}\left(K, K^{+}\right)=G\left(\mathbb{B}_{\mathrm{dR}, K}\right) / G\left(\mathbb{B}_{\mathrm{dR}, K}^{+}\right) .
$$

In particular, assume that $K=C$ is algebraically closed, and fix an embedding $\overline{\mathbb{Q}}_{p} \rightarrow C$. Then, using the Cartan decomposition

$$
G\left(\mathbb{B}_{\mathrm{dR}, C}\right)=\bigsqcup_{\mu \in X_{*}(G)_{\mathrm{dom}}} G\left(\mathbb{B}_{\mathrm{dR}, C}^{+}\right) \mu(\xi)^{-1} G\left(\mathbb{B}_{\mathrm{dR}, C}^{+}\right)
$$

(where the induced embedding $\overline{\mathbb{Q}}_{p} \hookrightarrow \mathbb{B}_{\mathrm{dR}, C}^{+}$is used to define $\mu(\xi)$ for a cocharacter $\left.\mu: \mathbb{G}_{m} \rightarrow G_{\overline{\mathbb{Q}}_{p}}\right)$, we can associate an element of $\mu(x) \in X_{*}(G)_{\text {dom }}$ to any point of $x \in \operatorname{Gr}_{G}^{B_{\mathrm{dR}}^{+}}\left(C, \mathcal{O}_{C}\right)$. This is the decomposition into Schubert cells 14

Now, we fix a conjugacy class $\mu$ of cocharacters $\mathbb{G}_{m} \rightarrow G_{\overline{\mathbb{Q}}_{p}}$, defined over $E$. In the following, we assume that $R$ is an $E$-algebra. Any choice of representative $\mu: \mathbb{G}_{m} \rightarrow G_{\overline{\mathbb{Q}}_{p}}$ in this conjugacy class determines an ascending filtration Fil. $(\mu)$ on $\operatorname{Rep}_{\overline{\mathbb{Q}}_{p}} G$, where $\operatorname{Fil}_{m}(\mu)$ is the direct sum of all subspaces where $\mu$ acts through

\footnotetext{
${ }^{13}$ If $R$ contains a perfectoid field, this agrees with the definition of [Sch12a, and this case would suffice for our discussion here.

${ }^{14}$ We have inserted a slightly nonstandard sign in $\mu(\xi)^{-1}$.
} 
weights $m^{\prime} \geq-m 15$ Let $\mathscr{F} \ell_{G, \mu} / E$ be the rigid-analytic flag variety parametrizing all such filtrations. The choice of $\mu$ identifies $\mathscr{F} \ell_{G, \mu}=G / P_{\mu}$, where $P_{\mu} \subset G$ is the stabilizer of Fil• $(\mu)$.

Definition 3.4.2. Let $\mathrm{Gr}_{G, \mu}^{B_{\mathrm{dR}}^{+}} \subset \mathrm{Gr}_{G}^{B_{\mathrm{dR}}^{+}} \otimes_{\mathbb{Q}_{p}} E$ be the subfunctor sending a perfectoid affinoid E-algebra $\left(R, R^{+}\right)$to the set of those $G$-torsors over Spec $\mathbb{B}_{\mathrm{dR}, R}^{+}$trivialized over $\operatorname{Spec} \mathbb{B}_{\mathrm{dR}, R}$ whose relative position $\mu(x)$ is given by $\mu$, for all $x \in \operatorname{Spa}\left(R, R^{+}\right)$.

Proposition 3.4.3. There is a natural Bialynicki-Birula map

$$
\pi_{G, \mu}: \operatorname{Gr}_{G, \mu}^{B+} \rightarrow \mathscr{F} \ell_{G, \mu}
$$

where we regard $\mathscr{F} \ell_{G, \mu}$ as a functor on perfectoid affinoid E-algebras.

Proof. By the Tannakian formalism, it is enough to prove this result in the case $G=G L_{n}$. In that case, write $\mu=\left(k_{1}, \ldots, k_{n}\right)$ as a tuple of $n$ integers, $k_{1} \geq k_{2} \geq$ $\ldots \geq k_{n}$. The functor $\operatorname{Gr}_{G L_{n}}^{B_{\mathrm{dR}}^{+}}$parametrizes $\mathbb{B}_{\mathrm{dR}, R^{-}}^{+}$-lattices $\Lambda \subset \mathbb{B}_{\mathrm{dR}, R}^{n}$, i.e. finite projective submodules such that $\Lambda[1 / \xi]=\mathbb{B}_{\mathrm{dR}, R}^{n}$. Any such lattice gives rise to a filtration on $R^{n}$ by setting

$$
\mathrm{Fil}_{m} R^{n}=\left(\left(\mathbb{B}_{\mathrm{dR}, R}^{+}\right)^{n} \cap \xi^{-m} \Lambda\right) /\left(\left(\xi \mathbb{B}_{\mathrm{dR}, R}^{+}\right)^{n} \cap \xi^{-m} \Lambda\right) .
$$

Using the fact that a finitely generated $R$-module $M$ for which $\operatorname{dim}_{C(x)} M \otimes_{R} C(x)$ is the same for all $x=\mathrm{Spa}\left(C(x), \mathcal{O}_{C(x)}\right) \rightarrow \operatorname{Spa}\left(R, R^{+}\right)$is finite projective, cf. KL15. Proposition 2.8.4], one verifies that $R^{n} / \mathrm{Fil}_{m} R^{n}$ is a finite projective $R$-module for any $m$.

Note that Fil. $R^{n}$ is an increasing filtration, where the rank of $\mathrm{Fil}_{m} R^{n}$ is given by the largest $i$ such that $k_{i} \geq-m$. The same type of filtrations is parametrized by $\mathscr{F} \ell_{G, \mu}$, as desired.

Lemma 3.4.4. Assume that $\mu$ is minuscule, and that $\left(R, R^{+}\right)=\left(K, K^{+}\right)$, where $K / E$ is a perfectoid field. Then

$$
\pi_{G, \mu}: \operatorname{Gr}_{G, \mu}^{B_{\mathrm{dR}}^{+}}\left(K, K^{+}\right) \rightarrow \mathscr{F} \ell_{G, \mu}\left(K, K^{+}\right)
$$

is a bijection.

Proof. Recall that $\mathbb{B}_{\mathrm{dR}, K}^{+}$is a complete discrete valuation ring with residue field $K$. By the Cohen structure theorem, we may choose an isomorphism $\mathbb{B}_{\mathrm{dR}, K}^{+} \cong K[[\xi]]$. This identifies

$$
\operatorname{Gr}_{G, \mu}^{B_{\mathrm{dR}}^{+}}\left(K, K^{+}\right)=G(K((\xi))) / G(K[[\xi]]),
$$

and the Bialynicki-Birula morphism becomes the Bialynicki-Birula morphism for the usual affine Grassmannian for $G / \mathbb{Q}_{p}$. This is known to be an isomorphism, cf. e.g. [NP01, Lemme 6.2].

Theorem 3.4.5. Assume that $\mu$ is minuscule. Then the Bialynicki-Birula morphism

is an isomorphism.

$$
\pi_{G, \mu}: \operatorname{Gr}_{G, \mu}^{B_{\mathrm{dR}}^{+}} \rightarrow \mathscr{F} \ell_{G, \mu}
$$

\footnotetext{
${ }^{15}$ One reason the minus sign appears here is for consistenty with the global definitions, where type $(p, q)$ refers to characters $z \mapsto z^{-p} \bar{z}^{-q}$.
} 
Proof. In the proof, we will use the Tannakian formalism. This interprets $\mathrm{Gr}_{G}^{B_{\mathrm{dR}}^{+}}$as the associations mapping any $V \in \operatorname{Rep} G$ to a lattice $\Lambda_{V} \subset V \otimes \mathbb{B}_{\mathrm{dR}}$, compatibly with tensor products and short exact sequences.

First, let us check injectivity of $\pi_{G, \mu}$. Thus, take two $\left(R, R^{+}\right)$-valued points $x, y \in \operatorname{Gr}_{G, \mu}^{B_{\mathrm{dR}}^{+}}\left(R, R^{+}\right)$which are sent to the same point of $\mathscr{F} \ell_{G, \mu}$. We have to show that the corresponding lattices $\Lambda_{V, x}, \Lambda_{V, y}$ agree for all $V \in \operatorname{Rep} G$. But at any point $z \in \operatorname{Spa}\left(R, R^{+}\right)$with completed residue field $K(z)$, Lemma 3.4.4 implies that

$$
\Lambda_{V, x} \otimes_{\mathbb{B}_{\mathrm{dR}, R}^{+}} \mathbb{B}_{\mathrm{dR}, K(z)}^{+}=\Lambda_{V, y} \otimes_{\mathbb{B}_{\mathrm{dR}, R}^{+}} \mathbb{B}_{\mathrm{dR}, K(z)}^{+} .
$$

One concludes that $\Lambda_{V, x}=\Lambda_{V, y}$ by applying the following lemma to all elements of $\Lambda_{V, x}$, and $\Lambda_{V, y}$.

Lemma 3.4.6. Let $\Lambda$ be a finite projective $\mathbb{B}_{\mathrm{dR}, R^{-}}^{+}$odule, and $a \in \Lambda \otimes_{\mathbb{B}_{\mathrm{dR}, R}^{+}} \mathbb{B}_{\mathrm{dR}, R}$ any element. Assume that for all $z \in \operatorname{Spa}\left(R, R^{+}\right)$with completed residue field $K(z)$, $a \in \Lambda \otimes_{\mathbb{B}_{\mathrm{dR}, R}^{+}} \mathbb{B}_{\mathrm{dR}, K(z)}^{+}$. Then $a \in \Lambda$.

Proof. We may choose $m \geq 0$ minimal such that $a \in \xi^{-m} \Lambda$, and assume $m>0$ for contradiction. Then $a$ induces a nonzero element $\bar{a}$ of the finite projective $R$ module $\xi^{-m} \Lambda / \xi^{-m+1} \Lambda$. By assumption, the specialization of $\bar{a}$ to $K(z)$ vanishes for all $z \in \operatorname{Spa}\left(R, R^{+}\right)$. But an element of $R$ vanishing at all points of $\operatorname{Spa}\left(R, R^{+}\right)$ is trivial, as $R$ is reduced.

Now, to prove surjectivity, we first observe that $\mathrm{Gr}_{G}^{B_{\mathrm{dR}}^{+}}$is in fact a sheaf for the pro-étale topology used in Sch13c 16 More precisely, we allow covers $Y=$ $\operatorname{Spa}\left(S, S^{+}\right) \rightarrow X=\operatorname{Spa}\left(R, R^{+}\right)$which can be written as a composite $Y \rightarrow Y_{0} \rightarrow X$, where $Y \rightarrow Y_{0}$ is an inverse limit of finite étale surjective maps, and $Y_{0} \rightarrow X$ is étale. This pro-étale topology of perfectoid spaces is defined in [KL15, §9.2]. The descent result we need is KL15, Theorem 9.2.15]. Indeed, using the Tannakian formalism, it is enough to prove that one can glue finite projective $\mathbb{B}_{\mathrm{dR}, R^{-}}^{+}$odules in the pro-étale topology. As $\mathbb{B}_{\mathrm{dR}, R}^{+}$is $\xi$-adically complete with $\xi$ a non-zerodivisor and $\mathbb{B}_{\mathrm{dR}, R}^{+} / \xi=R$, a standard argument reduces us to gluing finite projective $R$ modules, which is precisely [KL15, Theorem 9.2.15].

Thus, we see that it is enough to construct, for any representation $V$ of $G$, a $\mathbb{B}_{\mathrm{dR}^{-}}^{+}$ local system $\mathbb{M}_{V} \subset V \otimes \mathbb{B}_{\mathrm{dR}}$ on the pro-étale site of $\mathscr{F} \ell_{G, \mu}$, compatibly with tensor products and short exact sequences, which maps to the correct filtration under the Bialynicki-Birula morphism. Indeed, by pullback, this will induce a similar $\mathbb{B}_{\mathrm{dR}}^{+}$ local system on the pro-étale site of $\operatorname{Spa}\left(R, R^{+}\right)$for any $\left(R, R^{+}\right)$-valued point of $\mathscr{F} \ell_{G, \mu}$, which by the descent result above gives an $\left(R, R^{+}\right)$-point of $\mathrm{Gr}_{G, \mu}^{B_{\mathrm{dR}}^{+}}$.

Now note that any representation $V$ of $G$ gives rise to a filtered module with integrable connection $\left(V \otimes \mathcal{O}_{\mathscr{F} \ell_{G, \mu}}, \mathrm{id} \otimes \nabla\right.$, Fil_••), where Fil• is the universal ascending filtration parametrized by $\mathscr{F} \ell_{G, \mu}$ (so that Fil_• is a descending filtration). Because $\mu$ is minuscule, this filtered module with integrable connection satisfies Griffiths transversality (with the same proof as in the complex case, cf. Del79, Proposition 1.1.14]). Now [Sch13c, Proposition 7.9] constructs a corresponding $\mathbb{B}_{\mathrm{dR}}^{+}$-local system $\mathbb{M}_{V} \subset V \otimes \mathbb{B}_{\mathrm{dR}}$ on the pro-étale site of $\mathscr{F} \ell_{G, \mu}$, and this construction is compatible with tensor products and short exact sequences. One verifies that the induced filtration is correct, finishing the proof.

\footnotetext{
${ }^{16}$ It is also a sheaf for stronger topologies as used in Wei14, but we do not need this here.
} 
3.5. Vector bundles over $\mathcal{X}$ and the Newton stratification. The goal of this subsection is to define the Newton stratification on $\mathscr{F} \ell_{G, \mu}$, where $G / \mathbb{Q}_{p}$ is a reductive group, and $\mu$ is a conjugacy class of minuscule cocharacters, defined over the reflex field $E$. The idea is that, given a $\left(C, \mathcal{O}_{C}\right)$-point of $\mathscr{F} \ell_{G, \mu} \cong \mathrm{Gr}_{G, \mu}^{B_{\mathrm{dR}}^{+}}$, one can modify the trivial $G$-bundle over $\mathcal{X}_{C^{b}}$ along $\infty$ to obtain a new $G$-bundle over $\mathcal{X}_{C^{b}}$, and therefore (by Fargues' theorem) an element of $B(G)$.

Fix any perfectoid affinoid $\left(R, R^{+}\right)$over $\mathbb{Q}_{p}$. We recall how to construct a vector

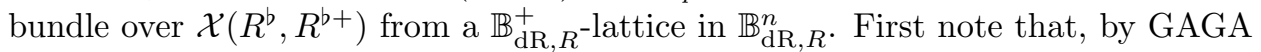
for the curve, it is enough to define a vector bundle on a scheme version $X\left(R^{b}\right)$ of $\mathcal{X}\left(R^{b}, R^{b+}\right)$. Let $Z$ be the image of the canonical closed immersion

$$
i_{\infty}: \text { Spec } R \rightarrow X\left(R^{b}\right) \text {. }
$$

Then Spec $\mathbb{B}_{\mathrm{dR}, R}^{+}$is the completion of $X\left(R^{b}\right)$ along $Z$. Moreover, Spec $\mathbb{B}_{\mathrm{dR}, R}$ can be identified with the fiber product of Spec $\mathbb{B}_{\mathrm{dR}, R}^{+}$and the complement of $Z$ over $X\left(R^{b}\right)$.

Theorem 3.5.1 ([KL15, Theorem 8.9.6]). There is an equivalence between the category of vector bundles over $X\left(R^{b}\right)$ (or over $\mathcal{X}\left(R^{b}, R^{b+}\right)$ ) and the category of triples $\left(M_{1}, M_{2}, \iota\right)$, where $M_{1}$ is a vector bundle on $X\left(R^{b}\right) \backslash Z, M_{2}$ is a vector bundle over Spec $\mathbb{B}_{\mathrm{dR}, R}^{+}$, and $\iota$ is an isomorphism between $\left.M_{1}\right|_{\text {Spec } \mathbb{B}_{\mathrm{dR}, R}}$ and $\left.M_{2}\right|_{\mathrm{Spec} \mathbb{B}_{\mathrm{dR}, R}}$. This equivalence is compatible with tensor products and short exact sequences.

In particular, one gets a functor from $\mathbb{B}_{\mathrm{dR}, R}^{+}$-lattices in $\mathbb{B}_{\mathrm{dR}, R}^{n}$ by gluing it to the trivial rank $n$ vector bundle on $X\left(R^{b}\right) \backslash Z$.

Corollary 3.5.2. For any perfectoid affinoid $\mathbb{Q}_{p}$-algebra $\left(R, R^{+}\right)$, there is a natural map

$$
\mathcal{E}: \operatorname{Gr}_{G}^{B_{\mathrm{dR}}^{+}}\left(R, R^{+}\right) \rightarrow\left\{G \text {-bundles over } \mathcal{X}\left(R^{\mathrm{b}}, R^{\mathrm{b}+}\right)\right\} .
$$

Proof. If $G=G L_{n}$, this follows from the discussion above. In general, it follows from the Tannakian formalism.

In particular, consider the case where $\left(R, R^{+}\right)=\left(C, \mathcal{O}_{C}\right)$, with $C / \mathbb{Q}_{p}$ complete and algebraically closed, and $\mathcal{O}_{C} \subset C$ its ring of integers; moreover, fix an embed$\operatorname{ding} \overline{\mathbb{Q}}_{p} \hookrightarrow C$. Using Fargues' classification of $G$-bundles, Theorem 3.2.9 one gets a composite map

$$
b(\cdot): \operatorname{Gr}_{G}^{B_{\mathrm{dR}}^{+}}\left(C, \mathcal{O}_{C}\right) \rightarrow B(G): x \mapsto b(\mathcal{E}(x))
$$

classifying the isomorphism class of the associated $G$-bundle $\mathcal{E}(x)$. We will need to know the following compatibility between $\mu$ and $b$.

Proposition 3.5.3. Let $G$ be any reductive group over $\mathbb{Q}_{p}$, and $\mu$ any conjugacy class of cocharacters (not necessarily minuscule). For any $x \in \operatorname{Gr}_{G, \mu}^{B_{\mathrm{dR}}^{+}}\left(C, \mathcal{O}_{C}\right)$ with $b=b(\mathcal{E}(x))$, one has $b \in B\left(G, \mu^{-1}\right)$.

Proof. Unraveling the definition of $B\left(G, \mu^{-1}\right)$, we have to prove two separate statements. The first statement is $\nu_{b} \preceq \overline{\mu^{-1}}$ as elements of $\left(X_{*}(G) \otimes \mathbb{Q}\right)_{\mathrm{dom}}^{\Gamma}$. This reduces to the case of $G=G L_{n}$ by [RR96, Lemma 2.2]. In that case, the statement is the following. 
Lemma 3.5.4. Let $\mathcal{E}$ be a vector bundle of rank $n$ over $X_{C^{b}}$, together with a trivialization outside the point $\infty$. Its relative position from the trivial bundle on $X_{C^{b}}$ is measured by a cocharacter $\mu(\mathcal{E})$ of $G L_{n}$. Let $\nu_{\mathcal{E}} \in\left(X_{*}\left(G L_{n}\right) \otimes \mathbb{Q}\right)_{\mathrm{dom}}$ be the Newton polygon of $\mathcal{E}$, with slopes $\left\{\lambda_{i} \mid \mathcal{E} \cong \bigoplus_{i} \mathcal{O}_{X_{C^{b}}}\left(\lambda_{i}\right)\right\}$. One has the inequality

$$
\nu_{\mathcal{E}} \preceq \mu(\mathcal{E}),
$$

i.e. "The Newton polygon of $\mathcal{E}$ lies above its Hodge polygon" 17

Proof. We adapt the original argument in Kat79. By considering exterior powers of vector bundles, it suffices to check that

(1) the Newton and Hodge slopes match for the top exterior power of $\mathcal{E}$, and

(2) the first slope of the Newton polygon of $\mathcal{E}$ always lies above the first slope of the Hodge polygon of $\mathcal{E}$.

The fact that the Hodge and Newton slopes match in the case of line bundles on $\mathcal{X}_{F F, C}$ is a direct verification: The modification $\mathcal{E}$ is given by the lattice $\mathcal{E} \otimes_{\mathcal{O}_{X^{b}}}$ $\mathbb{B}_{\mathrm{dR}, C}^{+}=\xi^{-d} \mathbb{B}_{\mathrm{dR}, C}$ for a unique $d \in \mathbb{Z}$, and in fact $\mu(\mathcal{E})=d \in X_{*}\left(G L_{1}\right)=\mathbb{Z}$ in our normalization. The resulting line bundle is given by $\mathcal{O}_{X^{b}}(d)$, which is of slope $d$, as desired.

For the second part, up to twisting, we may assume that the first slope of the Hodge polygon is 0, in particular all Hodge slopes are nonnegative. This implies that

$$
\left(\mathbb{B}_{\mathrm{dR}, C}^{+}\right)^{n} \subseteq \mathcal{E} \otimes_{\mathcal{O}_{X_{C}}} \mathbb{B}_{\mathrm{dR}, C}^{+} .
$$

This, in turn, implies that the trivialization of $\mathcal{E}$ away from $\infty$ extends to an injection $\mathcal{O}_{X_{C^{b}}}^{n} \hookrightarrow \mathcal{E}$. We have to show that all slopes of $\mathcal{E}$ are nonnegative, so assume for contradiction that there is a quotient $\mathcal{E} \rightarrow \mathcal{O}_{X_{C^{b}}}(\lambda)$ with $\lambda<0$. This induces a nonzero map $\mathcal{O}_{X_{C^{b}}}^{n} \rightarrow \mathcal{O}_{X_{C^{b}}}(\lambda)$. On the other hand, there are no nonzero maps $\mathcal{O}_{X_{C^{b}}} \rightarrow \mathcal{O}_{X_{C^{b}}}(\lambda)$ by [FF14].

The other part of the condition $b \in B\left(G, \mu^{-1}\right)$ concerns the Kottwitz map, and is given by the following lemma.

Lemma 3.5.5. The composition $\mathrm{Gr}_{G, \mu}^{B_{\mathrm{dR}}^{+}}\left(C, \mathcal{O}_{C}\right) \rightarrow B(G) \stackrel{\kappa}{\longrightarrow} \pi_{1}(G)_{\Gamma}$ is constant, and equal to $-\mu^{b}$.

Proof. We note that the map in question is functorial in $(G, \mu)$. We first reduce to the case where $G$ has simply connected derived group by making a central extension $\tilde{G} \rightarrow G$ (cf. [Kot85, 5.6]); picking any lift $\tilde{\mu}$ of $\mu$, the resulting map

$$
\mathrm{Gr}_{\tilde{G}, \tilde{\mu}}^{B_{\mathrm{dR}}^{+}}\left(C, \mathcal{O}_{C}\right) \rightarrow \mathrm{Gr}_{G, \mu}^{B_{\mathrm{dR}}^{+}}\left(C, \mathcal{O}_{C}\right)
$$

is surjective, as follows from the Cartan decomposition, so it is enough to prove the result for $(\tilde{G}, \tilde{\mu})$.

Now if $G$ has simply connected derived group $G^{\text {der }}$, then $T=G / G^{\text {der }}$ is a torus for which $\pi_{1}(G)_{\Gamma} \rightarrow \pi_{1}(T)_{\Gamma}$ is an isomorphism; thus, we are reduced to the case of a torus.

\footnotetext{
${ }^{17}$ We remind the reader that the correspondence between isocrystals and vector bundles on $X_{C^{b}}$ reverses slopes, so that this statement translates into $b(\mathcal{E})^{-1} \in B\left(G L_{n}, \mu(\mathcal{E})\right)$, which is equivalent to $b(\mathcal{E}) \in B\left(G L_{n}, \mu(\mathcal{E})^{-1}\right)$.
} 
If $G=T$ is a torus, we may find a surjection $\tilde{T} \rightarrow T$, where $\tilde{T}$ is a product of induced tori $\operatorname{Res}_{K / \mathbb{Q}_{p}} \mathbb{G}_{m}$. Arguing as before, we are reduced to the case of $\tilde{T}$, and then to the case $\tilde{T}=\operatorname{Res}_{K / \mathbb{Q}_{p}} \mathbb{G}_{m}$. In that case, $\pi_{1}(\tilde{T})_{\Gamma}=\mathbb{Z}$ (cf. $\operatorname{Kot} 85$, Lemma $2.2])$, which is torsion-free, so it is enough to identify the image in $\pi_{1}\left(\mathbb{G}_{m}\right)=\mathbb{Z}$ under the norm map $\operatorname{Norm}_{K / \mathbb{Q}_{p}}: \tilde{T} \rightarrow \mathbb{G}_{m}$. Finally, we are reduced to the case $G=\mathbb{G}_{m}$, which is part of Lemma 3.5.4

Now fix a minuscule $\mu$ as above, defined over $E$. The inverse of the isomorphism $\pi_{G, \mu}$ in Theorem 3.4.5 gives rise to a composition

$$
\mathcal{E}: \mathscr{F} \ell_{G, \mu}\left(R, R^{+}\right) \rightarrow \operatorname{Gr}_{G, \mu}^{B_{\mathrm{dR}}^{+}}\left(R, R^{+}\right) \rightarrow\left\{G \text {-bundles over } \mathcal{X}\left(R^{b}, R^{b+}\right)\right\} .
$$

Definition 3.5.6. The map

$$
\left|\mathscr{F} \ell_{G, \mu}\right| \rightarrow B(G)
$$

sends any $\left(C, C^{+}\right)$-valued point $x \in \mathscr{F} \ell_{G, \mu}\left(C, C^{+}\right)$, where $C$ is a complete algebraically closed extension of $E$ and $C^{+} \subset C$ is an open and bounded valuation subring, to the isomorphism class of the associated $G$-bundle $\mathcal{E}(x)$, which by Theorem 3.2 .9 is given by an element of $B(G)$. $b$.

For any $b \in B(G)$, we let $\mathscr{F} \ell_{G, \mu}^{b} \subset \mathscr{F} \ell_{G, \mu}$ be the subset of all points with image

One easily checks that this map is well-defined as a map on $\left|\mathscr{F} \ell_{G, \mu}\right|$, i.e. is independent of the choice of complete algebraically closed extension of the residue field at any point. We remark that by definition a higher rank point has the same image as its maximal, rank 1, generalization, and therefore the map factors over the maximal hausdorff quotient of $\left|\mathscr{F} \ell_{G, \mu}\right|$, which can be identified with the topological space $\mathscr{F} \ell_{G, \mu}^{\text {Berk }}$ underlying the corresponding Berkovich space.

Proposition 3.5.7. (1) The map $b(\cdot):\left|\mathscr{F}_{G, \mu}\right| \rightarrow B(G)$ is lower semicontinuous.

(2) The image of the map $b(\cdot):\left|\mathscr{F} \ell_{G, \mu}\right| \rightarrow B(G)$ is contained in the set of $\mu^{-1}$-admissible elements $B\left(G, \mu^{-1}\right)$.

Remark 3.5.8. In Rap15, Proposition A.9], based on the discussion here, it is proved that in fact the image of $\left|\mathscr{F} \ell_{G, \mu}\right| \rightarrow B\left(G, \mu^{-1}\right)$ is all of $B\left(G, \mu^{-1}\right)$.

Proof. The second part follows from Proposition 3.5 .3 above. For the first part, by the definition of the partial ordering on $B(G)$, and the fact that the Kottwitz map is constant by the second part, it remains to prove semicontinuity of the Newton map. We may pick an affinoid perfectoid space $\operatorname{Spa}\left(R, R^{+}\right)$with a map to $\mathscr{F} \ell_{G, \mu}$ which is a topological quotient map, by using a pro-étale cover. It is then enough to show that the composite map $\left|\operatorname{Spa}\left(R, R^{+}\right)\right| \rightarrow\left|\mathscr{F} \ell_{G, \mu}\right| \rightarrow B(G)$ is lower semicontinuous. But semicontinuity of the Newton map can be checked on representations of $G$ (cf. RR96, Lemma 2.2]), so pick a representation of $G$. We get a corresponding vector bundle over $\mathcal{X}\left(R^{b}, R^{b+}\right)$. Now, the result follows from Theorem 7.4.5 of KL15, using Corollary 3.3.4. 
Corollary 3.5.9. The strata $\mathscr{F} \ell_{G, \mu}^{b}$ are locally closed in $\mathscr{F} \ell_{G, \mu}$. More precisely, the stratum corresponding to the basic element is open in $\mathscr{F} \ell_{G, \mu}$, and the strata

$$
\mathscr{F} \ell_{\bar{G}, \mu}^{\succeq b}:=\bigsqcup_{b \preceq b^{\prime}} \mathscr{F} \ell_{G, \mu}^{b^{\prime}}
$$

are closed.

Proof. This follows immediately from Proposition 3.5.7. 


\section{The geometry of NeWton strata And Igusa varieties}

In this section, we will return to the global setup, but will in addition assume that the Shimura datum $(G, X)$ is of PEL type, and has good reduction at $p$. This means that they will admit smooth integral models which are moduli spaces of abelian varieties equipped with polarizations, endomorphisms and level structure. Our goal is to understand the fibers of the Hodge-Tate period map

$$
\pi_{H T}: \mathcal{S}_{K^{p}} \rightarrow \mathscr{F} \ell_{G, \mu}
$$

defined in Theorem 2.1.3 in terms of the Igusa varieties introduced by Mantovan, Man05.

We start with some preliminaries on $p$-divisible groups, which recall material from SW13 as well as a construction of Chai and Oort. We then express the Newton strata in $\mathcal{S}_{K^{p}}$ in terms of Rapoport-Zink spaces and Igusa varieties, in the spirit of Man05].

4.1. Preliminaries on $p$-divisible groups. We recall the notions of Tate module and universal cover of a $p$-divisible group as used in [SW13, together with some of their properties. Let Nilp be the category of $\mathbb{Z}_{p}$-algebras on which $p$ is nilpotent. If $R$ is a $p$-adically complete $\mathbb{Z}_{p}$-algebra, let Nilp op be the opposite category to the category of $R$-algebras on which $p$ is nilpotent. A $p$-divisible group $\mathcal{G}$ can be thought of as an fpqc sheaf on $\operatorname{Nilp}_{R}^{\text {op }}$ sending an $R$-algebra $S$ to $\lim _{\longrightarrow} \mathcal{G}\left[p^{n}\right](S)$.

Definition 4.1.1. (1) The fpqc sheaf $T_{p}(\mathcal{G})(S)=\lim _{n} \mathcal{G}\left[p^{n}\right](S)$ on $\operatorname{Nilp}_{R}^{\text {op }}$ is called the (integral) Tate module of $\mathcal{G}$.

(2) The fpqc sheaf $\tilde{\mathcal{G}}(S)=\lim _{p: \mathcal{G} \rightarrow \mathcal{G}} \mathcal{G}(S)$ on $\operatorname{Nilp}_{R}^{\text {op }}$ is called the universal cover of $\mathcal{G}$.

We note that $T_{p}(\mathcal{G})$ is a sheaf of $\mathbb{Z}_{p}$-modules, while $\tilde{\mathcal{G}}=T_{p}(\mathcal{G})[1 / p]$ is a sheaf of $\mathbb{Q}_{p}$-vector spaces. We can canonically identify

$$
T_{p} \mathcal{G}=\mathscr{H} \text { om }\left(\mathbb{Q}_{p} / \mathbb{Z}_{p}, \mathcal{G}\right), \tilde{\mathcal{G}}=\mathscr{H} \text { om }\left(\mathbb{Q}_{p} / \mathbb{Z}_{p}, \mathcal{G}\right)[1 / p] .
$$

Proposition 4.1.2. $\quad$ (1) If $\mathcal{G}$ is connected, then it is representable by an affine formal scheme with finitely generated ideal of definition. If Lie $\mathcal{G}$ is free of dimension $r$ then

$$
\mathcal{G} \simeq \operatorname{Spf} R\left[\left[x_{1}, \ldots, x_{r}\right]\right] .
$$

(2) If $\rho: \mathcal{G}_{1} \rightarrow \mathcal{G}_{2}$ is an isogeny, then the induced morphism $\tilde{\rho}: \tilde{\mathcal{G}}_{1} \rightarrow \tilde{\mathcal{G}}_{2}$ is an isomorphism.

(3) If $R$ is perfect of characteristic $p, \mathcal{G}$ is connected and Lie $\mathcal{G}$ is free of dimension $r$ then

$$
\tilde{\mathcal{G}} \simeq \operatorname{Spf} R\left[\left[x_{1}^{1 / p^{\infty}}, \ldots, x_{r}^{1 / p^{\infty}}\right]\right] .
$$

(4) If $R$ is perfect of characteristic $p, \mathcal{G}$ is connected and Lie $\mathcal{G}$ is free of dimension $r$ then

$$
T_{p} \mathcal{G} \simeq \operatorname{Spec} R\left[\left[x_{1}^{1 / p^{\infty}}, \ldots, x_{r}^{1 / p^{\infty}}\right]\right] /\left(x_{1}, \ldots, x_{r}\right) .
$$

Proof. The first part is proved in Mes72. The remaining results are proved in [SW13: the second and third parts in Proposition 3.1.3 and the fourth part 
follows from the first part, the third part and the short exact sequence of sheaves on $\mathrm{Nilp}_{R}^{\text {op }}$ given by

$$
0 \rightarrow T_{p} \mathcal{G} \rightarrow \tilde{\mathcal{G}} \rightarrow \mathcal{G} \rightarrow 0
$$

(This short exact sequence is a restatement of Proposition 3.3.1 of [SW13] in the case when $\mathcal{G}$ is connected: the Tate module is the closed subfunctor of $\tilde{\mathcal{G}}$ given by pullback along the natural map $\tilde{\mathcal{G}} \rightarrow \mathcal{G}$ - projection onto the last coordinate - from the zero section in $\mathcal{G}$.)

The universal vector extension $E \mathcal{G}$ of $\mathcal{G}$ is a crystal on the nilpotent crystalline site of $R$ defined in Mes72. Its Lie algebra Lie $E \mathcal{G}$ can be made into a crystal on the crystalline site of $R$ by BBM82, which we will denote by $\mathbb{M}(\mathcal{G})$.

If $\mathcal{G}$ is a $p$-divisible group over $\overline{\mathbb{F}}_{p}$, the Dieudonné module $D(\mathcal{G})$ is obtained by evaluating the crystal $\mathbb{M}(\mathcal{G})$ on the PD thickening $W\left(\overline{\mathbb{F}}_{p}\right) \rightarrow \overline{\mathbb{F}}_{p}$. Then $D(\mathcal{G})[1 / p]$ is an isocrystal over $L$, as defined in Section 4 . Here, the Frobenius $\varphi_{\mathcal{G}}$ on $D(\mathcal{G})[1 / p]$ satisfies

$$
D(\mathcal{G}) \subset \varphi_{\mathcal{G}}(D(\mathcal{G})) \subset p^{-1} D(\mathcal{G}),
$$

and $p \varphi_{\mathcal{G}}$ is the Frobenius usually considered 18 We will call a $p$-divisible group $\mathcal{G}$ over $\overline{\mathbb{F}}_{p}$ isoclinic if the corresponding isocrystal has only one slope. If this slope is given by $-\lambda$, we say that $\mathcal{G}$ is isoclinic of slope $\lambda$, so that $\mu_{p^{\infty}}$ is isoclinic of slope 1.

Given a $p$-divisible group $\mathcal{G}$ over $\overline{\mathbb{F}}_{p}$, we can use the isocrystal $D(\mathcal{G})[1 / p]$ to construct a vector bundle $\mathcal{E}(\mathcal{G})$ over the Fargues-Fontaine curve $\mathcal{X}_{F}$, for any complete algebraically closed nonarchimedean field $F \supset \overline{\mathbb{F}}_{p}$.

Example 4.1.3. If $\mathcal{G}=\mathbb{Q}_{p} / \mathbb{Z}_{p}$, then $D(\mathcal{G})=L$ with $\varphi_{\mathcal{G}}=\sigma$, and $\mathcal{E}(\mathcal{G})=\mathcal{O}_{\mathcal{X}_{F}}$. If $\mathcal{G}=\mu_{p^{\infty}}$, then $D(\mathcal{G})=L$ with $\varphi_{\mathcal{G}}=p^{-1} \sigma$, and $\mathcal{E}(\mathcal{G})=\mathcal{O}_{\mathcal{X}_{F}}(1)$.

On the other hand, one can use the schematic version of the Fargues-Fontaine curve to build a vector bundle corresponding to a $p$-divisible group over $\mathcal{O}_{C} / p$, where $C$ is any complete algebraically closed extension of $\mathbb{Q}_{p}$ with ring of integers $\mathcal{O}_{C} / p$. Define $A_{\text {cris }}$ to be the $p$-adic completion of the PD envelope of the surjection $W\left(\mathcal{O}_{C}^{b}\right) \rightarrow \mathcal{O}_{C} / p$ and $B_{\text {cris }}^{+}:=A_{\text {cris }}[1 / p]$. If $\mathcal{G}$ is a $p$-divisible group over the semiperfect ring $\mathcal{O}_{C} / p$, then its Dieudonné module is a finite projective $A_{\text {cris }}$-module $M(\mathcal{G})$ obtained by evaluating $\mathbb{M}(\mathcal{G})$ on the $\mathrm{PD}$ thickening $A_{\text {cris }} \rightarrow \mathcal{O}_{C} / p$. Then $M(\mathcal{G})[1 / p]$ is a $B_{\text {cris }}^{+}$-module equipped with a Frobenius-semilinear map $\varphi_{\mathcal{G}}$. Recall, cf. [FF14], that the schematic Fargues-Fontaine curve can also be defined as

$$
X_{C^{b}}=\operatorname{Proj}\left(\oplus_{d \geq 0}\left(B_{\text {cris }}^{+}\right)^{\varphi=p^{d}}\right) .
$$

We associate to $\mathcal{G}$ the vector bundle $E(\mathcal{G})$ on $X_{C^{b}}$ corresponding to the graded module

$$
\oplus_{d \geq 0}(M(\mathcal{G})[1 / p])^{\varphi=p^{d}} .
$$

Theorem 4.1.4. (1) For any $p$-divisible group $\mathcal{G}$ over $\mathcal{O}_{C} / p$, there exists a p-divisible group $\mathcal{H}$ over $\overline{\mathbb{F}}_{p}$ and a quasi-isogeny

$$
\rho: \mathcal{H} \times_{\overline{\mathbb{F}}_{p}} \mathcal{O}_{C} / p \rightarrow \mathcal{G}
$$

\footnotetext{
${ }^{18}$ If one uses the usual Frobenius on contravariant Dieudonné theory, then our convention corresponds to defining covariant Dieudonné theory as the literal dual of contravariant Dieudonné theory, i.e. without a Tate twist.
} 
(2) The functor $\mathcal{G} \mapsto E(\mathcal{G})$ from p-divisible groups over $\mathcal{O}_{C} / p$ up to isogeny to vector bundles on $X_{C^{b}}$ is fully faithful, with essential image the vector bundles whose slopes are all between 0 and 1.

(3) Let $\mathcal{G}$ be a p-divisible group over $\overline{\mathbb{F}}_{p}$. Then GAGA for the curve identifies $\mathcal{E}(\mathcal{G})$ with $E(\mathcal{G})$.

Proof. The first two parts are Theorem 5.1.4 of [SW13. The last part is clear.

We now specialize to $p$-divisible groups over a perfect field $k$. (Since every $p$-divisible group over $\mathcal{O}_{C} / p$ is quasi-isogenous to one defined over $\overline{\mathbb{F}}_{p}$, if we are interested in understanding quasi-self-isogenies, it is enough to restrict to this case.) Let $\mathcal{G}, \mathcal{G}^{\prime}$ be two isoclinic $p$-divisible groups over $k$. Our goal is to define an "internal Hom" p-divisible group $\mathcal{H}_{\mathcal{G}, \mathcal{G}^{\prime}}$ over $k$ satisfying the following two properties:

(1) The Tate module $T_{p}\left(\mathcal{H}_{\mathcal{G}, \mathcal{G}^{\prime}}\right)$ can be identified with the sheaf $\mathscr{H}$ om $\left(\mathcal{G}, \mathcal{G}^{\prime}\right)$.

(2) The Dieudonné module $D\left(\mathcal{H}_{\mathcal{G}, \mathcal{G}^{\prime}}\right)[1 / p]$ is equal to

$$
\operatorname{Hom}\left(D(\mathcal{G})[1 / p], D\left(\mathcal{G}^{\prime}\right)[1 / p]\right) \leq 0,
$$

where the latter denotes the internal homomorphism in Dieudonné modules, and we are taking the slope $\leq 0$-part.

In a talk of C.-L. Chai at the Faltings conference 2014, we learnt that a $p$-divisible group satisfying these properties has been defined by Chai and Oort. We explain their construction below.

We define $\mathcal{H}_{\mathcal{G}, \mathcal{G}^{\prime}}$ as an inductive system of finite group schemes. For each $n \geq 1$ consider the commutative group schemes of finite type over $k$ defined as

$$
\mathcal{H}_{n}:=\mathscr{H} \operatorname{om}\left(\mathcal{G}\left[p^{n}\right], \mathcal{G}^{\prime}\left[p^{n}\right]\right) .
$$

For $m \geq n$, there are natural restriction maps

$$
r_{m, n}: \mathcal{H}_{m} \rightarrow \mathcal{H}_{n}
$$

which restrict a homomorphism $\mathcal{G}\left[p^{m}\right] \rightarrow \mathcal{G}^{\prime}\left[p^{m}\right]$ to $\mathcal{G}\left[p^{n}\right] \subset \mathcal{G}\left[p^{m}\right]$. The kernel ker $r_{m, n} \subset \mathcal{H}_{m}$ is a closed subgroup scheme. As we are working over a field, one can form the qoutient $\mathcal{H}_{n}^{(m)}=\mathcal{H}_{m} /$ ker $r_{m, n}$, which is a subgroup scheme of $\mathcal{H}_{n}$. As $m$ increases, they form a descending chain.

Lemma 4.1.5. The subgroup scheme $\mathcal{H}_{n}^{(m)}$ stabilizes for $m \gg 0$; let $\mathcal{H}_{n}^{\prime}=\mathcal{H}_{n}^{(m)}$ for $m$ sufficiently large. Then $\mathcal{H}_{n}^{\prime}$ is a finite group scheme over $k$.

Proof. We may assume that $k$ is algebraically closed. First, we claim that $\mathcal{H}_{n}^{(m)}$ is a finite group scheme for $m \gg 0$. It is enough to see that $\mathcal{H}_{n}^{(m)}(k)$ is finite. By Dieudonné theory, one sees that $\operatorname{Hom}\left(\mathcal{G}, \mathcal{G}^{\prime}\right)$ is a finite free $\mathbb{Z}_{p}$-module, independent of the algebraically closed field $k$. In particular, the image $\mathcal{H}_{n}(k)_{\infty} \subset \mathcal{H}_{n}(k)$ of

$$
\operatorname{Hom}\left(\mathcal{G}, \mathcal{G}^{\prime}\right) \rightarrow \mathcal{H}_{n}(k)
$$

is finite, and independent of $k$. Now the sequence of $\mathcal{H}_{m} \times \mathcal{H}_{n}\left(\mathcal{H}_{n} \backslash \mathcal{H}_{n}(k)_{\infty}\right)$ forms a cofiltered system of quasicompact schemes with affine transition maps and with empty inverse limit. It follows that one of the schemes is already empty, showing that the image of $\mathcal{H}_{m}(k) \rightarrow \mathcal{H}_{n}(k)$ agrees with the finite set $\mathcal{H}_{n}(k)_{\infty}$.

Now, the $\mathcal{H}_{n}^{(m)}$ form a decreasing sequence of finite group schemes over $k$. As such, they are eventually constant, e.g. by looking at their order. 
We define $\iota_{n}: \mathcal{H}_{n} \rightarrow \mathcal{H}_{n+1}$ to be the map given by pre-composition with the multiplication by $p$ map $\mathcal{G}\left[p^{n+1}\right] \rightarrow \mathcal{G}\left[p^{n}\right]$ followed by composition with the inclusion $\mathcal{G}^{\prime}\left[p^{n}\right] \hookrightarrow \mathcal{G}^{\prime}\left[p^{n+1}\right]$.

Lemma 4.1.6. The maps $\iota_{n}: \mathcal{H}_{n} \rightarrow \mathcal{H}_{n+1}$ send $\mathcal{H}_{n}^{\prime}$ into $\mathcal{H}_{n+1}^{\prime}$. The colimit

$$
\mathcal{H}=\mathcal{H}_{\mathcal{G}, \mathcal{G}^{\prime}}=\underset{\iota_{n}}{\lim } \mathcal{H}_{n}^{\prime}
$$

is a p-divisible group over $k$ with $\mathcal{H}\left[p^{n}\right]=\mathcal{H}_{n}^{\prime}$.

Proof. From the commutation between $\iota_{n}$ and $r_{m, n}$, one infers that $\iota_{n}$ sends $\mathcal{H}_{n}^{\prime}$ into $\mathcal{H}_{n+1}^{\prime}$. First, we check that $\iota_{n}: \mathcal{H}_{n}^{\prime} \rightarrow \mathcal{H}_{n+1}^{\prime}$ is injective with image $\mathcal{H}_{n+1}^{\prime}\left[p^{n}\right]$. Let $S$ be any $k$-scheme. If $f: \mathcal{G}\left[p^{n}\right]_{S} \rightarrow \mathcal{G}^{\prime}\left[p^{n}\right]_{S}$ induces the trivial map

$$
\mathcal{G}\left[p^{n+1}\right]_{S} \stackrel{p}{\longrightarrow} \mathcal{G}\left[p^{n}\right]_{S} \stackrel{f}{\longrightarrow} \mathcal{G}^{\prime}\left[p^{n}\right]_{S} \hookrightarrow \mathcal{G}^{\prime}\left[p^{n+1}\right]_{S}
$$

then $f=0$ as the first map is surjective, and the last injective; this proves injectivity of $\iota_{n}$. Now let $f: \mathcal{G}\left[p^{n+1}\right]_{S} \rightarrow \mathcal{G}^{\prime}\left[p^{n+1}\right]_{S}$ be a map killed by $p^{n}$, which for any $m \geq n$ lifts fppf locally to a map $f_{m}: \mathcal{G}\left[p^{m+1}\right]_{S} \rightarrow \mathcal{G}^{\prime}\left[p^{m+1}\right]_{S}$. It follows that $f$ factors uniquely as

$$
\mathcal{G}\left[p^{n+1}\right]_{S} \stackrel{p}{\longrightarrow} \mathcal{G}\left[p^{n}\right]_{S} \stackrel{g}{\longrightarrow} \mathcal{G}^{\prime}\left[p^{n}\right]_{S} \hookrightarrow \mathcal{G}^{\prime}\left[p^{n+1}\right]_{S}
$$

for some $g: \mathcal{G}\left[p^{n}\right] \rightarrow \mathcal{G}^{\prime}\left[p^{n}\right]$, as $f$ has image in the $p^{n}$-torsion, and kills $p^{n} \mathcal{G}\left[p^{n+1}\right]=$ $\mathcal{G}[p]$. Similarly, any lift $f_{m}: \mathcal{G}\left[p^{m+1}\right]_{S} \rightarrow \mathcal{G}^{\prime}\left[p^{m+1}\right]_{S}$ of $f$ is killed by $p^{m}$, which implies that $f_{m}$ factors uniquely through a map $g_{m}: \mathcal{G}\left[p^{m}\right] \rightarrow \mathcal{G}^{\prime}\left[p^{m}\right]$, which necessarily lifts $g$. This shows that $\mathcal{H}_{n}^{\prime}=\mathcal{H}_{n+1}^{\prime}\left[p^{n}\right]$.

Moreover, we need to see that $p: \mathcal{H}_{n+1}^{\prime} \rightarrow \mathcal{H}_{n+1}^{\prime}$ has image $\mathcal{H}_{n}^{\prime}$; by the above, it follows that the image is contained in $\mathcal{H}_{n}^{\prime}$; the resulting map $\mathcal{H}_{n+1}^{\prime} \rightarrow \mathcal{H}_{n}^{\prime}$ is in fact the map $r_{n+1, n}$. By construction of the $\mathcal{H}_{n}^{\prime}$, the map $r_{n+1, n}$ is indeed surjective, finishing the proof.

Lemma 4.1.7. The Tate module $T_{p} \mathcal{H}_{\mathcal{G}, \mathcal{G}^{\prime}}$ can be identified with the sheaf $\mathscr{H}$ om $\left(\mathcal{G}, \mathcal{G}^{\prime}\right)$.

Proof. The Tate module $T_{p} \mathcal{H}_{\mathcal{G}, \mathcal{G}^{\prime}}$ is the inverse limit of $\mathcal{H}_{\mathcal{G}, \mathcal{G}^{\prime}}\left[p^{n}\right] \simeq \mathcal{H}_{n}^{\prime}$ with respect to the $r_{n+1, n}$ maps. This, by definition is the same as the inverse limit of the projective system of $\mathcal{H}_{n}$ 's with respect to the $r_{n+1, n}$ maps, which is the sheaf $\mathscr{H}$ om $\left(\mathcal{G}, \mathcal{G}^{\prime}\right)$.

Lemma 4.1.8. The Dieudonné module $D\left(\mathcal{H}_{\mathcal{G}, \mathcal{G}^{\prime}}\right)[1 / p]$ is equal to

$$
\operatorname{Hom}\left(D(\mathcal{G})[1 / p], D\left(\mathcal{G}^{\prime}\right)[1 / p]\right) \leq 0,
$$

where $\operatorname{Hom}\left(D(\mathcal{G})[1 / p], D\left(\mathcal{G}^{\prime}\right)[1 / p]\right)$ is the internal homomorphism in Dieudonné modules, and we are taking the slope $\leq 0$-part.

Remark 4.1.9. Note that the statement only depends on $\mathcal{G}$ and $\mathcal{G}^{\prime}$ up to quasiisogeny. Chai and Oort prove Lemma 4.1.8 by directly computing the relative Frobenius on $\mathcal{H}_{\mathcal{G}, \mathcal{G}^{\prime}}$ in terms of the relative Frobenius on conveniently chosen $\mathcal{G}$ and $\mathcal{G}^{\prime}$. We give a different proof below. Also, Chai-Oort give an integral version of Lemma 4.1 .8 .

Proof. Let $\mathcal{H}_{D}$ be a $p$-divisible group over $k$ with rational Dieudonné module

$$
\operatorname{Hom}\left(D(\mathcal{G})[1 / p], D\left(\mathcal{G}^{\prime}\right)[1 / p]\right) \leq 0
$$

First, we construct a natural map

$$
\tilde{\mathcal{H}}_{D} \rightarrow \tilde{\mathcal{H}}_{\mathcal{G}, \mathcal{G}^{\prime}}=\mathscr{H} \text { om }\left(\mathcal{G}, \mathcal{G}^{\prime}\right)[1 / p] .
$$


In order to construct such a map, it is enough to construct a functorial map on $R$-valued points, where $R$ is f-semiperfect in the sense of [SW13, Definition 4.1.2], as $\tilde{\mathcal{H}}_{\mathcal{G}, \mathcal{G}^{\prime}}$, like the universal cover of any $p$-divisible group, is represented by a formal scheme which is locally of the form $\operatorname{Spf} S$, where $S$ is an inverse limit of f-semiperfect rings.

Thus, let $R$ be f-semiperfect, with associated $B_{\text {cris }}^{+}(R)$. Then by [SW13, Theorem A], we have

$$
\begin{aligned}
\tilde{\mathcal{H}}_{\mathcal{G}, \mathcal{G}^{\prime}}(R) & =\operatorname{Hom}_{R}\left(\mathcal{G}, \mathcal{G}^{\prime}\right)[1 / p]=\operatorname{Hom}_{B_{\text {cris }}^{+}(R), \varphi}\left(D(\mathcal{G}) \otimes B_{\text {cris }}^{+}(R), D\left(\mathcal{G}^{\prime}\right) \otimes B_{\text {cris }}^{+}(R)\right) \\
& =\left(\operatorname{Hom}\left(D(\mathcal{G})[1 / p], D\left(\mathcal{G}^{\prime}\right)[1 / p]\right) \otimes B_{\text {cris }}^{+}(R)\right)^{\varphi=1},
\end{aligned}
$$

and

$$
\begin{aligned}
\tilde{\mathcal{H}}_{D}(R) & =\operatorname{Hom}_{R}\left(\mathbb{Q}_{p} / \mathbb{Z}_{p}, \mathcal{H}_{D}\right)[1 / p]=\left(D\left(\mathcal{H}_{D}\right) \otimes B_{\text {cris }}^{+}(R)\right)^{\varphi=1} \\
& =\left(\operatorname{Hom}\left(D(\mathcal{G})[1 / p], D\left(\mathcal{G}^{\prime}\right)[1 / p]\right)^{\leq 0} \otimes B_{\text {cris }}^{+}(R)\right)^{\varphi=1} .
\end{aligned}
$$

Now the obvious inclusion

$$
\operatorname{Hom}\left(D(\mathcal{G})[1 / p], D\left(\mathcal{G}^{\prime}\right)[1 / p]\right)^{\leq 0} \subset \operatorname{Hom}\left(D(\mathcal{G})[1 / p], D\left(\mathcal{G}^{\prime}\right)[1 / p]\right)
$$

induces the desired map $\tilde{\mathcal{H}}_{D} \rightarrow \tilde{\mathcal{H}}_{\mathcal{G}, \mathcal{G}^{\prime}}$.

To check that this is an isomorphism, it suffices by the same argument to check on $R$-valued points, where $R$ is f-semiperfect. Thus, it remains to see that

$$
\left(\operatorname{Hom}\left(D(\mathcal{G})[1 / p], D\left(\mathcal{G}^{\prime}\right)[1 / p]\right) \otimes B_{\text {cris }}^{+}(R)\right)^{\varphi=1}=\left(\operatorname{Hom}\left(D(\mathcal{G})[1 / p], D\left(\mathcal{G}^{\prime}\right)[1 / p]\right)^{\leq 0} \otimes B_{\text {cris }}^{+}(R)\right)^{\varphi=1} .
$$

For this, it suffices to see that for any Dieudonné module $D$ with only positive slopes,

$$
\left(D \otimes B_{\text {cris }}^{+}(R)\right)^{\varphi=1}=0 .
$$

For this, using the Dieudonné-Manin classification, we have to see that there are no elements $x \in A_{\text {cris }}(R)$ with $p^{a} \varphi^{b}(x)=x$, where $a, b>0$ are positive integers. Note that $\varphi$ preserves the $p$-adically complete ring $A_{\text {cris }}(R)$; on the other hand, the equation on $x$ implies $x=p^{m a} \varphi^{m b}(x)$ for any $m \geq 1$, so that $x$ is infinitely divisible by $p$, which implies $x=0$.

Corollary 4.1.10. Assume that $\mathcal{G}$ and $\mathcal{G}^{\prime}$ are isoclinic.

(1) If the slope of $\mathcal{G}$ is strictly greater than the slope of $\mathcal{G}^{\prime}$, then $\mathcal{H}_{\mathcal{G}, \mathcal{G}^{\prime}}$ vanishes.

(2) If the slopes of $\mathcal{G}$ and $\mathcal{G}^{\prime}$ are equal, then $\mathcal{H}_{\mathcal{G}, \mathcal{G}^{\prime}}$ is an étale p-divisible group.

(3) If the slope of $\mathcal{G}$ is strictly less than the slope of $\mathcal{G}^{\prime}$, then $\mathcal{H}_{\mathcal{G}, \mathcal{G}^{\prime}}$ is a connected p-divisible group.

Corollary 4.1.11. If $\mathcal{G}$ and $\mathcal{G}^{\prime}$ are isoclinic and the slope of $\mathcal{G}$ is strictly less than the slope of $\mathcal{G}^{\prime}$ and $\mathcal{H}_{\mathcal{G}, \mathcal{G}^{\prime}}$ has dimension $r$, then the sheaf $\mathscr{H}$ om $\left(\mathcal{G}, \mathcal{G}^{\prime}\right)$ is representable by the scheme

$$
\text { Spec } k\left[\left[x_{1}^{1 / p^{\infty}}, \ldots, x_{r}^{1 / p^{\infty}}\right]\right] /\left(x_{1}, \ldots, x_{r}\right) .
$$

Proof. This follows from Proposition 4.1.2 and Corollary 4.1.10, 
4.2. Rapoport-Zink spaces of PEL type. In this section, we introduce the Rapoport-Zink spaces of PEL type that we will consider, and recall some of the results we will need. In close analogy to the EL case treated in SW13, we define a local avatar of the Hodge-Tate period morphism, mapping the infinite-level Rapoport-Zink space to $\mathscr{F} \ell_{G, \mu}$.

We first introduce PEL structures, as in RZ96, with several simplifying assumptions that will be verified in the global case that we want to consider. Fix a finite-dimensional, semisimple algebra $B$ over $\mathbb{Q}_{p}$, endowed with an anti-involution $*$, and a finite left $B$-module $V$ equipped with an alternating bilinear form

$$
(\cdot, \cdot): V \otimes_{\mathbb{Q}_{p}} V \rightarrow \mathbb{Q}_{p}
$$

such that $(b v, w)=\left(v, b^{*} w\right)$ for all $v, w \in V, b \in B$. The data so far define an algebraic group $G$ over $\mathbb{Q}_{p}$, whose values over a $\mathbb{Q}_{p}$-algebra $R$ are

$$
G(R)=\left\{(g, c) \in G L_{B \otimes R}(V \otimes R) \times R^{\times} \mid(g v, g w)=c(v, w)\right\} .
$$

We refer to $c: G \rightarrow \mathbb{G}_{m}$ as the multiplier character of $G$. We make the general assumption that $G$ is connected, which amounts to excluding type D in the classification.

Moreover, we assume that the data are unramified. More precisely, we assume that $B$ is a product of matrix algebras over unramified extensions of $\mathbb{Q}_{p}$, and admits a $*$-stable maximal $\mathbb{Z}_{p}$-order $\mathcal{O}_{B} \subset B$, which we fix. Moreover, we assume that there is an $\mathcal{O}_{B}$-stable lattice $\Lambda \subset V$, which is self-dual under $(\cdot, \cdot)$; again, we fix such a lattice $\Lambda$. These data define a reductive group $G_{\mathbb{Z}_{p}}$ over $\mathbb{Z}_{p}$ via

$$
G(R)=\left\{(g, \lambda) \in G L_{\mathcal{O}_{B} \otimes R}(\Lambda \otimes R) \times R^{\times} \mid(g v, g w)=\lambda(v, w)\right\} .
$$

Now also fix a conjugacy class of cocharacters $\mu: \mathbb{G}_{m} \rightarrow G_{\overline{\mathbb{Q}}_{p}}$ such that in the induced weight decomposition of $V_{\overline{\mathbb{Q}}_{p}}$, only weights 0 and 1 appear,

$$
V_{\overline{\mathbb{Q}}_{p}}=V_{0} \oplus V_{1}
$$

and $\lambda \circ \mu: \mathbb{G}_{m} \rightarrow \mathbb{G}_{m}$ is the identity morphism. This implies, in particular, that the subspaces $V_{0}$ and $V_{1}$ are totally isotropic. We let $E / \mathbb{Q}_{p}$ be the field of definition of $\mu$. Finally, we fix an element $b \in G(L)$, satisfying the compatibility $b \in B\left(G, \mu^{-1}\right)$. Set $\breve{E}:=E \cdot L$.

Note that the condition $b \in B\left(G, \mu^{-1}\right)$ together with the condition on the weights of $\mu$ on $V$ imply that the slopes of $b$ on $V$ are in $[-1,0]$. In particular, in our (nonstandard) normalization of the covariant Dieudonné module, there is a $p$-divisible group $\mathbb{X}_{b}$ over $\overline{\mathbb{F}}_{p}$ whose rational Dieudonné module is given by

$$
\left(V \otimes_{\mathbb{Q}_{p}} L, b(\mathrm{id} \otimes \sigma)\right) ;
$$

then $\mathbb{X}_{b}$ is uniquely determined up to isogeny, and its universal cover $\widetilde{\mathbb{X}}_{b}$ is uniquely determined. By functoriality, $\mathbb{X}_{b}$ is equipped with an action $\iota: B \rightarrow \operatorname{End}\left(\widetilde{\mathbb{X}}_{b}\right)$ and with a symmetric polarization (i.e. an anti-symmetric quasi-isogeny to its dual), with induced Rosati involution being compatible with $*$ on $B$.

Write $\mathcal{D}=(B, *, V,(\cdot, \cdot), b, \mu)$ for the rational data and $\mathcal{D}^{\text {int }}=\left(\mathcal{O}_{B}, *, \Lambda,(\cdot, \cdot), b, \mu\right)$ for the integral data.

Definition 4.2.1. The Rapoport-Zink space $\mathfrak{M}_{\mathcal{D}^{\text {int }}}$ of PEL type associated to $\mathcal{D}^{\text {int }}$ is the functor on $\mathrm{Nilp}_{\mathcal{O}_{\breve{E}}}^{\text {op }}$ sending an $\mathcal{O}_{\breve{E}_{0}}$-algebra $R$ to the set of isomorphism classes of pairs $(\mathcal{G}, \rho)$, where $\mathcal{G}$ is a p-divisible group over $R$ equipped with an action of $\mathcal{O}_{B}$ and a principal polarization whose induced Rosati involution is compatible with 
* on $\mathcal{O}_{B}$, such that the $\mathcal{O}_{B}$-action satisfies the determinant condition (see 3.23 in RZ96] for a precise formulation), and

$$
\rho: \mathbb{X}_{b} \times_{\overline{\mathbb{F}}_{p}} R / p \rightarrow \mathcal{G} \times{ }_{R} R / p
$$

is a quasi-isogeny compatible with the $\mathcal{O}_{B}$-action and the polarization, up to an automorphism of $\widetilde{\mu}_{p^{\infty}, R / p}$.

The following combines Theorem 3.25 and $\S 3.82$ of [RZ96]. In our unramified situation, and excluding type $\mathrm{D}$, we may allow $p=2$.

Theorem 4.2.2. The functor $\mathfrak{M}_{\mathcal{D}^{\text {int }}}$ is representable by a formal scheme which locally admits a finitely generated ideal of definition. Moreover, $\mathfrak{M}_{\mathcal{D}^{\text {int }}}$ is formally smooth.

We let $\mathcal{M}_{\mathcal{D}^{\text {int }}}:=\left(\mathfrak{M}_{\mathcal{D}^{\text {int }}}\right)_{\eta}^{\text {ad }}$ be the adic generic fiber associated to the formal scheme (representing) $\mathfrak{M}_{\mathcal{D}^{\text {int }}}$. The adic generic fiber is taken in the sense of Section 2 of [SW13]: Proposition 2.2.1 of loc. cit. gives a fully faithful functor

$$
\mathfrak{M} \mapsto \mathfrak{M}^{\text {ad }}
$$

from formal schemes over $\mathcal{O}_{\breve{E}}$ which locally admit a finitely generated ideal of definition to adic spaces over $\operatorname{Spa}\left(\mathcal{O}_{\breve{E}}, \mathcal{O}_{\breve{E}}\right)$, and

$$
\mathfrak{M}_{\eta}^{\mathrm{ad}}:=\mathfrak{M}^{\mathrm{ad}} \times_{\operatorname{Spa}\left(\mathcal{O}_{\breve{E}}, \mathcal{O}_{\breve{E}}\right)} \operatorname{Spa}\left(\breve{E}, \mathcal{O}_{\breve{E}}\right) .
$$

Then $\mathcal{M}_{\mathcal{D}^{\text {int }}}$ agrees with the adic space corresponding to the usual rigid-analytic generic fibre of $\mathfrak{M}_{\mathcal{D}^{\text {int }}}$.

For each $n \geq 1$, one can define a cover $\mathcal{M}_{\mathcal{D}^{\text {int }, n}}$ of $\mathcal{M}_{\mathcal{D}^{\text {int }}}$ which parametrizes full level $n$ structures. More precisely, define the compact open subgroups

$$
K_{0}:=\left\{g \in G\left(\mathbb{Q}_{p}\right) \mid g \Lambda=\Lambda\right\}
$$

and

$$
K_{n}:=\left\{g \in K_{0} \mid g \equiv 1 \quad\left(\bmod p^{n}\right)\right\} .
$$

Let $\mathcal{M}_{\mathcal{D}^{\text {int }, n}}$ be the functor on complete affinoid $\left(\breve{E}\left(\zeta_{p^{n}}\right), \mathcal{O}_{\breve{E}\left(\zeta_{p^{n}}\right)}\right)$-algebras parametrizing $\mathcal{O}_{B}$-linear maps

$$
\Lambda / p^{n} \rightarrow \mathcal{G}\left[p^{n}\right]_{\eta}^{\mathrm{ad}}\left(R, R^{+}\right)
$$

which match the pairing $(\cdot, \cdot)$ on $\Lambda$ with the one induced by the polarization on $\mathcal{G}\left[p^{n}\right]$. Here, note that the second pairing takes values in $\mu_{p^{n}}$, but using the fixed primitive $p^{n}$-th root of unity $\zeta_{p^{n}} \in E\left(\zeta_{p^{n}}\right)$, we can identify $\mu_{p^{n}} \cong \mathbb{Z} / p^{n}$. Then by

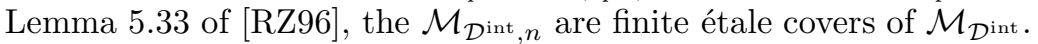

We can also define an infinite-level version of these Rapoport-Zink spaces.

Definition 4.2.3. Let $\mathcal{M}_{\mathcal{D}^{\text {int }}, \infty}$ be the functor on complete affinoid $\left(\breve{E}\left(\zeta_{p^{\infty}}\right), \mathcal{O}_{\breve{E}\left(\zeta_{p} \infty\right)}\right)$ algebras sending $\left(R, R^{+}\right)$to the set of triples $(\mathcal{G}, \rho, \alpha)$, where $(\mathcal{G}, \rho) \in \mathcal{M}_{\mathcal{D}^{\text {int }}}\left(R, R^{+}\right)$ and

$$
\alpha: \Lambda \rightarrow T_{p} \mathcal{G}_{\eta}^{\text {ad }}\left(R, R^{+}\right)
$$

is a morphism of $\mathcal{O}_{B}$-modules such that the following conditions are satisfied. 
(1) The pairing $(\cdot, \cdot)$ on $\Lambda$ matches the pairing on $T_{p} \mathcal{G}$ induced by the polarization. More precisely, the diagram

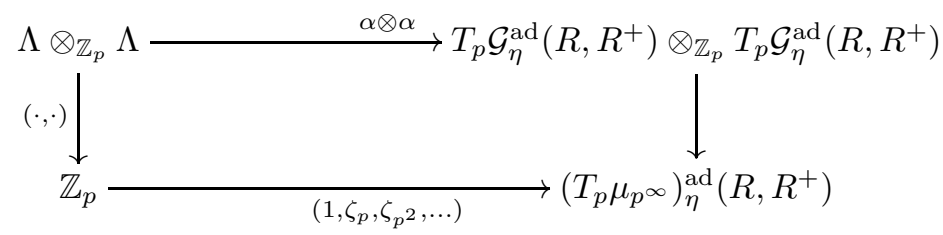

commutes, where the right vertical map is the pairing induced from the polarization, and the lower map is defined using the fixed p-power roots of unity in the base field $E\left(\zeta_{p^{\infty}}\right)$.

(2) The induced maps

$$
\Lambda \rightarrow T_{p} \mathcal{G}_{\eta}^{\mathrm{ad}}\left(C, C^{+}\right)
$$

are isomorphisms, for all geometric points $\mathrm{Spa}\left(C, C^{+}\right)$of $\operatorname{Spa}\left(R, R^{+}\right)$.

Recall that we have the quasi-logarithm map defined in Section 3 of [SW13, which induces a map of sheaves on complete affinoid $\left(\breve{E}, \mathcal{O}_{\breve{E}}\right)$-algebras $\left(R, R^{+}\right)$:

$$
\mathrm{q} \log _{\mathbb{X}_{b}}:\left(\tilde{\mathbb{X}}_{b}\right)_{\eta}^{\mathrm{ad}}\left(R, R^{+}\right) \rightarrow D\left(\mathbb{X}_{b}\right)[1 / p] \otimes_{L} R .
$$

If $\left(R, R^{+}\right)=\left(C, C^{+}\right)$is a geometric point, then the image of $T_{p} \mathcal{G}_{\eta}^{\text {ad }}\left(C, C^{+}\right) \otimes_{\mathbb{Z}_{p}} C$ under q $\log _{\mathbb{X}_{b}}$ can be identified with $\left(\text { Lie } \mathcal{G}^{\vee}\right)^{\vee} \otimes C$.

The arguments in Section 6 of [SW13] give the following theorem. (The case of Rapoport-Zink spaces of EL type is Theorem 6.5.4 of [SW13]. We remark that [SW13] follows the conventions on $b$ and $\mu$ in [RZ96], which differ from our conventions here.)

Theorem 4.2.4. The functor $\mathcal{M}_{\mathcal{D}^{\mathrm{int}}, \infty}$ is representable by an adic space over $\operatorname{Spa}\left(\breve{E}\left(\zeta_{p^{\infty}}\right), \mathcal{O}_{\breve{E}\left(\zeta_{p} \infty\right)}\right)$. The space $\mathcal{M}_{\mathcal{D}^{\text {int }}, \infty}$ is preperfectoid, and

$$
\mathcal{M}_{\mathcal{D}^{\text {int }, \infty}} \sim \underset{n}{\lim _{n}} \mathcal{M}_{\mathcal{D}^{\text {int }, n}}
$$

Moreover, there is the following alternate description of $\mathcal{M}_{\mathcal{D}^{\text {int }}, \infty}$, which depends only on the rational data $\mathcal{D}$. The sheaf $\mathcal{M}_{\mathcal{D}^{\text {int }}, \infty}$ is the sheafification of the functor on complete affinoid $\left(\breve{E}\left(\zeta_{p^{\infty}}, \mathcal{O}_{\breve{E}\left(\zeta_{p} \infty\right)}\right)\right.$-algebras sending $\left(R, R^{+}\right)$to the set of $B$ linear maps

$$
V \rightarrow\left(\tilde{\mathbb{X}}_{b}\right)_{\eta}^{\mathrm{ad}}\left(R, R^{+}\right)
$$

which match the pairing $(\cdot, \cdot)$ on $V$ with the polarization on $\left(\widetilde{\mathbb{X}}_{b}\right)_{\eta}^{\text {ad }}$ (up to the fixed choice of p-power roots of unity, as above) and which in addition satisfy:

(1) The image of $V \otimes_{\mathbb{Q}_{p}} R$ in $D\left(\mathbb{X}_{b}\right)[1 / p] \otimes_{L} R$ is totally isotropic under the pairing $(\cdot, \cdot)$ induced by the identification $D\left(\mathbb{X}_{b}\right)[1 / p] \simeq V \otimes_{\mathbb{Q}_{p}} L$.

(2) The quotient $W$ of $D\left(\mathbb{X}_{b}\right)[1 / p] \otimes_{L} R$ by the image of $V \otimes_{\mathbb{Q}_{p}} R$ is a finite projective $R$-module, which locally on $R$ is isomorphic to $V_{1} \otimes R$ as a $B \otimes_{\mathbb{Q}_{p}}$ $R$-module.

(3) For any point $\mathrm{Spa}\left(C, C^{+}\right)$of $\mathrm{Spa}\left(R, R^{+}\right)$, the sequence

$$
0 \rightarrow V \rightarrow\left(\widetilde{\mathbb{X}}_{b}\right)_{\eta}^{\operatorname{ad}}\left(C, C^{+}\right) \rightarrow W \otimes_{R} C \rightarrow 0
$$

is exact. 
Proof. To see that $\mathcal{M}_{\mathcal{D}^{\text {int }}, \infty}$ is representable by a preperfectoid space, we will show that it is a closed subfunctor of the Rapoport-Zink space at infinite level $\mathcal{M}_{\infty}$ for the $p$-divisible group $\mathbb{X}_{b}$, which is defined in Section 6.3 of [SW13]. Recall that the space $\mathcal{M}_{\infty}$ only keeps track of deformations of $\mathbb{X}_{b}$, without the $\mathcal{O}_{B}$-action or the polarization. By abuse of notation, let us actually denote by $\mathcal{M}_{\infty}$ the base change of this space to $\operatorname{Spa}\left(\breve{E}\left(\zeta_{p^{\infty}}\right), \mathcal{O}_{\breve{E}\left(\zeta_{p} \infty\right)}\right)$.

We claim that the natural (forgetful) map $\mathcal{M}_{\mathcal{D}^{\text {int }}, \infty} \hookrightarrow \mathcal{M}_{\infty}$ is a closed embedding. We follow Theorem 3.25 of [RZ96. Let $\mathcal{G}$ be the universal $p$-divisible group over $\mathcal{M}_{\infty}$. The conditions that the $\mathcal{O}_{B}$-action and the polarization lift to quasi-isogenies on $\mathcal{G}$ depend only on preserving the Hodge filtration on $D(\mathcal{G})[1 / p]$, by Grothendieck-Messing theory, so these are closed conditions. They correspond to restricting to a closed subset of the image of the Grothendieck-Messing period morphism. On the other hand, the condition that a quasi-isogeny be a genuine isogeny on the adic generic fiber is an open and closed condition. (This follows in the same way as Proposition 3.3.3 of [SW13], which is the special case of a quasiisogeny from the $p$-divisible group $\mathbb{Q}_{p} / \mathbb{Z}_{p}$. In the general case, the key observation is that $\{e\} \hookrightarrow \operatorname{Hom}\left(\mathcal{G}_{1}\left[p^{n}\right], \mathcal{G}_{2}\left[p^{n}\right]\right)_{\eta}^{\text {ad }}$ is an open and closed embedding when $\{e\}$ corresponds to the trivial isogeny and $n \in \mathbb{Z}_{\geq 1}$.) Finally, the condition that the trivialization $\alpha$ of $\left(T_{p} \mathcal{G}\right)_{\eta}^{\text {ad }}$ be $\mathcal{O}_{B}$-linear and respect the polarization is closed.

The first part of the theorem now follows from Theorem 6.3.4 of [SW13, which shows that $\mathcal{M}_{\infty}$ is preperfectoid and Proposition 2.3.7 of loc. cit., which shows that a closed subspace of a preperfectoid space is preperfectoid.

For the second part, let $\mathcal{M}_{\mathcal{D}, \infty}$ be the functor defined by the rational data. There is a natural map of functors $\mathcal{M}_{\mathcal{D}^{\text {int }}, \infty} \rightarrow \mathcal{M}_{\mathcal{D}, \infty}$ : For $\left(R, R^{+}\right)$a complete affinoid algebra over $\left(\breve{E}\left(\zeta_{p^{\infty}}\right), \mathcal{O}_{\breve{E}\left(\zeta_{p} \infty\right)}\right)$, let $(\mathcal{G}, \rho, \alpha) \in \mathcal{M}_{\mathcal{D}^{\text {int }}, \infty}\left(R, R^{+}\right)$. The quasi-isogeny $\rho$ gives an identification $\widetilde{\mathbb{X}}_{b} \simeq \widetilde{\mathcal{G}}$. The map from the rational Tate module of $\mathcal{G}$ to its universal cover, precomposed with the trivialization $\alpha$ gives a map

$$
V \rightarrow\left(\widetilde{\mathbb{X}}_{b}\right)_{\eta}^{\text {ad }}\left(R, R^{+}\right)
$$

By construction, this map will respect the polarization and the $B$-action. The first condition is satisfied because the image of $V \otimes_{\mathbb{Q}_{p}} R$ in $D\left(\mathbb{X}_{b}\right)[1 / p] \otimes_{L} R$ can be identified with (Lie $\left.\mathcal{G}^{\vee}\right)^{\vee} \otimes R$ (see the proof of Proposition 7.1.1 of [SW13]). The compatibility between the pairing $(\cdot, \cdot)$ on $V$ and the polarization on $\mathcal{G}$ imply that $\left(\text { Lie } \mathcal{G}^{\vee}\right)^{\vee} \otimes R$ is totally isotropic under $(\cdot, \cdot)$. The second condition is satisfied because $W$ can be identified with Lie $\mathcal{G} \otimes R$. The third condition follows from [SW13, Proposition 3.4.2 (v)].

We also recall the functor $\mathcal{M}_{\infty}^{\prime}$ defined in Section 6.3 of [SW13] (which again, we base change to $\left.\operatorname{Spa}\left(\breve{E}\left(\zeta_{p^{\infty}}\right), \mathcal{O}_{\breve{E}\left(\zeta_{p} \infty\right)}\right)\right)$ : this parametrizes maps

$$
V \rightarrow\left(\widetilde{\mathbb{X}}_{b}\right)_{\eta}^{\mathrm{ad}}\left(R, R^{+}\right)
$$

which satisfy:

(1) the quotient $W$ of $D\left(\mathbb{X}_{b}\right)[1 / p] \otimes_{L} R$ by the image of $V \otimes_{\mathbb{Q}_{p}} R$ is a finite projective $R$-module, of the same rank as that of $V_{1}$.

(2) For any geometric point $\mathrm{Spa}\left(C, C^{+}\right)$of $\mathrm{Spa}\left(R, R^{+}\right)$, the sequence

$$
0 \rightarrow V \rightarrow\left(\widetilde{\mathbb{X}}_{b}\right)_{\eta}^{\operatorname{ad}}\left(C, C^{+}\right) \rightarrow W \otimes_{R} C \rightarrow 0
$$

is exact. 
Lemma 6.3 .6 of $\left[\right.$ SW13] shows that $\mathcal{M}_{\infty} \stackrel{\sim}{\rightarrow} \mathcal{M}_{\infty}^{\prime}$ and we have a commutative diagram of adic spaces

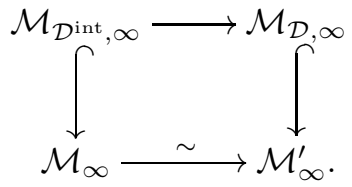

The bottom map is an isomorphism and the vertical maps are closed embeddings.

It remains to see that the top map is surjective. For this, note that there is a p-divisible group $\mathcal{G}$ over $\mathcal{M}_{\mathcal{D}, \infty}$, obtained by restriction from $\mathcal{M}_{\infty}$. The integral Tate module $\left(T_{p} \mathcal{G}\right)_{\eta}^{\text {ad }}$ is identified with the lattice $\Lambda \subset V$, which is stable under $\mathcal{O}_{B}$ and self-dual under $(\cdot, \cdot)$. The $p$-divisible group $\mathcal{G}$ is equipped locally on $\mathcal{M}_{\mathcal{D}, \infty}$ with a quasi-isogeny on the special fiber to $\mathbb{X}_{b}$. The first two conditions on the image of $V \otimes_{\mathbb{Q}_{p}} R$ ensure that the $B$-action and the polarization on $D\left(\mathbb{X}_{b}\right)[1 / p] \otimes_{L} R$ preserve the Hodge filtration of $\mathcal{G}$, so that they define quasi-isogenies on $\mathcal{G}$. The fact that these quasi-isogenies are genuine isogenies follows from the fact that they preserve the integral Tate module.

From now on, we identify $\mathcal{M}_{\mathcal{D}^{\text {int }}, \infty} \simeq \mathcal{M}_{\mathcal{D}, \infty}$, so the moduli problem only depends on the rational data $\mathcal{D}$.

Recall that $\mathscr{F} \ell_{G, \mu}$ is the flag variety over $\operatorname{Spa}\left(E, \mathcal{O}_{E}\right)$ parametrizing filtrations on $\operatorname{Rep} G$ of the same type as the ascending filtration corresponding to the cocharacter $\mu$. On the faithful representation $V$ of $G, \mu$ induces the decomposition

$$
V_{\overline{\mathbb{Q}}_{p}}=V_{0} \oplus V_{1}
$$

and the ascending filtration is given by

$$
\operatorname{Fil}_{-1, \mu}\left(V_{\overline{\mathbb{Q}}_{p}}\right):=V_{1} \text { and } \operatorname{Fil}_{0, \mu}\left(V_{\overline{\mathbb{Q}}_{p}}\right):=V_{\overline{\mathbb{Q}}_{p}} .
$$

In the case we are considering, we can be more explicit: $\mathscr{F} \ell_{G, \mu}$ parametrizes $B$-equivariant quotients $W^{\prime}$ of $V \otimes_{\mathbb{Q}_{p}} R$ that are finite projective $R$-modules such that

(1) the kernel of the map $V \otimes R \rightarrow W^{\prime}$ is totally isotropic under $(\cdot, \cdot)$ and

(2) locally on $R, W^{\prime}$ is isomorphic to $V_{0} \otimes R$ as $B \otimes \mathbb{Q}_{p} R$-modules.

Proposition 4.2.5. There is a local Hodge-Tate period map

$$
\pi_{H T}: \mathcal{M}_{\mathcal{D}, \infty} \rightarrow \mathscr{F} \ell_{G, \mu},
$$

sending an $\left(R, R^{+}\right)$-valued point of $\mathcal{M}_{\mathcal{D}, \infty}$ given by a map $V \rightarrow\left(\widetilde{\mathbb{X}}_{b}\right)_{\eta}{ }_{\eta}\left(R, R^{+}\right)$to the quotient of $V \otimes_{\mathbb{Q}_{p}} R$ given as the image of the map

$$
V \otimes_{\mathbb{Q}_{p}} R \rightarrow D\left(\mathbb{X}_{b}\right)[1 / p] \otimes_{L} R
$$

The local Hodge-Tate period map is $G\left(\mathbb{Q}_{p}\right)$-equivariant.

Proof. This is proved in exactly the same way as Proposition 7.1.1 of SW13.

Recall that, by Theorem 3.5.9, we have a stratification of $\mathscr{F} \ell_{G, \mu}$ by locally closed strata indexed by elements of $B\left(G, \mu^{-1}\right)$ and that we have fixed an element $b \in$ $B\left(G, \mu^{-1}\right)$.

Proposition 4.2.6. The local Hodge-Tate period map factors through

$$
\pi_{H T}^{b}: \mathcal{M}_{\mathcal{D}, \infty} \rightarrow \mathscr{F} \ell_{G, \mu}^{b} .
$$


Proof. It suffices to check this on $\operatorname{Spa}\left(C, \mathcal{O}_{C}\right)$-valued points. Thus, we have a $p$ divisible group $\mathcal{G} / \mathcal{O}_{C}$ with extra structures, equipped with a quasi-isogeny $G \times{ }_{\mathcal{O}_{C}}$ $\mathcal{O}_{C} / p \rightarrow \mathbb{X}_{b} \times_{\overline{\mathbb{F}}_{p}} \mathcal{O}_{C} / p$. Moreover, there is a trivialization $T_{p} \mathcal{G} \otimes_{\mathbb{Z}_{p}} \mathbb{Q}_{p}=V$ compatible with all extra structures, and we have the Hodge-Tate filtration

$$
0 \rightarrow \text { Lie } \mathcal{G} \otimes C(1) \rightarrow T_{p} \mathcal{G} \otimes_{\mathbb{Z}_{p}} C \rightarrow\left(\text { Lie } \mathcal{G}^{\vee}\right)^{\vee} \otimes C \rightarrow 0
$$

where Fil $-1=$ Lie $\mathcal{G} \otimes C(1)$, and $\mathrm{Fil}_{0}=T_{p} \mathcal{G} \otimes_{\mathbb{Z}_{p}} C$.

Let $\mathcal{E}$ be the $G$-bundle on $X_{C^{b}}$ corresponding to the image of $\mathcal{G}$ under $\pi_{H T}$ and the identification $\mathscr{F} \ell_{G, \mu} \cong \mathrm{Gr}_{G, \mu}^{B_{\mathrm{dR}}^{+}}$. Let $\mathcal{E}_{V}$ be the vector bundle on $X_{C^{b}}$ corresponding to $\mathcal{E}$ and the faithful representation $V$; note that $\mu$ is still minuscule as cocharacter into $G L(V)$. Then $\mathcal{E}_{V}$ is constructed from the $\mathbb{B}_{\mathrm{dR}, C}^{+}$-lattice $\Xi$ in $V \otimes_{\mathbb{Q}_{p}} \mathbb{B}_{\mathrm{dR}, C}$ inducing the above filtration on $V \otimes_{\mathbb{Q}_{p}} C$ under the Bialynicki-Birula map. Explicitly, if $\xi \in \mathbb{B}_{\mathrm{dR}, C}^{+}$is a generator of the maximal ideal, then the lattice $\Xi_{x} \subset V \otimes_{\mathbb{Q}_{p}} \mathbb{B}_{\mathrm{dR}, C}$ satisfies

$$
V \otimes_{\mathbb{Q}_{p}} \mathbb{B}_{\mathrm{dR}, C}^{+} \subset \Xi \subset V \otimes_{\mathbb{Q}_{p}} \xi^{-1} \mathbb{B}_{\mathrm{dR}, C}^{+}
$$

and

$$
\Xi /\left(V \otimes_{\mathbb{Q}_{p}} \mathbb{B}_{\mathrm{dR}, C}^{+}\right)=\text {Lie } \mathcal{G} \otimes C .
$$

Then $\mathcal{E}_{V}$ is the modification of the trivial vector bundle $V \otimes_{\mathbb{Q}_{p}} \mathcal{O}_{X_{C^{b}}}$ at the point $\infty$ by the lattice $\Xi$.

In the case of a one-step filtration, one can construct the vector bundle $\mathcal{E}_{V}$ directly: it is the unique vector bundle on $X_{C^{b}}$ which fits into the diagram of coherent sheaves

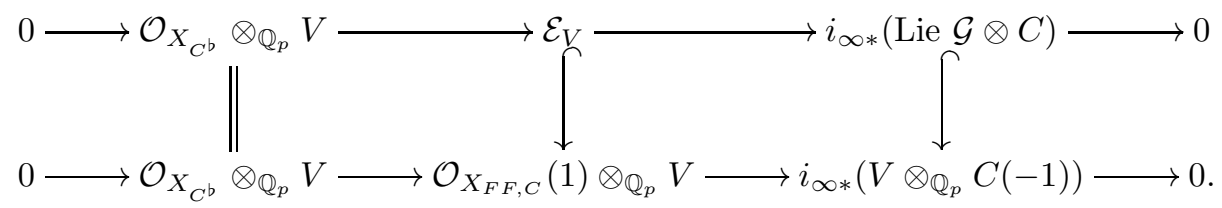

But then the proof of Proposition 5.1.6 of [SW13] shows that $\mathcal{E}_{V}$ is the vector bundle attached to the $p$-divisible group $\mathcal{G} \times \mathcal{O}_{C} \mathcal{O}_{C} / p$, which is quasi-isogenous to $\mathbb{X}_{b} \times_{\overline{\mathbb{F}}_{p}} \mathcal{O}_{C} / p$.

By unraveling the Tannakian formalism behind the construction of the $G$-bundle $\mathcal{E}$ and keeping in mind the fact that $\mathbb{X}_{b}$ together with the $B$-action and polarization determine $b$, we see that $\mathcal{E} \simeq \mathcal{E}_{b}$ as $G$-bundles, as desired.

Remark 4.2.7. The same proof, without keeping track of the polarization, also works in the case of Rapoport-Zink spaces of EL type to show that the local Hodge-Tate period map defined in Proposition 7.1.1 of [SW13] factors through $\mathscr{F} \ell_{G, \mu}^{b}$.

Remark 4.2.8. We have defined the Hodge-Tate filtration in Section 2 in terms of the $p$-adic étale cohomology of a universal family of abelian varieties. If $A / \mathcal{O}_{C}$ is an abelian variety and $\mathcal{G}=A\left[p^{\infty}\right]$, then Proposition 4.15 of [Sch12b] shows that the Hodge-Tate filtration on $T_{p} \mathcal{G} \otimes_{\mathbb{Z}_{p}} C$ is compatible with the filtration defined in Section 2 so the local and global Hodge-Tate period maps are compatible.

Definition 4.2.9. Define the sheaf $\operatorname{Aut}_{G}\left(\widetilde{\mathbb{X}}_{b}\right)$ on $\operatorname{Nilp}_{W\left(\overline{\mathbb{F}}_{p}\right)}^{\mathrm{op}}$ by

$\operatorname{Aut}_{G}\left(\widetilde{\mathbb{X}}_{b}\right)(R)=\left\{\alpha \in \operatorname{Aut}_{B}\left(\widetilde{\mathbb{X}}_{b, R}\right), \beta \in \operatorname{Aut}\left(\widetilde{\mu}_{p \infty}, R\right) \mid \alpha\right.$ respects the polarization up to $\left.\beta\right\}$. 
Lemma 4.2.10. The sheaf $\operatorname{Aut}_{G}\left(\widetilde{\mathbb{X}}_{b}\right)$ is representable by a formal scheme over Spf $W\left(\overline{\mathbb{F}}_{p}\right)$, locally of the form $\operatorname{Spf} W(R)$ for a perfect ring $R$.

Proof. Forgetting all extra structures defines a closed embedding, so it is enough to show representability of Aut $(\widetilde{\mathbb{X}})$ for any $p$-divisible group $\mathbb{X}$ over $\overline{\mathbb{F}}_{p}$. We may assume that $\mathbb{X}$ is completely slope divisible, i.e. that it is isomorphic to a direct sum of slope divisible isoclinic $p$-divisible groups $\mathbb{X}_{i}$, defined over a finite field, for $i=1, \ldots, r$, with non-increasing slopes. Then Aut $(\widetilde{\mathbb{X}})$ is a closed subfunctor of the product of two copies of $\mathscr{H}$ om $\left(\mathbb{X}_{i}, \mathbb{X}_{j}\right)[1 / p]$ over $i, j \in\{1, \ldots, r\}$ with $i \geq j$, via sending an automorphism to the endomorphism, and its inverse. Each of the factors can be identified with the universal cover of the $p$-divisible group $\mathcal{H}_{\mathbb{X}_{i}, \mathbb{X}_{j}}$. Therefore, each of the factors is representable by a formal scheme over $\operatorname{Spf} W\left(\overline{\mathbb{F}}_{p}\right)$, by Proposition 4.1.2.

For the final statement, it is enough to see that $\operatorname{Aut}_{G}\left(\widetilde{\mathbb{X}}_{b}\right)(R)=\operatorname{Aut}_{G}\left(\widetilde{\mathbb{X}}_{b}\right)(R / p)$, and that if $R$ is of characteristic $p$, then Frobenius induces a bijection of $\operatorname{Aut}_{G}\left(\widetilde{\mathbb{X}}_{b}\right)(R)$. Both statements follow from the similar properties of universal covers of $p$-divisible groups, for which see [SW13, Proposition 3.1.3]. by

In fact, one can give a more precise description of $\operatorname{Aut}_{G}\left(\widetilde{\mathbb{X}}_{b}\right)$. As usual, we denote $\rho \in X^{*}(G)_{\operatorname{dom}}$

the half-sum of the positive roots.

Proposition 4.2.11. Let $J_{b}\left(\mathbb{Q}_{p}\right)$ be the locally profinite set $J_{b}\left(\mathbb{Q}_{p}\right)$ made into a formal scheme over $W\left(\overline{\mathbb{F}}_{p}\right)$, i.e. the sections over $U \subset J_{b}\left(\mathbb{Q}_{p}\right)$ are continuous maps $U \rightarrow W\left(\overline{\mathbb{F}}_{p}\right)$. There is a natural map

$$
\operatorname{Aut}_{G}\left(\widetilde{\mathbb{X}}_{b}\right) \rightarrow \underline{J_{b}\left(\mathbb{Q}_{p}\right)}
$$

all of whose fibres are isomorphic to

$$
\operatorname{Spf} W\left(\overline{\mathbb{F}}_{p}\right)\left[\left[x_{1}^{1 / p^{\infty}}, \ldots, x_{d}^{1 / p^{\infty}}\right]\right],
$$

where $d=\left\langle 2 \rho, \nu_{b}\right\rangle$.

Remark 4.2.12. Let us illustrate this result in the case $\mathbb{X}_{b}=\mu_{p \infty} \times \mathbb{Q}_{p} / \mathbb{Z}_{p}$, without extra structures. Then there are no maps $\mu_{p^{\infty}} \rightarrow \mathbb{Q}_{p} / \mathbb{Z}_{p}$, so $\operatorname{Aut}_{G}\left(\widetilde{\mathbb{X}}_{b}\right)$ has lower triangular form; more precisely,

$$
\operatorname{Aut}\left(\widetilde{\mathbb{X}}_{b}\right)=\left(\begin{array}{cc}
\frac{\mathbb{Q}_{p}^{\times}}{\widetilde{\mu_{p^{\infty}}}} & 0 \\
\underline{\mathbb{Q}_{p}^{\times}}
\end{array}\right) .
$$

In this case, $J_{b}\left(\mathbb{Q}_{p}\right)=\mathbb{Q}_{p}^{\times} \times \mathbb{Q}_{p}^{\times}$, and the projection

$$
\operatorname{Aut}_{G}\left(\widetilde{\mathbb{X}}_{b}\right) \rightarrow \underline{J_{b}\left(\mathbb{Q}_{p}\right)}
$$

is given by the diagonal elements. The fibres are given by the unipotent part $\widetilde{\mu_{p^{\infty}}} \cong \operatorname{Spf} W\left(\overline{\mathbb{F}}_{p}\right)\left[\left[x^{1 / p^{\infty}}\right]\right]$.

Proof. It is enough to prove the results for $\operatorname{Aut}_{G}\left(\widetilde{\mathbb{X}}_{b}\right)$ as a formal scheme over $\overline{\mathbb{F}}_{p}$, as all structures lift uniquely to $W\left(\overline{\mathbb{F}}_{p}\right)$ by rigidity of perfect rings. We first consider the case when $\mathbb{X}_{b}$ has an unramified EL structure. By standard Morita arguments, one can reduce to the case when the EL structure is given by $\left(F, \mathcal{O}_{F}\right)$, with $F / \mathbb{Q}_{p}$ an unramified extension and $G=\operatorname{Res}_{F / \mathbb{Q}_{p}} G L_{n}$. If $\left(B, \mathcal{O}_{B}\right)$ is an unramified PEL 
datum and $B=\prod_{i} B_{i}$ is its decomposition into simple factors, then $\mathbb{X}_{b}$ decomposes as $\prod_{i} \mathbb{X}_{b, i}$ and $\operatorname{Aut}_{G}\left(\widetilde{\mathbb{X}}_{b}\right)=\prod_{i} \operatorname{Aut}_{G_{i}}\left(\widetilde{\mathbb{X}}_{b, i}\right)$. Similarly, when $B \simeq M_{d}(F)$ is simple, the equivalence of categories between $p$-divisible groups with $\left(B, \mathcal{O}_{B}\right)$-EL structure and $p$-divisible groups with $\left(F, \mathcal{O}_{F}\right)$-structure means that it suffices to compute $\operatorname{Aut}_{F}\left(\widetilde{\mathbb{X}}_{b}\right)$. See [Ham, Section 4.1] for more details on this reduction step.

If $F=\mathbb{Q}_{p}$, then $G=G L_{n}$ and we are considering quasi-self-isogenies of $p$ divisible groups, without any extra compatibilities. Since $\mathbb{X}_{b}$ is completely slope divisible, we can write it as $\mathbb{X}_{b}=\oplus_{i=1}^{r} \mathbb{X}_{i}$, where the $\mathbb{X}_{i}$ are isoclinic $p$-divisible groups of strictly decreasing slopes $\lambda_{i} \in[0,1]$. Using Corollary 4.1.10 (1), we see that $\operatorname{Aut}\left(\widetilde{\mathbb{X}}_{b}\right)$ takes the lower triangular form

$$
\operatorname{Aut}\left(\widetilde{\mathbb{X}}_{b}\right)=\left(\begin{array}{cccc}
\operatorname{Aut}\left(\widetilde{\mathbb{X}_{1}}\right) & & & \\
\widetilde{\mathcal{H}}_{\mathbb{X}_{2}, \mathbb{X}_{1}} & \operatorname{Aut}\left(\widetilde{\mathbb{X}_{2}}\right) & & \\
\vdots & \vdots & \ddots & \\
\widetilde{\mathcal{H}}_{\mathbb{X}_{r}, \mathbb{X}_{1}} & \widetilde{\mathcal{H}}_{\mathbb{X}_{r}, \mathbb{X}_{2}} & \cdots & \operatorname{Aut}\left(\widetilde{\mathbb{X}_{r}}\right)
\end{array}\right)
$$

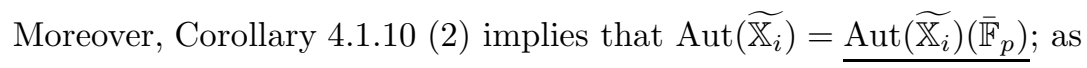

$$
J_{b}\left(\mathbb{Q}_{p}\right)=\operatorname{Aut}\left(\widetilde{\mathbb{X}}_{b}\right)\left(\overline{\mathbb{F}}_{p}\right)=\prod_{i=1}^{r} \operatorname{Aut}\left(\widetilde{\mathbb{X}}_{i}\right)\left(\overline{\mathbb{F}}_{p}\right),
$$

we see that projection to the diagonal defines a map

$$
\operatorname{Aut}\left(\widetilde{\mathbb{X}}_{b}\right) \rightarrow \underline{J_{b}\left(\mathbb{Q}_{p}\right)} .
$$

The structure of the fibres now follows from Corollary 4.1.10 (3) and Proposition 4.1.2 (3). To check that $d=\left\langle 2 \rho, \nu_{b}\right\rangle$, we count dimensions. More precisely, for $i>j, \widetilde{\mathcal{H}}_{\mathbb{X}_{i}, \mathbb{X}_{j}}$ is representable by Spf $\overline{\mathbb{F}}_{p}\left[\left[x_{1}^{1 / p^{\infty}}, \ldots, x_{d_{i, j}}^{1 / p^{\infty}}\right]\right]$, where $d_{i, j}$ is the dimension of $\mathcal{H}_{\mathbb{X}_{i}, \mathbb{X}_{j}}$. If the height of $\mathbb{X}_{i}$ is $m_{i}$, then Lemma 4.1 .8 implies that the slope of $\mathcal{H}_{\mathbb{X}_{i}, \mathbb{X}_{j}}$ is $\lambda_{j}-\lambda_{i}$ and its dimension is $d_{i, j}=m_{i} m_{j}\left(\lambda_{j}-\lambda_{i}\right)$.

On the other hand, by making the root data of $G L_{n}$ explicit, cf. Ham, Appendix $\mathrm{A}]$, we can compute the contribution of the slopes $\lambda_{i}, \lambda_{j}$ to $\left\langle 2 \rho, \nu_{b}\right\rangle$. The positive roots of $G L_{n}$ (corresponding to the Borel subgroup given by the upper triangular matrices) are

$$
R^{+}=\left\{e_{k}-e_{l} \mid k, l \in\{1, \ldots, n\}, k<l\right\} .
$$

We also have

$$
\nu_{b}=(\underbrace{\lambda_{1}, \ldots, \lambda_{1}}_{m_{1}}, \ldots, \underbrace{\lambda_{r}, \ldots, \lambda_{r}}_{m_{r}}) .
$$

The contribution coming from $\lambda_{i}, \lambda_{j}$ to $\left\langle 2 \rho, \nu_{b}\right\rangle$ is precisely $m_{i} m_{j}\left(\lambda_{j}-\lambda_{i}\right)=d_{i, j}$.

The case of a general unramified extension $F / \mathbb{Q}_{p}$ follows in the same way, by working in the category of $p$-divisible groups with $\mathcal{O}_{F}$-action instead. Let $d=[F$ : $\left.\mathbb{Q}_{p}\right]$. The theory developed in Section 4.1 can be extended to define an internal homomorphism in the category of $p$-divisible groups with $\mathcal{O}_{F}$-action. If $\mathcal{G}$ is a $p$ divisible group with $\mathcal{O}_{F}$-action, its rational Dieudonné module $\mathbb{D}(\mathcal{G})$ decomposes as $\mathbb{D}(\mathcal{G})=\oplus_{\tau: \mathcal{O}_{F} \hookrightarrow W\left(\overline{\mathbb{F}}_{p}\right)} \mathbb{D}(\mathcal{G})_{\tau}$. Choose an embedding $\tau_{0}: \mathcal{O}_{F} \hookrightarrow W\left(\overline{\mathbb{F}}_{p}\right)$ and let $\mathbb{D}_{F}(\mathcal{G}):=\mathbb{D}(\mathcal{G})_{\tau_{0}}$. The analogue of Lemma 4.1.8 holds for $\mathbb{D}_{F}$ and homomorphisms of $p$-divisible groups with $\mathcal{O}_{F}$-action, with the same proof (but replacing $\varphi$ by 
$\varphi^{d}$ and embedding $F$ into $B_{\text {cris }}^{+}$via $\left.\tau_{0}\right) 19$ The structure of $\operatorname{Aut}_{F}\left(\widetilde{\mathbb{X}}_{b}\right)$ can now be deduced in the same way. The dimension computation is also analogous to the one above. Let $\mathbb{X}_{b}=\oplus_{i=1}^{r} \mathbb{X}_{i}$, with the slope of the $F$-isocrystal attached to $\mathbb{X}_{i}$ being equal to $\lambda_{i}$ (here, $0 \leq \lambda_{i} \leq d$, and $\lambda_{i} / d$ is the slope of $\mathbb{X}_{i}$ as a $p$ divisible group) and $\mathbb{X}_{i}$ having height $m_{i}$ as a $p$-divisible group with $\mathcal{O}_{F}$-action, i.e. height $d m_{i}$ as $p$-divisible group. The dimension of the $p$-divisible group with $\mathcal{O}_{F}$-action corresponding to the $\mathcal{O}_{F}$-linear homomorphisms between $\mathbb{X}_{i}$ and $\mathbb{X}_{j}$ is $d_{i, j}=m_{i} m_{j}\left(\lambda_{j}-\lambda_{i}\right)$. On the other hand, the positive roots of $\operatorname{Res}_{F / \mathbb{Q}_{p}} G L_{n}$ are

$$
R^{+}=\left\{e_{\tau, k}-e_{\tau, l} \mid k, l \in\{1, \ldots, n\}, k<l, \tau: F \hookrightarrow \overline{\mathbb{Q}}_{p}\right\}
$$

and,

$$
\nu_{b}=(\underbrace{\frac{\lambda_{1}}{d}, \ldots, \frac{\lambda_{1}}{d}}_{m_{1}}, \ldots, \underbrace{\frac{\lambda_{r}}{d}, \ldots, \frac{\lambda_{r}}{d}}_{m_{r}}) .
$$

The contribution from slopes $\lambda_{i}, \lambda_{j}$ is again $d_{i, j}=m_{i} m_{j}\left(\lambda_{j}-\lambda_{i}\right)$.

We now consider the case when $\mathbb{X}_{b}$ has an unramified PEL structure. Recall that we are assuming that the PEL datum is of type (AC). By similar Moritatheoretic arguments as above, cf. [Ham, Corollary 4.5], we can write $\left(B, \mathcal{O}_{B}, *\right)=$ $\prod_{i}\left(B_{i}, \mathcal{O}_{B_{i}}, *\right)$ as a product of simple PEL data. On the level of quasi-self-isogenies we get

$$
\operatorname{Aut}_{G}\left(\widetilde{\mathbb{X}}_{b}\right)=\left(\prod_{i} \operatorname{Aut}_{G_{i}}\left(\widetilde{\mathbb{X}}_{b, i}\right)\right)^{1} \hookrightarrow \prod_{i} \operatorname{Aut}_{G_{i}}\left(\widetilde{\mathbb{X}}_{b, i}\right),
$$

where $\left(\prod_{i} \operatorname{Aut}_{G_{i}}\left(\widetilde{\mathbb{X}}_{b, i}\right)\right)^{1}$ is a closed subfunctor of the product, defined by the condition that the similitude factors on each term are the same. The group $G$ is defined similarly, as the closed subgroup $\left(\prod_{i} G_{i}\right)^{1} \hookrightarrow \prod_{i} G_{i}$. The similitude factor on $\operatorname{Aut}_{G_{i}}\left(\widetilde{\mathbb{X}}_{b_{i}}\right)$ defines a map

$$
\operatorname{Aut}_{G_{i}}\left(\widetilde{\mathbb{X}}_{b_{i}}\right) \rightarrow \underline{\mathbb{Q}_{p}^{\times}}
$$

which will factor as

$$
\operatorname{Aut}_{G_{i}}\left(\widetilde{\mathbb{X}}_{b_{i}}\right) \rightarrow \underline{J_{b_{i}}\left(\mathbb{Q}_{p}\right)} \rightarrow \underline{\mathbb{Q}_{p}^{\times}},
$$

where the latter map is the natural similitude morphism on $J_{b_{i}}$. We see that the result for all $G_{i}$ implies the result for $G$, so we can assume that $G$ is simple.

We reduce to one of the following three cases.

(1) $\mathbb{X}_{b}$ is a $p$-divisible group with $\left(F, \mathcal{O}_{F}\right)$-EL structure, where $F / \mathbb{Q}_{p}$ is unramified.

(2) $\mathbb{X}_{b}$ is a $p$-divisible group with $\left(F, \mathcal{O}_{F}, *\right)$-PEL structure, where $*$ is the identity on $F$.

(3) $\mathbb{X}_{b}$ is a $p$-divisible group with $\left(F, \mathcal{O}_{F}, *\right)$-PEL structure, with $\mathbb{Q}_{p} \subset F^{+} \subset F$ unramified extensions, $*$ an automorphism of order 2 and $F^{+}=F^{*=1}$.

\footnotetext{
${ }^{19}$ For $p$-divisible groups with $\mathcal{O}_{F}$-action, there is a more restricted notion of $p$-divisible $\mathcal{O}_{F^{-}}$ module; the requirement is that the two actions of $\mathcal{O}_{F}$ on the Lie algebra agree. This condition cannot be formulated for $p$-divisible groups with $\mathcal{O}_{F}$-action up to quasi-isogeny, and in fact for $p$-divisible groups with $\mathcal{O}_{F}$-action up to quasi-isogeny, everything works very similarly to the case of $p$-divisible $\mathcal{O}_{F}$-modules. For example, note that $B\left(F, G L_{n}\right)=B\left(\mathbb{Q}_{p}, \operatorname{Res}_{F / \mathbb{Q}_{p}} G L_{n}\right)$.
} 
The first case was already dealt with above. The second case corresponds to $G=$ $G S p_{n} / \mathcal{O}_{F}$ with $n$ even, while the third to $G=G U_{n} / \mathcal{O}_{F^{+}}$.

We explain the computation of $\operatorname{Aut}_{G}\left(\widetilde{\mathbb{X}}_{b}\right)$ in the case of $G=G S p_{n} / \mathcal{O}_{F}$. As before $d=\left[F: \mathbb{Q}_{p}\right]$, and we write $\mathbb{X}_{b}=\oplus_{i=1}^{r} \mathbb{X}_{i}$, with each $\mathbb{X}_{i}$ isoclinic of slope $\lambda_{i} \in[0, d]$ as $p$-divisible group with $\mathcal{O}_{F}$-action, and the $\lambda_{i}$ in strictly decreasing order. The fact that $\mathbb{X}_{b}$ is equipped with a symmetric polarization means that $d-\lambda_{i}$ is also a slope of $\mathbb{X}_{b}$, occuring corresponding to the same height $m_{i}$ as $\lambda_{i}$. As before, the restriction of an automorphism of $\widetilde{\mathbb{X}}_{b}$ to the graded pieces $\widetilde{\mathbb{X}}_{i}$ of the slope filtration defines the map

$$
\operatorname{Aut}_{G}\left(\widetilde{\mathbb{X}}_{b}\right) \rightarrow \underline{J_{b}\left(\mathbb{Q}_{p}\right)} .
$$

The fibres of this map can be computed at the same time as the dimension, and we concentrate on the dimension in the following. We can write

$$
\nu_{b}=(\underbrace{\frac{\lambda_{1}}{d}, \ldots, \frac{\lambda_{1}}{d}}_{m_{1}}, \ldots, \underbrace{\frac{\lambda_{r}}{d}, \ldots, \frac{\lambda_{r}}{d}}_{m_{r}}),
$$

with $\lambda_{i}+\lambda_{r+1-i}=d, m_{i}=m_{r+1-i}$. Using the same choices as in Ham, Appendix A] and recalling that $c: G \rightarrow \mathbb{G}_{m}$ is the multiplier character, the positive roots of $G=G S p_{n} / \mathcal{O}_{F}$ are

$$
\begin{gathered}
R^{+}=\left\{e_{\tau, k}-e_{\tau, l} \mid k<l \in\{1, \ldots, n / 2\}, \tau: F \hookrightarrow \overline{\mathbb{Q}}_{p}\right\} \\
\cup\left\{e_{\tau, k}+e_{\tau, l}-c \mid k \neq l \in\{1, \ldots, n / 2\}, \tau: F \hookrightarrow \overline{\mathbb{Q}}_{p}\right\} \\
\cup\left\{2 e_{\tau, k}-c \mid k \in\{1, \ldots, n / 2\}, \tau: F \hookrightarrow \overline{\mathbb{Q}}_{p}\right\} .
\end{gathered}
$$

We compute the contributions coming from slopes $\lambda_{i}, \lambda_{j}$ to both the dimension of $\operatorname{Aut}_{G}\left(\widetilde{\mathbb{X}}_{b}\right)$ and to $\left\langle 2 \rho, \nu_{b}\right\rangle$ and check that they are the same.

(1) If $\lambda_{j}>\lambda_{i} \geq \frac{d}{2}$, then the contribution to the dimension of $\operatorname{Aut}_{G}\left(\widetilde{\mathbb{X}}_{b}\right)$ is, just like in the EL case, $d_{i, j}=m_{i} m_{j}\left(\lambda_{j}-\lambda_{i}\right)$ and it matches the contribution from $\frac{\lambda_{j}}{d}, \frac{\lambda_{i}}{d}$ to $\left\langle 2 \rho, \nu_{b}\right\rangle$ by the same argument. Using the polarization, this also takes care of all cases with $\frac{d}{2} \geq \lambda_{j}>\lambda_{i}$.

(2) If $\lambda_{j} \geq \frac{d}{2} \geq d-\lambda_{i}$, with $i \neq j$, then the contribution to the dimension of $\operatorname{Aut}_{G}\left(\widetilde{\mathbb{X}}_{b}\right)$ is $m_{i} m_{j}\left(\lambda_{i}+\lambda_{j}-d\right)$. This is given by the dimension of the internal Hom $\mathcal{O}_{F}$-module between $\mathbb{X}_{i}^{\vee}$ and $\mathbb{X}_{j}$ if $j<i$, computed as in the EL case, which by the compatibility with the polarization also pins down the quasi-isogeny between $\mathbb{X}_{j}^{\vee}$ and $\mathbb{X}_{i}$. This matches the contribution from $\frac{\lambda_{j}}{d}, 1-\frac{\lambda_{i}}{d}$ and $\frac{\lambda_{i}}{d}, 1-\frac{\lambda_{j}}{d}$ to $\left\langle 2 \rho, \nu_{b}\right\rangle$, using the fact that $\left\langle c, \nu_{b}\right\rangle=1$.

(3) If $\lambda_{i}>\frac{d}{2}$, the contribution to $\left\langle 2 \rho, \nu_{b}\right\rangle$ from $\frac{\lambda_{i}}{d}, 1-\frac{\lambda_{i}}{d}$ is $\frac{m_{i}\left(m_{i}+1\right)}{2}\left(2 \lambda_{i}-\right.$ $d)$. This is also the dimension of the part of $\mathscr{H}_{0 m_{\mathcal{O}_{F}}}\left(\mathbb{X}_{i}^{\vee}, \mathbb{X}_{i}\right)[1 / p]$ which is compatible with the polarization. Indeed, the polarization induces an involution on $\mathscr{H}_{o m_{\mathcal{O}_{F}}}\left(\mathbb{X}_{i}^{\vee}, \mathbb{X}_{i}\right)[1 / p]$ and we can compute the dimension of the part fixed under the polarization using Lemma 4.1.8 the slope is $2 \frac{\lambda_{i}}{d}-1$ and the height of the fixed part as a $p$-divisible $\mathcal{O}_{F}$-module is $\frac{m_{i}\left(m_{i}+1\right)}{2}$.

The case $G=G U_{n}$ is similar and left as an exercise.

Remark 4.2.13. In view of the theory developed in Subsection 4.3 and Corollary 4.3.9 in particular, the dimension of $\operatorname{Aut}_{G}\left(\widetilde{\mathbb{X}}_{b}\right)$ should match the dimension of central leaves inside the Newton stratum corresponding to $b$ on the special fiber 
of a corresponding Shimura variety. This indeed agrees with the dimension of central leaves as computed by [Ham, Corollary 7.8].

Note that there is an action of $\operatorname{Aut}_{G}\left(\widetilde{\mathbb{X}}_{b}\right)$ on $\mathfrak{M}_{\mathcal{D}_{\text {int }}}$. We let $\operatorname{Aut}_{G}\left(\widetilde{\mathbb{X}}_{b}\right)_{\eta}^{\text {ad }}$ be its adic generic fiber over $\operatorname{Spa}\left(L, \mathcal{O}_{L}\right)$. Then the action of $\operatorname{Aut}_{G}\left(\widetilde{\mathbb{X}}_{b}\right)_{\eta}^{\text {ad }}$ on $\mathcal{M}_{\mathcal{D}_{\text {int }}}$ extends to an action on $\mathcal{M}_{\mathcal{D}, \infty}$. The map $\pi_{H T}^{b}: \mathcal{M}_{\mathcal{D}, \infty} \rightarrow \mathscr{F}_{G, \mu}^{b}$ is equivariant for this action with respect to the trivial action on the target. We would like to say that $\pi_{H T}^{b}: \mathcal{M}_{\mathcal{D}, \infty} \rightarrow \mathscr{F}_{G, \mu}^{b}$ is an $\operatorname{Aut}_{G}\left(\widetilde{\mathbb{X}}_{b}\right)_{\eta}^{\text {ad }}$-torsor. However, we have only defined the target as a locally closed subspace of $\mathscr{F} \ell_{G, \mu}$. Also, the condition of being a torsor includes the condition that the map is surjective locally in some specified topology. It is probably necessary to use some of the fine topologies from Wei14 here. Thus, we content ourselves with some more basic information. Recall that $\mathcal{M}_{\mathcal{D}, \infty}$ is preperfectoid and lives over the perfectoid field $E\left(\zeta_{p^{\infty}}\right)^{\wedge}$; thus, one can form a perfectoid space $\widehat{\mathcal{M}}_{\mathcal{D}, \infty}$ as in [SW13, Proposition 2.3.6]. The product

$$
\widehat{\mathcal{M}}_{\mathcal{D}, \infty} \times_{\mathrm{Spa}\left(L, \mathcal{O}_{L}\right)} \operatorname{Aut}_{G}\left(\widetilde{\mathbb{X}}_{b}\right)_{\eta}^{\mathrm{ad}}
$$

exists in the category of adic spaces, and is still a perfectoid space, by the local structure of the automorphism scheme. On the other hand, the space

$$
\mathcal{M}_{\mathcal{D}, \infty} \times \mathscr{F} \ell_{G, \mu} \mathcal{M}_{\mathcal{D}, \infty} \subset \mathcal{M}_{\mathcal{D}, \infty} \times_{\operatorname{Spa}\left(\breve{E}, \mathcal{O}_{\breve{E}}\right)} \mathcal{M}_{\mathcal{D}, \infty}
$$

is preperfectoid (as this condition passes to closed subsets, cf. [SW13, Proposition 2.3.7]), so again we can pass to a perfectoid space

$$
\left(\mathcal{M}_{\mathcal{D}, \infty} \times \mathscr{F} \ell_{G, \mu} \mathcal{M}_{\mathcal{D}, \infty}\right)^{\wedge} .
$$

Proposition 4.2.14. The action map

$$
\widehat{\mathcal{M}}_{\mathcal{D}, \infty} \times_{\operatorname{Spa}\left(L, \mathcal{O}_{L}\right)} \operatorname{Aut}_{G}\left(\widetilde{\mathbb{X}}_{b}\right)_{\eta}^{\text {ad }} \rightarrow\left(\mathcal{M}_{\mathcal{D}, \infty} \times \mathscr{F}_{G, \mu} \mathcal{M}_{\mathcal{D}, \infty}\right)^{\wedge}
$$

is an isomorphism of perfectoid spaces.

Proof. Let $\left(R, R^{+}\right)$be a perfectoid affinoid algebra over $\breve{E} 20$ We have to construct an inverse map

$$
\left(\mathcal{M}_{\mathcal{D}, \infty} \times{\mathscr{F} \ell_{G}, \mu}_{\mathcal{D}, \infty}\right)\left(R, R^{+}\right) \rightarrow\left(\mathcal{M}_{\mathcal{D}, \infty} \times_{\mathrm{Spa}\left(L, \mathcal{O}_{L}\right)} \operatorname{Aut}_{G}\left(\widetilde{\mathbb{X}}_{b}\right)_{\eta}^{\mathrm{ad}}\right)\left(R, R^{+}\right)
$$

Given an element of the source, we have (after localization on $\operatorname{Spa}\left(R, R^{+}\right)$) two $p$ divisible groups $\mathcal{G}_{1}, \mathcal{G}_{2}$ over $R^{+} 21$ equipped with quasi-isogenies to $\mathbb{X}_{b}$ over $R^{+} / p$, and trivializations of the Tate module on the generic fibre. In particular, we get an isomorphism of the $\mathbb{Z}_{p}$-local systems given by the Tate modules of $\mathcal{G}_{1}$ and $\mathcal{G}_{2}$ over $R$, in other words an isomorphism $\mathcal{G}_{1, R} \cong \mathcal{G}_{2, R}$. We need to check that this isomorphism extends to $R^{+}$, as one can then compose this isomorphism with the given quasi-isogenies to $\mathbb{X}_{b}$ over $R^{+} / p$ to get a self-quasi-isogeny of $\mathbb{X}_{b}$, as desired. In this regard, we observe the following lemma, which is a non-noetherian version of a result of Berthelot, Ber80.

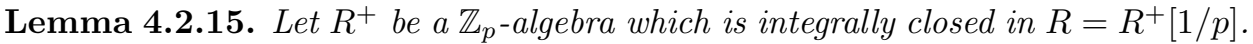
Let $G, H$ be p-divisible groups over $R^{+}$. Assume that the Newton polygon of $G_{s}$ is independent of $s \in \operatorname{Spec}\left(R^{+} / p\right)$, and that the same holds true for $H$. Let $f_{R}$ : $G_{R} \rightarrow H_{R}$ be a morphism of p-divisible groups over $R$. Then $f_{R}$ extends, necessarily

\footnotetext{
${ }^{20}$ In the proof, we are really only using that $R^{+} \subset R$ is bounded, and that this property passes to rational subsets.

${ }^{21}$ Here, we use that $R^{+} \subset R$ is bounded.
} 
uniquely, to a morphism $f: G \rightarrow H$ of p-divisible groups over $R^{+}$if and only if for all geometric rank 1 points $\operatorname{Spa}\left(C, \mathcal{O}_{C}\right)$ of $\operatorname{Spa}\left(R, R^{+}\right)$, the base change $f_{C}: G_{C} \rightarrow$ $H_{C}$ extends to a map $f_{\mathcal{O}_{C}}: G_{\mathcal{O}_{C}} \rightarrow H_{\mathcal{O}_{C}}$.

Proof. For each $n \geq 1$, we have to check that the map $G\left[p^{n}\right]_{R} \rightarrow H\left[p^{n}\right]_{R}$ extends to $R^{+}$. Both schemes $G\left[p^{n}\right], H\left[p^{n}\right]$ in question are affine, and finite locally free over $R^{+}$. Thus, the question whether this morphism extends is the question whether a matrix with entries in $R$ has entries in $R^{+}$. As

$$
R^{+}=\left\{f \in R\left|\forall x \in \operatorname{Spa}\left(R, R^{+}\right):\right| f(x) \mid \leq 1\right\},
$$

we can reduce to the case of a point, i.e. $R=K$ is a complete nonarchimedean field, and $K^{+} \subset K$ is an open and bounded valuation subring. We may also assume that $K$ is algebraically closed, and rename $C=K, C^{+}=K^{+}$. By assumption, the map extends to $\mathcal{O}_{C}$. Let $\mathfrak{m}_{\mathcal{O}_{C}} \subset \mathcal{O}_{C}$ be the maximal ideal; it is also contained in $C^{+}$. Then $C^{+} / \mathfrak{m}_{\mathcal{O}_{C}} \subset \mathcal{O}_{C} / \mathfrak{m}_{\mathcal{O}_{C}}$ is a valuation subring. Finally, we are reduced to the following lemma.

Lemma 4.2.16. Let $V$ be a valuation ring of characteristic $p$ with quotient field $K$. Let $G, H$ be p-divisible groups over $V$ with constant Newton polygon. Then the map

$$
\operatorname{Hom}(G, H) \rightarrow \operatorname{Hom}\left(G_{K}, H_{K}\right)
$$

is a bijection.

Remark 4.2.17. Using this lemma, one can remove the noetherian hypothesis from the main result of [Ber80, i.e. the same fully faithfulness result holds true for any integral domain $R$ in place of $V$. Indeed, to check whether a homomorphism over $K$ extends to $R$, one has to check whether certain matrices over $K$ have entries in $R$, which can be checked on valuation rings.

Proof. The map is clearly injective. For surjectivity, we have to check as above that certain matrices with coefficients in $K$ have entries in $V$. Thus, we may assume that $K$ is algebraically closed.

Observe that it is enough to prove the result up to quasi-isogeny. Indeed, if $f: G \rightarrow H$ becomes divisible by $p$ over $K$, then $G[p]_{K} \subset G_{K}$ is killed by $f$, whence its flat closure $G[p] \subset G$ is killed by $f$, which shows that $f$ is divisible by $p$.

Now, e.g. by the Dieudonné-Manin classification, both $G_{K}$ and $H_{K}$ admit a quasi-isogeny to a completely slope divisible $p$-divisible group $G_{0}, H_{0}$ (defined over $\left.\overline{\mathbb{F}}_{p} \subset V\right)$. We may assume that these quasi-isogenies are genuine isogenies; then we may take their flat closures over $V$ and divide $G$, resp. $H$, by them; thus, we may assume that $G_{K}$ and $H_{K}$ are completely slope divisible. Then by OZ02, Proposition 2.3], $G$ and $H$ are themselves completely slope divisible. As $V$ is perfect, both $G$ and $H$ decompose as a direct sum of their isoclinic pieces, cf. OZ02, Proposition 1.3]; thus $G \cong G_{0} \times_{\overline{\mathbb{F}}_{p}} V, H \cong H_{0} \times_{\overline{\mathbb{F}}_{p}} V$.

Finally, we use that the Dieudonné module functor on $V$ is fully faithful, cf. Ber80. Thus, as $G$ and $H$ come via base extension from $\overline{\mathbb{F}}_{p}$, it remains to show that if $(D, \varphi)$ is any isocrystal over $\overline{\mathbb{F}}_{p}$, then

$$
\left(D \otimes_{W\left(\overline{\mathbb{F}}_{p}\right)[1 / p]} W(V)[1 / p]\right)^{\varphi=1}=\left(D \otimes_{W\left(\overline{\mathbb{F}}_{p}\right)[1 / p]} W(K)[1 / p]\right)^{\varphi=1} .
$$


We may assume that $D=D_{\lambda}$ is simple of slope $\lambda=s / r$. In that case, we have to prove

$$
W(V)[1 / p]^{\varphi^{r}=p^{s}}=W(K)[1 / p]^{\varphi^{r}=p^{s}} .
$$

Clearly, the left-hand side is contained in the right-hand side. If $s \neq 0$, then the right-hand is 0 , as follows by looking at the $p$-adic valuation of any nonzero element. We are left with the case $s=0$, where $r=1$. But $W(K)[1 / p]^{\varphi=1}=\mathbb{Q}_{p} \subset$ $W(V)[1 / p]^{\varphi=1}$, finishing the proof.

Using Lemma 4.2.15, we only have to check the result on geometric rank 1 points. But now, by [SW13, Theorem B], $p$-divisible groups over $\mathcal{O}_{C}$ are equivalent to pairs $(T, W)$, where $T$ is a finite free $\mathbb{Z}_{p}$-module, and $W \subset T \otimes_{\mathbb{Z}_{p}} C$ is the Hodge-Tate filtration. Thus, it remains to check that the Hodge-Tate filtration is preserved, but this is true as we started with an element of the fibre product

$$
\left(\mathcal{M}_{\mathcal{D}, \infty} \times \mathscr{F} \ell_{G, \mu} \mathcal{M}_{\mathcal{D}, \infty}\right)\left(R, R^{+}\right) .
$$

We also have the following surjectivity result.

Lemma 4.2.18. Let $C / \breve{E}\left(\zeta_{p^{\infty}}\right)$ be a complete algebraically closed extension with ring of integers $\mathcal{O}_{C}$. Then the map

$$
\pi_{H T}^{b}: \mathcal{M}_{\mathcal{D}, \infty}\left(C, \mathcal{O}_{C}\right) \rightarrow \mathscr{F} \ell_{G, \mu}^{b}\left(C, \mathcal{O}_{C}\right)
$$

is surjective.

Proof. Given $x \in \mathscr{F} \ell_{G, \mu}^{b}\left(C, \mathcal{O}_{C}\right)$, we get (corresponding to the representation $G \rightarrow$ $G L(V)$, and using [SW13, Theorem B]) a $p$-divisible group $\mathcal{G} / \mathcal{O}_{C}$ with trivialized Tate module, which by functoriality comes equipped with an action of $\mathcal{O}_{B}$ and a principal polarization. To give a point of $\mathcal{M}_{\mathcal{D}, \infty}\left(C, \mathcal{O}_{C}\right)$, it remains to construct a quasi-isogeny $\rho$ over $\mathcal{O}_{C} / p$. For this, note that the proof of Proposition 4.2.6 gives an identification between the $G$-bundle $\mathcal{E}_{\mathcal{G}}$ corresponding to $\mathcal{G}$, and the $G$-bundle $\mathcal{E}_{x}$ corresponding to the point $x$. By assumption, $x \in \mathscr{F} \ell_{G, \mu}^{b}\left(C, \mathcal{O}_{C}\right)$, so there is an isomorphism of $G$-bundles $\mathcal{E}_{x} \cong \mathcal{E}_{b}$, which gives an isomorphism of $G$-bundles $\mathcal{E}_{\mathcal{G}} \cong \mathcal{E}_{b}$. Using Theorem 4.1.4 this gives the desired quasi-isogeny.

Using these results, we can compute the dimension of the strata $\mathscr{F} \ell_{G, \mu}^{b} \subset \mathscr{F} \ell_{G, \mu}$. Here, we define the dimension as the Krull dimension, i.e. the length of the longest chain of specializations.

Proposition 4.2.19. Let $K$ be a complete nonarchimedean field with ring of integers $\mathcal{O}_{K}$ and residue field $k$. Let $X$ be a partially proper adic space over $\operatorname{Spa}\left(K, \mathcal{O}_{K}\right)$. Then the dimension of $X$ is equal to the maximal transcendence degree of $k(x)$ for $x \in X$, where $k(x)$ is the residue field of the ring of integers $\mathcal{O}_{K(x)}$ in the completed residue field $K(x)$ at $x$.

Remark 4.2.20. Recall that a map $f: X \rightarrow Y$ of analytic adic spaces is partially proper if for any complete nonarchimedean field $K$ with ring of integers $\mathcal{O}_{K} \subset K$ and open and bounded valuation subring $K^{+} \subset K$ (so $K^{+} \subset \mathcal{O}_{K}$ ), the map

$$
X\left(K, K^{+}\right) \rightarrow X\left(K, \mathcal{O}_{K}\right) \times_{Y\left(K, \mathcal{O}_{K}\right)} Y\left(K, K^{+}\right)
$$

is a bijection. This is the analogue of the valuative criterion for properness in this setup. 
Proof. As $X$ lives over $\operatorname{Spa}\left(K, \mathcal{O}_{K}\right)$, it is analytic, and thus any point generalizes to a rank 1 point. It is thus enough to prove the more precise assertion that for any rank 1 point $x$, the dimension of the closure $\overline{\{x\}}$ is equal to the transcendence degree of $k(x)$. But the closure $\overline{\{x\}}$ gets identified with the Zariski-Riemann space for $k(x) / k$ (using partial properness), whose dimension is equal to the transcendence degree of $k(x) / k$.

Proposition 4.2.21. Let $K$ be a complete nonarchimedean field with ring of integers $\mathcal{O}_{K}$ and residue field $k$. Let $f: X \rightarrow Y$ be map of partially proper adic spaces over $\operatorname{Spa}\left(K, \mathcal{O}_{K}\right)$, and fix a rank 1 point $x \in X$, with image $y \in Y$. Let $X_{y}=X \times_{Y}\{y\}$ be the fibre of $f$ over $y$. Let $\overline{\{x\}}^{X} \subset X, \overline{\{y\}}^{Y} \subset Y$ and $\overline{\{x\}}^{X_{y}} \subset X_{y}$ be the respective closure. Then

$$
\operatorname{dim} \overline{\{x\}}^{X}=\operatorname{dim} \overline{\{y\}}^{Y}+\operatorname{dim} \overline{\{x\}}^{X_{y}} .
$$

Proof. Let $k(x)$ and $k(y)$ have the same meaning as in Proposition4.2.19, Then the statement translates into the additivity of transcendence degrees for the extensions $k(x) / k(y) / k$.

Proposition 4.2.22. For any complete nonarchimedean field $K / \mathcal{O}_{\breve{E}}$, the space

$$
\operatorname{Aut}_{G}\left(\widetilde{\mathbb{X}}_{b}\right)^{\text {ad }} \times_{\operatorname{Spa}\left(\mathcal{O}_{\breve{E}}, \mathcal{O}_{\breve{E}}\right)} \operatorname{Spa}\left(K, \mathcal{O}_{K}\right)
$$

is partially proper over $\operatorname{Spa}\left(K, \mathcal{O}_{K}\right)$, of dimension $\left\langle 2 \rho, \nu_{b}\right\rangle$.

Proof. The adic generic fiber is partially proper by Lemma 4.2.15. (A quasi-selfisogeny respecting extra structures over $\mathrm{Spa}\left(C, \mathcal{O}_{C}\right)$ will also respect the extra structures when it extends to $\mathrm{Spa}\left(C, C^{+}\right)$by the injectivity of the map in Lemma 4.2.16, ) For the claim about the dimension of $\operatorname{Aut}_{G}\left(\widetilde{\mathbb{X}}_{b}\right)^{\text {ad }} \times_{\mathrm{Spa}\left(\mathcal{O}_{\breve{E}}, \mathcal{O}_{\breve{E}}\right)} \operatorname{Spa}\left(K, \mathcal{O}_{K}\right)$, it is enough to consider a connected component, all of which are by Proposition 4.2 .11 given by

$$
\operatorname{Spa}\left(\mathcal{O}_{\breve{E}}\left[\left[x_{1}^{1 / p^{\infty}}, \ldots, x_{d}^{1 / p^{\infty}}\right]\right], \mathcal{O}_{\breve{E}}\left[\left[x_{1}^{1 / p^{\infty}}, \ldots, x_{d}^{1 / p^{\infty}}\right]\right]\right) \times_{\operatorname{Spa}\left(\mathcal{O}_{\breve{E}}, \mathcal{O}_{\breve{E}}\right)} \operatorname{Spa}\left(K, \mathcal{O}_{K}\right) .
$$

To compute the dimension, we may assume that $K$ is algebraically closed. Then $K$ is perfectoid, and by tilting we can assume that $K$ is of characteristic $p$. In that case, the space is topologically the same as

$$
\operatorname{Spa}\left(\mathcal{O}_{\breve{E}}\left[\left[x_{1}, \ldots, x_{d}\right]\right], \mathcal{O}_{\breve{E}}\left[\left[x_{1}, \ldots, x_{d}\right]\right]\right) \times_{\operatorname{Spa}\left(\mathcal{O}_{\breve{E}}, \mathcal{O}_{\breve{E}}\right)} \operatorname{Spa}\left(K, \mathcal{O}_{K}\right) .
$$

But this is the $d$-dimensional open unit disc over $K$.

Proposition 4.2.23. The dimension of $\mathscr{F}_{G, \mu}^{b}$ is equal to $\langle 2 \rho, \mu\rangle-\left\langle 2 \rho, \nu_{b}\right\rangle$.

Proof. Both $\mathscr{F} \ell_{G, \mu}$ and $\mathcal{M}_{\mathcal{D}, \infty}$ are partially proper adic spaces over $\operatorname{Spa}\left(\breve{E}, \mathcal{O}_{\breve{E}}\right)$ of dimension $\langle 2 \rho, \mu\rangle$. Pick any rank 1 point $x \in \mathcal{M}_{\mathcal{D}, \infty}$ such that the dimension of $\overline{\{x\}}$ is $\langle 2 \rho, \mu\rangle$, and let $y \in \mathscr{F}_{G, \mu}^{b}$ be its image. Let $\bar{y}$ be a geometric point above $y$, corresponding to a completed algebraic closure $C$ of $K(y)$, and pick a lift of $\bar{y}$ to $\mathcal{M}_{\mathcal{D}, \infty}$, using Lemma 4.2.18. Then Proposition 4.2.21 shows that

$$
\langle 2 \rho, \mu\rangle \leq \operatorname{dim} \overline{\{y\}}+\operatorname{dim} \mathcal{M}_{\mathcal{D}, \infty, y} .
$$

But $\operatorname{dim} \mathcal{M}_{\mathcal{D}, \infty, y}=\operatorname{dim} \mathcal{M}_{\mathcal{D}, \infty, \bar{y}}$, and using Proposition 4.2.14 and the choice of $\bar{y}$, one has

$$
\operatorname{dim} \mathcal{M}_{\mathcal{D}, \infty, \bar{y}}=\operatorname{dim} \operatorname{Aut}_{G}\left(\widetilde{\mathbb{X}}_{b}\right)^{\text {ad }} \times_{\operatorname{Spa}\left(\mathcal{O}_{\breve{E}}, \mathcal{O}_{\breve{E}}\right)} \operatorname{Spa}\left(C, \mathcal{O}_{C}\right) .
$$


The latter has been computed in Proposition 4.2.22, showing the inequality

$$
\operatorname{dim} \mathscr{F}_{G, \mu}^{b} \geq \operatorname{dim} \overline{\{y\}} \geq\langle 2 \rho, \mu\rangle-\left\langle 2 \rho, \nu_{b}\right\rangle .
$$

For the converse, pick any rank 1 point $y \in \mathscr{F} \ell_{G, \mu}$. As before, one sees that $\operatorname{dim} \mathcal{M}_{\mathcal{D}, \infty, y}=\left\langle 2 \rho, \nu_{b}\right\rangle$, so pick a rank 1 point $x \in \mathcal{M}_{\mathcal{D}, \infty, y}$ whose closure is of dimension $\left\langle 2 \rho, \nu_{b}\right\rangle$. Applying Proposition 4.2.21, we see that the dimension of the closure of $x$ in $\mathcal{M}_{\mathcal{D}, \infty}$ is at least $\operatorname{dim} \overline{\{y\}}+\left\langle 2 \rho, \nu_{b}\right\rangle$. On the other hand, the dimension of the closure of $x$ is bounded by $\operatorname{dim} \mathcal{M}_{\mathcal{D}, \infty}=\langle 2 \rho, \mu\rangle$. This shows that

$$
\operatorname{dim} \overline{\{y\}} \leq\langle 2 \rho, \mu\rangle-\left\langle 2 \rho, \nu_{b}\right\rangle,
$$

which (as $y$ was arbitrary) proves the other inequality.

4.3. A product formula. We now return to our global setting, where we want to study the Hodge-Tate period map $\pi_{H T}: \mathcal{S}_{K^{p}} \rightarrow \mathscr{F}_{G}$. Recall that we are restricting to the case when the Shimura datum $(G, X)$ is of PEL type.

More precisely, we fix global PEL data as follows, cf. [Kot92b, §5]. Let $B$ be a finite-dimensional simple $\mathbb{Q}$-algebra with center $F$, and let $V$ be a faithful finitely generated $B$-representation. Let $*$ be a positive involution on $B$, and $F^{+}=F^{*=1}$. On $V$, we fix a nondegenerate $\mathbb{Q}$-valued alternating form $(\cdot, \cdot)$ such that $(b v, w)=$ $\left(v, b^{*} w\right)$ for all $v, w \in V$ and $b \in B$. Let $G / \mathbb{Q}$ be the algebraic group whose $R$-valued points are

$$
G(R)=\left\{x \in \operatorname{End}_{B \otimes R}(V \otimes R) \mid x x^{*} \in R^{\times}\right\} .
$$

We assume that $G$ is connected; under the classification of Kot92b], this amounts to excluding type D. Finally, we fix a $*$-homomorphism $h: \mathbb{C} \rightarrow \operatorname{End}_{B \otimes \mathbb{R}}(V \otimes \mathbb{R})$ such that the symmetric real-valued bilinear form $(v, h(i) w)$ on $V \otimes \mathbb{R}$ is positivedefinite. Note that $h$ induces a map, denoted in the same way, $h: \operatorname{Res}_{\mathbb{C} / \mathbb{R}} \rightarrow G_{\mathbb{R}}$, and in particular a Shimura datum.

We need to assume that these data are "unramified" at $p$. More precisely, we assume that $B_{\mathbb{Q}_{p}}$ is a product of matrix algebras over unramified extensions of $\mathbb{Q}_{p}$,

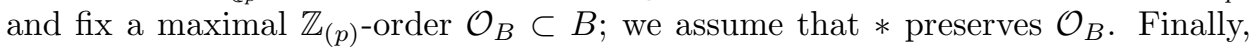
we assume that there exists a $\mathbb{Z}_{(p)}$-lattice $\Lambda \subset V$ that is self-dual under $(\cdot, \cdot)$ and stable under $\mathcal{O}_{B}$, and we fix such a $\Lambda$. Using these data, we can define a connected reductive group $G_{\mathbb{Z}_{(p)}}$ over $\mathbb{Z}_{(p)}$ with generic fibre $G$ as

$$
G_{\mathbb{Z}_{(p)}}(R)=\left\{x \in \operatorname{End}_{\mathcal{O}_{B} \otimes R}(\Lambda \otimes R) \mid x x^{*} \in R^{\times}\right\} .
$$

We fix the hyperspecial maximal compact open subgroup $K_{p}=G_{\mathbb{Z}_{(p)}}\left(\mathbb{Z}_{p}\right) \subset G\left(\mathbb{Q}_{p}\right)$.

Let $K^{p} \subset G\left(\mathbb{A}_{f}^{p}\right)$ be a compact open subgroup, and fix a place $\mathfrak{p} \mid p$ of $E$. As in Kot92b], one can define a moduli space of abelian varieties with extra structures $\mathscr{S}_{K_{p} K^{p}}$ over $\mathcal{O}_{E, \mathfrak{p}} \subset E$. In most cases, the generic fibre $S_{K_{p} K^{p}} / E$ of $\mathscr{S}_{K_{p} K^{p}}$ is the Shimura variety corresponding to $(G,\{h\})$; in general, however, the Hasse principle for the group $G$ fails, and it consists of $\left|\operatorname{ker}^{1}(\mathbb{Q}, G)\right|$ copies of this Shimura variety. Thus, the notation of this section conflicts slightly with the previous notation for Shimura varieties of Hodge type.

Let $\mathbb{F}_{q}$ be the residue field of $\mathcal{O}_{E, \mathfrak{p}}$. The special fiber $\mathscr{S}_{K_{p} K^{p}} \times_{\mathcal{O}_{E, \mathfrak{p}}} \mathbb{F}_{q}$ admits a Newton stratification by locally closed strata $\mathscr{S}_{K_{p} K^{p}}^{b}$ indexed by $b \in B\left(G, \mu^{-1}\right)$, cf. [RR96: A point $x \in \mathscr{S}_{K_{p} K^{p}} \times_{\mathcal{O}_{E, \mathfrak{p}}} \mathbb{F}_{q}$ gives rise to a $p$-divisible group with extra structure, which can be translated into an isocrystal with $G$-structure, and is classified by an element $b \in B(G)$. By [RR96, this element actually lies in $B\left(G, \mu^{-1}\right)$. 
One of the main results of Man05] is a decomposition of the Newton stratum $\mathscr{S}_{K_{p} K^{p}}^{b}$ into the Rapoport-Zink space $\mathfrak{M}^{b}$ and the Igusa variety $\operatorname{Ig}^{b}$ corresponding to $b$. Thus, we first recall these two objects. From the last section, we already know the Rapoport-Zink space:

For $b \in B\left(G, \mu^{-1}\right)$, choose a completely slope divisible $p$-divisible group $\mathbb{X}_{b}$ over $\overline{\mathbb{F}}_{q}$ with extra structures giving rise to the $\sigma$-conjugacy class $b$, as in Man05, §3]. Let $\mathcal{D}_{\text {int }, b}$ be the integral data corresponding to the base extension of $B, V, \mathcal{O}_{B}, \Lambda$ to $\mathbb{Z}_{p}$, and $(\mu, b)$. Then $\mathcal{D}_{\text {int }, b}$ is of PEL type, and we consider the corresponding Rapoport-Zink space $\mathfrak{M}^{b}:=\mathfrak{M}_{\mathcal{D}_{\text {int }, b}}$, which lives over $\mathcal{O}_{\breve{E}}$, where $\breve{E}$ is the completion of the maximal unramified extension of $E_{\mathfrak{p}}$.

Next, we want to introduce the Igusa variety.

Definition 4.3.1. We let $\operatorname{Ig}^{b} /$ Spec $\overline{\mathbb{F}}_{q}$ be the functor sending an $\overline{\mathbb{F}}_{q}$-algebra $R$ to the set of isomorphism classes of pairs

$$
\left\{(A, \rho) \mid A \in \mathscr{S}_{K_{p} K^{p}}(R), \rho: A\left[p^{\infty}\right] \stackrel{\sim}{\rightarrow} \mathbb{X}_{b} \times_{\overline{\mathbb{F}}_{p}} R\right\},
$$

where $A \in \mathscr{S}_{K_{p} K^{p}}(R)$ is an abelian variety equipped with extra structures (and satisfying the determinant condition) and the isomorphism $\rho$ is compatible with the extra structures; as usual, it is only supposed to preserve the polarization up to a scalar, i.e. an automorphism of $\mu_{p \infty, R}$.

Remark 4.3.2. This definition is different from the Igusa varieties defined in [Man05], and we will explain their relation below.

Proposition 4.3.3. The functor $\mathrm{Ig}^{b}$ is representable by a scheme.

Proof. It is enough to prove that the map $\operatorname{Ig}^{b} \rightarrow \mathscr{S}_{K_{p} K^{p}} \times_{\mathcal{O}_{E, p}} \overline{\mathbb{F}}_{q}$ is relatively representable. Let $\mathcal{A}$ be the universal abelian variety over $\mathscr{S}_{K_{p} K^{p}}$. Then we are considering the inverse limit of the schemes parametrizing isomorphisms $\mathcal{A}\left[p^{n}\right] \cong$ $\mathbb{X}_{b}\left[p^{n}\right]$ compatible with extra structures, each of which is representable.

From the definition of $\operatorname{Ig}^{b}$, it is evident that the group of automorphisms of $\mathbb{X}_{b}$ respecting the extra structures acts on it. However, we give next an alternative description of $\operatorname{Ig}^{b}$ which shows that the larger group $\operatorname{Aut}_{G}\left(\widetilde{\mathbb{X}}_{b}\right)$ acts on $\operatorname{Ig}^{b}$.

Lemma 4.3.4. For an $\overline{\mathbb{F}}_{q}$-algebra $R, \operatorname{Ig}^{b}(R)$ can be identified with the set of isomorphism classes of pairs $(A, \tilde{\rho})$, where $A \in \mathscr{S}_{K_{p} K^{p}}(R)$ is an abelian variety considered up to $p$-power isogeny (respecting the extra structures) and

$$
\rho: A\left[p^{\infty}\right] \stackrel{\sim}{\rightarrow} \mathbb{X}_{b} \times_{\overline{\mathbb{F}}_{p}} R
$$

is a quasi-isogeny (respecting the extra structures).

Proof. Each element $(A, \rho)$ of $\operatorname{Ig}^{b}(R)$ determines a pair $(A, \rho)$ as in the statement of the lemma.

Conversely, given $A \in \mathscr{S}_{K_{p} K^{p}}(R)$ with a quasi-isogeny

$$
\rho: A\left[p^{\infty}\right] \stackrel{\sim}{\rightarrow} \mathbb{X}_{b} \times_{\overline{\mathbb{F}}_{p}} R,
$$

we can find a unique abelian variety $A^{\prime}$ with extra structures equipped with a $p$-power isogeny to $A$, such that $A\left[p^{\infty}\right]$ gets identified with $\mathbb{X}_{b}$, i.e. the induced quasi-isogeny

$$
\rho^{\prime}: A^{\prime}\left[p^{\infty}\right] \stackrel{\sim}{\rightarrow} \mathbb{X}_{b} \times_{\overline{\mathbb{F}}_{p}} R
$$

is an isomorphism. Then $\left(A^{\prime}, \rho^{\prime}\right)$ defines a point of $\operatorname{Ig}^{b}(R)$, as desired. 
Corollary 4.3.5. The formal group scheme $\operatorname{Aut}_{G}\left(\widetilde{\mathbb{X}}_{b}\right)$ acts canonically on $\operatorname{Ig}^{b}$. Moreover, $\mathrm{Ig}^{b}$ is perfect, i.e. the Frobenius map is an automorphism.

Proof. The first part follows from Lemma 4.3.4 by acting on $\rho$ (noting that quasiisogenies of $\mathbb{X}_{b}$ are the same as automorphisms of $\widetilde{\mathbb{X}}_{b}$ ).

For the second part, we have to see that for any $\overline{\mathbb{F}}_{q}$-algebra $R$, the Frobenius

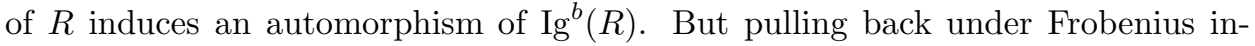
duces an equivalence on the category of abelian varieties up to $p$-power isogeny (as Verschiebung gives an inverse up to multiplication by $p$ ). Similarly, pull back under Frobenius induces an equivalence on the category of $p$-divisible groups up to quasi-isogeny, showing that the datum of $\rho$ is preserved.

Now we recall the more classical objects; for more details, see [Man05]. The leaf $\mathscr{C}^{b}$ corresponding to $\mathbb{X}_{b}$ is the subset of the locally closed stratum $\mathscr{S}_{K_{p} K^{p}}^{b} \times_{\mathbb{F}_{q}} \overline{\mathbb{F}}_{q}$ where the fibers of the $p$-divisible group $\mathcal{A}\left[p^{\infty}\right]$ at all geometric points are isomorphic to $\mathbb{X}_{b}$ :

$$
\mathscr{C}^{b}:=\left\{x \in \mathscr{S}_{K}^{b} \mid \mathcal{A}_{x}\left[p^{\infty}\right] \times_{\kappa(x)} \overline{\kappa(x)} \simeq \mathbb{X}_{b} \times_{\overline{\mathbb{F}}_{p}} \overline{\kappa(x)}\right\} .
$$

This is a priori defined only as a subset of $\mathscr{S}_{K_{p} K^{p}}^{b} \times_{\mathbb{F}_{q}} \overline{\mathbb{F}}_{q}$, but Proposition 1 of Man05] shows that $\mathscr{C}^{b}$ is a closed subset and defines a smooth subscheme of $\mathscr{S}_{K_{p} K^{p}}^{b} \times_{\mathbb{F}_{q}} \overline{\mathbb{F}}_{q}$ when endowed with the induced reduced structure. We note that contrary to the objects defined so far, $\mathscr{C}^{b}$ depends on the choice of $\mathbb{X}_{b}$ within its isogeny class.

Recall that

$$
\mathbb{X}_{b}=\oplus_{i=1}^{r} \mathbb{X}_{i}
$$

where the $\mathbb{X}_{i}$ are isoclinic $p$-divisible groups of strictly decreasing slopes $\lambda_{i} \in[0,1]$. Let $\mathcal{G}_{b}$ be the $p$-divisible group of the universal abelian variety $\mathscr{A} / \mathscr{S}_{K_{p} K^{p}}$ restricted to $\mathscr{C}^{b}$. Then $\mathcal{G}_{b}$ is completely slope divisible, with slope filtration

$$
0 \subset \mathcal{G}_{b, 1} \subset \cdots \subset \mathcal{G}_{b, r}=\mathcal{G}_{b},
$$

with $\mathcal{G}_{b}^{i}:=\mathcal{G}_{b, i} / \mathcal{G}_{b, i-1}$ isoclinic of slope $\lambda_{i}$. The $\mathcal{O}_{B}$-action on $\mathcal{G}$ and the polarization respect this filtration, so that each $\mathcal{G}_{b}^{i}$ is endowed with an $\mathcal{O}_{B}$-action and there are induced polarizations $\mathcal{G}_{b}^{i} \rightarrow\left(\mathcal{G}_{b}^{j}\right)^{\vee}$ for all $i, j$ with $\lambda_{i}+\lambda_{j}=1$.

Definition 4.3.6. The (pro-)Igusa variety is the map

$$
\mathscr{I}_{\text {Mant }}^{b} \rightarrow \mathscr{C}^{b}
$$

which over a $\mathscr{C}^{b}$-scheme $S$ parametrizes tuples $\left(\rho_{i}\right)_{i=1}^{r}$ of isomorphisms

$$
\rho_{i}: \mathcal{G}_{b}^{i} \times_{\mathscr{C} b} S \stackrel{\sim}{\rightarrow} \mathbb{X}_{i} \times_{\text {Spec }} \overline{\mathbb{F}}_{p} S
$$

which are compatible with the $\mathcal{O}_{B}$-actions on $\mathcal{G}_{b}^{i}$ and $\mathbb{X}_{i}$, and commute with the polarizations on $\mathcal{G}$ and $\mathbb{X}_{b}$, up to an automorphism of $\mu_{p \infty}, S$.

Remark 4.3.7. A version of these Igusa varieties is considered in [Man05] (see also Section II of [HT01 for the case of one-dimensional $p$-divisible groups). Rather than trivializing the whole isoclinic $p$-divisible group $\mathcal{G}_{b}^{i}$, one trivializes the $\mathcal{G}_{b}^{i}\left[p^{m}\right]$ for some positive integer $m$. More precisely, let $\mathscr{I}_{\text {Mant,m }}^{b}$ be the moduli space of isomorphisms on $\mathscr{C}^{b}$-schemes $S$

$$
\rho_{i, m}: \mathcal{G}_{b}^{i}\left[p^{m}\right] \stackrel{\sim}{\rightarrow} \mathbb{X}_{i}\left[p^{m}\right] \times_{\overline{\mathbb{F}}_{p}} S,
$$


which (fppf locally) lift to arbitrary $m^{\prime} \geq m$ and which respect the extra structures. Proposition 4 of [Man05] shows that the underlying reduced subscheme of $\mathscr{I}_{\text {Mant, } m}^{b}$ is a finite étale and Galois cover of $\mathscr{C}^{b}$.

In view of the theory developed in Section 4.1, we can identify the set of endomorphisms of $\mathbb{X}_{i}\left[p^{m}\right]$, which lift to arbitrary $m^{\prime} \geq m$, with the $p^{m}$-torsion in the étale $p$-divisible group $\mathcal{H}_{\mathbb{X}_{i}, \mathbb{X}_{i}}$. Now consider the intersection of the scheme-theoretic images of the automorphisms of $\mathbb{X}_{i}\left[p^{m+k}\right]$ inside the automorphisms of $\mathbb{X}_{i}\left[p^{m}\right]$ (under the natural restriction map). By Lemma 4.1.5 the images of $\mathscr{A} u t\left(\mathbb{X}_{i}\left[p^{m+k}\right]\right) \hookrightarrow$ $\mathscr{A} u t\left(\mathbb{X}_{i}\left[p^{m}\right]\right)$ will stabilize for large enough $k$, giving rise to an open and closed subscheme of the finite étale scheme $\mathcal{H}_{\mathbb{X}_{i}, \mathbb{X}_{i}}\left[p^{m}\right]$. This shows that $\mathscr{I}_{\text {Mant }, m}^{b} \rightarrow \mathscr{C}^{b}$ is a quasitorsor under an étale group scheme. From [Man05, Proposition 4] (which produces sections over a finite étale cover), it follows that they are actually torsors. In particular, we see that $\mathscr{I}_{\text {Mant, } m}^{b}$ is actually already reduced.

Thus, the scheme

$$
\mathscr{I}_{\text {Mant }}^{b}={\underset{m}{\lim }}_{\mathscr{I}_{\text {Mant }, m}^{b}}
$$

is a pro-étale cover of $\mathscr{C}^{b}$.

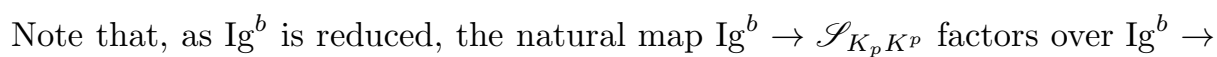
$\mathscr{C}^{b}$. Moreover, as any homomorphism between $p$-divisible groups preserves the slope filtration by Corollary 4.1.10, we see that any isomorphism $\mathcal{G}_{b} \cong \mathbb{X}_{b}$ induces isomorphisms $\mathcal{G}_{b}^{i} \cong \mathbb{X}_{i}$, and thus there is a natural map $\operatorname{Ig}^{b} \rightarrow \mathscr{I}_{\text {Mant }}^{b}$.

Proposition 4.3.8. The perfect scheme $\operatorname{Ig}^{b}$ is the perfection of $\mathscr{I}_{\text {Mant }}^{b}$, via the natural map $\operatorname{Ig}^{b} \rightarrow \mathscr{I}_{\text {Mant }}^{b}$.

Proof. Let $\left(\mathscr{I}_{\text {Mant }}^{b}\right)^{\text {perf }}$ be the perfection of $\mathscr{I}_{\text {Mant }}^{b}$. Then we claim that the $p$ divisible group $\mathcal{G}_{b}$ over $\mathscr{C}^{b}$ becomes canonically isomorphic to $\mathbb{X}_{b}$ when pulled back to $\left(\mathscr{I}_{\text {Mant }}^{b}\right)^{\text {perf }}$. Recall that $\mathcal{G}_{b}$ has a slope filtration

$$
0 \subset \mathcal{G}_{b, 1} \subset \cdots \subset \mathcal{G}_{b, r}=\mathcal{G}_{b},
$$

with $\mathcal{G}_{b}^{i}:=\mathcal{G}_{b, i} / \mathcal{G}_{b, i-1}$ isoclinic of slope $\lambda_{i}$. Moreover, when pulled back along $\mathscr{I}_{\text {Mant }}^{b} \rightarrow \mathscr{C}^{b}$, each $\mathcal{G}_{b}^{i}$ becomes trivialized to $\mathbb{X}_{i}$.

The existence of the slope filtration on $\mathcal{G}_{b}$ means that we have integers $0 \leq t_{r}<$ $\cdots<t_{2}<t_{1} \leq s$, such that for $i=1, \ldots, r$ :

(1) the slope $\lambda_{i}=\frac{t_{i}}{s}$;

(2) the quasi-isogenies

$$
\frac{F^{s}}{p^{t_{i}}}: \mathcal{G}_{b, i} \rightarrow\left(\mathcal{G}_{b, i}\right)^{\left(p^{s}\right)}
$$

where $F$ is the Frobenius isogeny, are genuine isogenies.

(3) the induced maps

are isomorphisms.

$$
\frac{F^{s}}{p^{t_{i}}}: \mathcal{G}_{b}^{i} \rightarrow\left(\mathcal{G}_{b}^{i}\right)^{\left(p^{s}\right)}
$$

The inequalities between the $t_{i}$ imply that $\frac{F^{s}}{p^{t_{i}}}$ acts nilpotently on $\mathcal{G}_{b, i-1}$. Repeated iterations of

$$
\frac{F^{s}}{p^{t_{i}}}:\left(\mathcal{G}_{b, i}\right)^{\left(p^{-s}\right)} \rightarrow \mathcal{G}_{b, i}
$$

can be used to construct canonical splittings $\mathcal{G}_{b}^{i} \hookrightarrow \mathcal{G}_{b, i}$ over $\left(\mathscr{I}_{\text {Mant }}^{b}\right)^{\text {perf }}$. 
Thus, $\mathcal{G}$ decomposes canonically into $\mathcal{G}_{1} \times \cdots \times \mathcal{G}_{r}$ over $\left(\mathscr{I}_{\text {Mant }}^{b}\right)^{\text {perf }}$, and this is trivialized to $\mathbb{X}_{1} \times \cdots \times \mathbb{X}_{r}=\mathbb{X}_{b}$, as desired.

We remark that $J_{b}\left(\mathbb{Q}_{p}\right) \subset \operatorname{Aut}_{G}\left(\widetilde{\mathbb{X}}_{b}\right)$ acts on $\operatorname{Ig}^{b}$. However, only a certain submonoid of $J_{b}\left(\mathbb{Q}_{p}\right)$ acts on $\mathscr{I}_{\text {Mant }}^{b}$; Mantovan, Man05, does however construct a canonical action of $J_{b}\left(\mathbb{Q}_{p}\right)$ on the étale cohomology of $\mathscr{I}_{\text {Mant }}^{b}$. From Proposition 4.3.8, it follows that the étale cohomology of $\mathscr{I}_{\text {Mant }}^{b}$ is also the étale cohomology of $\operatorname{Ig}^{b}$, on which we have a natural action of $J_{b}\left(\mathbb{Q}_{p}\right)$. We leave it to the reader to verify that this is the same action as the one constructed by Mantovan.

Corollary 4.3.9. The map $\operatorname{Ig}^{b} \rightarrow \mathscr{C}^{b}$ is faithfully flat.

As the map is obviously a quasitorsor under the automorphisms of $\mathbb{X}_{b}$ respecting the extra structure, this implies that it is in fact a torsor under this group. Note that $\mathscr{C}^{b}$ is smooth, while the scheme of automorphisms of $\mathbb{X}_{b}$ is a highly nonreduced object like Spec$\overline{\mathbb{F}}_{p}\left[\left[X_{1}^{1 / p^{\infty}}, \ldots, X_{d}^{1 / p^{\infty}}\right]\right] /\left(X_{1}, \ldots, X_{d}\right)$. The fact that a torsor under this group over something smooth is a perfect scheme forces the smooth directions of the base to match with the nonreduced directions of the group, so that one can deduce that the dimension of $\mathscr{C}^{b}$ is $d=\left\langle 2 \rho, \nu_{b}\right\rangle$, for example by looking at the transitivity triangle for the cotangent complex.

Proof. As $\mathscr{I}_{\text {Mant }}^{b}$ is a cofiltered limit of smooth schemes along affine transition maps, its Frobenius morphism is (faithfully) flat, and thus $\operatorname{Ig}^{b} \rightarrow \mathscr{I}_{\text {Mant }}^{b}$ is faithfully flat. We have already seen that $\mathscr{I}_{\text {Mant }}^{b} \rightarrow \mathscr{C}^{b}$ is faithfully flat, so we get the result.

$\mathrm{As} \mathrm{g}^{b}$ is a perfect scheme, it lifts uniquely to a flat $p$-adic formal scheme over $W\left(\overline{\mathbb{F}}_{q}\right)=\mathcal{O}_{\breve{E}}$, which we denote by $\operatorname{Ig}_{\mathcal{O}_{\breve{E}}^{b}}^{b}$. As a moduli problem on $\mathrm{Nilp}_{\mathcal{O}_{\breve{E}}}^{\text {op }}$, it parametrizes abelian varieties up to $p$-power isogeny in $\mathscr{S}_{K_{p} K^{p}}$, equipped with an isomorphism of $\widetilde{A\left[p^{\infty}\right]}$ with (the canonical lift of) $\widetilde{\mathbb{X}}_{b}$, respecting all extra structures.

One can also describe this deformation of $\operatorname{Ig}^{b}$ to mixed characteristic differently. For this, fix a lift $\left(\mathbb{X}_{b}\right)_{\mathcal{O}_{K}}$ of $\mathbb{X}_{b}$ up to quasi-isogeny (with its extra structures) to $\mathcal{O}_{K}$, where $\mathcal{O}_{K}$ is the ring of integers of some complete nonarchimedean field $K / \breve{E}$; in other words, pick a point $\left(\mathbb{X}_{b}\right)_{\mathcal{O}_{K}} \in \mathfrak{M}^{b}\left(\mathcal{O}_{K}\right)$. This is possible (with $K=\breve{E}$ ), as $\mathfrak{M}^{b}$ is formally smooth. One gets the following lemma.

Lemma 4.3.10. The points of the formal scheme $\operatorname{Ig}_{\mathcal{O}_{K}}^{b}=\operatorname{Ig}_{\mathcal{O}_{\breve{E}}}^{b} \times_{\mathcal{O}_{\breve{E}}} \mathcal{O}_{K}$ over $R \in \operatorname{Nilp}_{\mathcal{O}_{K}}^{\text {op }}$ are given by the pairs $(A, \rho)$, where $A \in \mathscr{S}_{K_{p} K^{p}}(R)$ is an abelian variety with extra structure, and

$$
\rho: A\left[p^{\infty}\right] \stackrel{\sim}{\rightarrow}\left(\mathbb{X}_{b}\right)_{\mathcal{O}_{K}} \times \mathcal{O}_{K} R
$$

is an isomorphism compatible with the extra structure.

We will also need a variant of Igusa varieties, where one trivializes $A\left[p^{\infty}\right]$ only up to quasi-isogeny.

Definition 4.3.11. Let $\mathfrak{X}^{b}$ be the functor sending $R \in \operatorname{Nilp}_{\mathcal{O}_{E, \mathfrak{p}}}^{\text {op }}$ to the set of pairs $(A, \rho)$, where $A \in \mathscr{S}_{K_{p} K^{p}}(R)$ is an abelian variety with extra structure, and

$$
\rho: A\left[p^{\infty}\right] \times_{R} R / p \rightarrow \mathbb{X}_{b} \times_{\overline{\mathbb{F}}_{q}} R / p
$$

is a quasi-isogeny compatible with the $\mathcal{O}_{B}$-action and the polarization, up to an automorphism of $\widetilde{\mu}_{p^{\infty}, R / p}$. 
Fix a lift $\left(\mathbb{X}_{b}\right)_{\mathcal{O}_{K}}$ of $\mathbb{X}_{b}$ to $\mathcal{O}_{K}$ as above. We define a map of formal schemes over $\mathcal{O}_{K}$,

For $R \in \operatorname{Nilp}_{\mathcal{O}_{K}}^{\text {op }}$, let

$$
\operatorname{Ig}_{\mathcal{O}_{K}}^{b} \times_{\mathcal{O}_{\check{E}}} \mathfrak{M}^{b} \rightarrow \mathfrak{X}_{\mathcal{O}_{K}}^{b}
$$

$$
(A, \rho),\left(\mathcal{G}, \rho^{\prime}\right) \in\left(\operatorname{Ig}_{\mathcal{O}_{K}}^{b} \times \mathfrak{M}^{b}\right)(R) .
$$

Thus, $A \in \mathscr{S}_{K_{p} K^{p}}(R)$ is an abelian variety with extra structure, equipped with an isomorphism

$$
\rho: A\left[p^{\infty}\right] \cong\left(\mathbb{X}_{b}\right)_{\mathcal{O}_{K}} \times_{\mathcal{O}_{K}} R
$$

On the other hand, $\mathcal{G}$ is a $p$-divisible group with extra structure over $R$, equipped with a quasi-isogeny $\rho^{\prime}$ to $\mathbb{X}_{b}$ over $R / p$, which lifts uniquely to a quasi-isogeny (denoted in the same way)

$$
\rho^{\prime}: \mathcal{G} \rightarrow\left(\mathbb{X}_{b}\right)_{\mathcal{O}_{K}} \times \mathcal{O}_{K} R .
$$

We get the composite quasi-isogeny $\mathcal{G} \rightarrow A\left[p^{\infty}\right]$. It follows that there is a unique quasi-isogeny of $p$-power order $A^{\prime} \rightarrow A$ such that $A^{\prime}\left[p^{\infty}\right] \rightarrow A\left[p^{\infty}\right]$ gets identified with $\mathcal{G} \rightarrow A\left[p^{\infty}\right]$. This defines a new point $A^{\prime} \in \mathscr{S}_{K_{p} K^{p}}(R)$, which comes equipped with a quasi-isogeny

$$
\rho^{\prime}: A^{\prime}\left[p^{\infty}\right]=\mathcal{G} \rightarrow\left(\mathbb{X}_{b}\right)_{\mathcal{O}_{K}} \times_{\mathcal{O}_{K}} R,
$$

and in particular a quasi-isogeny to $\mathbb{X}_{b}$ over $R / p$.

Lemma 4.3.12. The map constructed above induces an isomorphism, and fits into a commutative diagram

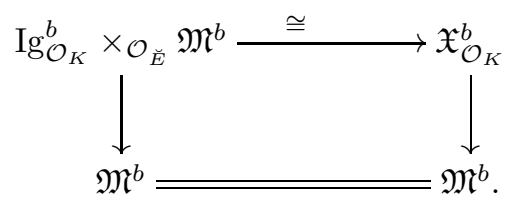

Here, the first vertical map is projection onto the second factor, and the second vertical map sends $(A, \rho) \in \mathfrak{X}^{b}$ to $\left(A\left[p^{\infty}\right], \rho\right) \in \mathfrak{M}^{b}$.

In particular, choosing $K=\breve{E}$ above, $\mathfrak{X}^{b}$ is representable by a formal scheme.

Proof. The diagram commutes by construction.

We now define the inverse of the top horizontal map: suppose we are given a pair $\left(A^{\prime}, \rho^{\prime}\right) \in \mathfrak{X}^{b}(R)$. In order to define $\left(\mathcal{G}, \rho^{\prime}\right) \in \mathfrak{M}^{b}(R)$ we just take $\left(A^{\prime}\left[p^{\infty}\right], \rho^{\prime}\right)$. From the quasi-isogeny

$$
\rho^{\prime}: A^{\prime}\left[p^{\infty}\right] \rightarrow\left(\mathbb{X}_{b}\right)_{\mathcal{O}_{K}} \times_{\mathcal{O}_{K}} R,
$$

we find a quasi-isogeny of $p$-power degree $A^{\prime} \rightarrow A$ such that the induced quasiisogeny

$$
\rho: A\left[p^{\infty}\right] \rightarrow\left(\mathbb{X}_{b}\right)_{\mathcal{O}_{K}} \times_{\mathcal{O}_{K}} R
$$

is an isomorphism, so we get $(A, \rho) \in \operatorname{Ig}_{\mathcal{O}_{K}}^{b}(R)$. It is easy to verify that this construction is inverse to the horizontal map.

We would like to say that $\mathfrak{X}^{b}$ is an $\operatorname{Aut}_{G}\left(\widetilde{\mathbb{X}}_{b}\right)$-torsor over the completion of $\mathscr{S}_{K_{p} K^{p}}$ along $\mathscr{S}_{K_{p} K^{p}}^{b}$. It is clear that it is a quasitorsor, and it remains to show that the map is locally surjective in some topology, the naive choice of course being the fpqc topology. 
If this were true, then one could take the pushout along $\operatorname{Aut}_{G}\left(\widetilde{\mathbb{X}}_{b}\right) \rightarrow J_{b}\left(\mathbb{Q}_{p}\right)$ to get a $J_{b}\left(\mathbb{Q}_{p}\right)$-torsor over $\mathscr{S}_{K_{p} K^{p}}^{b}$. This $J_{b}\left(\mathbb{Q}_{p}\right)$-torsor can in fact be constructed, as in the following proposition (which will not be used in the sequel, but is included as it fits the current discussion).

Proposition 4.3.13. Let $S$ be a scheme over $\overline{\mathbb{F}}_{p}$, and let $\mathbb{X}$ be a $p$-divisible group with extra structure over $S$. Assume that there is some $b \in B(G)$ such that all fibres of $\mathbb{X}$ are quasi-isogenous to $\mathbb{X}_{b}$ (compatibly with extra structures). Then there is a natural $J_{b}\left(\mathbb{Q}_{p}\right)$-torsor over $S$ which above any geometric point $\bar{x} \in S$ parametrizes quasi-isogenies between $\mathbb{X}_{\bar{x}}$ and $\mathbb{X}_{b}$ (compatible with extra structures).

Remark 4.3.14. The $J_{b}\left(\mathbb{Q}_{p}\right)$-torsor is to be understood as in [BS15a]; more precisely, there is a sheaf of (abstract) groups on $S_{\text {proét }}$ corresponding to the topological group $J_{b}\left(\mathbb{Q}_{p}\right)$, and we are considering a torsor on $S_{\text {proét }}$ under this sheaf of groups. If $S$ is connected and locally topologically noetherian and $\bar{x} \in S$ is a geometric base point, this corresponds to a map

$$
\pi_{1}^{\text {proét }}(S, \bar{x}) \rightarrow J_{b}\left(\mathbb{Q}_{p}\right) .
$$

This map, and the $J_{b}\left(\mathbb{Q}_{p}\right)$-torsor, only depend on $\mathbb{X}$ up to isogeny. We remark that the displayed map may have noncompact image in general, but the image is compact in case $\mathbb{X}$ admits a slope decomposition (or is isogenous to such an $\mathbb{X}$ ); this explains OZ02, Example 4.2], where a $p$-divisible group over a non-normal base is constructed which is not isogenous to one admitting a slope filtration. We remark that most Newton strata, e.g. the basic one, give such examples: For the basic Newton stratum, the image of the displayed homomorphism is a discrete cocompact subgroup of $J_{b}\left(\mathbb{Q}_{p}\right)$ related to $p$-adic uniformization.

Proof. We may assume that $S$ is perfect. In that case, we consider the functor sending any $T \in S_{\text {proét }}$ to the set of quasi-isogenies between $\mathbb{X}_{T}$ and $\left(\mathbb{X}_{b}\right)_{T}$, respecting extra structures. This is a $J_{b}\left(\mathbb{Q}_{p}\right)$-quasitorsor, and we want to prove that it is a torsor.

First, we check this when $S$ is strictly local, so assume $S=\operatorname{Spec} R$ is the spectrum of a strictly henselian perfect $\operatorname{ring} R$. In that case, we need to show that there is a quasi-isogeny between $\mathbb{X}$ and $\mathbb{X}_{b}$, compatible with extra structures. As there is such a quasi-isogeny over the special point, the result follows from the following lemma.

Lemma 4.3.15. Let $R$ be a strictly henselian perfect ring with residue field $k$. Then the functor $G \mapsto G_{k}$ from the category of p-divisible groups over $R$ with constant Newton polygon, up to isogeny, to p-divisible groups over $k$ up to isogeny is an equivalence of categories.

Remark 4.3.16. In fact, the proof will show that if $G$ and $H$ are $p$-divisible groups with constant Newton polygon over $R$, then there is a constant $c$ depending only on the heights of $G$ and $H$ such that for any homomorphism $\psi_{k}: G_{k} \rightarrow H_{k}$ over $k$, $p^{c} \psi_{k}$ lifts to a (necessarily unique) homomorphism $G \rightarrow H$. (Cf. OZ02, Corollary 3.4].)

Proof. Choose an embedding $\overline{\mathbb{F}}_{p} \hookrightarrow R$. Assume for the moment that we know that any $p$-divisible group $G$ over $R$ with constant Newton polygon is isogenous to $G_{0, R}:=G_{0} \times_{\overline{\mathbb{F}}_{p}} R$ for some $p$-divisible group $G_{0}$ over $\overline{\mathbb{F}}_{p}$. By the DieudonnéManin classification, the functor in the lemma is essentially surjective. To check 
fully faithfulness of the functor, we may restrict to calculating $\operatorname{Hom}_{R}(G, H)[1 / p]$ where $G=G_{0, R}, H=H_{0, R}$. By fully faithfulness of the Dieudonné module functor over perfect rings (first deduced by Gabber from results of Berthelot, Ber80, cf. also [Lau13, Theorem D]), it is then enough to check that for any isocrystal $(D, \varphi)$ over $\overline{\mathbb{F}}_{p}$,

$$
(D \otimes W(R)[1 / p])^{\varphi=1}=(D \otimes W(k)[1 / p])^{\varphi=1} .
$$

We may assume that $D=D_{\lambda}$ is simple of slope $\lambda$; if $\lambda \neq 0$, then there are no $\varphi$-invariants, and if $\lambda=0$, then both sides are equal to $\mathbb{Q}_{p}$.

It remains to see that any $p$-divisible group $G$ over $R$ with constant Newton polygon is isogenous to a constant $p$-divisible group 22 More precisely, choose a completely slope divisible $G_{0} / \overline{\mathbb{F}}_{p}$ with an isogeny $\psi_{k}: G_{k} \rightarrow G_{0, k}$ which one can assume to be of degree bounded only in terms of the height $h$ of $G$. Then we claim that there is a (necessarily unique) quasi-isogeny $\psi: G \rightarrow G_{0, R}$ lifting $\psi_{k}$, and whose degree is bounded only in terms of $h$; i.e. there is a constant $c=c(h)$ such that $p^{c} \psi: G \rightarrow G_{0, R}$ is an isogeny.

For this, assume first that $R$ is an integral domain, with quotient field $K$. By Lemma 4.2.16 (cf. Remark 4.2.17), the functor from $p$-divisible groups over $R$ to $p$-divisible groups over $K$ is fully faithful. We can find an isogeny $\psi_{K}^{\prime}: G_{K} \rightarrow G_{0, K}$ of degree bounded only in terms of $h$, which then extends to a map $\psi^{\prime}: G \rightarrow G_{0, R}$ of degree bounded only in terms of $h$. Over $k, \psi_{k}$ and $\psi_{k}^{\prime}$ differ by a quasi-isogeny of $G_{0}$ of bounded degree; correcting $\psi^{\prime}$ by this quasi-isogeny gives the desired quasiisogeny $\psi: G \rightarrow G_{0, R}$ lifting $\psi_{k}$, which is of bounded degree.

In general, let $\left\{\mathfrak{p}_{i}\right\}$ be the minimal prime ideals of $R$ (which may be infinitely many) 23 then the result holds true over each $R / \mathfrak{p}_{i}$, which is still a strictly henselian perfect ring. Let $\tilde{R} \subset \prod_{i} R / \mathfrak{p}_{i}$ be the subring of those elements $f=\left\{f_{i} \in R / \mathfrak{p}_{i}\right\}$ for which $\bar{f}:=\bar{f}_{i} \in k$ is independent of $i$. Then $\tilde{R}$ is another strictly henselian perfect ring, $R \hookrightarrow \tilde{R}$, and there is an isogeny

$$
\psi_{\tilde{R}}: G_{\tilde{R}} \rightarrow G_{0, \tilde{R}} .
$$

Indeed, $p^{c} \psi_{\tilde{R}}$ will be an actual isogeny, and then to write down this isogeny, one has to write down many matrices with entries in $\tilde{R}$; but one has these matrices with entries in $R / \mathfrak{p}_{i}$ for each $i$, reducing to the same matrix over $k$. It remains to see that $\psi_{\tilde{R}}$ is defined over $R$, i.e. that some matrices with coefficients in $\tilde{R}$ have coefficients in $R$. For each $i, \tilde{R} / \mathfrak{p}_{i} \tilde{R}$ is a strictly henselian perfect ring, so $\psi_{\tilde{R} / \mathfrak{p}_{i} \tilde{R}}$ is uniquely determined; by uniqueness, it must be given by the base extension of $\psi_{R / \mathfrak{p}_{i}}$, which is already known to exist. Thus, we finish by observing that

$$
R=\left\{f \in \tilde{R} \mid \forall i: f \quad \bmod \mathfrak{p}_{i} \in R / \mathfrak{p}_{i} \subset \tilde{R} / \mathfrak{p}_{i} \tilde{R}\right\} .
$$

To verify the displayed equation, we observe that $R \rightarrow \tilde{R}$ is a $v$-cover in the sense of [BS15b], so that by [BS15b, Theorem 4.1 (i)] (applied to $\mathcal{E}=\mathcal{O}_{X}$ )

$$
R=\left\{f \in \tilde{R} \mid f \otimes 1=1 \otimes f \in \tilde{R} \otimes_{R} \tilde{R}\right\} .
$$

\footnotetext{
${ }^{22} \mathrm{Cf}$. OZ02, Corollary 3.6] in the case where $R$ is the perfection of a noetherian strictly henselian ring $R^{\prime}$ and $G$ is defined over $R^{\prime}$.

${ }^{23}$ If there are only finitely many, e.g. if $S$ is the perfection of a noetherian scheme, one can argue as in $\mathrm{OZ02}$ end of proof of Proposition 3.3].
} 
As everything is reduced, the latter equality can be checked as a system of equalities in

as desired.

$$
\left(\tilde{R} \otimes_{R} \tilde{R}\right) / \mathfrak{p}_{i}\left(\tilde{R} \otimes_{R} \tilde{R}\right)=\tilde{R} / \mathfrak{p}_{i} \tilde{R} \otimes_{R / \mathfrak{p}_{i}} \tilde{R} / \mathfrak{p}_{i} \tilde{R},
$$

Now we go back to a general perfect base scheme $S$. We need to find a quasiisogeny between $\mathbb{X}$ and $\mathbb{X}_{b}$ (compatible with extra structures) locally on $S_{\text {proét }}$. For any geometric point $\bar{x} \in S$, we can find such a quasi-isogeny over $S_{\bar{x}}$. Thus, fixing any $n$, after replacing $S$ by an étale neighborhood of $\bar{x}$ and $\mathbb{X}$ by a quasi-isogenous $p$-divisible group, we can assume that there is an isomorphism $\mathbb{X}\left[p^{n}\right] \cong \mathbb{X}_{b}\left[p^{n}\right]$ compatible with extra structure.

In that case, we can look at the $K_{b}$-quasitorsor $\tilde{S} \rightarrow S$ of isomorphisms $\mathbb{X}_{T} \cong$ $\left(\mathbb{X}_{b}\right)_{T}$ compatible with extra structures on the category of perfect $S$-schemes $T$, where $K_{b} \subset J_{b}\left(\mathbb{Q}_{p}\right)$ is the compact open subgroup of automorphisms of $\mathbb{X}_{b}$, compatible with extra structures. Note that $\tilde{S}$ is representable by a perfect scheme. We claim that if $n$ was chosen large enough (depending only on $\mathbb{X}_{b}$ ), then this quasitorsor is a torsor, i.e. $\tilde{S} \rightarrow S$ is faithfully flat. This will then give the desired quasi-isogeny locally on $S_{\text {proét }}$ (namely over the pro-étale cover $\tilde{S} \rightarrow S$ ).

To show that $\tilde{S}$ is a torsor, we need to see that it is faithfully flat, so we can assume that $S=\operatorname{Spec} R$ is strictly local. We need to show that there is an isomorphism $\mathbb{X} \cong\left(\mathbb{X}_{b}\right)_{R}$ compatible with extra structures, assuming that such an isomorphism exists on $p^{n}$-torsion for $n$ big enough.

As before, let $k$ be the residue field of $R$. Then $\mathbb{X}_{k}$ and $\mathbb{X}_{b}$ have isomorphic $p^{n}$ torsion; from [Sch13a, Lemma 4.4] one deduces that there is an isomorphism $\psi_{x}$ : $\mathbb{X}_{b} \cong \mathbb{X}_{k}$ compatible with extra structures, if $n$ was chosen large enough; moreover, one can assume that this isomorphism reduces to the given one $\mathbb{X}_{b}\left[p^{n}\right] \cong \mathbb{X}_{k}\left[p^{n}\right]$ on $p^{n / 2}$-torsion (say, $n=2 m$ is even). From Lemma 4.3.15 and Remark 4.3.16, we see that $\psi_{x}$ lifts to a quasi-isogeny $\psi:\left(\mathbb{X}_{b}\right)_{R} \rightarrow \mathbb{X}$, such that $p^{c} \psi:\left(\mathbb{X}_{b}\right)_{R} \rightarrow \mathbb{X}$ and $p^{c} \psi^{-1}: \mathbb{X} \rightarrow\left(\mathbb{X}_{b}\right)_{R}$ are actual isogenies, where $c$ is a constant depending only on $\mathbb{X}_{b}$. Then the kernel $G \subset\left(\mathbb{X}_{b}\right)_{R}$ of $p^{c} \psi$ is contained in the $p^{2 c}$-torsion; thus, it is the kernel of $p^{c} \psi:\left(\mathbb{X}_{b}\right)_{R}\left[p^{2 c}\right] \rightarrow \mathbb{X}\left[p^{2 c}\right] \cong\left(\mathbb{X}_{b}\right)_{R}\left[p^{2 c}\right]$ (if $m \geq 2 c$, which we may assume). By choosing $m$ large enough and using Lemma 4.1.5, we may arrange that $p^{c} \psi$ lies in $\mathcal{H}_{\mathbb{X}_{b}, \mathbb{X}_{b}}\left[p^{2 c}\right](R)$. But as $R$ is strictly henselian perfect,

$$
\mathcal{H}_{\mathbb{X}_{b}, \mathbb{X}_{b}}\left[p^{2 c}\right](R)=\mathcal{H}_{\mathbb{X}_{b}, \mathbb{X}_{b}}\left[p^{2 c}\right]\left(\overline{\mathbb{F}}_{p}\right) .
$$

It follows that $G \subset\left(\mathbb{X}_{b}\right)_{R}$ is constant, $G=G_{0, R}$, for $G_{0} \subset \mathbb{X}_{b}$, with $\mathbb{X}_{b} / G_{0} \cong \mathbb{X}_{b}$ compatibly with extra structures (as this is true over $k$ ). But then $p^{c} \psi$ factors over an isomorphism

$$
\left(\mathbb{X}_{b} / G_{0}\right)_{R} \cong \mathbb{X}
$$

where the left-hand side is isomorphic to $\left(\mathbb{X}_{b}\right)_{R}$. This gives the desired isomorphism $\mathbb{X} \cong\left(\mathbb{X}_{b}\right)_{R}$ compatible with extra structures.

Now we go back to the study of Igusa varieties. Let $\mathcal{X}^{b}:=\left(\mathfrak{X}^{b}\right)_{\eta}^{\text {ad }}$ be the adic generic fiber of the formal scheme $\mathfrak{X}^{b}$.

Definition 4.3.17. Let $\mathcal{X}_{\infty}^{b}$ be the functor on complete affinoid $\left(\breve{E}\left(\zeta_{p^{\infty}}\right), \mathcal{O}_{\breve{E}\left(\zeta_{p} \infty\right)}\right)$ algebras sending $\left(R, R^{+}\right)$to the set of triples $(\mathcal{A}, \rho, \alpha)$, where $(\mathcal{A}, \rho) \in \mathcal{X}^{b}\left(R, R^{+}\right)$ and

$$
\alpha: \Lambda \rightarrow T_{p} \mathcal{A}
$$


is a morphism of $\mathcal{O}_{B}$-modules such that

(1) the pairing $(\cdot, \cdot)$ on $\Lambda$ matches the pairing on $T_{p} \mathcal{A}$ induced by the polarization and the fixed choice of p-power roots of unity, and

(2) the induced maps

$$
\Lambda \rightarrow T_{p} \mathcal{A}_{\eta}^{\mathrm{ad}}\left(C, C^{+}\right),
$$

on all geometric points $\mathrm{Spa}\left(C, C^{+}\right)$of $\mathrm{Spa}\left(R, R^{+}\right)$are isomorphisms.

Remark 4.3.18. There are natural maps $\mathfrak{X}^{b} \rightarrow \mathfrak{M}^{b}$ and $\mathcal{X}_{\infty}^{b} \rightarrow \mathcal{M}_{\infty}^{b}$, defined by sending an abelian variety to its $p$-divisible group. We can check on the level of moduli problems that $\mathcal{X}_{\infty}^{b}$ fits into the Cartesian diagram

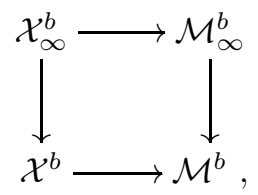

therefore it is representable by an adic space.

We let $\left(\operatorname{Ig}_{\mathcal{O}_{K}}^{b}\right)_{\eta}^{\text {ad }}$ be the generic fiber of the formal scheme $\operatorname{Ig}_{\mathcal{O}_{K}}^{b}$.

Corollary 4.3.19. We have an isomorphism

$$
\left(\operatorname{Ig}_{\mathcal{O}_{K}}^{b}\right)_{\eta}^{\text {ad }} \times_{\operatorname{Spa}\left(\breve{E}, \mathcal{O}_{\breve{E}}\right)} \mathcal{M}_{\infty}^{b} \stackrel{\sim}{\rightarrow} \mathcal{X}_{\infty, K}^{b}
$$

In particular, $\mathcal{X}_{\infty}^{b}$ is preperfectoid.

Proof. The first part follows from the decomposition of $\mathfrak{X}^{b}$ in Lemma4.3.12, and the cartesian diagram of Remark 4.3.18. The final assertion follows formally from the facts that $\mathcal{M}_{\infty}^{b}$ is preperfectoid, and that $\operatorname{Ig}_{\mathcal{O}_{K}}^{b}$ is locally of the form $W(R) \otimes_{\mathcal{O}_{\breve{E}}} \mathcal{O}_{K}$ for a perfect ring $R$, so that (if $K$ is perfectoid) its generic fibre is a perfectoid space.

We let $\widehat{\mathcal{X}}_{\infty}^{b}$ be the perfectoid space associated with $\mathcal{X}_{\infty}^{b}$ as in SW13, Proposition 2.3.6]. Let $\mathcal{S}_{K^{p}}$ be the perfectoid infinite-level Shimura variety over $E_{\mathfrak{p}}$. Let $\mathcal{S}_{K^{p}}^{b} \subset$ $\mathcal{S}_{K^{p}}$ be the locus of those points $\operatorname{Spa}\left(K, K^{+}\right) \rightarrow \mathcal{S}_{K^{p}}$ over which the universal abelian variety over $K$ extends to $K^{+}$, and defines a point of $\mathscr{S}_{K_{p} K^{p}}^{b}$ over the residue field of $K^{+}$. This is the preimage under the continuous specialization map of the locally closed subset $\mathscr{S}_{K_{p} K^{p}}^{b} \subset \mathscr{S}_{K_{p} K^{p}} \times_{\mathcal{O}_{E, \mathfrak{p}}} \mathbb{F}_{q}$, and thus $\mathcal{S}_{K^{p}}^{b} \subset \mathcal{S}_{K^{p}}$ is a locally closed subset.

Lemma 4.3.20. The perfectoid space $\widehat{\mathcal{X}}^{b}$ maps to $\mathcal{S}_{K^{p}}^{b}$ by forgetting the quasiisogeny $\rho$ and to $\mathcal{M}_{\infty}^{b}$ by sending $(\mathcal{A}, \rho)$ to $\left(\mathcal{A}\left[p^{\infty}\right], \rho\right)$. The induced map

$$
\widehat{\mathcal{X}}_{\infty}^{b} \rightarrow\left(\mathcal{M}_{\infty}^{b} \times \mathscr{F}_{G, \mu} \mathcal{S}_{K^{p}}^{b}\right)^{\wedge}
$$

is an isomorphism of perfectoid spaces.

In other words,

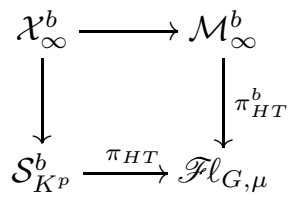

becomes a Cartesian diagram when one takes points over a perfectoid space. 
Proof. Note that the diagram commutes by Remark 4.2.8, Therefore, the map in the lemma is well-defined. We first check the fact that the diagram is Cartesian on $\left(C, \mathcal{O}_{C}\right)$-points, where $C / \breve{E}\left(\zeta_{p^{\infty}}\right)$ is complete and algebraically closed with ring of integers $\mathcal{O}_{C}$. A $\left(C, \mathcal{O}_{C}\right)$-point of $\mathcal{S}_{K^{p}}^{b}$ gives rise to a couple $(\mathcal{A}, \alpha)$, where $\mathcal{A} / \mathcal{O}_{C}$ is an abelian variety with extra structures and $\alpha: \Lambda \rightarrow T_{p} \mathcal{A}\left(C, \mathcal{O}_{C}\right)$ is an isomorphism compatible with extra structures. A $\left(C, \mathcal{O}_{C}\right)$-point of $\mathcal{M}_{\infty}^{b}$ gives us a triple $(\mathcal{G}, \beta, \rho)$, where $\mathcal{G} / \mathcal{O}_{C}$ is a $p$-divisible group with extra structures, $\beta: \Lambda \stackrel{\sim}{\rightarrow} T_{p} \mathcal{G}\left(C, \mathcal{O}_{C}\right)$ is a trivialization of its integral Tate module and $\mathcal{G} \times_{\mathcal{O}_{C}} \mathcal{O}_{C} / p \rightarrow \mathbb{X}_{b} \times_{\overline{\mathbb{F}}_{p}} \mathcal{O}_{C} / p$ is a quasi-isogeny.

The fact that $(\mathcal{A}, \alpha)$ and $(\mathcal{G}, \beta, \rho)$ are mapped to the same point of $\mathscr{F} \ell_{G, \mu}$ under $\pi_{H T}$ and $\pi_{H T}^{b}$ means that the Hodge-Tate filtrations on $T_{p} \mathcal{A} \otimes C$ and $T_{p} \mathcal{G} \otimes C$ are identified under the isomorphism $\beta \circ \alpha^{-1}$. Now [SW13, Theorem B] gives an isomorphism $\mathcal{A}\left[p^{\infty}\right] \cong \mathcal{G}$ extending the given isomorphism on the generic fibre. Thus, the given data assemble into a point of $\mathcal{X}_{\infty}^{b}$, and one checks that these constructions are inverse.

Now, if $\left(R, R^{+}\right)$is any perfectoid affinoid $\breve{E}\left(\zeta_{p^{\infty}}\right)$-algebra, one gets similar data $(\mathcal{A}, \alpha),(\mathcal{G}, \beta, \rho)$ over $R^{+}$. One has to check that the isomorphism $\beta \circ \alpha^{-1}$ between $\mathcal{A}\left[p^{\infty}\right]_{R}$ and $\mathcal{G}_{R}$ extends to $R^{+}$. This follows from Lemma 4.2.15 above.

Putting together Remark 4.3.18 and Lemma 4.3.20 we get a diagram with Cartesian squares (the right one when evaluated on perfectoid spaces)

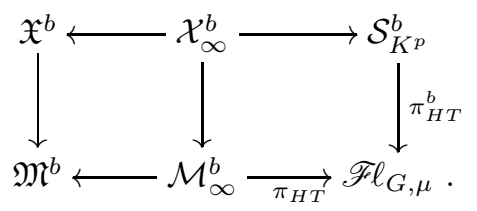

4.4. Étale cohomology. Fix a prime $\ell \neq p$, and consider the map

$$
\pi_{H T}: \mathcal{S}_{K^{p}} \rightarrow \mathscr{F} \ell_{G, \mu} .
$$

In this final subsection, we use the geometric results established so far to identify the fibres of $\mathcal{F}=R \pi_{H T *} \mathbb{Z} / \ell^{n} \mathbb{Z}$ with the cohomology of Igusa varieties. In this section, we make the additional assumption that $\mathscr{S}_{K_{p} K^{p}}$ is proper over $\mathcal{O}_{E, \mathfrak{p}}$. It is known that this is equivalent to asking that $G$ is anisotropic over $\mathbb{Q}$, cf. [Lan11.

Let $C$ be a complete algebraically closed extension of $\breve{E}\left(\zeta_{p^{\infty}}\right)$, with an open and bounded valuation subring $C^{+} \subset C$, and fix a point $x \in \mathscr{F} \ell_{G, \mu}\left(C, C^{+}\right)$; we assume that $C$ is the completed algebraic closure of the residue field of $\mathscr{F} \ell_{G, \mu}$ at the underlying (topological) point. We are interested in understanding the stalk $\mathcal{F}_{x}=\left(R^{i} \pi_{H T *} \mathbb{Z} / \ell^{n} \mathbb{Z}\right)_{x}$. In this respect, we have the following general base change lemma.

Lemma 4.4.1. Let $f: Y \rightarrow X$ be a quasicompact and quasiseparated map of analytic adic spaces, and for definiteness assume that $X$ is either a locally strongly noetherian adic space or a perfectoid space over $\mathrm{Spa}\left(\mathbb{Z}_{p}, \mathbb{Z}_{p}\right)$, and $Y$ is perfectoid 24 Let $x \in X$ be a point with residue field $K(x)$ and open and bounded valuation subring $K(x)^{+}$. Let $C(\bar{x})$ be a completed algebraic closure of $K(x)$ with an open and

\footnotetext{
${ }^{24}$ We only need to know that they have well-defined étale sites, and that the same holds for all fibres of $f$ over geometric points. For example, the lemma is also true when one asssumes instead that both $X$ and $Y$ are perfectoid.
} 
bounded valuation subring $C(\bar{x})^{+} \subset C(\bar{x})$ lifting $K(x)^{+}$, giving rise to a geometric point $\bar{x}=\operatorname{Spa}\left(C(\bar{x}), C(\bar{x})^{+}\right) \rightarrow X$. Let

$$
Y_{\bar{x}}=\left(Y \times_{X} \operatorname{Spa}\left(C(\bar{x}), C(\bar{x})^{+}\right)\right)^{\wedge}
$$

be the fibre of $Y$ over $\bar{x}$, which is a perfectoid space over $C(\bar{x})$. For any sheaf $\mathcal{G}$ of abelian groups on $Y_{\text {ét }}$ and all $i \geq 0$, the natural map

$$
\left(R^{i} f_{*} \mathcal{G}\right)_{\bar{x}} \rightarrow H^{i}\left(Y_{\bar{x}}, \mathcal{G}\right)
$$

is an isomorphism.

Here, and in the following, these statements will also be true for sheaves of groups and $i=0,1$, and sheaves of sets and $i=0$. We will not spell this out.

Proof. Let $U_{j}=\operatorname{Spa}\left(R_{j}, R_{j}^{+}\right) \rightarrow X$ be a cofinal system of affinoid étale neighborhoods of $\bar{x}$; then

$$
\operatorname{Spa}\left(C(x), C(x)^{+}\right) \sim \lim _{j} \operatorname{Spa}\left(R_{j}, R_{j}^{+}\right)
$$

and one has

$$
\left(R^{i} f_{*} \mathcal{G}\right)_{\bar{x}}=\underset{j}{\lim } H^{i}\left(Y \times_{X} \operatorname{Spa}\left(R_{j}, R_{j}^{+}\right), \mathcal{G}\right) .
$$

It remains to see that

$$
\underset{j}{\lim _{\vec{j}}} H^{i}\left(Y \times_{X} \operatorname{Spa}\left(R_{j}, R_{j}^{+}\right), \mathcal{G}\right)=H^{i}\left(Y_{\bar{x}}, \mathcal{G}\right) .
$$

But this follows from $Y_{\bar{x}} \sim \lim _{j} Y \times_{X} \operatorname{Spa}\left(R_{j}, R_{j}^{+}\right)$(cf. [SW13, Proposition 2.4.3]), where all terms are quasicompact and quasiseparated, and the resulting consequence for étale cohomology, cf. [Sch12a, Corollary 7.18] 25

In particular, the fibre

$$
\left(R^{i} \pi_{H T *} \mathbb{Z} / \ell^{n} \mathbb{Z}\right)_{x}=H^{i}\left(\mathcal{S}_{K^{p}, x}, \mathbb{Z} / \ell^{n} \mathbb{Z}\right) .
$$

Next, we reduce to the case of rank 1 points. For this, we use the following lemma.

Lemma 4.4.2. Let $X$ be a quasicompact and quasiseparated analytic adic space, and for definiteness assume that $X$ is a perfectoid space ${ }^{26}$ Let $U \subset X$ be a quasicompact open subset which contains all rank 1 points of $X$. Then, for any locally constant sheaf $\mathcal{G}$ of abelian groups on $X_{\text {ét }}$ and all $i \geq 0$, the natural map

$$
H^{i}(X, \mathcal{G}) \rightarrow H^{i}(U, \mathcal{G})
$$

is an isomorphism.

Proof. Let $j: U \hookrightarrow X$ be the inclusion. It is enough to prove that $\mathcal{G} \rightarrow R j_{*} \mathcal{G}$ is an isomorphism. This can be checked on geometric points, which, using Lemma 4.4.1, reduces us to the case $X=\operatorname{Spa}\left(C, C^{+}\right)$for some complete algebraically closed field $C$ with an open and bounded valuation subring $C^{+} \subset C$. Then $U=\operatorname{Spa}\left(C, D^{+}\right)$ for a different open and bounded valuation subring $D^{+} \subset C$, containing $C^{+}$. As $X$ is strictly local, the sheaf $\mathcal{G}$ is the constant sheaf associated with some abelian group $G$. But as any étale cover of $X$ splits, one has $R \Gamma(X, G)=G$, and similarly for $U$, giving the result.

\footnotetext{
${ }^{25}$ In the discussion around Sch12a Corollary 7.18], the $X_{i}$ are assumed to be strongly noetherian; the discussion is also valid if all $X_{i}$ are perfectoid.

${ }^{26}$ Again, the lemma also holds true when $X$ is a strongly noetherian adic space, or whenever $X$ has a well-behaved étale site.
} 
Applying Lemma 4.4.2 to the inclusion $\mathcal{S}_{K^{p}, \tilde{x}} \subset \mathcal{S}_{K^{p}, x}$ shows that

$$
\left(R^{i} \pi_{H T *} \mathbb{Z} / \ell^{n} \mathbb{Z}\right)_{x}=\left(R^{i} \pi_{H T *} \mathbb{Z} / \ell^{n} \mathbb{Z}\right)_{\tilde{x}}=H^{i}\left(\mathcal{S}_{K^{p}, \tilde{x}}, \mathbb{Z} / \ell^{n} \mathbb{Z}\right) .
$$

Thus, we will from now on assume that $x=\tilde{x}$ is a rank 1 point, and write $C^{+}=\mathcal{O}_{C}$. Now choose $b \in B\left(G, \mu^{-1}\right)$ such that $x \in \mathscr{F}_{G, \mu}^{b}$. If $y \in \mathcal{S}_{K^{p}, x}$ is any (geometric) rank 1 point, the argument of Lemma 4.2.18 shows that $y \in \mathcal{S}_{K^{p}}^{b}$. Thus, $\mathcal{S}_{K^{p}, x}^{b}=$ $\mathcal{S}_{K^{p}, x} \times_{\mathcal{S}_{K^{p}}} \mathcal{S}_{K^{p}}^{b} \subset \mathcal{S}_{K^{p}, x}$ is a quasicompact open subset with the same rank 1 points, so applying Lemma 4.4 .2 once more, we see that

$$
\left(R^{i} \pi_{H T *} \mathbb{Z} / \ell^{n} \mathbb{Z}\right)_{x}=H^{i}\left(\mathcal{S}_{K^{p}, x}^{b}, \mathbb{Z} / \ell^{n} \mathbb{Z}\right) .
$$

Now we apply Lemma 4.2 .18 to lift $x \in \mathscr{F}_{G, \mu}^{b}\left(C, \mathcal{O}_{C}\right)$ to a point $z \in \mathcal{M}_{\infty}^{b}\left(C, \mathcal{O}_{C}\right)$, giving rise in particular to a $p$-divisible group $\left(\mathbb{X}_{b}\right)_{\mathcal{O}_{C}}$ (with extra structures) lifting $\mathbb{X}_{b}$. Then Lemma 4.3 .20 identifies the fibre $\mathcal{S}_{K^{p}, x}^{b}$ with the fibre $\mathcal{X}_{\infty, z}^{b}$. This, in turn, gets identified with $\left(\operatorname{Ig}_{\mathcal{O}_{C}}^{b}\right)_{\eta}^{\text {ad }}$ by Corollary 4.3.19, Combining the discussion so far, we see that

$$
\left(R^{i} \pi_{H T *} \mathbb{Z} / \ell^{n} \mathbb{Z}\right)_{x}=H^{i}\left(\left(\operatorname{Ig}_{\mathcal{O}_{C}}^{b}\right)_{\eta}^{\mathrm{ad}}, \mathbb{Z} / \ell^{n} \mathbb{Z}\right) .
$$

Next, we pass to the special fibre.

Lemma 4.4.3. Let $X / \overline{\mathbb{F}}_{p}$ be a perfect scheme and let $C$ be a complete algebraically closed nonarchimedean field whose residue field contains $\overline{\mathbb{F}}_{p}$. Let $\mathfrak{X}_{\mathcal{O}_{C}}$ be the flat formal scheme over Spf $\mathcal{O}_{C}$ which is the unique lifting of $X \times_{\overline{\mathbb{F}}_{p}} \mathcal{O}_{C} / p$, and let $\mathcal{X}_{C}=\left(\mathfrak{X}_{\mathcal{O}_{C}}\right)_{\eta}^{\text {ad }}$ be its generic fibre, which is a perfectoid space. For all $i$, the canonical maps

$$
H^{i}\left(X, \mathbb{Z} / \ell^{n} \mathbb{Z}\right) \leftarrow H^{i}\left(\mathfrak{X}_{\mathcal{O}_{C}}, \mathbb{Z} / \ell^{n} \mathbb{Z}\right) \rightarrow H^{i}\left(\mathcal{X}_{C}, \mathbb{Z} / \ell^{n} \mathbb{Z}\right)
$$

are isomorphisms.

Proof. The question is local on $X$, so we can assume that $X$ is affine. Then we can write $X=\lim X_{j}$ as a cofiltered inverse limit of affine schemes $X_{j}$ which are perfections of schemes of finite type over $\overline{\mathbb{F}}_{p}$. One also gets $\mathcal{X}_{C} \sim \lim _{j} \mathcal{X}_{j, C}$, so all cohomology groups in question become a filtered colimit over $j$; thus, we can assume that $X$ is the perfection of an affine scheme $X_{0}$ of finite type. Then the cohomology of $X$ agrees with the cohomology of $X_{0}$.

Moreover, the cohomology of $\mathfrak{X}_{\mathcal{O}_{C}}$ is the same as the cohomology of its special fibre $X \times_{\overline{\mathbb{F}}_{p}} k$, where $k$ is the residue field of $\mathcal{O}_{C}$, which in turn agrees with the cohomology of $X_{0} \times_{\overline{\mathbb{F}}_{p}} k$. Thus, the first map is an isomorphism by invariance of étale cohomology under change of algebraically closed base field.

Also, under tilting, the étale cohomology of $\mathcal{X}_{C}$ agrees with the étale cohomology of $\mathcal{X}_{C^{b}}$. We may thus assume that $C$ is of characteristic $p$. In that case, one can also form $\mathfrak{X}_{0, \mathcal{O}_{C}}=X_{0} \times_{\text {Spec }} \overline{\mathbb{F}}_{p}$ Spf $\mathcal{O}_{C}$ and its generic fibre $\mathcal{X}_{0, C}$, which is a rigidanalytic variety over $C$, with $\mathcal{X}_{C} \sim \lim _{\text {Frob }} \mathcal{X}_{0, C}$. Thus, the cohomology of $\mathcal{X}_{C}$ agrees with the cohomology of $\mathcal{X}_{0, C}$. Finally, we are reduced to proving that the map

$$
H^{i}\left(\mathfrak{X}_{0, \mathcal{O}_{C}}, \mathbb{Z} / \ell^{n} \mathbb{Z}\right) \rightarrow H^{i}\left(\mathcal{X}_{0, C}, \mathbb{Z} / \ell^{n} \mathbb{Z}\right)
$$

is an isomorphism. The right hand side can be computed, by Hub96, Corollary 3.5.17], in terms of $H^{i-j}\left(X_{0} \times_{\overline{\mathbb{F}}_{p}} k, R^{j} \psi \mathbb{Z} / \ell^{n} \mathbb{Z}\right)$.

It is enough to see that, if $X_{0}$ is a scheme of finite type over $\overline{\mathbb{F}}_{p}$, then the complex of nearby cycles of $X_{0, C}=X_{0} \times_{\overline{\mathbb{F}}_{p}} C$ is quasi-isomorphic to the constant sheaf $\mathbb{Z} / \ell^{n} \mathbb{Z}$. 
By [SGA73, XIII 2.1.4], we can compute the stalk of $R^{j} \psi \mathbb{Z} / \ell^{n} \mathbb{Z}$ at a geometric point $\bar{x}$ as $H^{j}\left(\left(X_{0, \mathcal{O}_{C}}\right)_{\bar{x}} \times C, \mathbb{Z} / \ell^{n} \mathbb{Z}\right)$, with $\left(X_{0, \mathcal{O}_{C}}\right)_{\bar{x}}$ the strict henselization of $X_{0, \mathcal{O}_{C}}=X_{0} \times_{\overline{\mathbb{F}}_{p}} \mathcal{O}_{C}$ at $\bar{x}$. We conclude, since the map $X_{0, C} \rightarrow$ Spec $\mathcal{O}_{C}$ is the base change along the map Spec $\mathcal{O}_{C} \rightarrow$ Spec $\overline{\mathbb{F}}_{p}$ of the universally locally acyclic map $X_{0} \rightarrow \overline{\mathbb{F}}_{p}$. (For universal local acyclicity, we use the definition of Del77]. Every scheme of finite type is universally locally acyclic over a point, cf. Del77, Th. finitude, Théorème 2.13].)

Thus, we get

$$
\left(R^{i} \pi_{H T *} \mathbb{Z} / \ell^{n} \mathbb{Z}\right)_{x}=H^{i}\left(\operatorname{Ig}^{b}, \mathbb{Z} / \ell^{n} \mathbb{Z}\right)
$$

where $\operatorname{Ig}^{b} / \overline{\mathbb{F}}_{p}$ is the perfect scheme introduced in Definition 4.3.1 Using Proposition 4.3.8, we finally arrive at the following formula.

Theorem 4.4.4. For any geometric point $\bar{x}$ of $\mathscr{F} \ell_{G, \mu}$ contained in $\mathscr{F} \ell_{G, \mu}^{b}$, there is an isomorphism

$$
\left(R^{i} \pi_{H T *} \mathbb{Z} / \ell^{n} \mathbb{Z}\right)_{\bar{x}}=H^{i}\left(\operatorname{Ig}^{b}, \mathbb{Z} / \ell^{n} \mathbb{Z}\right)=\underset{m}{\lim } H^{i}\left(\mathscr{I}_{\text {Mant }, m}^{b}, \mathbb{Z} / \ell^{n} \mathbb{Z}\right) .
$$

It (only) depends on the choice of a lift of $\bar{x}$ to $\mathcal{M}_{\infty}^{b}$, and is compatible with the Hecke action of $G\left(\mathbb{A}_{f}^{p}\right)$.

One can formulate a version of this result where one replaces $\mathbb{Z} / \ell^{n} \mathbb{Z}$ by the local system corresponding to an algebraic representation $\xi$. 


\section{The COHOMOlogy of Igusa VARIETIES}

The goal of this section is to compute the alternating sum of cohomology groups $\left[H\left(\mathfrak{I g}^{b}, \overline{\mathbb{Q}}_{\ell}\right)\right]$ as a virtual representation of $G\left(\mathbb{A}_{f}^{p}\right) \times J_{b}\left(\mathbb{Q}_{p}\right)$. We will work with (the Igusa varieties corresponding to) unitary Shimura varieties. Our setup is similar to that of [SS13] (see Section 5.1 for more detail) and we intend to prove a version of Theorem 6.1 of Shi11 in this situation.

By Proposition 4.3.8 and since perfection does not change the étale topos, it is enough to work with the classical objects $\mathscr{I}_{\text {Mant }}^{b}$. By Poincaré duality, it is enough to compute the alternating sum of the compactly supported cohomology groups. Sug Woo Shin has derived a formula for the alternating sum $\left[H_{c}\left(\mathscr{I}_{\text {Mant }}^{b}, \overline{\mathbb{Q}}_{\ell}\right)\right]$ as a sum of stable orbital integrals for $G$ and its elliptic endoscopic groups (see Theorem 5.2.3). We reinterpret this formula as the geometric side of the twisted trace formula and compare it to the spectral side.

5.1. Setup. We assume that $F=F^{+} \cdot \mathcal{K}$ is the composition of a totally real field $F^{+}$ and an imaginary quadratic field $\mathcal{K}$. Let $c \in \operatorname{Gal}\left(F / F^{+}\right)$be the non-trivial element. Let $G / \mathbb{Q}$ be a unitary similitude group preserving an alternating hermitian form $\langle$,$\rangle on an F$-vector space $V$ of dimension $n$. Let $\mathrm{Spl}_{F / F^{+}}$denote the set of rational primes $v$ such that every prime of $F^{+}$above $v$ splits in $F$. We make the following further assumptions on $F$ and $G$.

(1) $F^{+} \neq \mathbb{Q}$;

(2) the set of rational primes which are ramified in $F$ is contained in $\mathrm{Spl}_{F / F^{+}}$;

(3) $G$ is quasi-split at all finite places.

See Section 10 of $S S 13$ for a discussion of these conditions. The first two are imposed to avoid issues with $L$-packets and base change for unitary groups 27 The

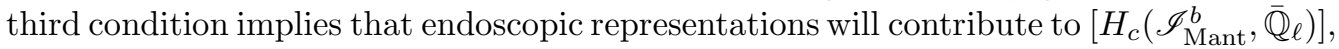
and is thus in some sense the hardest case.

Let $h: \mathbb{C} \rightarrow \operatorname{End}_{F}(V)_{\mathbb{R}}$ be an $\mathbb{R}$-algebra homomorphism such that $h\left(z^{c}\right)=h(z)^{c}$ for all $z \in \mathbb{C}$ and such that the bilinear pairing $(v, w) \mapsto\langle v, h(i) w\rangle$ is symmetric and positive definite. Then $(F, c, V,\langle\rangle, h$,$) is a Shimura datum of PEL type. The$ fact that $c$ is an involution of the second kind implies that the PEL datum is of type $(A)$, according to the classification on page 375 of Kot85.

The $\mathbb{R}$-algebra homomorphism $h$ induces a homomorphism of algebraic groups $h: \operatorname{Res}_{\mathbb{C} / \mathbb{R}} \mathbb{G}_{m} \rightarrow G_{\mathbb{R}}$. Then $(G,\{h\})$ is a Shimura datum as in Section 2.1 For $K \subset$ $G\left(\mathbb{A}_{f}\right)$ an open compact subgroup, we can define the Shimura variety $S_{K}$, which has a model over the reflex field $E$. Let $\mu$ be the Hodge cocharacter corresponding to $h$. We follow the slight abuse of notation in denoting by $S_{K}$ not the actual Shimura variety, but the PEL moduli problem, which is the disjoint union of $\left|\operatorname{ker}^{1}(G, \mathbb{Q})\right|$ copies of the actual Shimura variety. This factor $\left|\operatorname{ker}^{1}(\mathbb{Q}, G)\right|$ will thus appear in many formulas below.

Also assume that the prime $p$ is unramified in $F$ and splits in $\mathcal{K}$ (so, in particular, it lies in $\left.\mathrm{Spl}_{F / F^{+}}\right)$.

Let $\mathfrak{p}$ be a prime in the reflex field $E$ of the Shimura datum above the rational prime $p$. Let $K \subset G\left(\mathbb{A}_{f}\right)$ be a compact open subgroup which is sufficiently small and has the form $K^{p} K_{p}$, such that $K_{p} \subset G\left(\mathbb{Q}_{p}\right)$ is hyperspecial. The fact that $p$ is unramified in $F$ means that good integral models $\mathscr{S}_{K}$ of $S_{K}$ exist over $\mathcal{O}_{E_{\mathfrak{p}}}$.

\footnotetext{
${ }^{27}$ Actually, (2) implies (1).
} 
We fix a field isomorphism $\iota_{\ell}: \overline{\mathbb{Q}}_{\ell} \stackrel{\sim}{\rightarrow} \mathbb{C}$ throughout. If $\mathrm{G}$ is a topological group, such that every neighborhood of the identity contains a compact-open subgroup and $\Omega$ is an algebraically closed field of characteristic 0 , we let $C_{c}^{\infty}(\mathrm{G})$ be the space of smooth, compactly supported, $\Omega$-valued functions on $\mathrm{G}$ (usually they will be $\mathbb{C}$-valued; if they are valued in $\overline{\mathbb{Q}}_{\ell}$, then by smooth we mean locally-constant). We let $\operatorname{Irr}(\mathrm{G})$ denote the set of isomorphism classes of irreducible admissible representations of $\mathrm{G}$ over $\Omega$ and $\operatorname{Groth}(\mathrm{G})$ be the corresponding Grothendieck group. For all the groups we consider, we choose Haar measures and transfer factors as in Shi10, Shi11.

In particular, if $\mathrm{G}$ is an unramified group over a non-archimedean field $\mathrm{F}$, we choose a hyperspecial maximal compact subgroup $\mathrm{K}$ and a Haar measure such that $\mathrm{K}$ has volume 1 . We let $\mathcal{H}^{\mathrm{ur}}(\mathrm{G}(\mathrm{F}))$ be the subspace of $C_{c}^{\infty}(\mathrm{G}(\mathrm{F}))$ consisting of bi-K-invariant functions, which is an algebra with respect to convolution.

5.2. A stable trace formula. In this section, we recall the main constructions and results of [Shi10]. For any open compact subgroup $K \subset G\left(\mathbb{A}_{f}\right)$ which is hyperspecial at $p$ we have an integral model $\mathscr{S}_{K} / \mathcal{O}_{E_{\mathfrak{p}}}$. As described in Section 4.3 , the special fiber of $\mathscr{S}_{K}$ has a Newton polygon stratification, in terms of elements $b \in B\left(G, \mu^{-1}\right)$. Fix $b$ and also a $p$-divisible group with extra structures $\mathbb{X}_{b} / \overline{\mathbb{F}}_{p}$ as in Section 4.3. Recall that $J_{b}\left(\mathbb{Q}_{p}\right)$ is the group of quasi-self-isogenies of $\mathbb{X}_{b}$ which respect all the extra structures.

By the Igusa variety $\mathscr{I}_{\text {Mant }}^{b}$ we mean the projective system of $\overline{\mathbb{F}}_{p^{-}}$schemes $\mathscr{I}_{\text {Mant }, K^{p}, m}^{b}$, where $K^{p} \subset G\left(\mathbb{A}_{f}^{p}\right)$ runs over sufficiently small open compact subgroups and $m$ runs over positive integers. Each of these schemes is a finite Galois cover of the leaf $\mathscr{C}_{b}$ inside $\mathscr{S}_{K}^{b}$. Define

$$
\left[H_{c}\left(\mathscr{I}_{\text {Mant }}^{b}, \overline{\mathbb{Q}}_{\ell}\right)\right]:=\bigoplus_{k}(-1)^{k} \underset{K^{p}, m}{\lim _{c}} H_{c}^{k}\left(\mathscr{I}_{\text {Mant }, K^{p}, m}^{b}, \overline{\mathbb{Q}} \ell_{\ell}\right) .
$$

Since each of the summands is an admissible representation of $G\left(\mathbb{A}_{f}^{p}\right) \times J_{b}\left(\mathbb{Q}_{p}\right)$, we think of $\left[H_{c}\left(\mathscr{I}_{\text {Mant }}^{b}, \overline{\mathbb{Q}}_{\ell}\right)\right]$ as a virtual representation in $\operatorname{Groth}\left(G\left(\mathbb{A}_{f}^{p}\right) \times J_{b}\left(\mathbb{Q}_{p}\right)\right)$.

Often, we will fix a finite set $S$ of places of $\mathbb{Q}$ including $p, \infty$ and all places at which $F$ ramifies. If we fix a compact open subgroup $K^{S} \subset G\left(\mathbb{A}^{S}\right)$ which is a product of hyperspecial maximal compact open subgroups $K_{q} \subset G\left(\mathbb{Q}_{q}\right)$, we let

$$
\left[H_{c}\left(\mathscr{I}_{\text {Mant }}^{b}, \overline{\mathbb{Q}}_{\ell}\right)\right]^{\text {Sur }}
$$

be the summand of $\left[H_{c}\left(\mathscr{I}_{\text {Mant }}^{b}, \overline{\mathbb{Q}}_{\ell}\right)\right]$ of those representations which are unramified outside $S$. More precisely, any element $\pi \in \operatorname{Groth}\left(G\left(\mathbb{A}_{f}^{p}\right) \times J_{b}\left(\mathbb{Q}_{p}\right)\right)$ can be written as a (possibly infinite) sum

$$
\pi=\sum_{i} n_{i} \pi_{i}
$$

where $\pi_{i}$ runs through the irreducible representations of $G\left(\mathbb{A}_{f}^{p}\right) \times J_{b}\left(\mathbb{Q}_{p}\right)$ (all of which decompose into a tensor product), $n_{i} \in \mathbb{Z}$, and for each compact open subgroup $K \subset G\left(\mathbb{A}_{f}^{p}\right) \times J_{b}\left(\mathbb{Q}_{p}\right)$, there are only finitely many $i$ for which $n_{i} \neq 0$ and $\pi_{i}^{K} \neq 0$. Then we define

$$
\pi^{S \text { ur }}=\sum_{i: \pi_{i}^{K^{S}} \neq 0} n_{i} \pi_{i}
$$

Let $\operatorname{Groth}\left(G\left(\mathbb{A}_{f}^{p}\right) \times J_{b}\left(\mathbb{Q}_{p}\right)\right)^{S \text { ur }}$ denote the subgroup of $\operatorname{Groth}\left(G\left(\mathbb{A}_{f}^{p}\right) \times J_{b}\left(\mathbb{Q}_{p}\right)\right)$ consisting of those $\pi$ for which $\pi=\pi^{S \text { ur }}$. Then there are nondegenerate trace 
pairings

and

$$
\operatorname{Groth}\left(G\left(\mathbb{A}_{f}^{p}\right) \times J_{b}\left(\mathbb{Q}_{p}\right)\right) \times C_{c}^{\infty}\left(G\left(\mathbb{A}_{f}^{p}\right) \times J_{b}\left(\mathbb{Q}_{p}\right)\right) \rightarrow \mathbb{C},
$$

$\operatorname{Groth}\left(G\left(\mathbb{A}_{f}^{p}\right) \times J_{b}\left(\mathbb{Q}_{p}\right)\right)^{S \text { ur }} \times\left(\mathcal{H}^{\mathrm{ur}}\left(G\left(\mathbb{A}^{S}\right)\right) \otimes C_{c}^{\infty}\left(G\left(\mathbb{A}_{S_{\text {fin }} \backslash\{p\}}\right) \times J_{b}\left(\mathbb{Q}_{p}\right)\right)\right) \rightarrow \mathbb{C}$.

Let $\phi \in C_{c}^{\infty}\left(G\left(\mathbb{A}_{f}^{p}\right) \times J_{b}\left(\mathbb{Q}_{p}\right)\right)$. We say that $\phi$ is acceptable if it satisfies the conditions of Definition 6.2 of Shi09. The main condition is that $\phi$ is a linear combination of functions of the form $\phi^{p} \times \phi_{p}$, where $\phi_{p}$ is supported on $\nu_{b}$-acceptable elements of $J_{b}\left(\mathbb{Q}_{p}\right)$. These are those elements $\delta \in J_{b}\left(\mathbb{Q}_{p}\right), \delta=\left(\delta_{i}\right) \in \prod_{i=1}^{r} \mathscr{A} u t^{0}\left(\mathbb{X}_{i}\right)$, such that any eigenvalues $e_{i}$ of $\delta_{i}$ satisfy

$$
v_{p}\left(e_{i}\right)<v_{p}\left(e_{j}\right) \text { whenever } \lambda_{i}>\lambda_{j}
$$

(Definition 6.1 of [Shi09]).

Remark 5.2.1. This condition will separate components of $J_{b}\left(\mathbb{Q}_{p}\right)$ corresponding to different slopes in terms of their $p$-adic valuation, which in turn is needed in order to transfer functions from $J_{b}\left(\mathbb{Q}_{p}\right)$ to $G\left(\mathbb{Q}_{p}\right)$. See Lemma 3.9 of [Shi10] and Lemma V.5.2 of [HT01] for more details.

Lemma 6.3 of [Shi09] guarantees that the twist of any $\phi$ by a sufficiently high power of Frobenius is acceptable.

We recall the $\operatorname{set} \mathcal{E}^{\text {ell }}(G)$ of elliptic endoscopic triples for $G$. In fact, we work more generally: let $\mathrm{F}$ be a local or global field of characteristic 0 and let $\mathrm{G}$ be a connected reductive group over $\mathrm{F}$. An endoscopic triple for $\mathrm{G}$ is a triple $(\mathrm{H}, s, \eta)$, where $\mathrm{H}$ is a quasi-split connected reductive group over $\mathrm{F}, s$ is an element of $Z(\hat{\mathrm{H}})$ and $\eta: \hat{\mathrm{H}} \rightarrow \hat{\mathrm{G}}$ is an embedding of complex Lie groups. The triple has to satisfy certain conditions, as in 7.4 of $[\operatorname{Kot} 84$. Let $\Gamma:=\operatorname{Gal}(\overline{\mathbb{Q}} / \mathbb{Q})$. An endoscopic triple is called elliptic if $\left(Z(\hat{\mathrm{H}})^{\Gamma}\right)^{\circ} \subset Z(\mathrm{G})$. We will use the notion of isomorphism of endoscopic triples in Section 2.1 of Shi10, which is stronger than the one in Kot84. We let $\mathcal{E}^{\text {ell }}(\mathrm{G})$ be the set of isomorphism classes of elliptic endoscopic triples for $\mathrm{G}$.

Assume that $\mathrm{G}^{\mathrm{der}}$ is simply-connected (this will be the case for $\mathrm{G}:=G$, our unitary similitude group). We use Weil groups to construct $L$-groups; then we can choose an extension of $\eta$ to an $L$-group morphism $\tilde{\eta}:{ }^{L} \mathrm{H} \rightarrow{ }^{L} \mathrm{G}$ by Proposition 1 of $\operatorname{Lan} 79$.

Assume that F is a local field. Given $\tilde{\eta}$, Langlands and Shelstad (see [LS87]) define a transfer factor

$$
\Delta: \mathrm{H}(\mathrm{F})_{\mathrm{ss},(\mathrm{G}, \mathrm{H})-\mathrm{reg}} \times \mathrm{G}(\mathrm{F})_{\mathrm{ss}} \rightarrow \mathbb{C},
$$

which is canonical up to a non-zero constant.

The fundamental lemma and the transfer conjecture, which are now theorems due to Ngo, Waldspurger and others (see [Ngô10, Wal97]), assert that for each function $\phi \in C_{c}^{\infty}(\mathrm{G}(\mathrm{F}))$, there exists $\phi^{\mathrm{H}} \in C_{c}^{\infty}(\mathrm{H}(\mathrm{F}))$ satisfying an identity about the transfer of orbital integrals

$$
S O_{\gamma_{\mathrm{H}}}^{\mathrm{H}(\mathrm{F})}\left(\phi^{\mathrm{H}}\right)=\sum_{\gamma \in \mathrm{G}(\mathrm{F})_{\mathrm{ss}} / \sim} \Delta\left(\gamma_{\mathrm{H}}, \gamma\right) e\left(\mathrm{G}_{\gamma}\right) O_{\gamma}^{\mathrm{G}(\mathrm{F})}(\phi)
$$

(see Theorem 3.1 of [SS13] for an explanation of the notation). If H, G and $\tilde{\eta}$ are unramified and if $\phi \in \mathcal{H}^{\mathrm{ur}}(\mathrm{G}(\mathrm{F}))$, then $\Delta$ can be normalized such that $\phi^{\mathrm{H}}$ can be taken to be $\tilde{\eta}^{*}(\phi)$, where $\tilde{\eta}^{*}: \mathcal{H}^{\mathrm{ur}}(\mathrm{G}(\mathrm{F})) \rightarrow \mathcal{H}^{\mathrm{ur}}(\mathrm{H}(\mathrm{F}))$ is the morphism of unramified Hecke algebras induced from $\tilde{\eta}$ via the Satake isomorphism. In particular, if 
$\phi$ is the idempotent associated to a hyperspecial maximal compact subgroup, then $\phi^{\mathrm{H}}$ can also be taken to be the idempotent of a hyperspecial maximal compact subgroup.

Let $\phi \in C_{c}^{\infty}\left(G\left(\mathbb{A}_{f}^{p}\right) \times J_{b}\left(\mathbb{Q}_{p}\right)\right)$ be an acceptable function of the form

$$
\phi=\prod_{v \neq \infty} \phi_{v}, \text { with } \phi_{v} \in C_{c}^{\infty}\left(G\left(\mathbb{Q}_{v}\right)\right), v \neq p, \phi_{p} \in C_{c}^{\infty}\left(J_{b}\left(\mathbb{Q}_{p}\right)\right) .
$$

Let $(H, s, \eta) \in \mathcal{E}^{\mathrm{ell}}(G)$.

Definition 5.2.2. Let $\phi^{H}:=\phi^{H, p} \phi_{p}^{H} \phi_{\infty}^{H} \in C_{c}^{\infty}(H(\mathbb{A}))$, where:

- $\phi^{H, p}$ is the Langlands-Shelstad transfer of $\phi^{p}$ (as described above);

- $\phi_{\infty}^{H}$ is constructed by Kottwitz in Section 7 of Kot90, where we take the trivial algebraic representation of $G$ as an input (this corresponds to the fact that our local system on $\mathscr{I}_{\text {Mant }}^{b}$ is $\overline{\mathbb{Q}}_{\ell}$.) We give more details in the case when $G$ is a unitary similitude group below.

- $\phi_{p}^{H}$ is constructed in Section 6 of [Shi10]. The function $\phi_{p}^{H}$ is the key construction of [Shi10]; we give more details in Section 5.4 below.

The following is the main result of [Shi10], Theorem 7.2 of loc. cit.

Theorem 5.2.3. Let $\phi$ and $\phi^{H}$ be as above, with $(H, s, \eta) \in \mathcal{E}^{\mathrm{ell}}(G)$. Then

$$
\operatorname{tr}\left(\phi \mid \iota_{\ell} H_{c}\left(\mathscr{I}_{\text {Mant }}^{b}, \overline{\mathbb{Q}}_{\ell}\right)\right)=\left|\operatorname{ker}^{1}(\mathbb{Q}, G)\right| \sum_{(H, s, \eta)} \iota(G, H) S T_{e}^{H}\left(\phi^{H}\right) .
$$

Remark 5.2.4. Shin's result is in fact valid for any PEL Shimura variety of type $(A)$ or $(C)$. We recall that

$$
\operatorname{ker}^{1}(\mathbb{Q}, G):=\operatorname{ker}\left(H^{1}(\mathbb{Q}, G) \rightarrow \prod_{v} H^{1}\left(\mathbb{Q}_{v}, G\right)\right),
$$

and that $S_{K}$ is the disjoint union of $\left|\operatorname{ker}^{1}(\mathbb{Q}, G)\right|$ copies of the Shimura variety for $G$. Also, $\iota(G, H):=\tau(G) \tau(H)^{-1}|\operatorname{Out}(H, s, \eta)|^{-1}$. The term $S T_{e}^{H}\left(\phi^{H}\right)$ is a sum of stable orbital integrals over (representatives of) $\mathbb{Q}$-elliptic semisimple stable conjugacy classes in $H(\mathbb{Q})$.

In the case of our unitary similitude group $G$, the set $\mathcal{E}^{\text {ell }}(G)$ only depends on the quasi-split inner form $G_{n}$ of $G$ and in [Shi11, Shin gives a concrete description of a set of representatives for the isomorphism classes in $\mathcal{E}^{\mathrm{ell}}\left(G_{n}\right)$. If $\vec{n}=\left(n_{i}\right)_{i=1}^{s}$ is a vector with entries positive integers, one can define a quasi-split group $G_{\vec{n}}$ over $\mathbb{Q}$ as in Section 3.1 of [Shi11. Define $G L_{\vec{n}}:=\prod_{i=1}^{s} G L_{n_{i}}$ and let $i_{\vec{n}}: G L_{\vec{n}} \rightarrow G L_{\left(\sum_{i} n_{i}\right)}$ be the natural map. Let

$$
\Phi_{\vec{n}}:=i_{\vec{n}}\left(\Phi_{n_{1}}, \ldots, \Phi_{n_{s}}\right),
$$

where $\Phi_{n}$ is the matrix in $G L_{n}$ with entries $\left(\Phi_{n}\right)_{i j}=(-1)^{i+1} \delta_{i, n+1-j}$. Then $G_{\vec{n}}$ is the algebraic group over $\mathbb{Q}$ sending a $\mathbb{Q}$-algebra $R$ to

$$
G_{\vec{n}}(R)=\left\{(\lambda, g) \in R^{\times} \times G L_{\vec{n}}\left(F \otimes_{\mathbb{Q}} R\right) \mid g \cdot \Phi_{\vec{n}} \cdot{ }^{t} g^{c}=\lambda \Phi_{\vec{n}}\right\} .
$$

Since $G$ is quasi-split at all finite places, we have

$$
G \times \mathbb{Q} \mathbb{A}_{f} \simeq G_{n} \times_{\mathbb{Q}} \mathbb{A}_{f}
$$

and we fix such an isomorphism. 
The representatives for $\mathcal{E}^{\text {ell }}\left(G_{n}\right)$ can be taken to be

$$
\left\{\left(G_{n}, s_{n}, \eta_{n}\right)\right\} \cup\left\{\left(G_{n_{1}, n_{2}}, s_{n_{1}, n_{2}}, \eta_{n_{1}, n_{2}}\right) \mid n_{1}+n_{2}=n, n_{1} \geq n_{2} \geq 0\right\},
$$

where $\left(n_{1}, n_{2}\right)$ may be excluded if both $n_{1}$ and $n_{2}$ are odd numbers (see condition 7.4.3 of [Kot84]). Here, $s_{n}=1 \in \hat{G}_{n}, s_{n_{1}, n_{2}}=\left(1,\left(I_{n_{1}},-I_{n_{2}}\right)\right) \in \hat{G}_{n_{1}, n_{2}}, \eta_{n}$ is the identity map and $\eta_{n_{1}, n_{2}}: \hat{G}_{n_{1}, n_{2}} \rightarrow \hat{G}_{n}$ is the natural embedding induced by $G L_{n_{1}} \times G L_{n_{2}} \hookrightarrow G L_{n}$.

If we choose a Hecke character $\psi: \mathbb{A}_{\mathcal{K}}^{\times} / \mathcal{K}^{\times} \rightarrow \mathbb{C}^{\times}$such that $\left.\psi\right|_{\mathbb{A}^{\times} / \mathbb{Q}^{\times}}$corresponds via class field theory to the quadratic character associated to $\mathcal{K} / \mathbb{Q}$, we can extend $\eta_{n_{1}, n_{2}}$ to an $L$-morphism

$$
\tilde{\eta}_{n_{1}, n_{2}}:{ }^{L} G_{n_{1}, n_{2}} \rightarrow{ }^{L} G_{n}
$$

(See Section 3.2 of Shi11 for the precise formula.) By Proposition 7.1 of Shi11, $\psi$ can be chosen such that the set of primes where $\psi$ is ramified is contained in $\mathrm{Spl}_{F / F^{+}}$. As a consequence, we can use the explicit transfer factors described in Section 3.4 of [Shi1] at all places not equal to $p, \infty$. These are compatible with the Langlands-Shelstad transfer described above: at unramified places $v$, we take

$$
\tilde{\eta}_{n_{1}, n_{2}}^{*}: \mathcal{H}^{\mathrm{ur}}\left(G_{n}\left(\mathbb{Q}_{v}\right)\right) \rightarrow \mathcal{H}^{\mathrm{ur}}\left(G_{n_{1}, n_{2}}\left(\mathbb{Q}_{v}\right)\right),
$$

making use of the fundamental lemma [Nô10]. Since we have fixed an isomorphism $G \times \mathbb{Q} \mathbb{A}_{f} \stackrel{\sim}{\rightarrow} G_{n} \times \mathbb{Q} \mathbb{A}_{f}$, we can also think of this as a transfer from $G$ to $G_{n_{1}, n_{2}}$ at places away from $p, \infty$.

We also describe the explicit transfer at the place $\infty$. The transfer is as in Section 7 of Kot90 and uses Shelstad's theory of real endoscopy and the Langlands correspondence for real reductive groups; see also Section 3.5 of [Shi1] for any unfamiliar notation. Recall that over $\mathbb{R}, G$ is an inner form of the quasi-split unitary similitude group $G_{n}$. For any discrete $L$-parameter $\varphi_{G_{\vec{n}}}$ for $G_{\vec{n}}$, with $L$ packet $\Pi\left(\varphi_{G_{\vec{n}}}\right)$, define

$$
\phi_{\varphi_{\vec{n}}}:=\frac{1}{\left|\Pi\left(\varphi_{G_{\vec{n}}}\right)\right|} \sum_{\pi \in \Pi\left(\varphi_{G_{\vec{n}}}\right)} \phi_{\pi}
$$

where $\phi_{\pi}$ is a pseudo-coefficient for $\pi$. When $\varphi_{G_{\vec{n}}} \sim \varphi_{\xi}$ corresponds to an $L$ packet $\Pi_{\text {disc }}\left(G_{\vec{n}}(\mathbb{R}), \xi^{\vee}\right)$ for some irreducible algebraic representation $\xi$ of $G_{\vec{n}}$, the function $\phi_{\varphi_{G_{\vec{n}}}}$ is called an Euler-Poincaré function; we denote it also by $\phi_{G_{\vec{n}}, \xi}$. The desired function $\phi_{\infty}^{\vec{n}}$ will be a precise linear combination of the Euler-Poincaré functions for $L$-parameters $\varphi_{G_{\vec{n}}}$ for which $\tilde{\eta} \circ \varphi_{G_{\vec{n}}}$ corresponds to the trivial algebraic representation of $G_{\mathbb{C}}$ (see 5.11 of [Shi11] for the precise formula).

For further use, we record a version of Theorem 5.2 .3 for the group $G$.

Corollary 5.2.5. If $\phi^{p} \cdot \phi_{p} \in C_{c}^{\infty}\left(G\left(\mathbb{A}_{f}^{p}\right) \times J_{b}\left(\mathbb{Q}_{p}\right)\right)$ is acceptable, then

$$
\operatorname{tr}\left(\phi \mid \iota_{\ell} H_{c}\left(\mathscr{I}_{\text {Mant }}^{b}, \overline{\mathbb{Q}}_{\ell}\right)\right)=\left|\operatorname{ker}^{1}(\mathbb{Q}, G)\right| \sum_{G_{\vec{n}}} \iota\left(G, G_{\vec{n}}\right) S T_{e}^{G_{\vec{n}}}\left(\phi^{\vec{n}}\right),
$$

where $G_{\vec{n}}$ runs over the set described above and $\phi^{\vec{n}}$ is obtained from $\phi$ as in Definition 5.2.2.

Remark 5.2.6. The constants $\iota\left(G, G_{\vec{n}}\right)$ can be computed explicitly:

$$
\iota\left(G, G_{\vec{n}}\right)= \begin{cases}\frac{1}{2} \tau(G) \tau\left(G_{\vec{n}}\right)^{-1} & \text { if } \vec{n}=\left(\frac{n}{2}, \frac{n}{2}\right) \\ \tau(G) \tau\left(G_{\vec{n}}\right)^{-1} & \text { otherwise }\end{cases}
$$


5.3. Base change and the twisted trace formula. Let $\mathbb{G}_{\vec{n}}:=\operatorname{Res}_{\mathcal{K} / \mathbb{Q}}\left(G_{\vec{n}} \times_{\mathbb{Q}} \mathcal{K}\right)$. One can define $L$-morphisms $B C_{\vec{n}}:{ }^{L} G_{\vec{n}} \rightarrow{ }^{L} \mathbb{G}_{\vec{n}}$ and $\tilde{\zeta}_{n_{1}, n_{2}}:{ }^{L} \mathbb{G}_{n_{1}, n_{2}} \rightarrow{ }^{L} \mathbb{G}_{n}$ and there is a commutative diagram of $L$-morphisms

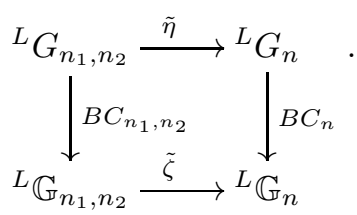

In this section, we review the associated base change for the groups $G_{\vec{n}}$ and $\mathbb{G}_{\vec{n}}$ as well as the twisted trace formula. Let $S$ be a finite set of primes containing $\infty, p$ and all the primes where either the CM field $F$ or the Hecke character $\psi$ are ramified. Recall that, by the assumptions in Section 5.1. we can and will arrange that $S_{\text {fin }} \subset \operatorname{Spl}_{F / F^{+}}$.

We can define a notion of $B C$-transfer of functions as in Section 4 of Shi11. If $v$ is a finite place of $\mathbb{Q}$ such that $v \notin S$, then the dual map to the $L$-morphism $B C_{\vec{n}}$ defines the transfer

$$
B C_{\vec{n}}^{*}: \mathcal{H}^{\mathrm{ur}}\left(\mathbb{G}_{\vec{n}}\left(\mathbb{Q}_{v}\right)\right) \rightarrow \mathcal{H}^{\mathrm{ur}}\left(G_{\vec{n}}\left(\mathbb{Q}_{v}\right)\right),
$$

(Case 1) of Section 4.2 of Shi11. Otherwise, if $v \in S_{\mathrm{fin}} \subset \operatorname{Spl}_{F / F^{+}}$then Section 4.2 of loc. cit., (Case 2), constructs a $B C$-transfer $\phi_{v} \in C_{c}^{\infty}\left(G_{\vec{n}}\left(\mathbb{Q}_{v}\right)\right)$ of $f_{v} \in$ $C_{c}^{\infty}\left(\mathbb{G}_{\vec{n}}\left(\mathbb{Q}_{v}\right)\right)$. We remark that, if $v$ splits in $\mathcal{K}$ (e.g. if $v=p$ ), one can check directly that $B C_{\vec{n}}^{*}$ is surjective. It is also possible to define a transfer $\tilde{\zeta}_{\vec{n}}^{*}$, as in Section 4 of loc. cit., making the obvious diagram commutative.

At $\infty$, the transfer is defined in Section 4.3 of loc. cit. Let $\xi$ be an irreducible algebraic representation of $\left(G_{\vec{n}}\right)_{\mathbb{C}}$, giving rise to the representation $\Xi$ of $\left(\mathbb{G}_{\vec{n}}\right)_{\mathbb{C}}$ which is just $\Xi:=\xi \otimes \xi$. Recall that $\phi_{G_{\vec{n}}, \xi}$ is the Euler-Poincaré function for $\xi$. Associated to $\Xi$, Labesse defined a twisted analogue of the Euler-Poincaré function, a Lefschetz

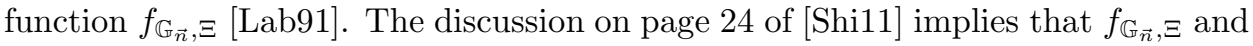
$\phi_{G_{\vec{n}}, \xi}$ are $B C$-matching functions.

Define the group

$$
\mathbb{G}_{\vec{n}}^{+}:=\left(\operatorname{Res}_{\mathcal{K} / \mathbb{Q}} G L_{1} \times \operatorname{Res}_{F / \mathbb{Q}} G L_{\vec{n}}\right) \rtimes\{1, \theta\},
$$

where $\theta(\lambda, g) \theta^{-1}=\left(\lambda^{c}, \lambda^{c} g^{\sharp}\right)$ and $g^{\sharp}=\Phi_{\vec{n}}^{t} g^{c} \Phi_{\vec{n}}^{-1}$. If we denote by $\mathbb{G}_{\vec{n}}^{\circ}$ and $\mathbb{G}_{\vec{n}}^{\circ} \theta$ the cosets of $\{1\}$ and $\{\theta\}$ in $\mathbb{G}_{\vec{n}}^{+}$, then $\mathbb{G}_{\vec{n}}^{+}=\mathbb{G}_{\vec{n}}^{\circ} \sqcup \mathbb{G}_{\vec{n}}^{\circ} \theta$. There is a natural $\mathbb{Q}$ isomorphism $\mathbb{G}_{\vec{n}} \stackrel{\sim}{\rightarrow} \mathbb{G}_{\vec{n}}^{\circ}$, which extends to an isomorphism

$$
\mathbb{G}_{\vec{n}} \rtimes \operatorname{Gal}(\mathcal{K} / \mathbb{Q}) \stackrel{\sim}{\rightarrow} \mathbb{G}_{\vec{n}}^{+}
$$

so that $c \in \operatorname{Gal}(\mathcal{K} / \mathbb{Q})$ maps to $\theta$. Using this isomorphism, we write $\mathbb{G}_{\vec{n}}$ and $\mathbb{G}_{\vec{n}} \theta$ for the two cosets.

If $f \in C_{c}^{\infty}\left(\mathbb{G}_{\vec{n}}(\mathbb{A})\right.$ ) (with trivial character on $A_{\mathbb{G}_{n}, \infty}^{\circ}$ ), then we define $f \theta$ to be the function on $\mathbb{G}_{\vec{n}} \theta(\mathbb{A})$ obtained via translation by $\theta$. The (invariant) twisted trace formula (see [Art88a, Art88b) gives an equality

$$
I_{\text {geom }}^{\mathbb{G} \vec{n} \theta}(f \theta)=I_{\text {spec }}^{\mathbb{G} \vec{n}} \theta(f \theta) .
$$

The left hand side of the equation is defined in Section 3 of Art88b, while the right hand side is defined in Section 4 of loc. cit.

Let $f_{\mathbb{G}_{\vec{n}}, \Xi}$ and $\phi_{G_{\vec{n}}, \xi}$ be as defined above. The following is Corollary 4.7 of [Shi11]. 
Proposition 5.3.1. We have the following equality:

$$
I_{\text {geom }}^{\mathbb{G} \vec{n} \theta}(f \theta)=\tau\left(G_{\vec{n}}\right)^{-1} S T_{e}^{G_{\vec{n}}}(\phi),
$$

when $\phi$ and $f$ satisfy

$$
\phi=\phi^{S} \cdot \phi_{S_{\mathrm{fin}}} \cdot \phi_{G_{\vec{n}}, \xi} \text { and } f=f^{S} \cdot f_{S_{\mathrm{fin}}} \cdot f_{\mathbb{G}_{\vec{n}}, \Xi}
$$

with $\phi^{S}$ a BC-transfer of $f^{S}, \phi_{S_{\mathrm{fin}}}$ a BC-transfer of $f_{S_{\mathrm{fin}}}$.

Proof. We sketch the proof here: first, use Theorem 4.3.4 of [Lab99] to rewrite the sum of stable orbital integrals on the right as the elliptic part of the twisted trace formula for $\mathbb{G}_{\vec{n}} \theta$. Then the geometric side of the twisted trace formula for $\mathbb{G}_{\vec{n}} \theta$ is simplified using similar techniques to those in Chapter 7 of Art88b]: the key facts are that the Lefschetz function $f_{\mathbb{G}_{\vec{n}}} \Xi$ is cuspidal, so only $\theta$-elliptic elements contribute, and that $\left[F^{+}: \mathbb{Q}\right] \geq 2$, so that the only Levi subgroup that contributes to the geometric side is $\mathbb{G}_{\vec{n}} \theta$ itself.

We now explain how to construct our test functions, which is exactly as in the proof of Theorem 6.1 of [Shi11]. We let $\left(f^{n}\right)^{S}$ be any function in $\mathcal{H}^{\mathrm{ur}}\left(\mathbb{G}_{n}\left(\mathbb{A}^{S}\right)\right)$ and $f_{S_{\mathrm{fin}} \backslash\{p\}}^{n}$ be any function in $C_{c}^{\infty}\left(\mathbb{G}_{n}\left(\mathbb{A}_{S_{\mathrm{fin} \backslash\{p\}}}\right)\right)$. We let $\phi^{S}, \phi_{S_{\mathrm{fin} \backslash\{p\}}}$ be their $B C$-transfers, as described above. We take $\phi_{p} \in C_{c}^{\infty}\left(J_{b}\left(\mathbb{Q}_{p}\right)\right)$ be any acceptable function and set

$$
\phi:=\phi^{S} \cdot \phi_{S_{\mathrm{fin} \backslash\{p\}}} \cdot \phi_{p} .
$$

From these test functions, we construct all the other test functions we will need. First, for each elliptic endoscopic group $G_{\vec{n}}$ we let $\phi^{\vec{n}}$ be constructed from $\phi$ as in Definition 5.2.2. Let $\left(f^{n_{1}, n_{2}}\right)^{S}$ and $\left(f^{n_{1}, n_{2}}\right)_{S_{\text {fin } \backslash\{p\}}}$ be obtained from $\left(f^{n}\right)^{S}$ and $f_{S_{\text {fin } \backslash\{p\}}}^{n}$ by transfer along the $L$-morphism $\tilde{\zeta}$. We choose $f_{p}^{\vec{n}}$ so that $B C_{\vec{n}}^{*}\left(f_{p}^{\vec{n}}\right)=$ $\phi_{p}^{\vec{n}}$ (recall that $B C_{\vec{n}}^{*}$ is surjective at $p$ ). We can define $f_{\infty}^{\vec{n}}$ explicitly, as a linear combination of Lefschetz functions for representations $\Xi\left(\varphi_{\vec{n}}\right)$ of $\mathbb{G}_{\vec{n}}$ for which $\tilde{\eta} \circ \varphi_{\vec{n}}$ corresponds to the trivial representation of $G$ (see (6.7) of [Shi11] for the precise formula). Finally, we set

$$
f^{\vec{n}}:=\left(f^{\vec{n}}\right)^{S} \cdot\left(f^{\vec{n}}\right)_{S_{\mathrm{fin} \backslash\{p\}}} \cdot f_{p}^{\vec{n}} \cdot f_{\infty}^{\vec{n}} .
$$

By the commutative diagram of $L$-morphisms (5.3.1), we can apply Proposition 5.3.1 to $f^{\vec{n}}$ and $\phi^{\vec{n}}$. To check the compatibility, see (4.18) of [Shi11] for primes away from $S$, (4.19) of loc. cit. for primes in $S_{\text {fin }} \backslash\{p\}$ and compare the precise formulas for $\phi_{\infty}^{\vec{n}}$ and $f_{\infty}^{\vec{n}}$. We mention that the formulas for $\phi_{\infty}^{\vec{n}}$ and $f_{\infty}^{\vec{n}}$ use as input an inner form $G$ of $G_{n}$ over $\mathbb{R}$; in loc. cit. this inner form has a specific signature (a group of so-called Harris-Taylor type), but here we work more generally. In particular, the integer $q(G)$ appearing there is defined as $\frac{1}{2} \operatorname{dim}\left(G(\mathbb{R}) / K_{\infty} A_{\infty}^{\circ}\right)$.

Theorem 5.3.2. We have an equality

$$
\operatorname{tr}\left(\phi \mid \iota_{\ell}\left[H_{c}\left(\mathscr{I}_{\text {Mant }}^{b}, \overline{\mathbb{Q}}_{\ell}\right)\right]\right)=\left|\operatorname{ker}^{1}(\mathbb{Q}, G)\right| \tau(G) \sum_{G_{\vec{n}}} \epsilon_{\vec{n}} \cdot I_{\text {spec }}^{\mathbb{G}_{\vec{n}} \theta}\left(f^{\vec{n}} \theta\right)
$$

where $\epsilon_{\vec{n}}=\frac{1}{2}$ if $\vec{n}=\left(\frac{n}{2}, \frac{n}{2}\right)$ and $\epsilon_{\vec{n}}=1$ otherwise.

Proof. This follows by combining Corollary 5.2.5, Remark 5.2.6 Proposition 5.3.1 and equation (5.3.2). 
Fix $G_{\vec{n}}$. We now proceed to simplify the spectral side $I_{\text {spec }}^{\mathbb{G}_{\vec{n}} \theta}\left(f^{\vec{n}} \theta\right)$. We need the following notation from [Shi1]: let $M_{0}$ be a minimal Levi subgroup of $\mathbb{G}_{\vec{n}}$. For $M$ a rational Levi of $\mathbb{G}_{\vec{n}}$ containing $M_{0}$, choose a parabolic subgroup $Q$ containing $M$ as a Levi. The group $W^{\mathbb{G}_{\vec{n}} \theta}\left(\mathfrak{a}_{M}\right)_{\text {reg }}$ defined in Art88b] acts on the set of parabolic subgroups which have $M$ as a Levi component. The automorphism $\Phi_{\vec{n}}^{-1} \theta$ of $\mathbb{G}_{\vec{n}}$ preserves $M$ and acts on $W^{\mathbb{G}_{\vec{n}} \theta}\left(\mathfrak{a}_{M}\right)_{\text {reg. }}$. By combining Proposition 4.8 and Corollary 4.14 of Shi11, we have the following expression for the summands on the right hand side of Theorem 5.3 .2 .

Proposition 5.3.3. There is an equality

$$
I_{\text {spec }}^{\mathbb{G}_{\vec{n}} \theta}\left(f^{\vec{n}} \theta\right)=\sum_{M} \frac{\left|W_{M}\right|}{\left|W_{G}\right|}\left|\operatorname{det}\left(\Phi_{\vec{n}}^{-1} \theta-1\right)_{\mathfrak{a}_{M}^{\mathbb{G}_{\vec{n}}} \theta}\right|^{-1} \sum_{\Pi_{M}} \operatorname{tr}\left(\mathrm{n}-\operatorname{Ind}_{Q}^{\mathbb{G}_{\vec{n}}}\left(\Pi_{M}\right)_{\xi}\left(f^{\vec{n}}\right) \circ A^{\prime}\right)
$$

where $M$ runs over the Levi subgroups of $\mathbb{G}_{\vec{n}}$ containing $M_{0}$ and $\Pi_{M}$ runs over the irreducible $\Phi_{\vec{n}}^{-1} \theta$-stable subrepresentations of the discrete spectrum $R_{M \text {,disc }}$.

Remark 5.3.4. The subscript $\xi$ indicates a possible twist by a character of $A_{\mathbb{G}_{\vec{n}}, \infty}^{\circ}$ corresponding to an irreducible algebraic representation $\xi$ of $G_{\vec{n}}$ and $A^{\prime}$ is a normalized intertwiner on $\mathrm{n}-\operatorname{Ind}_{Q}^{\mathbb{G}_{\vec{n}}}\left(\Pi_{M}\right)_{\xi}$. We do not make this precise, as we will not need these details. We do note that, as $\Pi_{M}$ is $\Phi_{\vec{n}}^{-1} \theta$-stable, $\mathrm{n}-\operatorname{Ind}_{Q}^{\mathbb{G}_{\vec{n}}}\left(\Pi_{M}\right)_{\xi}$ is $\theta$-stable.

5.4. The transfer at $p$. We recall the construction of the function $\phi_{p}^{\vec{n}}$, starting from an acceptable function $\phi_{p} \in C_{c}^{\infty}\left(J_{b}\left(\mathbb{Q}_{p}\right)\right)$, as well as the representationtheoretic counterpart to this construction, $\operatorname{Red}_{\vec{n}}^{b}$.

The group $J_{b}\left(\mathbb{Q}_{p}\right)$ is an inner form of a Levi subgroup $M_{b}\left(\mathbb{Q}_{p}\right)$ of $G\left(\mathbb{Q}_{p}\right)$; for further reference, we recall their precise definitions, following Chapter 1 of [RZ96]. According to Definition 1.8 of loc. cit., an element $\tilde{b}$ of $G(L)$ is called decent if there exists a positive integer $s$ such that

$$
(\tilde{b} \sigma)^{s}=s \nu_{\tilde{b}}(p) \sigma^{s}
$$

where $s \nu_{\tilde{b}}$ factors through a morphism $\mathbb{G}_{m} \rightarrow G$. By Section 4.3 of [Kot85], any $\sigma$-conjugacy class $b \in B(G)$ admits a decent representative $\tilde{b}$; as $G$ is quasisplit, one can moreover arrange that $\nu_{\tilde{b}}$ is defined over $\mathbb{Q}_{p}$, cf. [Kot85, p. 219]. Let $M_{b}$ be the centralizer of $\nu$ in $G$, which is a $\mathbb{Q}_{p}$-rational Levi subgroup. Then $b$ is a basic element of $M_{b}$, and $J_{b}$ is an inner form of $M_{b}$.

Fix $G_{\vec{n}}$ an elliptic endoscopic group for $G$. The set $\mathcal{E}_{p}^{\text {eff }}\left(J_{b}, G, G_{\vec{n}}\right)$ is defined in Section 6.2 of [Shi09]; it consists of certain isomorphism classes $\left(M_{G_{\vec{n}}}, s_{\vec{n}}, \eta_{\vec{n}}\right)$ of $G_{\vec{n}}$-endoscopic triples for $J_{b}\left(\mathbb{Q}_{p}\right)$. The function $\phi_{p}^{\vec{n}}$ is constructed via transfer from $\phi_{p}$ on $J_{b}\left(\mathbb{Q}_{p}\right)$ to $M_{G_{\vec{n}}}\left(\mathbb{Q}_{p}\right)$, followed by a version of transfer from $M_{G_{\vec{n}}}\left(\mathbb{Q}_{p}\right)$ to $G_{\vec{n}}\left(\mathbb{Q}_{p}\right)$. We remark that the latter step makes crucial use of the acceptability of $\phi_{p}$, cf. Lemma 3.9 of [Shi10].

There is a representation-theoretic counterpart to this construction. This is a group morphism

$$
\operatorname{Red}_{\vec{n}}^{b}: \operatorname{Groth}\left(G_{\vec{n}}\left(\mathbb{Q}_{p}\right)\right) \rightarrow \operatorname{Groth}\left(J_{b}\left(\mathbb{Q}_{p}\right)\right)
$$

$\operatorname{Red}_{\vec{n}}^{b}$ will be defined as the composition of the following maps:

(1)

$$
\operatorname{Groth}\left(G_{\vec{n}}\left(\mathbb{Q}_{p}\right)\right) \rightarrow \bigoplus_{\left(M_{G_{\vec{n}}}, s_{\vec{n}}, \eta_{\vec{n}}\right)} \operatorname{Groth}\left(M_{G_{\vec{n}}}\left(\mathbb{Q}_{p}\right)\right)
$$


where the sum runs over $G_{\vec{n}}$-endoscopic triples in $\mathcal{E}_{p}^{\text {eff }}\left(J_{b}, G, G_{\vec{n}}\right)$ and the map on each term is a linear combination of normalized Jacquet functors (2) (indexed over a finite set of allowed Levi embeddings $M_{G_{\vec{n}}} \hookrightarrow G_{\vec{n}}$ );

$$
\operatorname{Groth}\left(M_{G_{\vec{n}}}\left(\mathbb{Q}_{p}\right)\right) \rightarrow \operatorname{Groth}\left(M_{b}\left(\mathbb{Q}_{p}\right)\right)
$$

which is the functorial transfer with respect to the $L$-morphism $\tilde{\eta}_{\vec{n}}$. Both $M_{G_{\vec{n}}}$ and $M_{b}$ are (restrictions of scalars of) products of general linear groups and the transfer ends up being a normalized parabolic induction.

$$
\operatorname{Groth}\left(M_{b}\left(\mathbb{Q}_{p}\right)\right) \rightarrow \operatorname{Groth}\left(J_{b}\left(\mathbb{Q}_{p}\right)\right),
$$

which is the Langlands-Jacquet map on Grothendieck groups, defined by Bad07.

(See Section 5.5 of Shi11 for the precise definition of these three maps; even though the case we are considering is slightly more general, the formulas will be exact analogues.)

Remark 5.4.1. When $\vec{n}=(n), \mathcal{E}_{p}^{\text {eff }}\left(J_{b}, G, G_{\vec{n}}\right)$ has only one element, namely $\left(M_{b}, 1\right.$, id). The morphism $\operatorname{Red}_{n}^{b}$ consists of a normalized Jacquet functor followed by the Langlands-Jacquet map.

We record the relationship between $\operatorname{Red}_{\vec{n}}^{b}$ and $\phi_{p}^{\vec{n}}$ in the following lemma.

Lemma 5.4.2. For any $\pi_{p} \in \operatorname{Groth}\left(G_{\vec{n}}\left(\mathbb{Q}_{p}\right)\right)$,

$$
\operatorname{tr} \pi_{p}\left(\phi_{p}^{\vec{n}}\right)=\operatorname{tr}\left(\operatorname{Red}_{\vec{n}}^{b}\left(\pi_{p}\right)\right)\left(\phi_{p}\right) .
$$

Proof. The statement follows in the same way as Lemma 5.10 of Shi11 (see also Lemmas 6.3 and 6.4 of Car12 for a unitary group with a slightly different signature). The idea is that the constructions of both $\operatorname{Red}_{\vec{n}}^{b}$ and $\phi^{\vec{n}}$ can be broken down into the three steps outlined above and the constructions in each of these steps are dual to each other. One of the key points is that the transfer of $\phi_{p}$ from $J_{b}\left(\mathbb{Q}_{p}\right)$ to $M_{G_{\vec{n}}}\left(\mathbb{Q}_{p}\right)$ can be broken down into transfer from $J_{b}\left(\mathbb{Q}_{p}\right)$ to the quasisplit form $M_{b}\left(\mathbb{Q}_{p}\right)$ followed by transfer from $M_{b}\left(\mathbb{Q}_{p}\right)$ to $M_{G_{\vec{n}}}\left(\mathbb{Q}_{p}\right)$. The other key point is the slightly non-standard transfer between $M_{G_{\vec{n}}}$ and $G_{\vec{n}}$, where the desired compatibility follows from Lemma 3.9 of Shi10.

We note that the whole situation decomposes into a product. Namely, let $\mathfrak{p}_{1}, \ldots, \mathfrak{p}_{m}$ be the primes of $F^{+}$above $p$, and fix a decomposition $p=u u^{c}$ in $\mathcal{K}$. We denote by $\mathfrak{p}_{i}$ also the place of $F$ lying over $\mathfrak{p}_{i}$ in $F^{+}$, and $u$ in $\mathcal{K}$, and by $\mathfrak{p}_{i}^{c}$ the complex conjugate place of $F$. With these choices, we get a decomposition

$$
G_{\mathbb{Q}_{p}}=\prod_{i} \operatorname{Res}_{F_{\mathfrak{p}_{i}} / \mathbb{Q}_{p}} G L_{n} \times \mathbb{G}_{m}
$$

Here, the projection to the $\mathbb{G}_{m}$-factor is the unitary similitude factor, and the projection to the general linear groups is via the projection

$$
V \otimes_{\mathbb{Q}} \mathbb{Q}_{p}=\bigoplus_{i}\left(V \otimes_{F} F_{\mathfrak{p}_{i}} \oplus V \otimes_{F} F_{\mathfrak{p}_{i}^{c}}\right) \rightarrow \bigoplus_{i} V \otimes_{F} F_{\mathfrak{p}_{i}}
$$

The resulting constructions above admit similar decompositions. In particular,

$$
b=\left(\left(b_{i}\right)_{i=1, \ldots, m}, b_{0}\right) \in B(G)=\prod_{i=1}^{m} B\left(\operatorname{Res}_{F_{\mathfrak{p}_{i}} / \mathbb{Q}_{p}} G L_{n}\right) \times B\left(\mathbb{G}_{m}\right),
$$


and $J_{b}=\prod_{i=1}^{m} J_{b_{i}} \times \mathbb{G}_{m}$. Also, any irreducible representation $\pi_{p}$ of $G\left(\mathbb{Q}_{p}\right)$ decomposes into a tensor product

$$
\pi_{p}=\bigotimes_{i=1}^{m} \pi_{\mathfrak{p}_{i}} \otimes \pi_{0}
$$

where $\pi_{\mathfrak{p}_{i}}$ is an irreducible representation of $G L_{n}\left(F_{\mathfrak{p}_{i}}\right)$, and $\pi_{0}$ is a character of $\mathbb{Q}_{p}^{\times}$. A similar discussion applies to representations of

$$
G_{\vec{n}}\left(\mathbb{Q}_{p}\right)=\prod_{i=1}^{m} G L_{\vec{n}}\left(F_{\mathfrak{p}_{i}}\right) \times \mathbb{Q}_{p}^{\times} .
$$

Lemma 5.4.3. Let $\pi_{p}^{\vec{n}} \in \operatorname{Irr}\left(G_{\vec{n}}\left(\mathbb{Q}_{p}\right)\right)$ be decomposed as

$$
\pi_{p}^{\vec{n}}=\bigotimes_{i=1}^{m} \pi_{\mathfrak{p}_{i}}^{\vec{n}} \otimes \pi_{0}^{\vec{n}}
$$

Assume that there is some $i$ such that $\pi_{\mathfrak{p}_{i}}^{\vec{n}}$ transfers to a generic principal series representation of $G L_{n}\left(F_{\mathfrak{p}_{i}}\right)$ and $J_{b_{i}}$ is a non-quasi-split inner form of $M_{b_{i}}$. Then

$$
\operatorname{Red}_{\vec{n}}^{b}\left(\pi_{p}\right)=0 .
$$

Proof. This follows from the explicit description of $\operatorname{Red}_{\vec{n}}^{b}$ above, which includes the Langlands-Jacquet map. If $\pi_{p}^{\vec{n}}$ satisfies the above condition, then its image $\rho$ in $\operatorname{Groth}\left(M_{b}\left(\mathbb{Q}_{p}\right)\right)$ will have as $M_{b_{i}}\left(F_{\mathfrak{p}_{i}}\right)$-components only generic principal series representations. Indeed, to see this, note that by the definition in Section 2 of Shi10, for a $G_{\vec{n}}$-endoscopic triple, the $L$-morphism ${ }^{L} M_{G_{\vec{n}}} \rightarrow{ }^{L} M_{b}$ is the restriction of the $L$-morphism $\tilde{\zeta}_{n_{1}, n_{2}}:{ }^{L} G_{n_{1}, n_{2}} \rightarrow{ }^{L} G_{n}$. The condition of being a generic principal series representation can be interpreted on the dual side, and is then easily deduced from this diagram. But if $\rho \in \operatorname{Groth}\left(M_{b}\left(\mathbb{Q}_{p}\right)\right)$ has only generic principal series representations as $M_{b_{i}}\left(F_{\mathfrak{p}_{i}}\right)$-components, then it lies in the kernel of the Langlands-Jacquet map whenever $J_{b_{i}}$ is a non-quasi-split inner form, by the construction of this map following Theorem 3.1 and Proposition 3.3 of [Bad07].

5.5. Generic principal series. Fix test functions $f^{S} \in \mathcal{H}^{\mathrm{ur}}\left(\mathbb{G}_{n}\left(\mathbb{A}^{S}\right)\right), f_{S_{\mathrm{fin}} \backslash\{p\}} \in$ $C_{c}^{\infty}\left(\mathbb{G}_{n}\left(\mathbb{A}_{S_{\text {fin }} \backslash\{p\}}\right)\right)$ and let $\phi^{S}, \phi_{S_{\text {fin }} \backslash\{p\}}$ be their base change transfers to $G_{n}\left(\mathbb{A}^{S}\right)$ and $G_{n}\left(\mathbb{A}_{S_{\text {fin } \backslash\{p\}}}\right)$ as defined in Section 5.3 .

Lemma 5.5.1. For any test function $f_{p} \in C_{c}^{\infty}\left(\mathbb{G}_{\mathbb{Q}}\left(\mathbb{Q}_{p}\right)\right)$, let $\phi_{p} \in C_{c}^{\infty}\left(G\left(\mathbb{Q}_{p}\right)\right)$ be its base change transfer. The trace

$$
\operatorname{tr}\left(\phi^{S} \phi_{S_{\mathrm{fin}} \backslash\{p\}} \phi_{p} \mid \iota_{\ell}\left(\left[H_{c}\left(\mathscr{I}_{\text {Mant }}^{b}, \overline{\mathbb{Q}}_{\ell}\right)\right]\right)\right)
$$

can be written as a linear combination of terms of the form

$$
\operatorname{tr}\left(\left(f^{\vec{n}}\right)^{S} \mid\left(\Pi^{\vec{n}}\right)^{S}\right) \operatorname{tr}\left(\left(f^{\vec{n}}\right)_{S_{\text {fin }} \backslash\{p\}} \mid\left(\Pi^{\vec{n}}\right)_{S_{\text {fin }} \backslash\{p\}}\right) \operatorname{tr}\left(\phi_{p} \mid \operatorname{Red}_{\vec{n}}^{b}\left(\pi_{p}^{\vec{n}}\right)\right)
$$

for $\pi_{p}^{\vec{n}} \in \operatorname{Irr}\left(G_{\vec{n}}\left(\mathbb{Q}_{p}\right)\right)$, $\Pi^{\vec{n}}$ satisfying the following condition. The representation $\pi_{p}^{\vec{n}}$ base changes to the component $\Pi_{p}^{\vec{n}} \in \operatorname{Irr}\left(\mathbb{G}_{\vec{n}}\left(\mathbb{Q}_{p}\right)\right)$ at $p$ of a $\theta$-stable isobaric automorphic representation $\Pi^{\vec{n}}$ of $\mathbb{G}_{\vec{n}}$ of the form

$$
\Pi^{\vec{n}}=\left(\mathrm{n}-\operatorname{Ind}_{Q}^{\mathbb{G}_{\vec{n}}} \Pi_{M}\right)_{\xi}
$$

where $\Pi_{M}$ occurs in the (relatively) discrete part of the automorphic spectrum of the Levi subgroup $M$ of a parabolic $Q \subset \mathbb{G}_{\vec{n}}$. Moreover, $\Pi_{\infty}^{\vec{n}}$ is cohomological (with respect to the trivial algebraic representation). 
Proof. We follow the proof of Proposition 6.1 of [Shi11, in a more general situation, but without keeping track of endoscopic signs and constants. First, assume that $f_{p}$ is chosen such that $\phi_{p}$ is acceptable. Let $\phi$ be the product of test functions $\phi^{S} \phi_{S_{\mathrm{fin} \backslash\{p\}}} \phi_{p}$. By combining Theorem 5.3.2 and Proposition 5.3.3, we can write $\operatorname{tr}\left(\phi \mid \iota_{\ell}\left[H_{c}\left(\mathscr{I}_{\text {Mant }}^{b}, \overline{\mathbb{Q}}_{\ell}\right)\right]\right)$ as a finite linear combination on terms of the form $\operatorname{tr}\left(\Pi^{\vec{n}}\left(f^{\vec{n}}\right) \circ A^{\prime}\right)$, where $\Pi^{\vec{n}}$ is a $\theta$-stable irreducible automorphic representation of $\mathbb{G}_{\vec{n}}$.

Recall that each $\Pi^{\vec{n}}$ is of the form $\left(\mathrm{n}-\operatorname{Ind}_{Q}^{\mathbb{G}_{\vec{n}}} \Pi_{M}\right)_{\xi}$, where $\Pi_{M}$ occurs in the (relatively) discrete part of the automorphic spectrum of the Levi subgroup $M$ of $\mathbb{G}_{\vec{n}}$. The fact that $\Pi^{\vec{n}}$ is $\theta$-stable follows from Remark 5.3.4 and the irreducibility of $\Pi^{\vec{n}}$ follows from the fact $\Pi_{M}$ is unitary and that, for general linear groups, any parabolic induction of a unitary representation is irreducible. Moreover, the representation $\Pi_{M}$ must be isobaric, since it contributes to the discrete spectrum of $M$. (This follows from the classification of automorphic representations occurring in the discrete spectrum of general linear groups due to Moeglin and Waldspurger, MW89. See, for example, Theorem 1.3.3 of [Art13] and the discussion below it.) Now the strong multiplicity one result due to Jacquet and Shalika (the main result of [JS81], see also Theorem 1.3.2 of [Art13]) implies that the string of Satake parameters outside the finite set $S$ determines $\Pi_{M}$. The parabolic induction $\Pi^{\vec{n}}$ is also isobaric, because it is irreducible, and therefore it is determined by $\left(\Pi^{\vec{n}}\right)^{S}$. To check that $\Pi_{\infty}^{\vec{n}}$ is cohomological (for the trivial representation), it is enough to determine the infinitesimal character of $\Pi_{\infty}^{\vec{n}}$, which can be done using the definition of the test functions at $\infty$.

Decompose the intertwiner $A^{\prime}$ as $\left(A^{\prime}\right)^{p} \cdot A_{p}^{\prime}$. Using the fact that $\Pi^{\vec{n}}$ is $\theta$-stable and that the base change map at $p$ is injective (since $p$ splits in the quadratic field $\mathcal{K})$, we can rewrite $\operatorname{tr}\left(\Pi_{p}^{\vec{n}}\left(f_{p}^{\vec{n}}\right) \circ A_{p}^{\prime}\right)$ as $\operatorname{tr} \pi_{p}^{\vec{n}}\left(\phi_{p}^{\vec{n}}\right)$, for some representation $\pi_{p}^{\vec{n}}$ in $\operatorname{Irr}\left(G_{\vec{n}}\left(\mathbb{Q}_{p}\right)\right)$ (at least up to a sign). Now, using Lemma 5.4.2, we can rewrite the latter as $\operatorname{tr} \operatorname{Red}_{\vec{n}}^{b}\left(\pi_{p}^{\vec{n}}\right)\left(\phi_{p}\right)$.

Keeping $\phi^{p}$ fixed, we have a formula for $\operatorname{tr}\left(\phi \mid \iota_{\ell}\left[H_{c}\left(\mathscr{I}_{\text {Mant }}^{b}, \overline{\mathbb{Q}}_{\ell}\right)\right]\right)$ as a finite linear combination of traces of $\phi_{p}$ against irreducible representations of $J_{b}\left(\mathbb{Q}_{p}\right)$. At this stage, we can take $\phi_{p}$ to be any smooth, compactly-supported function on $J_{b}\left(\mathbb{Q}_{p}\right)$, not necessarily an acceptable one. Indeed, recall that the twist of any such $\phi^{p}$ by any sufficiently high power of Frobenius is acceptable, so the equality above holds for $\phi_{p}^{(N)}$ for sufficiently large $N$. The argument in the proof of Lemma 6.4 of [Shi09] now proves that the desired equality holds for every integer $N$ and, in particular, for $N=0$.

Remark 5.5.2. As a consequence, we see that the $\mathbb{G}_{n}\left(\mathbb{A}_{f}^{p}\right)$-representation

$$
B C^{p}\left(\left[H_{c}\left(\mathscr{I}_{\text {Mant }}^{b}, \overline{\mathbb{Q}}_{\ell}\right)\right]^{S \text { ur }}\right)
$$

can be written in terms of the transfer to $\mathbb{G}_{n}\left(\mathbb{A}_{f}^{p}\right)$ of representations $\left(\Pi^{\vec{n}}\right)_{f}^{p}$, where the $\Pi^{\vec{n}}$ are $\theta$-stable automorphic representations of $\mathbb{G}_{\vec{n}}$ as in the statement of the lemma, which are unramified outside $S$.

Moreover, recall the existence of Galois representations in the conjugate self-dual case. 
Theorem 5.5.3. Let $\Pi^{\vec{n}}$ be an isobaric automorphic representation of $\mathbb{G}_{\vec{n}}$, unramified outside $S$, of the form

$$
\Pi^{\vec{n}}=\left(\mathrm{n}-\operatorname{Ind}_{Q}^{\mathbb{G}_{\vec{n}}} \Pi_{M}\right)_{\xi},
$$

where $\Pi_{M}$ occurs in the (relatively) discrete part of the automorphic spectrum of the Levi subgroup $M$ of a parabolic $Q \subset \mathbb{G}_{\vec{n}}$ and is $\Phi_{\vec{n}}^{-1} \theta$-stable, with $\Pi_{\infty}^{\vec{n}}$ cohomological. Then there exists a conjugate self-dual (up to twist) continuous semisimple Galois representation

$$
r_{\Pi^{\vec{n}}, \ell}: \operatorname{Gal}(\bar{F} / F) \rightarrow G L_{\vec{n}}\left(\overline{\mathbb{Q}}_{\ell}\right)
$$

which is unramified outside $S \cup\{\ell\}$, and such that for all primes $q \neq \ell$ of $\mathbb{Q}$, in the decomposition

$$
\Pi_{q}^{\vec{n}}=\left(\bigotimes_{\mathfrak{q} \mid q} \Pi_{\mathfrak{q}}^{\vec{n}}\right) \otimes \chi_{q}
$$

corresponding to

$$
\mathbb{G}_{\vec{n}}\left(\mathbb{Q}_{p}\right)=\left(\prod_{\mathfrak{q} \mid q} G L_{\vec{n}}\left(F_{\mathfrak{q}}\right)\right) \times \mathcal{K}_{q}^{\times},
$$

the representations $\Pi_{\mathfrak{q}}^{\vec{n}}$ and $\left.r_{\Pi^{\vec{n}}, \ell}\right|_{\mathrm{Gal}\left(\bar{F}_{\mathfrak{q}} / F_{\mathfrak{q}}\right)}$ correspond under the local Langlands correspondence (up to Frobenius semisimplification) 28

Proof. Using the classification of the discrete spectrum of general linear groups due to Moeglin and Waldspurger, MW89, this follows from the main theorems of [Shi11, CH13] and Car12. We remark that the conjugate self-dual, regular algebraic case suffices here because $\Pi_{M}$ is $\Phi_{\vec{n}}^{-1} \theta$-stable and has regular infinitesimal character (since $\Pi^{\vec{n}}$ has regular infinitesimal character).

In the following, we fix a Galois representation

$$
r: \operatorname{Gal}(\bar{F} / F) \rightarrow G L_{n}\left(\overline{\mathbb{Q}}_{\ell}\right)
$$

which is unramified outside $S \cup\{\ell\}$, and restrict attention to the summand

$$
B C^{p}\left(\left[H_{c}\left(\mathscr{I}_{\text {Mant }}^{b}, \overline{\mathbb{Q}}_{\ell}\right)\right]^{S \text { ur }}\right)_{r}^{S \text { ur }} \text { of } B C^{S}\left(\left[H_{c}\left(\mathscr{I}_{\text {Mant }}^{b}, \overline{\mathbb{Q}}_{\ell}\right)\right]^{S \text { ur }}\right)
$$

coming from representations $\Pi^{\vec{n}}$ as above, with $r_{\Pi^{\vec{n}}, \ell} \cong r$ (under the embedding $\left.G L_{\vec{n}}\left(\overline{\mathbb{Q}}_{\ell}\right) \hookrightarrow G L_{n}\left(\overline{\mathbb{Q}}_{\ell}\right)\right)$.

The following theorem is the key result of this section. Recall that we have fixed a prime $\mathfrak{p} \mid p$ of the reflex field $E$, so that we have embeddings $E \hookrightarrow \mathbb{C}, E \hookrightarrow E_{\mathfrak{p}} \hookrightarrow$ $\overline{\mathbb{Q}}_{p}$. For convenience, let us fix an isomorphism $\iota_{p}: \overline{\mathbb{Q}}_{p} \cong \mathbb{C}$ compatible with the embedding of $E$.

Theorem 5.5.4. For each prime $\mathfrak{p}_{i}$ of $F$, let

$$
S_{i}=\left\{\tau: F \hookrightarrow \mathbb{C} \mid \iota_{p} \circ \tau \text { induces } \mathfrak{p}_{\mathfrak{i}}\right\} .
$$

Assume that for each $i, S_{i}$ contains at most one $\tau$ for which $p_{\tau} q_{\tau}$ is nonzero, where $G$ has signature $\left(p_{\tau}, q_{\tau}\right)$ at $\tau: F \hookrightarrow \mathbb{C}$. Moreover, for each $i$ for which $S_{i}$ contains some $\tau$ for which $p_{\tau} q_{\tau}$ is nonzero, assume that

$$
r_{\mathrm{Gal}\left(\bar{F}_{\mathfrak{p}_{i}} / F_{\mathfrak{p}_{i}}\right)}=\chi_{i, 1} \oplus \ldots \oplus \chi_{i, n}
$$

\footnotetext{
${ }^{28}$ For our purposes, it is enough to know the compatibility up to semisimplification, i.e. without identification of the monodromy operator, which is the most subtle part of the local-globalcompatibility.
} 
decomposes as a direct sum of characters, such that for all $a \neq b, \chi_{i, a} \chi_{i, b}^{-1}$ is not the cyclotomic character.

Then, if $b \in B\left(G, \mu^{-1}\right)$ is not $\mu$-ordinary 29

$$
B C^{p}\left(\left[H_{c}\left(\mathscr{I}_{\text {Mant }}^{b}, \overline{\mathbb{Q}}_{\ell}\right)\right]^{\text {Sur }}\right)_{r}=0 .
$$

Proof. Assume the contrary. Then there is some $\theta$-stable isobaric automorphic representation $\Pi^{\vec{n}}$ of $\mathbb{G}_{\vec{n}}$ as above, with $r_{\Pi_{\vec{n}}, \ell} \cong r$, which contributes to $B C^{S}\left(\left[H_{c}\left(\mathscr{I}_{\text {Mant }}^{b}, \overline{\mathbb{Q}}_{\ell}\right)\right]^{S \text { ur }}\right)$. The component $\Pi_{p}^{\vec{n}}$ of $\Pi^{\vec{n}}$ at $p$ comes from a unique representation $\pi_{p}^{\vec{n}} \in \operatorname{Irr}\left(G_{\vec{n}}\left(\mathbb{Q}_{p}\right)\right)$ via base change. We may decompose

$$
\pi_{p}^{\vec{n}}=\bigotimes_{i=1}^{m} \pi_{\mathfrak{p}_{i}}^{\vec{n}} \otimes \pi_{0}^{\vec{n}}
$$

according to

$$
G_{\vec{n}}\left(\mathbb{Q}_{p}\right)=\prod_{i=1}^{m} G L_{\vec{n}}\left(F_{\mathfrak{p}_{i}}\right) \times \mathbb{Q}_{p}^{\times} .
$$

By the assumption on $r$ and local-global compatibility, we know that $\pi_{\mathfrak{p}_{i}}^{\vec{n}}$ transfers to a generic principal series representation of $G L_{n}\left(F_{\mathfrak{p}_{i}}\right)$ for all $i$ for which $S_{i}$ contains some $\tau$ with $p_{\tau} q_{\tau} \neq 0$. By Lemma 5.4.3. $\operatorname{Red}_{\vec{n}}^{b}\left(\pi_{p}^{\vec{n}}\right)=0$ as soon as $J_{b_{i}}$ is not quasisplit for some such $i$, so that in this case there is no contribution by Lemma 5.5.1]

It remains to see that if $b \in B\left(G, \mu^{-1}\right)$ is not $\mu$-ordinary, then there is some $i$ for which $S_{i}$ contains some $\tau$ with $p_{\tau} q_{\tau} \neq 0$, such that $J_{b_{i}}$ is not quasisplit.

We can decompose

$$
\mu=\left(\left(\mu_{i}\right)_{i=1, \ldots, m}, \mu_{0}\right): \mathbb{G}_{m} \rightarrow G_{\overline{\mathbb{Q}}_{p}}=\prod_{i=1}^{m}\left(\prod_{F_{\mathfrak{p}_{i}} \hookrightarrow \overline{\mathbb{Q}}_{p}} G L_{n, \overline{\mathbb{Q}}_{p}}\right) \times \mathbb{G}_{m, \overline{\mathbb{Q}}_{p}} ;
$$

let $G_{i}=\operatorname{Res}_{F_{\mathbf{p}_{i}} / \mathbb{Q}_{p}} G L_{n}$. Then $\mu_{i}$ is a conjugacy class of minuscule cocharacters of $G_{i}$, and we have a decomposition

$$
B\left(G, \mu^{-1}\right)=\prod_{i=1}^{m} B\left(G_{i}, \mu_{i}^{-1}\right),
$$

as the $\mathbb{G}_{m}$ factor plays no role here. In each factor $G L_{n, \overline{\mathbb{Q}}_{p}}, \mu$ has the form

$$
t \mapsto \operatorname{diag}(t, \ldots, t, 1, \ldots, 1)
$$

with $t$ occuring $p_{\tau}$ times, and 1 occuring $q_{\tau}$ times, where $\tau: F \rightarrow \overline{\mathbb{Q}}_{p} \cong \mathbb{C}$ is the corresponding complex place. In particular, for each $i$ for which $S_{i}$ does not contain any $\tau$ with $p_{\tau} q_{\tau} \neq 0, \mu_{i}$ is central, which implies that $B\left(G_{i}, \mu_{i}^{-1}\right)$ has precisely one element. If there is exactly one such $\tau$, then denoting by $\mu_{i, \tau}$ the corresponding component of $\mu_{i}$, one sees that

$$
B\left(G_{i}, \mu_{i}^{-1}\right)=B\left(G L_{n} / F_{\mathfrak{p}_{i}}, \mu_{i, \tau}^{-1}\right),
$$

using the relative $B(H / L)=B(L, H)$ for a reductive group $H$ over a $p$-adic field $L 30$ Now the result follows from the next lemma.

\footnotetext{
${ }^{29}$ It might be more accurate to write $\mu^{-1}$-ordinary.

${ }^{30}$ So far, we were only using the case $L=\mathbb{Q}_{p}$, and did not include this in the notation.
} 
Lemma 5.5.5. Let $L$ be any $p$-adic field, and let

$$
\mu: \mathbb{G}_{m} \rightarrow G L_{n}: t \mapsto \operatorname{diag}(t, \ldots, t, 1, \ldots, 1)
$$

be a minuscule cocharacter with $n-q$ occurences of $t$ and $q$ occurences of 1 . Then there is exactly one element $b \in B\left(G L_{n} / L, \mu^{-1}\right)$ for which $J_{b}$ is quasisplit, namely the $\mu$-ordinary element represented by $\operatorname{diag}\left(\varpi^{-1}, \ldots, \varpi^{-1}, 1, \ldots, 1\right)$, with $n-q$ occurences of the uniformizer $\varpi$ of $L$, and $q$ occurences of 1 .

Proof. By the choice of $\mu$, we know that for any $b \in B\left(G L_{n} / L, \mu^{-1}\right)$, the slopes $\lambda_{i}$ satisfy $-1 \leq \lambda_{i} \leq 0$. If some slope $\lambda$ is nonintegral, then $J_{b}$ is not quasisplit, as it contains a factor which is a general linear group over the division algebra of invariant $\lambda(\bmod 1)$ over $L$. Thus, if $J_{b}$ is quasisplit, then all slopes are equal to 0 or -1 ; from the equality $\kappa(b)=-\mu$ one deduces that slope -1 occurs with multiplicity $n-q$, and slope 0 with multiplicity $q$, which corresponds to the $\mu$-ordinary element $b=\operatorname{diag}\left(\varpi^{-1}, \ldots, \varpi^{-1}, 1, \ldots, 1\right)$. For this $b, J_{b} \cong G L_{n-q} \times G L_{q}$ is quasisplit.

5.6. Simple Shimura varieties. In this section, we sketch how to adapt the arguments above for Kottwitz' simple Shimura varieties as in Kot92a. This includes the case of Shimura varieties which admit $q$-adic uniformization, for some rational prime $q$ distinct from $p$ and $\ell$. In that case, our main result is related to level-raising results, as shown in Tho14.

Recall that $F=F^{+} \cdot \mathcal{K}$. Assume that we have a PEL datum of the form $(B, *, V,\langle\rangle, h$,$) , where B$ is a division algebra with center $F, V$ is a simple $B$ module, and $*$ is an involution of the second kind. Then the corresponding Shimura varieties $S_{K}$ are proper and the group $G$ has no endoscopy. Assume that $B$ is split at all places over $p$, in which case the constructions and results of Section 5.4 carry over. However, Theorem 5.2 .3 simplifies considerably. We follow Section 6 of [Shi12, where it is assumed that $p$ is inert in $F^{+}$; this assumption is not necessary for our purposes. As above, let $G_{n}$ be a quasi-split inner form of $G$ over $\mathbb{Q}$ and fix an isomorphism $G_{n} \simeq G$ over $\mathbb{Q}_{p}$.

Proposition 5.6.1. Let $\phi=\phi^{p} \phi_{p} \in \mathcal{C}_{c}^{\infty}\left(G\left(\mathbb{A}_{f}^{p}\right) \times J_{b}\left(\mathbb{Q}_{p}\right)\right)$, with $\phi_{p}$ an acceptable function. Then

$$
\operatorname{tr}\left(\phi \mid \iota_{\ell} H_{c}\left(\mathscr{I}_{\text {Mant }}^{b}, \overline{\mathbb{Q}} \ell_{\ell}\right)\right)=\left|\operatorname{ker}^{1}(\mathbb{Q}, G)\right| \iota\left(G, G_{n}\right) S T_{e}^{G_{n}}\left(\phi^{G_{n}}\right) .
$$

Proof. The other terms in the stable trace formula vanish by Lemma 7.1 of [Shi10].

We can now combine this with the stable trace formula for the $S_{K}$, which is Theorem 6.1 of [Art89] and which is simplified in our situation as in Proposition 6.3 of [Shi12, also making use of Lemma 5.4 .2 for $G_{n}\left(\mathbb{Q}_{p}\right) \simeq G\left(\mathbb{Q}_{p}\right)$. We get

$$
\operatorname{Red}_{n}^{b}\left(\left[H\left(S_{K}, \overline{\mathbb{Q}}_{\ell}\right)\right]\right)=\epsilon_{G} \cdot d\left(G_{\mathbb{R}}\right) \cdot\left[H_{c}\left(\mathscr{I}_{\text {Mant }}^{b}, \overline{\mathbb{Q}}_{\ell}\right)\right],
$$

where $\epsilon_{G}, d\left(G_{\mathbb{R}}\right)$ are certain non-zero constants. Again, we appeal to Lemma 6.4 of [Shi11] to extend a trace identity from acceptable $\phi_{p}$ to all $\phi_{p} \in \mathcal{C}_{c}^{\infty}\left(J_{b}\left(\mathbb{Q}_{p}\right)\right)$. We combine this with Matsushima's formula, which gives a description of $\left[\iota_{\ell} H\left(S_{K}, \overline{\mathbb{Q}}_{\ell}\right)\right]$ in terms of automorphic representations of $G$. We get an analogue of Corollary 6.12 of Shi12.

Corollary 5.6.2. We have the following equality in $\operatorname{Groth}\left(G\left(\mathbb{A}_{f}^{p}\right) \times J_{b}\left(\mathbb{Q}_{p}\right)\right)$ :

$$
\left[\iota_{\ell} H_{c}\left(\mathscr{I}_{\text {Mant }}^{b}, \overline{\mathbb{Q}}_{\ell}\right)\right]=(-1)^{q(G)} \sum_{\pi_{f}} c\left(\pi_{f}\right)\left[\pi_{f}^{p}\right]\left[\operatorname{Red}_{n}^{b}\left(\pi_{p}\right)\right] .
$$


The sum runs over admissible representations $\pi_{f}$ of $G\left(\mathbb{A}_{f}\right)$ such that $\pi_{f} \pi_{\infty}$ is an automorphic representation of $G$, for some representation $\pi_{\infty}$ of $G(\mathbb{R})$ which is cohomological for the trivial algebraic representation. The coefficients $c\left(\pi_{f}\right)$ are related to the automorphic multiplicity of $\pi_{f} \pi_{\infty}$.

In this case, the existence of Galois representations is also known, as the stable base change of such $\pi$ to $G L_{n}$ has been established by Shin in the appendix to Gol14. As before, for a Galois representation

$$
r: \operatorname{Gal}(\bar{F} / F) \rightarrow G L_{n}\left(\overline{\mathbb{Q}}_{\ell}\right),
$$

we restrict attention to the summand $\left[H_{c}\left(\mathscr{I}_{\text {Mant }}^{b}, \overline{\mathbb{Q}}_{\ell}\right)\right]_{r}$ of

$$
\left[H_{c}\left(\mathscr{I}_{\text {Mant }}^{b}, \overline{\mathbb{Q}}_{\ell}\right)\right]
$$

coming from representations $\pi$ as above, with $r_{\pi, \ell} \cong r$.

We get the following analogue of Theorem 5.5.4, which is proved in the same way.

Corollary 5.6.3. For each prime $\mathfrak{p}_{i}$ of $F$ above $p$, let

$$
S_{i}=\left\{\tau: F \hookrightarrow \mathbb{C} \mid \iota_{p} \circ \tau \text { induces } \mathfrak{p}_{\mathfrak{i}}\right\} .
$$

Assume that for each $i, S_{i}$ contains at most one $\tau$ for which $p_{\tau} q_{\tau}$ is nonzero, where $G$ has signature $\left(p_{\tau}, q_{\tau}\right)$ at $\tau: F \hookrightarrow \mathbb{C}$. Moreover, for each $i$ for which $S_{i}$ contains some $\tau$ for which $p_{\tau} q_{\tau}$ is nonzero, assume that

$$
r_{\operatorname{Gal}\left(\bar{F}_{\mathfrak{p}_{i}} / F_{\mathfrak{p}_{i}}\right)}=\chi_{i, 1} \oplus \ldots \oplus \chi_{i, n}
$$

decomposes as a direct sum of characters, such that for all $a \neq b, \chi_{i, a} \chi_{i, b}^{-1}$ is not the cyclotomic character.

Then, if $b \in B\left(G, \mu^{-1}\right)$ is not $\mu$-ordinary,

$$
\left[H_{c}\left(\mathscr{I}_{\text {Mant }}^{b}, \overline{\mathbb{Q}}_{\ell}\right)\right]_{r}=0 .
$$




\section{TORsion In the COHOMOlOgY OF Unitary Shimura VARIETIES}

In this final section, we give a precise formulation and proof of our main result. We start by formulating and proving the critical perversity result.

6.1. Perverse sheaves on the flag variety. Consider the Hodge-Tate period map

$$
\pi_{H T}: \mathcal{S}_{K^{p}} \rightarrow \mathscr{F} \ell_{G, \mu}
$$

for a compact Hodge type Shimura variety. In this section, we would like to make precise in which sense $R \pi_{H T *} \mathbb{F}_{\ell}$ is perverse 31

Recall the following result on preservation of perversity under nearby cycles.

Theorem 6.1.1 (Il194, Corollaire 4.5]). Let $K$ be a complete nonarchimedean field with ring of integers $\mathcal{O}_{K}$ and completed algebraic closure $C$ with $\mathcal{O}_{C} \subset C$, and let $\ell$ be a prime which is invertible in $\mathcal{O}_{K}$. Let $X$ be a scheme of finite type over $\mathcal{O}_{K}$. Let $X_{\mathcal{O}_{C}}$ be the base-change to $\mathcal{O}_{C}$, with geometric generic fibre $j: X_{\bar{\eta}}=$ $X_{\mathcal{O}_{K}} \otimes_{\mathcal{O}_{K}} C \hookrightarrow X_{\mathcal{O}_{C}}$ and geometric special fibre $i: X_{\bar{s}} \hookrightarrow X_{\mathcal{O}_{C}}$. Let $\mathscr{F}$ be a

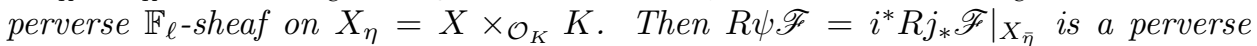
sheaf on $X_{\bar{s}}$.

Moreover, nearby cycles in the scheme setting agree with nearby cycles in the formal/rigid setting. More precisely, we have the following result.

Theorem 6.1.2 ([Hub96, Theorem 3.5.13]). Let the situation be as in Theorem 6.1.1. Let $\mathcal{X}_{\eta}$ be the associated rigid-analytic variety over $K$, considered as an adic space, with base change $\mathcal{X}_{\bar{\eta}}$ to $C$. There is a natural morphism of sites $\lambda: \mathcal{X}_{\bar{\eta} \text {,ét }} \rightarrow\left(X_{\bar{s}}\right)_{\text {ét }}$, given by lifting an étale map $Y \rightarrow X_{\bar{s}}$ to an étale map of formal schemes over $\mathcal{O}_{C}$, and then taking the generic fibre.

Let $\mathscr{F}^{\text {ad }}$ be the pullback of $\mathscr{F}$ under $\mathcal{X}_{\text {ét }} \rightarrow X_{\text {ét }}$. Then

$$
R \lambda_{*}\left(\mathscr{F}^{\mathrm{ad}}{\mid \mathcal{X}_{\bar{\eta}}} \cong R \psi \mathscr{F} .\right.
$$

In our situation, it is hard to give a direct definition of perversity of $R \pi_{H T *} \mathbb{F}_{\ell}$. However, the above properties suggest that at least, for every formal model $X$ of the flag variety $\mathscr{F} \ell_{G, \mu}$, the nearby cycles $R \psi_{X} R \pi_{H T *} \mathbb{F}_{\ell}$ should be a perverse sheaf on the special fibre $X_{\bar{s}}$ of $X$. This is still not true, as $G\left(\mathbb{Q}_{p}\right)$ acts on $R \pi_{H T *} \mathbb{F}_{\ell}$; one can only hope for the $K_{p}$-invariants to be perverse, for any sufficiently small $K_{p} \subset G\left(\mathbb{Q}_{p}\right)$. Thus, we work with the equivariant sites introduced in Sch15a, §2].

First, note that $R \pi_{H T *} \mathbb{F}_{\ell}$ is a canonically a complex of sheaves on the equivariant site $\left(\mathscr{F} \ell_{G, \mu} / G\left(\mathbb{Q}_{p}\right)\right)_{\text {ét }}$. More precisely, one has the map of equivariant sites

$$
\pi_{H T} / G\left(\mathbb{Q}_{p}\right):\left(\mathcal{S}_{K^{p}} / G\left(\mathbb{Q}_{p}\right)\right)_{\text {ét }} \rightarrow\left(\mathscr{F} \ell_{G, \mu} / G\left(\mathbb{Q}_{p}\right)\right)_{\text {ét }},
$$

and one can look at $R\left(\pi_{H T} / G\left(\mathbb{Q}_{p}\right)\right)_{\text {ét } *} \mathbb{F}_{\ell}$, and this pulls back to $R \pi_{H T *} \mathbb{F}_{\ell}$ under the projection $\left(\mathscr{F} \ell_{G, \mu}\right)_{\text {ét }} \rightarrow\left(\mathscr{F} \ell_{G, \mu} / G\left(\mathbb{Q}_{p}\right)\right)_{\text {ét }}$. To check the latter statement, note first that by passing to slice categories, using [Sch15a, Proposition 2.9], one may replace $G\left(\mathbb{Q}_{p}\right)$ by any compact open subgroup $K_{p} \subset G\left(\mathbb{Q}_{p}\right)$, and then one can pass to the limit using Sch15a, Proposition 2.8].

Now take any étale $U=\operatorname{Spa}\left(A, A^{\circ}\right) \rightarrow \mathscr{F} \ell_{G, \mu}$. By [Sch15a, Corollary 2.5], the action of $K_{p}$ extends to a continuous action on $U$ if $K_{p}$ is sufficiently small. Let

\footnotetext{
${ }^{31}$ As we are far from a finite type situation, we avoid talking about $\mathbb{Q}_{\ell}$-sheaves. We could talk about $\mathbb{Z} / \ell^{n} \mathbb{Z}$-sheaves, but in that case the notion of perversity is slightly subtle as $\mathbb{Z} / \ell^{n} \mathbb{Z}$ is not a field. For our applications, the $\mathbb{F}_{\ell^{-}}$case is enough.
} 
$\mathfrak{U}=\operatorname{Spf}\left(A^{\circ}\right)$ with special fibre $\mathfrak{U}_{s}=\operatorname{Spec}\left(A^{\circ} / p\right)$. Then $K_{p}$ acts trivially on $\mathfrak{U}_{s}$ if $K_{p}$ is sufficiently small, by continuity of the $K_{p}$-action and finite generation of $A^{\circ} / p$. It follows that any étale map to $\mathfrak{U}_{\bar{s}}$ lifts to a $K_{p}$-equivariant étale map to $\mathfrak{U}_{\mathcal{O}_{C}}$ (where $C=\mathbb{C}_{p}$ ), giving a natural morphism of sites

$$
\lambda_{U / K_{p}}:\left(U_{\bar{\eta}} / K_{p}\right)_{\text {ét }} \rightarrow \mathfrak{U}_{\bar{s}, \text { ét }} .
$$

Proposition 6.1.3. Let

$$
\pi_{H T}: \mathcal{S}_{K^{p}} \rightarrow \mathscr{F} \ell_{G, \mu}
$$

be the Hodge-Tate period map for a compact Shimura variety of Hodge type and any sufficiently small compact open subgroup $K^{p} \subset G\left(\mathbb{A}_{f}^{p}\right)$. Let $\bar{x} \in \mathscr{F} \ell_{G, \mu}$ be a geometric point. Then there exists a neighborhood basis of affinoid étale neighborhoods $U=\operatorname{Spa}\left(A, A^{\circ}\right)$ of $x$ in $\mathscr{F} \ell_{G, \mu}$ such that, denoting $\mathfrak{U}=\operatorname{Spf}\left(A^{\circ}\right)$,

$$
\left.R \lambda_{U / K_{p} *}\left(R\left(\pi_{H T} / G\left(\mathbb{Q}_{p}\right)\right)_{*} \mathbb{F}_{\ell}\right)\right|_{U_{\bar{\eta}} / K_{p}}[\langle 2 \rho, \mu\rangle]
$$

is a perverse sheaf on $\mathfrak{U}_{\bar{s}}$ for any sufficiently small pro-p compact open subgroup $K_{p} \subset G\left(\mathbb{Q}_{p}\right)$.

Proof. By [Sch15b, Theorem IV.1.1 (i)], one can find some affinoid étale (in fact, open) neighborhood $U$ of $x$ such that $\mathcal{S}_{K^{p}, U}=\mathcal{S}_{K^{p}} \times \mathscr{F}_{G, \mu} U$ is affinoid perfectoid, and equal to the preimage of an affinoid étale $\mathcal{S}_{K_{p} K^{p}, U} \rightarrow \mathcal{S}_{K_{p} K^{p}}$ for any sufficiently small $K_{p}$. These properties will then also be true for any étale $V \rightarrow U$ that factors as a composite of finite étale maps and rational embeddings, and such $V$ are cofinal. Thus, fix any $U$ with the stated properties.

Let

$$
\pi_{H T, U}: \mathcal{S}_{K^{p}, U} \rightarrow U=\operatorname{Spa}\left(A, A^{\circ}\right)
$$

be the restriction of $\pi_{H T}$. As $\pi_{H T}$ is partially proper, so is $\pi_{H T, U}$. If $K_{p}$ is sufficiently small, $\pi_{H T, U}$ is $K_{p}$-equivariant, and induces a map

$$
\pi_{H T, U / K_{p}}: \mathcal{S}_{K^{p}, U} / K_{p} \rightarrow U / K_{p} .
$$

Also

$$
\left.\left(R\left(\pi_{H T} / G\left(\mathbb{Q}_{p}\right)\right)_{*} \mathbb{F}_{\ell}\right)\right|_{U / K_{p}}=R \pi_{H T, U / K_{p} *} \mathbb{F}_{\ell},
$$

and by [Sch15a, Proposition 2.12], there is an equivalence of sites $\left(\mathcal{S}_{K^{p}, U} / K_{p}\right)_{\text {ét }} \cong$ $\mathcal{S}_{K_{p} K^{p}, U \text {,ét }}$.

Now any $\mathcal{S}_{K_{p} K^{p}, U}=\operatorname{Spa}\left(R_{K_{p} K^{p}, U}, R_{K_{p} K^{p}, U}^{\circ}\right)$ has its natural integral model $\mathfrak{S}_{K_{p} K^{p}, U}=\operatorname{Spf}\left(R_{K_{p} K^{p}, U}^{\circ}\right)$, with inverse limit $\mathfrak{S}_{K^{p}, U}=\operatorname{Spf}\left(R_{K^{p}, U}^{\circ}\right)$, where $\mathcal{S}_{K^{p}, U}=$ $\operatorname{Spa}\left(R_{K^{p}, U}, R_{K^{p}, U}^{\circ}\right)$. We get a map of formal schemes

$$
\pi_{H T, \mathfrak{U}}: \mathfrak{S}_{K^{p}, U} \rightarrow \mathfrak{U} .
$$

Modulo $p$, we get a map of schemes

$$
\pi_{H T, \mathfrak{U}_{s}}: \mathfrak{S}_{K^{p}, U, s} \rightarrow \mathfrak{U}_{s}
$$

with $\mathfrak{S}_{K^{p}, U, s}=\operatorname{Spec}\left(R_{K^{p}, U}^{\circ} / p\right)$, and $\mathfrak{U}_{s}=\operatorname{Spec}\left(A^{\circ} / p\right)$. But $\mathfrak{U}_{s}$ is of finite type over $\mathbb{F}_{p}$, and $\mathfrak{S}_{K^{p}, U, s}=\lim _{K_{p}} \mathfrak{S}_{K_{p} K^{p}, U, s}$ in the category of (affine) schemes. It follows that $\pi_{H T, \mathfrak{U}_{s}}$ factors over a map

$$
\pi_{H T, K_{p}, \mathfrak{U}_{s}}: \mathfrak{S}_{K_{p} K^{p}, U, s} \rightarrow \mathfrak{U}_{s}
$$

(of affine schemes of finite type over $\mathbb{F}_{p}$ ) for any sufficiently small $K_{p}$. We claim that $\pi_{H T, K_{p}, \mathfrak{U}_{s}}$ satisfies the valuative criterion of properness. If $K$ is an algebraically closed field with a rank-1-valuation ring $V \subset K$, and we are given a $V$-point of $\mathfrak{U}_{s}$ together with a lift of the corresponding $K$-valued point to a $K$-valued point of 
$\mathfrak{S}_{K_{p} K^{p}, U, s}$, we need to show that this $K$-valued point is in fact $V$-valued. We may lift the $K$-valued point of $\mathfrak{S}_{K_{p} K^{p}, U, s}$ to $\mathfrak{S}_{K^{p}, U, s}$ (as all transition maps are finite and surjective). We may then find a complete algebraically closed extension $C / \mathbb{Q}_{p}$ with residue field $K$ and a $\left(C, \mathcal{O}_{C}\right)$-valued point of $\mathcal{S}_{K^{p}, U}$ specializing to this $K$ valued point of $\mathfrak{S}_{K^{p}, U, s}$. Let $C^{+} \subset \mathcal{O}_{C}$ be the preimage of $V \subset K$. Then the image of the $\left(C, \mathcal{O}_{C}\right)$-valued point of $\mathcal{S}_{K^{p}, U}$ under $\pi_{H T, U}$ is a $\left(C, \mathcal{O}_{C}\right)$-valued point of $U$ which extends to a $\left(C, C^{+}\right)$-valued point. As $\pi_{H T, U}$ is partially proper, it follows that we get a $\left(C, C^{+}\right)$-valued point of $\mathcal{S}_{K^{p}, U}$, which specializes to a $V$-valued point of $\mathfrak{S}_{K^{p}, U, s}$ and thus of $\mathfrak{S}_{K_{p} K^{p}, U, s}$, as desired.

Thus, $\pi_{H T, K_{p}, \mathfrak{U}_{s}}$ is a map of affine schemes of finite type over $\mathbb{F}_{p}$ which satisfies the valuative criterion of properness, i.e., it is finite 32 Now consider the following diagram, where we have base-changed some spaces and maps to algebraically closed fields.

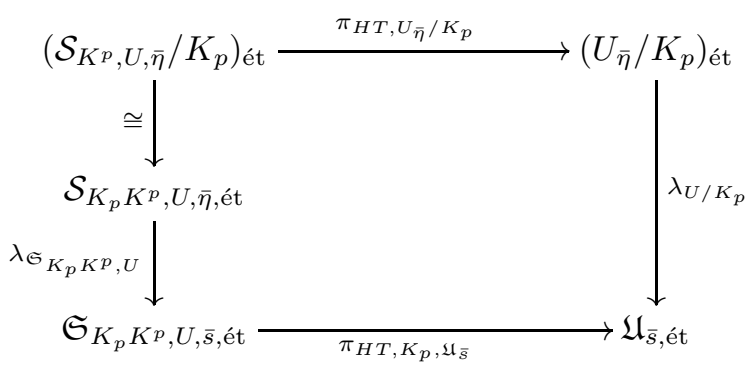

We are interested in the pushforward of $\mathbb{F}_{\ell}$ from the upper left to the lower right corner, computed via the upper right corner. We may equivalently compute it via the lower left corner. In that case, the first pushforward is perverse by Theorem 6.1.1 and Theorem 6.1.2 up to the shift $\langle 2 \rho, \mu\rangle=\operatorname{dim} \mathcal{S}_{K_{p} K^{p}, U}$. But $\pi_{H T, K_{p}, \mathfrak{U}_{\bar{s}}}$ is finite, so it also preserves perversity under pushforward.

We will need the following consequence, which is a statement purely about the cohomology of Igusa varieties. For the statement, let $S$ be a finite set of primes such that $K^{p}=K_{S}^{p} K^{S}$, where $K^{S} \subset G\left(\mathbb{A}_{f}^{S}\right)$ is a product of hyperspecial maximal compact open subgroups, and $K_{S}^{p} \subset G\left(\mathbb{A}_{S}^{p}\right)$. Let

$$
\mathbb{T}^{S}=Z\left[G\left(\mathbb{A}_{f}^{S}\right) / / K^{S}\right]
$$

be the abstract (commutative) Hecke algebra of $K^{S}$-biinvariant compactly supported functions on $G\left(\mathbb{A}_{f}^{S}\right)$.

Corollary 6.1.4. Fix a maximal ideal $\mathfrak{m} \subset \mathbb{T}^{S}$, and among all $b \in B\left(G, \mu^{-1}\right)$ with the property that the $\mathfrak{m}$-torsion

$$
H^{i}\left(\operatorname{Ig}^{b}, \mathbb{F}_{\ell}\right)[\mathfrak{m}] \neq 0
$$

for some $i \in \mathbb{Z}$, take some $b$ with $d=\left\langle 2 \rho, \nu_{b}\right\rangle$ minimal. Then $H^{i}\left(\operatorname{Ig}^{b}, \mathbb{F}_{\ell}\right)[\mathfrak{m}]$ is nonzero only for $i=d$.

The idea is that the sheaf $\left(R \pi_{H T *} \mathbb{F}_{\ell}\right)_{\mathfrak{m}}$ is concentrated on a subset of dimension $\langle 2 \rho, \mu\rangle-d$ by assumption. Thus, $\mathscr{F} \ell_{G, \mu}^{b}$ is one of the largest strata where $\left(R \pi_{H T *} \mathbb{F}_{\ell}\right)_{\mathfrak{m}}$ is nonzero. But as this sheaf is (up to shift) perverse, one concludes by observing that on the largest stratum where a perverse sheaf is nonzero, it is

\footnotetext{
${ }^{32}$ Thus, we are in the somewhat curious situation that $\pi_{H T, \mathfrak{U}_{s}}$ is ind-finite, but $\pi_{H T, U}$ has fibres of positive dimension.
} 
concentrated in one degree. However, as the notion of perversity is defined via nearby cycles, we need to rewrite this argument slightly.

Proof. As

$$
H^{i}\left(\operatorname{Ig}^{b}, \mathbb{F}_{\ell}\right)=\underset{m}{\lim } H^{i}\left(\mathscr{I}_{\text {Mant }, m}^{b}, \mathbb{F}_{\ell}\right),
$$

where the transition maps are split injective (namely, projections are given by averaging operators over compact open subgroups of $J_{b}\left(\mathbb{Q}_{p}\right)$ ), and the terms on the right are finite-dimensional, we see that the $\mathfrak{m}$-torsion is nonzero precisely when the $\mathfrak{m}$-localization is nonzero. Thus, we may work with the localization at $\mathfrak{m}$ instead.

Now $R \pi_{H T *} \mathbb{F}_{\ell}$ (in fact, the $G\left(\mathbb{Q}_{p}\right)$-equivariant version $R\left(\pi_{H T} / G\left(\mathbb{Q}_{p}\right)\right)_{\text {ét }} \mathbb{F}_{\ell}$ ) is a sheaf of $\mathbb{T}_{S}$-modules, as the Hecke operators away from $p$ act trivially on $\mathscr{F} \ell_{G, \mu}$. We may thus form the localization $\left(R \pi_{H T *} \mathbb{F}_{\ell}\right)_{\mathfrak{m}}$. We claim that $\left(R \pi_{H T *} \mathbb{F}_{\ell}\right)_{\mathfrak{m}}$ is concentrated on the union $\mathscr{F} \ell_{\bar{G}, \mu}^{\geq d}$ of $\mathscr{F} \ell_{G, \mu}^{b^{\prime}}$ over all $b^{\prime}$ with $\left\langle 2 \rho, \nu_{b^{\prime}}\right\rangle \geq d$ (which is a closed subset of $\left.\mathscr{F} \ell_{G, \mu}\right)$.

Indeed, if $y \in \mathscr{F} \ell_{G, \mu}$ does not lie in $\mathscr{F} \ell_{G, \mu}^{\geq d}$, then it lies in $\mathscr{F} \ell_{G, \mu}^{b^{\prime}}$ for some $b^{\prime}$ with $\left\langle 2 \rho, \nu_{b^{\prime}}\right\rangle<d$. Now Theorem 4.4.4 computes the fibre of $R \pi_{H T *} \mathbb{F}_{\ell}$ at any geometric point above $y$ as $R \Gamma\left(\operatorname{Ig}^{b^{\prime}}, \mathbb{F}_{\ell}\right)$. We may pass to localizations at $\mathfrak{m}$ in this statement, and thus the assumption of the corollary shows that the localization of $\left(R \pi_{H T *} \mathbb{F}_{\ell}\right)_{\mathfrak{m}}$ at $y$ vanishes.

Next, we claim that for any affinoid étale $U \rightarrow \mathscr{F} \ell_{G, \mu}$ with formal model $\mathfrak{U}$, equivariant under $K_{p}$, with trivial action on $\mathfrak{U}_{s}$, the nearby cycles

$$
R \lambda_{U / K_{p} *}\left(\left.\left(R\left(\pi_{H T} / G\left(\mathbb{Q}_{p}\right)_{*} \mathbb{F}_{\ell}\right)_{\mathfrak{m}}\right)\right|_{U_{\bar{\eta}} / K_{p}}\right.
$$

are supported on a closed subset of $\mathfrak{U}_{\bar{s}}$ of dimension $\langle 2 \rho, \mu\rangle-d$. Indeed, the sheaf is supported on the closure in $\mathfrak{U}$ of the preimage $U^{\geq d} \subset U$ of $\mathscr{F} \ell_{\bar{G}, \mu}^{\geq d} \subset \mathscr{F} \ell_{G, \mu}$. But $U^{\geq d} \subset U$ is a closed subset of dimension $\leq\langle 2 \rho, \mu\rangle-d$, and then the same is true for its closure in $\mathfrak{U}$ : If $x \in \mathfrak{U}_{s}$ is a point whose closure is of dimension $e$, then the closure in $U$ of any lift $\tilde{x} \in U$ of $x$ will have at least dimension $e$ (as the specialization map is specializing).

Recall that $\left.R \lambda_{U / K_{p} *}\left(R\left(\pi_{H T} / G\left(\mathbb{Q}_{p}\right)\right)_{*} \mathbb{F}_{\ell}\right)\right|_{U_{\bar{\eta}} / K_{p}}[\langle 2 \rho, \mu\rangle]$ is perverse. It follows that the same is true for its localization

$$
\begin{aligned}
& \left(\left.R \lambda_{U / K_{p} *}\left(R\left(\pi_{H T} / G\left(\mathbb{Q}_{p}\right)\right)_{*} \mathbb{F}_{\ell}\right)\right|_{U_{\bar{\eta}} / K_{p}}[\langle 2 \rho, \mu\rangle]\right)_{\mathfrak{m}} \\
= & \left.R \lambda_{U / K_{p^{*}}}\left(\left(R\left(\pi_{H T} / G\left(\mathbb{Q}_{p}\right)\right)_{*} \mathbb{F}_{\ell}\right)_{\mathfrak{m}}\right)\right|_{U_{\bar{\eta}} / K_{p}}[\langle 2 \rho, \mu\rangle]
\end{aligned}
$$

at $\mathfrak{m}$. This sheaf is supported on a scheme of finite type of dimension $\langle 2 \rho, \mu\rangle-d$. It follows that the localization

$$
\left.\left(\left.R \lambda_{U / K_{p} *}\left(\left(R\left(\pi_{H T} / G\left(\mathbb{Q}_{p}\right)\right)_{*} \mathbb{F}_{\ell}\right)_{\mathfrak{m}}\right)\right|_{U_{\bar{\eta}} / K_{p}}\right)\right|_{\bar{x}}
$$

at any geometric point $\bar{x} \in \mathfrak{U}_{\bar{s}}$ whose closure is of dimension $\langle 2 \rho, \mu\rangle-d$ is concentrated in degree $d$.

Now pick $b$ as in the statement, and choose a rank 1 point $y \in \mathscr{F} \ell_{G, \mu}^{b}$ with $\operatorname{dim} \overline{\{y\}}=\langle 2 \rho, \mu\rangle-d$, and a geometric point $\bar{y}$ above $y$. One has an identification

$$
\left(R \pi_{H T *} \mathbb{F}_{\ell}\right)_{\mathfrak{m}, \bar{y}}=R \Gamma\left(\operatorname{Ig}^{b}, \mathbb{F}_{\ell}\right)_{\mathfrak{m}} .
$$

On the other hand, choose a cofinal system of affinoid étale neighborhoods $U_{i}=$ $\operatorname{Spa}\left(R_{i}, R_{i}^{\circ}\right) \rightarrow \mathscr{F}_{G, \mu}$ of $\bar{y}$ as in Proposition 6.1.3, with formal models $\mathfrak{U}_{i}$. Let $\bar{x}_{i} \in \mathfrak{U}_{i, s}$ be the specialization of $\bar{y}$, which is a geometric point of $\mathfrak{U}_{i, s}$. If $i$ is large enough, the dimension of the closure of $\bar{x}_{i}$ will be equal to $\langle 2 \rho, \mu\rangle-d$ : One needs 
to arrange that the image of $R_{i}^{\circ} \rightarrow \mathcal{O}_{K(\bar{y})} \rightarrow k(\bar{y})$, where $K(\bar{y})$ is the completed residue field at $\bar{y}$, with ring of integers $\mathcal{O}_{K(\bar{y})}$ and residue field $k(\bar{y})$, contains a transcendence basis. Also, choose compact open subgroups $K_{p, i} \subset G\left(\mathbb{Q}_{p}\right)$ that act on $U_{i}$ and trivially on $\mathfrak{U}_{i, s}$, such that the $K_{p, i}$ shrink to 1 .

In that situation, we know that for all large enough $i$

$$
\left.\left(\left.R \lambda_{U_{i} / K_{p_{i}} *}\left(\left(R\left(\pi_{H T} / G\left(\mathbb{Q}_{p}\right)\right)_{*} \mathbb{F}_{\ell}\right)_{\mathfrak{m}}\right)\right|_{U_{i, \bar{\eta}} / K_{p, i}}\right)\right|_{\bar{x}_{i}}
$$

is concentrated in degree $d$. Finally, we conclude by observing that

$$
\left(R \pi_{H T *} \mathbb{F}_{\ell}\right)_{\mathfrak{m}, \bar{y}}=\left.\underset{i}{\lim }\left(\left.R \lambda_{U_{i} / K_{p, i} *}\left(\left(R\left(\pi_{H T} / G\left(\mathbb{Q}_{p}\right)\right)_{*} \mathbb{F}_{\ell}\right)_{\mathfrak{m}}\right)\right|_{U_{i, \bar{\eta}} / K_{p, i}}\right)\right|_{\bar{x}_{i}} .
$$

6.2. A genericity assumption. In our main theorem, we impose a genericity assumption at some auxiliary prime. In this section, we briefly study this genericity condition.

Definition 6.2.1. Let $L$ be a $p$-adic field, and let

$$
\bar{\rho}: \operatorname{Gal}(\bar{L} / L) \rightarrow G L_{n}\left(\overline{\mathbb{F}}_{\ell}\right)
$$

be an unramified, continuous representation, with $\ell \neq p$. Then $\bar{\rho}$ is decomposed generic if the eigenvalues $\lambda_{1}, \ldots, \lambda_{n}$ of $\bar{\rho}$ (Frob) satisfy $\lambda_{a} / \lambda_{b} \notin\{1, q\}$ for all $a \neq b$, where Frob is an arithmetic Frobenius, and $q$ is the cardinality of the residue field of $L$.

We note that this condition actually only depends on the semisimplification of $\bar{\rho}$, but also implies that $\bar{\rho}$ is semisimple. In particular, if

$$
\rho: \operatorname{Gal}(\bar{L} / L) \rightarrow G L_{n}\left(\overline{\mathbb{Q}}_{\ell}\right)
$$

is a continuous representation, the condition that the reduction $\bar{\rho}$ be decomposed generic is unambiguous.

Lemma 6.2.2. Assume that

$$
\rho: \operatorname{Gal}(\bar{L} / L) \rightarrow G L_{n}\left(\overline{\mathbb{Q}}_{\ell}\right)
$$

is a continuous representation such that the reduction $\bar{\rho}$ is decomposed generic. Then $\rho$ decomposes as a sum $\rho=\bigoplus_{i=1}^{n} \chi_{i}$ of characters, and $\chi_{a} / \chi_{b}$ is not the cyclotomic character for any $a \neq b$.

In particular, the representation of $G L_{n}(L)$ corresponding to $\rho$ is a generic principal series representation.

Proof. We may conjugate $\rho$ into $G L_{n}\left(\mathcal{O}_{K}\right)$ for some finite extension $K \subset \overline{\mathbb{Q}}_{\ell}$. Writing $\bar{\rho}=\bigoplus_{i=1}^{n} \bar{\chi}_{i}$, we may further conjugate $\rho$ into the matrices in $G L_{n}\left(\mathcal{O}_{K}\right)$ which are diagonal modulo a uniformizer $\varpi$ of $\mathcal{O}_{K}$. Now we try to conjugate $\rho$ into the matrices which are diagonal modulo higher powers of $\varpi$. By standard calculations in deformation theory, the relevant obstruction groups are given by

$$
H^{1}\left(\operatorname{Gal}(\bar{L} / L), \bar{\chi}_{a} / \bar{\chi}_{b}\right)
$$

for $a \neq b$. But if

$$
\bar{\chi}_{\lambda}: \operatorname{Gal}(\bar{L} / L) \rightarrow \overline{\mathbb{F}}_{\ell}^{\times}
$$

denotes the unramified character sending Frob to $\lambda$, then it is well-known that

$$
H^{1}\left(\operatorname{Gal}(\bar{L} / L), \bar{\chi}_{\lambda}\right)=0
$$


if $\lambda \notin\{1, q\}$. By assumption, it follows that all relevant obstruction groups vanish.

The final statement follows because $\bar{\chi}_{a} / \bar{\chi}_{b}$ is not the cyclotomic character.

6.3. Conclusion. Finally, we can tie everything together and prove our main theorem.

Let us recall the relevant Shimura varieties. We fix a compact Shimura variety of PEL type, associated with PEL data $(B, *, V,(\cdot, \cdot))$ of type A satisfying one of the following assumptions. In both cases, $F=F^{+} \cdot \mathcal{K}$ is a $\mathrm{CM}$ field with totally real subfield $F^{+}$containing an imaginary-quadratic field $\mathcal{K}$.

Case 1. Assume that $B$ is a central division algebra over $F$, and $V \cong B$ is a simple $B$-module.

Case 2. Assume that $B=F, F^{+} \neq \mathbb{Q}$, the corresponding group $G$ is quasi-split at all finite places, and if a rational prime $q$ is ramified in $F$, then $F / F^{+}$is split at all places above $q$.

In both cases, let $\mathrm{Spl}_{F / F^{+}}$denote the set of rational primes $q$ such that every place of $F^{+}$above $q$ splits in $F$. Moreover, fix a finite set $S$ of primes such that $F$ and $G$ are unramified outside $S$, and pick a sufficiently small compact open subgroup $K=K_{S} K^{S} \subset G\left(\mathbb{A}_{f}\right)=G\left(\mathbb{A}_{S}\right) \times G\left(\mathbb{A}_{f}^{S}\right)$ such that $K^{S}$ is a product of hyperspecial maximal compact open subgroups $K_{q} \subset G\left(\mathbb{Q}_{q}\right)$. In Case 2, we assume that $S \subset \mathrm{Spl}_{F / F^{+}}$. Finally, take some rational prime $\ell$. We will consider the following abstract Hecke algebra

$$
\mathbb{T}^{S}=\bigotimes_{q \in \operatorname{Spl}_{\mathcal{K} / \mathbb{Q}} \backslash(S \cup\{\ell\})} \mathbb{Z}\left[G\left(\mathbb{Q}_{q}\right) / / K_{q}\right] .
$$

Theorem 6.3.1. Let $\mathfrak{m} \subset \mathbb{T}^{S}$ be a maximal ideal such that

$$
H^{i}\left(S_{K}, \mathbb{F}_{\ell}\right)_{\mathfrak{m}} \neq 0
$$

for some $i \in \mathbb{Z}$.

(1) There is a (unique) semisimple continuous Galois representation

$$
\rho_{\mathfrak{m}}: \operatorname{Gal}(\bar{F} / F) \rightarrow G L_{n}\left(\overline{\mathbb{F}}_{\ell}\right)
$$

unramified outside the places above $S \cup\{\ell\}$, such that for all finite places $v$ lying above a prime $q \in \mathrm{Spl}_{\mathcal{K} / \mathbb{Q}} \backslash(S \cup\{\ell\})$, the characteristic polynomial of $\rho_{\mathfrak{m}}\left(\right.$ Frob $\left._{v}\right)$ is given by the image of

$$
X^{n}-T_{1, v} X^{n-1} \pm \ldots+(-1)^{i} q_{v}^{i(i-1) / 2} T_{i, v} X^{n-i}+\ldots+(-1)^{n} q_{v}^{n(n-1) / 2} T_{n, v}
$$

under a fixed embedding $\mathbb{T}^{S} / \mathfrak{m} \hookrightarrow \overline{\mathbb{F}}_{\ell}$, where $q_{v}$ is the cardinality of the residue field at $v$, and

$$
T_{i, v} \in \mathbb{Z}\left[G\left(\mathbb{Q}_{q}\right) / / K_{q}\right]
$$

is the characteristic function of

$$
G L_{n}\left(\mathcal{O}_{F_{v}}\right) \operatorname{diag}(\underbrace{\varpi_{v}, \ldots, \varpi_{v}}_{i}, 1, \ldots, 1) G L_{n}\left(\mathcal{O}_{F_{v}}\right) \times \prod_{w \neq v} G L_{n}\left(\mathcal{O}_{F_{w}}\right) \times \mathbb{Z}_{p}^{\times}
$$

inside

$$
G\left(\mathbb{Q}_{q}\right)=\prod_{w} G L_{n}\left(F_{w}\right) \times \mathbb{Q}_{p}^{\times},
$$

where $w$ runs over all places of $F$ lying over the same place of $\mathcal{K}$ as $v$. 
(2) Assume that there is some rational prime $p \in \operatorname{Spl}_{\mathcal{K} / \mathbb{Q}} \backslash(S \cup\{\ell\})$, split as $p=$ $u u^{c}$ in $\mathcal{K}$, and a prime $\mathfrak{p} \mid p$ of $E$ such that the following condition involving the primes $\mathfrak{p}_{i} \mid u$ of $F, i=1, \ldots, m$, and the sets $S_{i}$ from Theorem 5.5.4 holds true. For any $i$, there is at most one $\tau \in S_{i}$ such that $p_{\tau} q_{\tau} \neq 0$; if there is such a $\tau \in S_{i}$, then $\rho_{\mathfrak{m}}$ is decomposed generic at $\mathfrak{p}_{i}$. Then

$$
H^{i}\left(S_{K}, \mathbb{F}_{\ell}\right)_{\mathfrak{m}} \neq 0
$$

only for $i=\operatorname{dim} S_{K}$.

Before giving the proof, let us explain in two examples how the condition (2) can be ensured, thus connecting it with the conditions stated in the introduction.

Remark 6.3.2. Assume that there is a prime $p$ which is completely decomposed in $F$ and such that $\rho_{\mathfrak{m}}$ is unramified and decomposed generic at all places above $p$. Using Chebotarev, there are then many such $p$, and we can assume that $p \notin S \cup\{\ell\}$. In that case, all sets $S_{i}$ in (2) have just one element, and we see that the desired condition is satisfied.

Remark 6.3.3. Assume that the signature of $G$ is $(0, n)$ at all except for one infinite place. Moreover, assume that there is some finite prime $v$ of $F$ such that $\rho_{\mathfrak{m}}$ is unramified and decomposed generic at $v$. By Chebotarev, there are then many such $v$ which are moreover decomposed over the rational prime $p$ of $\mathbb{Q}$, with $p \notin S \cup\{\ell\} 33$ In particular, $p$ needs to be split in $\mathcal{K}$. There is just one $\tau$ for which $p_{\tau} q_{\tau} \neq 0$, and by choosing the prime $\mathfrak{p}$ of the reflex field correctly, one can arrange that this $\tau$ appears in $S_{i}$ for $\mathfrak{p}_{i}=v$. We see that condition (2) applies.

Proof. We write out the argument in the more involved Case 2.

For part (1), pick any $p \in \operatorname{Spl}_{\mathcal{K} / \mathbb{Q}} \backslash(S \cup\{\ell\})$. Then $K=K_{p} K^{p}$ is decomposed. There is a Hochschild-Serre spectral sequence relating

$$
H^{i}\left(\mathcal{S}_{K^{p}}, \mathbb{F}_{\ell}\right)
$$

and $H^{i}\left(S_{K}, \mathbb{F}_{\ell}\right) 34$ In particular, it follows that if $i$ is minimal with $H^{i}\left(S_{K}, \mathbb{F}_{\ell}\right)_{\mathfrak{m}} \neq$ 0 , then

$$
H^{i}\left(\mathcal{S}_{K^{p}}, \mathbb{F}_{\ell}\right)_{\mathfrak{m}} \neq 0 .
$$

Thus, there is some $b \in B\left(G, \mu^{-1}\right)$ such that

$$
H^{i}\left(\operatorname{Ig}^{b}, \mathbb{F}_{\ell}\right)_{\mathfrak{m}} \neq 0
$$

for some $i \in \mathbb{Z}$; otherwise we would have

$$
\left(R \pi_{H T *} \mathbb{F}_{\ell}\right)_{\mathfrak{m}}=0,
$$

and hence

$$
R \Gamma\left(\mathcal{S}_{K^{p}}, \mathbb{F}_{\ell}\right)_{\mathfrak{m}}=0
$$

by the Leray spectral sequence for $\pi_{H T}: \mathcal{S}_{K^{p}} \rightarrow \mathscr{F} \ell_{G, \mu}$. Now pick some $b \in$ $B\left(G, \mu^{-1}\right)$ with $d=\left\langle 2 \rho, \nu_{b}\right\rangle$ minimal such that for some $i \in \mathbb{Z}$

$$
H^{i}\left(\operatorname{Ig}^{b}, \mathbb{F}_{\ell}\right)_{\mathfrak{m}} \neq 0 \text {. }
$$

\footnotetext{
${ }^{33}$ In Chebotarev's theorem, only places with residue field $\mathbb{F}_{p}$ contribute to the Dirichlet density.

${ }^{34}$ Here and in the following, all cohomology groups are étale cohomology groups after base change to an algebraically closed field.
} 
In that case, this group is nonzero exactly for $i=d$ by Corollary 6.1.4 Taking invariants under a pro-p-compact open subgroup of $J_{b}\left(\mathbb{Q}_{p}\right)$ (which is an exact operation), this implies that

$$
H^{i}\left(\mathscr{I}_{\text {Mant }, m}^{b}, \mathbb{F}_{\ell}\right)_{\mathfrak{m}}
$$

is nonzero at most for $i=d$; if $m$ is large enough, it is nonzero if $i=d$. It follows

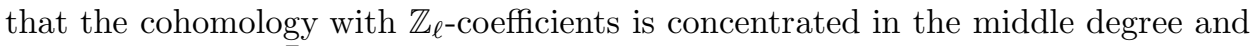
flat, and thus the $\overline{\mathbb{Q}}_{\ell}$-cohomology

$$
H^{i}\left(\mathscr{I}_{\mathrm{Mant}, m}^{b}, \mathbb{Z}_{\ell}\right)_{\mathfrak{m}} \otimes \overline{\mathbb{Q}}_{\ell}
$$

is nonzero for $i=d$. By Poincaré duality (and applying the same discussion with the "dual" set of Hecke eigenvalues), the same holds true for compactly supported cohomology. We have a decomposition

$$
\left[H_{c}\left(\mathscr{I}_{\text {Mant }}^{b}, \overline{\mathbb{Q}}_{\ell}\right)\right]^{S u r}=\left[H_{c}\left(\mathscr{I}_{\text {Mant }}^{b}, \overline{\mathbb{Q}}_{\ell}\right)\right]_{\mathfrak{m}}^{\text {Sur }}+\left[H_{c}\left(\mathscr{I}_{\text {Mant }}^{b}, \overline{\mathbb{Q}}_{\ell}\right)\right]^{S u r, \mathfrak{m}}
$$

according to systems of Hecke eigenvalues lifting $\mathfrak{m}$, or a different set of Hecke eigenvalues modulo $\ell$, and by concentration in one degree, the first summand is nonzero in the Grothendieck group, and its base change $B C^{p}$ is still nonzero. It follows that there is some $\Pi^{\vec{n}}$ as in Lemma 5.5.1 whose Hecke eigenvalues lift $\mathfrak{m}$. Then Theorem 5.5.3 implies that there is a Galois representation $r_{\Pi^{\vec{n}}, \ell}$, whose reduction is the desired Galois representation $\rho_{\mathfrak{m}}$.

Now, we deal with part (2). We choose $p$ and $\mathfrak{p}$ as guaranteed in the statement. It is enough to prove that $H^{i}\left(S_{K}, \mathbb{F}_{\ell}\right)_{\mathfrak{m}}$ is nonzero only for $i \geq \operatorname{dim} S_{K}$; the other bound follows by Poincaré duality (and the result for the "dual" ideal, which satisfies the same hypothesis). Now a Hochschild-Serre spectral sequence shows that it is enough to prove that

$$
H^{i}\left(\mathcal{S}_{K^{p}}, \mathbb{F}_{\ell}\right)_{\mathfrak{m}}=0
$$

for $i<\operatorname{dim} S_{K}$. As above, we take some $b \in B\left(G, \mu^{-1}\right)$ with $d=\left\langle 2 \rho, \nu_{b}\right\rangle$ minimal such that

$$
H^{i}\left(\operatorname{Ig}^{b}, \mathbb{F}_{\ell}\right)_{\mathfrak{m}} \neq 0
$$

for some $i \in \mathbb{Z}$. We get concentration in middle degree in this case, and hence the argument above shows that there is some Galois representation $r$ lifting $\rho_{\mathfrak{m}}$ with

$$
B C^{p}\left(\left[H_{c}\left(\mathscr{I}_{\text {Mant }}^{b}, \overline{\mathbb{Q}} \ell\right)^{S \text { ur }}\right)_{r} \neq 0 .\right.
$$

But by Lemma 6.2.2 and the assumptions on $p, \mathfrak{p}$ and $\rho_{\mathfrak{m}}$, the hypothesis of Theorem 5.5.4 are satisfied. Thus, if $b$ is not $\mu$-ordinary, we arrive at a contradiction. It follows that $b$ is $\mu$-ordinary.

In that case, $\langle 2 \rho, \mu\rangle=\left\langle 2 \rho, \nu_{b}\right\rangle=\operatorname{dim} S_{K}$, so Corollary 6.1.4 shows that

$$
H^{i}\left(\operatorname{Ig}^{b}, \mathbb{F}_{\ell}\right)_{\mathfrak{m}}
$$

vanishes for $i<\operatorname{dim} S_{K}$, for all $b \in B\left(G, \mu^{-1}\right)$. Thus, $\left(R^{i} \pi_{H T *} \mathbb{F}_{\ell}\right)_{\mathfrak{m}}$ vanishes for $i<\operatorname{dim} S_{K}$, and the result follows by applying the Leray spectral sequence for $\pi_{H T}: \mathcal{S}_{K^{p}} \rightarrow \mathscr{F} \ell_{G, \mu}$. 


\section{REFERENCES}

[Art88a] James Arthur, The invariant trace formula. I. Local theory, J. Amer. Math. Soc. 1 (1988), no. 2, 323-383.

[Art88b] - The invariant trace formula. II. Global theory, J. Amer. Math. Soc. 1 (1988), no. $3,501-554$.

[Art89] , The $L^{2}$-Lefschetz numbers of Hecke operators, Invent. Math. 97 (1989), no. 2, $257-290$.

[Art13] - The endoscopic classification of representations, American Mathematical Society Colloquium Publications, vol. 61, American Mathematical Society, Providence, RI, 2013, Orthogonal and symplectic groups.

[Bad07] Alexandru Ioan Badulescu, Jacquet-Langlands et unitarisabilité, J. Inst. Math. Jussieu 6 (2007), no. 3, 349-379.

[BBM82] Pierre Berthelot, Lawrence Breen, and William Messing, Théorie de Dieudonné cristalline. II, Lecture Notes in Mathematics, vol. 930, Springer-Verlag, Berlin, 1982.

[Ber80] Pierre Berthelot, Théorie de Dieudonné sur un anneau de valuation parfait, Ann. Sci. École Norm. Sup. (4) 13 (1980), no. 2, 225-268.

[Bla94] Don Blasius, A p-adic property of Hodge classes on abelian varieties, Motives (Seattle, WA, 1991), Proc. Sympos. Pure Math., vol. 55, Amer. Math. Soc., Providence, RI, 1994, pp. 293-308.

[Boy15] Pascal Boyer, Sur la torsion dans la cohomologie des varits de Shimura de Kottwitz-Harris-Taylor, https://www.math.univ-paris13.fr/ boyer/recherche/torsionlocalise.pdf.

[BS15a] Bhargav Bhatt and Peter Scholze, The proétale topology for schemes, Astérisque 369 (2015).

[BS15b] - Projectivity of the Witt vector affine Grassmannian, arXiv:1507.06490.

[BW80] Armand Borel and Nolan R. Wallach, Continuous cohomology, discrete subgroups, and representations of reductive groups, Annals of Mathematics Studies, vol. 94, Princeton University Press, Princeton, N.J.; University of Tokyo Press, Tokyo, 1980.

[Car12] Ana Caraiani, Local-global compatibility and the action of monodromy on nearby cycles, Duke Math. J. 161 (2012), no. 12, 2311-2413.

[CG] Frank Calegari and David Geraghty, Modularity lifting beyond the taylor-wiles method, https://www2.bc.edu/david-geraghty/files/merge.pdf.

[CH13] Gaëtan Chenevier and Michael Harris, Construction of automorphic Galois representations, II, Camb. J. Math. 1 (2013), no. 1, 53-73.

[Del70] Pierre Deligne, Équations différentielles à points singuliers réguliers, Lecture Notes in Mathematics, Vol. 163, Springer-Verlag, Berlin-New York, 1970.

[Del71] _ Travaux de Shimura, Séminaire Bourbaki, 23ème année (1970/71), Exp. No. 389, Springer, Berlin, 1971, pp. 123-165. Lecture Notes in Math., Vol. 244.

[Del77] P. Deligne, Cohomologie étale, Lecture Notes in Mathematics, Vol. 569, Springer-Verlag, Berlin-New York, 1977, Séminaire de Géométrie Algébrique du Bois-Marie SGA 4 1/2, Avec la collaboration de J. F. Boutot, A. Grothendieck, L. Illusie et J. L. Verdier.

[Del79] Pierre Deligne, Variétés de Shimura: interprétation modulaire, et techniques de construction de modèles canoniques, Automorphic forms, representations and $L$-functions (Proc. Sympos. Pure Math., Oregon State Univ., Corvallis, Ore., 1977), Part 2, Proc. Sympos. Pure Math., XXXIII, Amer. Math. Soc., Providence, R.I., 1979, pp. 247-289.

[Del82] - Hodge cycles on abelian varieties, Hodge cycles, motives, and Shimura varieties, Lecture Notes in Mathematics, vol. 900, Springer-Verlag, Berlin-New York, 1982, pp. ii +414 .

[EG15] Matthew Emerton and Toby Gee, p-adic Hodge-theoretic properties of étale cohomology with $\bmod p$ coefficients, and the cohomology of Shimura varieties, Algebra Number Theory 9 (2015), no. 5, 1035-1088.

[Far] Laurent Fargues, Geométrisation de la correspondance de Langlands, in preparation.

[Far15a] — G-torseurs en théorie de Hodge p-adique, http://webusers.imjprg.fr/ laurent.fargues/Gtorseurs.pdf.

[Far15b] Luelques résultats et conjectures concernant la courbe, Astérisque 369 (2015). 
[FF14] L. Fargues and J. M. Fontaine, Vector bundles on curves and p-adic Hodge theory, Automorphic Forms and Galois representations, Volume I, London Math. Soc. Lec. Note., vol. Ser. 414, Cambrigde Univ. Press, 2014.

[Fra98] Jens Franke, Harmonic analysis in weighted $L_{2}$-spaces, Ann. Sci. École Norm. Sup. (4) 31 (1998), no. 2, 181-279.

[Gol14] Wushi Goldring, Galois representations associated to holomorphic limits of discrete series, Compos. Math. 150 (2014), no. 2, 191-228.

[Ham] P. Hamacher, The geometry of Newton strata in the reduction modulo $p$ of Shimura varieties of PEL type, to appear in Duke Math. J.

[HT01] Michael Harris and Richard Taylor, The geometry and cohomology of some simple Shimura varieties, Annals of Mathematics Studies, vol. 151, Princeton University Press, Princeton, NJ, 2001, With an appendix by Vladimir G. Berkovich.

[Hub96] Roland Huber, Étale cohomology of rigid analytic varieties and adic spaces, Aspects of Mathematics, E30, Friedr. Vieweg \& Sohn, Braunschweig, 1996.

[Ill94] Luc Illusie, Autour du théorème de monodromie locale, Astérisque (1994), no. 223, 9-57, Périodes $p$-adiques (Bures-sur-Yvette, 1988).

[JS81] H. Jacquet and J. A. Shalika, On Euler products and the classification of automorphic forms. II, Amer. J. Math. 103 (1981), no. 4, 777-815.

[Kat79] Nicholas M. Katz, Slope filtration of F-crystals, Journées de Géométrie Algébrique de Rennes (Rennes, 1978), Vol. I, Astérisque, vol. 63, Soc. Math. France, Paris, 1979, pp. 113-163.

[Kis] M. Kisin, Mod p points on Shimura varieties of abelian type, preprint.

[Kis10] Mark Kisin, Integral models for Shimura varieties of abelian type, J. Amer. Math. Soc. 23 (2010), no. 4, 967-1012.

[KL15] K. S. Kedlaya and R. Liu, Relative p-adic Hodge theory, I: Foundations, Astérisque 371 (2015).

[Kot84] Robert E. Kottwitz, Stable trace formula: cuspidal tempered terms, Duke Math. J. 51 (1984), no. 3, 611-650.

[Kot85] — Isocrystals with additional structure, Compositio Math. 56 (1985), no. 2, 201220.

[Kot90] - Shimura varieties and $\lambda$-adic representations, Automorphic forms, Shimura varieties, and $L$-functions, Vol. I (Ann Arbor, MI, 1988), Perspect. Math., vol. 10, Academic Press, Boston, MA, 1990, pp. 161-209.

[Kot92a] _ On the $\lambda$-adic representations associated to some simple Shimura varieties, Invent. Math. 108 (1992), no. 3, 653-665.

[Kot92b] - Points on some Shimura varieties over finite fields, J. Amer. Math. Soc. 5 (1992), no. 2, 373-444.

[Kud94] Stephen S. Kudla, The local Langlands correspondence: the non-Archimedean case, Motives (Seattle, WA, 1991), Proc. Sympos. Pure Math., vol. 55, Amer. Math. Soc., Providence, RI, 1994, pp. 365-391.

[Lab91] J.-P. Labesse, Pseudo-coefficients très cuspidaux et K-théorie, Math. Ann. 291 (1991), no. 4, 607-616.

[Lab99] Jean-Pierre Labesse, Cohomologie, stabilisation et changement de base, Astérisque (1999), no. 257, vi+161, Appendix A by Laurent Clozel and Labesse, and Appendix B by Lawrence Breen.

[Lan79] R. P. Langlands, Stable conjugacy: definitions and lemmas, Canad. J. Math. 31 (1979), no. $4,700-725$

[Lan11] Kai-Wen Lan, Elevators for degenerations of PEL structures, Math. Res. Lett. 18 (2011), no. 5, 889-907.

[Lau13] Eike Lau, Smoothness of the truncated display functor, J. Amer. Math. Soc. 26 (2013), no. $1,129-165$

[LS87] R. P. Langlands and D. Shelstad, On the definition of transfer factors, Math. Ann. 278 (1987), no. 1-4, 219-271.

[LS12] Kai-Wen Lan and Junecue Suh, Vanishing theorems for torsion automorphic sheaves on compact PEL-type Shimura varieties, Duke Math. J. 161 (2012), no. 6, 1113-1170.

[Man05] Elena Mantovan, On the cohomology of certain PEL-type Shimura varieties, Duke Math. J. 129 (2005), no. 3, 573-610. 
[Mat67] Yozô Matsushima, A formula for the Betti numbers of compact locally symmetric Riemannian manifolds, J. Differential Geometry 1 (1967), 99-109.

[Mes72] William Messing, The crystals associated to Barsotti-Tate groups: with applications to abelian schemes, Lecture Notes in Mathematics, Vol. 264, Springer-Verlag, Berlin-New York, 1972.

[Mil90] J. S. Milne, Canonical models of (mixed) Shimura varieties and automorphic vector bundles, Automorphic forms, Shimura varieties, and $L$-functions, Vol. I (Ann Arbor, MI, 1988), Perspect. Math., vol. 10, Academic Press, Boston, MA, 1990, pp. 283-414.

[MW89] C. Mœglin and J.-L. Waldspurger, Le spectre résiduel de $\operatorname{GL}(n)$, Ann. Sci. École Norm. Sup. (4) 22 (1989), no. 4, 605-674.

[Ngô10] Bao Châu Ngô, Le lemme fondamental pour les algèbres de Lie, Publ. Math. Inst. Hautes Études Sci. (2010), no. 111, 1-169.

[NP01] B. C. Ngô and P. Polo, Résolutions de Demazure affines et formule de Casselman-Shalika géométrique, J. Algebraic Geom. 10 (2001), no. 3, 515-547.

[OZ02] Frans Oort and Thomas Zink, Families of p-divisible groups with constant Newton polygon, Doc. Math. 7 (2002), 183-201 (electronic).

[Rap15] Michael Rapoport, Accessible and weakly accessible period domains, appendix to http://www.math.uni-bonn.de/people/scholze/pAdicCohomLT.pdf.

[RR96] M. Rapoport and M. Richartz, On the classification and specialization of F-isocrystals with additional structure, Compositio Math. 103 (1996), no. 2, 153-181.

[RZ96] M. Rapoport and Th. Zink, Period spaces for p-divisible groups, Annals of Mathematics Studies, vol. 141, Princeton University Press, Princeton, NJ, 1996.

[Sch11] Peter Scholze, Letter to Jean-Francois Dat, April 28, 2011.

[Sch12a] — Perfectoid spaces, Publ. Math. Inst. Hautes Études Sci. 116 (2012), 245-313.

[Sch12b] _ Perfectoid Spaces: A survey, Current Developments in Mathematics (2012).

[Sch13a] _ The Langlands-Kottwitz method and deformation spaces of p-divisible groups, J. Amer. Math. Soc. 26 (2013), no. 1, 227-259.

[Sch13b] _ The local Langlands correspondence for $G L_{n}$ over p-adic fields, Invent. Math. 192 (2013), no. 3, 663-715.

[Sch13c] _ p-adic Hodge theory for rigid-analytic varieties, Forum Math. Pi 1 (2013), e1, 77 .

[Sch15a] _ On the p-adic cohomology of the Lubin-Tate tower, http://www.math.unibonn.de/people/scholze/pAdicCohomLT.pdf.

[Sch15b] — On torsion in the cohomology of locally symmetric varieties, Annals of Mathematics (2015).

[SGA73] Groupes de monodromie en géométrie algébrique. II, Lecture Notes in Mathematics, Vol. 340, Springer-Verlag, Berlin-New York, 1973, Séminaire de Géométrie Algébrique du Bois-Marie 1967-1969 (SGA 7 II), Dirigé par P. Deligne et N. Katz.

[Shi09] Sug Woo Shin, Counting points on Igusa varieties, Duke Math. J. 146 (2009), no. 3, $509-568$.

[Shi10] , A stable trace formula for Igusa varieties, J. Inst. Math. Jussieu 9 (2010), no. 4, 847-895.

[Shi11] Galois representations arising from some compact Shimura varieties, Ann. of Math. (2) 173 (2011), no. 3, 1645-1741.

[Shi12] _ On the cohomology of Rapoport-Zink spaces of EL-type, Amer. J. Math. 134 (2012), no. 2, 407-452.

[Shi15] - Supercuspidal part of the mod l cohomology of $G U(1, n-1)$-Shimura varieties, J. Reine Angew. Math. 705 (2015), 1-21.

[SS13] Peter Scholze and Sug Woo Shin, On the cohomology of compact unitary group Shimura varieties at ramified split places, J. Amer. Math. Soc. 26 (2013), no. 1, 261-294.

[SW13] Peter Scholze and Jared Weinstein, Moduli of p-divisible groups, Camb. J. Math. 1 (2013), no. $2,145-237$.

[Tho14] Jack A. Thorne, Raising the level for $\mathrm{GL}_{n}$, Forum Math. Sigma 2 (2014), e16, 35.

[Wal97] J.-L. Waldspurger, Le lemme fondamental implique le transfert, Compositio Math. 105 (1997), no. 2, 153-236.

[Wed99] Torsten Wedhorn, Ordinariness in good reductions of Shimura varieties of PEL-type, Ann. Sci. École Norm. Sup. (4) 32 (1999), no. 5, 575-618. 
[Wei14] Jared Weinstein, Peter Scholze's lectures on p-adic geometry, Available online at http://math.berkeley.edu/ jared/Math274/index.html (2014).

E-mail address: caraiani@princeton.edu

Department of Mathematics, Princeton University, Fine Hall, Washington Rd., PrinceTON, NJ 08544, USA

E-mail address: scholze@math.uni-bonn.de

Mathematisches Institut der Universität Bonn, Endenicher Allee 60, 53115 Bonn, GERMANY 